Gesine Fuchs

Silke Bothfeld

Andrea Leitner

Sophie Rouault

\title{
Gleichstellungspolitik öffentlicher Arbeitgeber
}


Gesine Fuchs • Silke Bothfeld • Andrea Leitner - Sophie Rouault

Gleichstellungspolitik öffentlicher Arbeitgeber 


\section{Gesine Fuchs \\ Silke Bothfeld \\ Andrea Leitner \\ Sophie Rouault}

\section{Gleichstellungspolitik \\ öffentlicher Arbeitgeber}

Betriebliche Gleichstellung in den Bundesverwaltungen Deutschlands, Österreichs und der Schweiz

Verlag Barbara Budrich

Opladen • Berlin • Toronto 2016 
Bibliografische Information der Deutschen Nationalbibliothek

Die Deutsche Nationalbibliothek verzeichnet diese Publikation in der Deutschen

Nationalbibliografie; detaillierte bibliografische Daten sind im Internet über

http://dnb.d-nb.de abrufbar.

(C) 2018 Dieses Werk ist beim Verlag Barbara Budrich erschienen und steht unter der Creative Commons Lizenz Attribution-ShareAlike 4.0 International (CC BY-SA 4.0): https://creativecommons.org/licenses/by-sa/4.0/

Diese Lizenz erlaubt die Verbreitung, Speicherung, Vervielfältigung und Bearbeitung bei Verwendung der gleichen CC-BY-SA 4.0-Lizenz und unter Angabe der UrheberInnen, Rechte, Änderungen und verwendeten Lizenz.

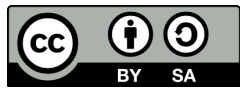

Dieses Buch steht im Open-Access-Bereich der Verlagsseite zum kostenlosen Download bereit (https://doi.org/10.3224/84740191).

Eine kostenpflichtige Druckversion (Print on Demand) kann über den Verlag bezogen werden. Die Seitenzahlen in der Druck- und Onlineversion sind identisch.

$$
\begin{array}{ll}
\text { ISBN } & 978-3-8474-0191-9 \text { (Paperback) } \\
\text { eISBN } & 978-3-8474-0414-9 \text { (eBook) } \\
\text { DOI } & 10.3224 / 84740191
\end{array}
$$

Umschlaggestaltung: Bettina Lehfeldt, Kleinmachnow - www.lehfeldtgraphic.de Typographisches Lektorat: Anja Borkam, Jena 


\section{Inhaltsverzeichnis}

Verzeichnis der Abbildungen .............................................................. 10

Verzeichnis der Tabellen .................................................................... 11

Verzeichnis der Tafeln ................................................................... 11

1 Betriebliche Gleichstellungspolitik des öffentlichen Dienstes als Forschungsfeld: Einleitung …....................................... 13

Silke Bothfeld, Gesine Fuchs, Andrea Leitner, Sophie Rouault

2 Konzeptionelle Grundlagen und Untersuchungsdesign ........... 19

Silke Bothfeld, Gesine Fuchs, Andrea Leitner, Sophie Rouault

2.1 Betriebliche Gleichstellungspolitik in der Bundesverwaltung als

Teil der nationalen Geschlechterregimes

2.2 Die Bundesverwaltung als vergeschlechtlichte Organisation und Teil eines Geschlechterregimes .................................................. 22

2.3 Das Policy-Regime der beruflichen Gleichstellung ...................... 25

2.4 Das Steuerungsregime der Gleichstellung in der Bundesverwaltung .............................................................. 28

2.5 Untersuchungsdesign und empirisches Vorgehen ........................ 32

3 Die Bundesverwaltungen als Beschäftigungsfeld .................... 37

Silke Bothfeld, Gesine Fuchs, Andrea Leitner, Sophie Rouault

3.1 Grundprinzipien und Entwicklungslinien des öffentlichen Dienstes ........................................................................ 38

3.2 Rechtsgrundlagen der Gleichstellung im Bundesdienst ................ 40

3.3 Öffentlich-rechtliche Dienstverhältnisse ..................................... 42

3.4 Beschäftigte im Bundesdienst ..................................................... 45

3.5 Entlohnung und Gender Pay Gap .................................................. 48

3.6 Frauen und Männer in den Hierarchien des Bundesdienstes ......... 51

3.7 Zwischenfazit: Gleichstellungspolitik im Spannungsfeld zwischen gleichstellungspolitischen Anforderungen und traditionellen Strukturen 
4 Betriebliche Gleichstellung in der schweizerischen

Bundesverwaltung

Gesine Fuchs

4.1 Von der Rückständigkeit zu ungleichzeitigen Fortschritten:

Gleichstellungspolitische Kontextbedingungen ........................... 55

4.1.1 Veränderungen im schweizerischen Geschlechterregime .............. 55

4.1.2 Rechtliche Grundlagen beruflicher Gleichstellungspolitik ............ 58

4.2 Frauen und Männer beim Bundespersonal: Langsame

Feminisierung und starke Segregation ....................................... 62

4.3 Institutionelle Politik in der schweizerischen Bundesverwaltung ... 66

4.3.1 Personalrechtliche Grundlagen betrieblicher Gleichstellung in der

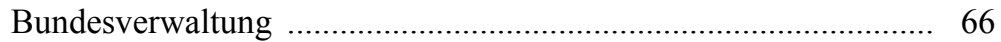

4.3.2 Verantwortliche für die betriebliche Gleichstellung: Heterogene Institutionalisierung .............................................................. 71

4.3.3 Gleichstellungspläne: Auf dem Weg zum Gleichstellungscontrolling?

4.3.4 Zwischenfazit: Institutionalisierung lediglich als Teil der Personalpolitik ...................................................................... 80

4.4 Substanzielle Politik in der schweizerischen Bundesverwaltung ... 84

4.4.1 Programme: Umfassend und à la carte ....................................... 84

4.4.2 Maßnahmen zur Vereinbarkeit von Familie und Beruf: Langsamer Abschied von der Anwesenheitskultur ....................................... 86

4.4.3 Maßnahmen zur Karriereförderung von Frauen: Geringe Verbindlichkeit ................................................................ 90

4.5 Fazit: Große institutionelle Handlungsspielräume, schwache Koordination .............................................................................. 94

5 Berufliche Gleichstellung im deutschen Bundesdienst: Vorbild für die widerständige Privatwirtschaft?

Sophie Rouault

5.1 Kontextbedingungen eines gespaltenen Regimes zur beruflichen Gleichstellung

5.1.1 Berufliche Gleichstellung in der Privatwirtschaft: Die betriebliche Freiheit, zu handeln 
5.1.2 Berufliche Gleichstellung im Bundesdienst: Inkrementelle Institutionalisierung eines Politikfelds ......................................... 103

5.1.3 Gespaltenes Policy-Regime zur beruflichen Gleichstellung ........... 105

5.2 Neuere Entwicklungen beim deutschen Bundespersonal: Immer mehr Frauen (in Teilzeit) .......................................................... 106

5.3 Institutionelle Gleichstellungspolitik in der deutschen Bundesverwaltung: Eine inkrementelle Dynamik ........................ 109

5.3.1 Bundesfamilienministerium - schwache Koordinierungsinstanz ... 110

5.3.2 Gleichstellungsbeauftragte in der Bundesverwaltung: Ein ausdifferenziertes Amt

5.3.3 „Gleichstellungscontrolling“ durch Gleichstellungspläne: Eine Führungsfrage .....

5.4 Substanzielle Gleichstellungspolitik in der deutschen Bundesverwaltung: Auf der Suche nach dem kleinsten gemeinsamen Nenner

5.4.1 Breit gefächerte Instrumente der Vereinbarkeit von Beruf und Familie im Bundesdienst

5.4.2 Vereinbarkeit von Beruf und Familie oder Reduktion von Gleichstellungspolitik auf konsensuale Maßnahmen

5.4.3 Mehr Frauen in Führungsposition: Unzureichende Quotenregelungen

5.5 Fazit zur deutschen Bundesverwaltung: Ein normativ kohärentes, aber technisch inkonsistentes Steuerungsregime zur beruflichen Gleichstellung

6 Gleichstellungspolitik im österreichischen Bundesdienst Andrea Leitner

6.1 Kontextbedingungen im Spannungsfeld von Familien- und

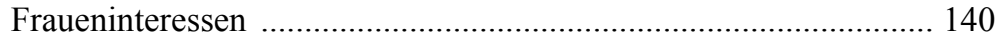

6.2 Frauen im österreichischen Bundesdienst ..................................... 144

6.2.1 Feminisierung mit Ausnahme von Militär- und Exekutivdienst ..... 145

6.2.2 Auflösungs- und Beharrungstendenzen der vertikalen Segregation

6.2.3 Unterschiede zwischen Ressorts

6.2.4 Zwischenfazit: Segregierte Feminisierung 
6.3 Institutionelle Politik im österreichischen Bundesdienst ............... 160

6.3.1 Bundes-Gleichbehandlungsgesetz als Rahmen der

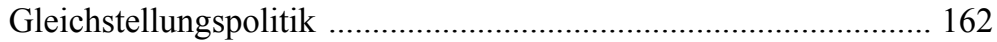

6.3.2 Frauenförderpläne als ,soft laws“ “.............................................. 162

6.3.3 Gleichbehandlungsbeauftragte als individuelle AkteurInnen ......... 165

6.3.4 Zwischenfazit: Trotz starker Institutionalisierung wenig

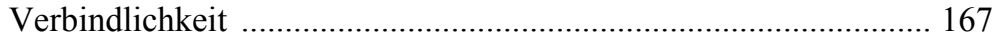

6.4 Substanzielle Politik im österreichischen Bundesdienst ................ 169

6.4.1 Maßnahmenvielfalt zur Vereinbarkeit von Familie und Beruf ....... 170

6.4.2 Versuche zur Vermeidung von Karriererisiken durch

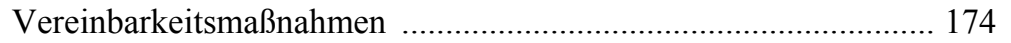

6.4.3 Karriereförderung über Weiterbildung und Prozessregelungen bei

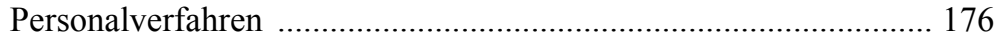

6.4.4 Frauenquoten als Schlüsselinstrument der Gleichstellung .............. 178

6.5 Fazit: Vorreiterrolle mit ungenutzten Potenzialen ......................... 179

$7 \quad$ Vorstellungen zu Gleichstellung und Geschlecht bei der Genese und Implementation betrieblicher

Gleichstellungspolitik am Beispiel der Schweiz ........................ 185

Gesine Fuchs, unter Mitarbeit von Yvonne Debrunner

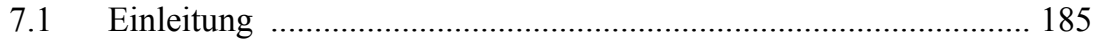

7.2 Geschlecht und Gleichstellung bei der Genese betrieblicher Gleichstellungspolitik ................................................................... 186

7.2.1 Betriebliche Gleichstellung und neues Bundespersonalrecht ......... 186

7.2.2 Konkretisierung der betrieblichen Gleichstellungspolitik: Parlamentarische Anträge 1996-2011 ......................................... 188

7.2.3 Frames für und gegen Gleichstellung in den Debatten zu betrieblichen Gleichstellungsmaßnahmen .................................... 192

7.2.4 Zusammenfassung: Eingespielte Debatten ohne offene normative Konflikte

7.3 Ziele der Gleichstellung in Programmen und Vorstellungen der Beschäftigten ............................................................................. 198

7.4 Gleichstellung durch Angleichung? ............................................. 200 
7.5 Direktion für Entwicklung und Zusammenarbeit (DEZA) als

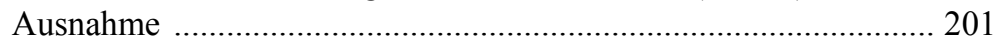

7.5.1 Lange Geschichte des Gender Mainstreaming .............................. 202

7.5.2 Gleichstellung durch Veränderungen der Institution ..................... 203

7.6 Fazit: Gleichstellungspolitischer Individualismus überwiegt ......... 206

8 Betriebliche Gleichstellungspolitik der Bundesverwaltungen im Ländervergleich .................................................................. 209

Silke Bothfeld, Gesine Fuchs, Andrea Leitner, Sophie Rouault

8.1 Bundesdienst - Enklave oder Vorreiter für die Beschäftigung hoch qualifizierter Frauen?

8.2 Substanzielle Maßnahmen der Gleichstellungsförderung:

Vereinbarkeit und Karriereförderung

8.2.1 Modernisierte Personalpolitik: Vereinbarkeit von Beruf und Familie im Bundesdienst ............................................................ 214

8.2.2 Förderung von Frauen in Führungspositionen: Wie sind direkte und indirekte Diskriminierung zu vermindern?

8.3 Institutionelle Maßnahmen für mehr Geschlechtergerechtigkeit in der Personalpolitik der Bundesverwaltung

8.3.1 Gleichstellungsbeauftragte: $\mathrm{Zu}$ schwach institutionalisiert für ihre Umsetzungsaufgaben

8.3.2 Gleichstellungspläne der Bundesministerien und Ämter: Strategisches Instrument für geschlechtergerechte Personalplanung

8.3.3 Berichterstattung zur Erhöhung der Verbindlichkeit von autonomem, aber zielgebundenen Handeln in der Bundesverwaltung

8.4 Fazit: Licht und Schatten in der Gleichstellungspolitik der drei Bundesverwaltungen

Literatur 237

Abkürzungen 


\section{Verzeichnis der Abbildungen}

Abb. 2.1: Verhältnis von (gleichstellungspolitischen)

Steuerungsregimen zu Policy-Regime und

Geschlechterregime

Abb. 3.1: Frauenanteile im Bundesdienst nach Aufgabenbereichen $(2011 / 2012$, in \%)

Abb. 3.2: Teilzeitarbeit im Bundesdienst und in der Gesamtwirtschaft nach Geschlecht (2011, in \%)

Abb. 3.3: Lohnunterschiede zwischen den Geschlechtern beim Bund und in der Gesamtwirtschaft (2010, in \%)

Abb. 3.4: Frauenanteil in verschiedenen Beschäftigungsgruppen im Bundesdienst (2010)

Abb. 4.1: Beschäftigung nach Lohnklassen, gesamte Bundesverwaltung (2002 und 2011)

Abb. 4.2: Frauenanteil in Prozent nach Lohnklassen, gesamte Bundesverwaltung (2002-2011)

Abb. 4.3: Frauenanteil in Prozent im mittleren Management (Lohnklassen 24-29) nach Departementen (2002 und 2011) ...

Abb. 4.4: Frauenanteil in Prozent im oberen Management (Lohnklassen 30-38) nach Departementen (2002 und 2011)

Abb. 5.1: Frauenanteil in den obersten Bundesbehörden (in \%)

Abb. 5.2: Teilzeitquote in den obersten Bundesbehörden (1992-2012, in \%)

Abb. 5.3: Frauenanteil nach ausgewählten Führungsfunktionen in obersten Bundesbehörden (1996-2002, in \%)

Abb. 6.1: Erwerbsquote und Teilzeitquote (1995-2013, in \%)

Abb. 6.2: Entwicklung des Frauenanteils und der Teilzeitquote im Bundesdienst (1995 bis 2013)

Abb. 6.3: Bundesbedienstete und Frauenanteil nach Berufsgruppen (2012)

Abb. 6.4: Frauenanteil nach Beschäftigungsverhältnissen und Berufsgruppen im Bundesdienst (1995 und 2013)

Abb. 6.5: Bundesbedienstete und Frauenanteil nach Qualifikationen (2013)

Abb. 6.6: Frauenanteil in den höchsten besoldungsrechtlichen

Einstufungen (2006-2013, in \%)

Abb. 6.7: Gender Pay Gap im Bundesdienst nach Berufsgruppen (2013)

Abb. 6.8: Veränderung des Frauenanteils in den höchsten

Besoldungsgruppen im Bundesdienst gesamt, BMASK und BMF (2007-2013) 


\section{Verzeichnis der Tabellen}

Tab. 3.1: Anteil der Beschäftigung in der öffentlichen Verwaltung an der Gesamtbeschäftigung $(1995,2001$ und 2011) .................... 45

Tab. 4.1: Beschäftigungsmuster in Prozent bei Familien mit Kindern unter 14 Jahren (2011)

Tab. 4.2: Frauenanteil in der Bundesverwaltung nach Departementen und in den untersuchten Ämtern (2002-2011)

Tab. 6.1: Bedienstete und Frauenanteil nach Ressorts (2013)

Tab. 6.2: Frauenbeschäftigung nach Beschäftigungsgruppen und Teilzeitbeschäftigung nach Ressorts (2013, in \%)

Tab. 7.1: Übersicht über gleichstellungsrelevante parlamentarische Geschäfte zur Gleichstellungspolitik in der Bundesverwaltung (1996-2011)

Tab. 7.2: Frames: Anzahl Verwendungen und Erfolgsrate in debattierten Vorstößen zur Bundesverwaltung

Tab. 8.1: Frauenbeschäftigung der Bundesdienste und in der Gesamtwirtschaft im Ländervergleich - zentrale Indikatoren (in \%) ...

\section{Verzeichnis der Tafeln}

Tafel 2.1: Untersuchte Verwaltungseinheiten und Analyseschritte

Tafel 3.1: Rechtlicher Rahmen für die Gleichstellung im Bundesdienst

Tafel 3.2: Unterschiedliche Statusprinzipien

Tafel 4.1: Rechtsgrundlagen betrieblicher Gleichstellungspolitik in der schweizerischen Bundesverwaltung

Tafel 4.2: Organisation der Gleichstellungsarbeit in der schweizerischen Bundesverwaltung

Tafel 4.3: Die vier untersuchten Ämter und ihre betriebliche Gleichstellungspolitik im Vergleich

Tafel 5.1: Gesetzliche Grundlagen der Gleichstellungspolitik im Bundesdienst

Tafel 5.2: Vergleichende Bewertung der Instrumente zur beruflichen Gleichstellung in der deutschen Bundesverwaltung

Tafel 7.1: Übersicht über Pro-Frames bei gleichstellungspolitischen Geschäften

Tafel 7.2: Übersicht über Contra-Frames bei gleichstellungspolitischen Geschäften

Tafel 8.1: Das Amt der Gleichstellungsbeauftragten im Drei-LänderVergleich 



\section{Betriebliche Gleichstellungspolitik des öffentlichen Dienstes als Forschungsfeld: Einleitung}

Silke Bothfeld, Gesine Fuchs, Andrea Leitner, Sophie Rouault

Erwerbsarbeit ist ein zentrales Feld für die Gleichstellung der Geschlechter, wird doch unter den gegenwärtigen politischen und ökonomischen Bedingungen zunehmend eine eigene Existenzsicherung aller erwerbsfähigen BürgerInnen unabhängig von Ehepartner, Vermögen oder Staat immer wichtiger. Trotz zahlreicher öffentlicher Bekenntnisse, vielfältiger politischer Maßnahmen und Rechtsgrundlagen zur Gleichstellung bestehen jedoch große Ungleichheiten zwischen den Erwerbs-, Einkommens- und Aufstiegschancen von Frauen und Männern fort, wie Studien zu Berufswahl, Lohnungleichheiten, sozialer Sicherung und Beschäftigungsstrukturen regelmäßig aufzeigen. Bei der Suche nach den Gründen für die relative Erfolglosigkeit von gleichstellungspolitischen Bemühungen fokussiert die Forschung vermehrt auf das Zusammenspiel einzelner Programme und Maßnahmen und die Dynamiken zwischen verschiedenen Politikbereichen: Welche Anreize gehen von einzelnen Maßnahmen aus, und sind diese untereinander konsistent? Legen sie ähnliche normative Leitvorstellungen zum Geschlechterverhältnis zugrunde oder widersprechen sich Norm, Logik und Anreiz?

Die Analyse beruflicher Gleichstellungspolitik in der Bundesverwaltung scheint aus mehreren Gründen relevant und lohnend. Als betriebliche Politik bietet sie ein überschaubares und abgrenzbares Feld und umfasst dabei Maßnahmen zur Beschäftigung (Zugang und Aufstiegsförderung), zur Sorgearbeit bzw. zur Vereinbarkeit von Familie und Beruf, aber auch prozedurale Instrumente, wie z.B. ein Monitoringsystem, sodass sich hier das Zusammenwirken verschiedener Maßnahmen gut analysieren lässt. Darüber hinaus ist der öffentliche Dienst ein breites, wichtiges und typisches Arbeitsfeld - rund $10 \%$ der Beschäftigten in den untersuchten Ländern sind hier in sehr unterschiedlichen Tätigkeiten tätig (vgl. Kapitel 3 in diesem Buch). Und schließlich hat der öffentliche Dienst Vorbildcharakter, sodass private Unternehmen von den hier gemachten Erfahrungen lernen können.

Gleichstellungspolitik in der öffentlichen Verwaltung hat zudem einen Doppelcharakter: Sie ist gleichzeitig eine genuin betriebliche Aktivität und als „normales“ Politikfeld Gegenstand politischer Interventionen, Debatten und Zielsetzungen, wie dies auch für andere Bereiche beruflicher Gleichstellungspolitik gilt. In der betrieblichen Gleichstellungspolitik legt der Gesetz-

1 Siehe dazu etwa als aktuelle Untersuchung Prognos (2014) für die Gesamtevaluation der ehe- und familienbezogenen Maßnahmen und Leistungen in Deutschland. 
geber die rechtlichen Rahmenbedingungen und Instrumente für das Handeln „seiner" Verwaltungen fest und nimmt damit eine Vorbildfunktion für andere Arbeitgeber und Unternehmen ein. Diesem Aspekt gilt unser besonderes Interesse: Wenn die Zentralverwaltung in einem Staat als Organisation Vorbildfunktion für andere Dienststellen und Betriebe hat und als öffentlicher Arbeitgeber der Umsetzung der Gleichstellungsgesetzgebung besonders verpflichtet ist, kann angenommen werden, dass gleichstellungspolitische Maßnahmen in der Bundesverwaltung sich leichter institutionalisieren lassen als in anderen Bereichen. Wir können davon ausgehen, dass sie hier besonders gut entwickelt und effektiv sind, sie also den Beschäftigten die Vereinbarkeit von Familie und Beruf ermöglichen, für den Zugang von weiblichen Beschäftigten in besonderem Maße offen sind und berufliche Karrieren von Frauen spezifisch fördern, um mittel- bis langfristig eine geschlechterparitätische Besetzung aller Leistungsstufen in der Organisation zu erreichen. Dementsprechend steht hier die Frage nach der (höchstmöglichen) Effektivität eines gleichstellungspolitischen Policy-Regimes im Fokus unserer Analysen.

Gleichstellungspolitik ist Querschnittspolitik. Sie weist rechtliche, ökonomische, soziale und kulturell-symbolische Aspekte auf, sodass Interaktionen zwischen den verschiedensten gesellschaftlichen Teilbereichen angenommen werden müssen. In der beruflichen Gleichstellungspolitik sind beispielsweise Politiken der sozialen Sicherung, der öffentlichen Finanzen, der Arbeitsmarkt-, Struktur- oder Bildungspolitik berührt. Im engeren Sinne werden mit Gleichstellungspolitik alle Entwürfe, Gesetze, Maßnahmen und Programme berücksichtigt, die auf eine gleichberechtigte Teilhabe von Frauen und Männern an bezahlter Arbeit zielen, besonders auf Zugang zu Erwerbsarbeit (Ausbildung, Arbeitszeit, Möglichkeiten der Kinderbetreuung, Freistellung) und auf Qualität der Beschäftigung (Aufgaben, Arbeitsbedingungen, Lohn, Weiterbildung, Aufstieg, Kündigung und Schutz vor sexueller Belästigung - vgl. von Wahl 2005: 68; Bothfeld et al. 2010: 37). Die ergänzende Betrachtung von allgemeinen Policies zur Erwerbs- und zur Betreuungsarbeit ist wesentlich, um die wechselseitigen Abhängigkeiten zwischen Produktion und Reproduktion, zwischen Erwerbs- und Sorgearbeit zu verstehen. Verflechtung und Interdependenzen der gesellschaftlichen Teilbereiche stellen damit hohe Anforderungen an staatliche Koordinierung und Steuerung, da diese unterschiedlichen Eigenlogiken folgen. Diese beinhalten immer auch normative Standpunkte, deren Identifikation und Analyse seit längerem Ziel feministischer Politikwissenschaft sind (Bothfeld/Kuhl 2008). Berufliche Gleichstellungspolitik berührt Grundsatzfragen zu Arbeitsteilung über Bewertung und über die Zuweisung der Geschlechter zu bezahlter und unbezahlter Arbeit. Berufliche Gleichstellungspolitik kann somit Sozialstaatsregime mitformen (vgl. Sainsbury 2013). Querschnittscharakter, Interdependenz sowie potenzielle Einflüsse auf wohlfahrtsstaatliche Arrangements machen für die berufliche Gleichstellungspolitik eine Untersuchung 
aus der Regimeperspektive sinnvoll und vielversprechend, da sich komplexe Prozesse und Wechselwirkungen zwischen gewandelten Geschlechterarrangements und staatlicher Politik analytisch und empirisch besser erfassen lassen als durch isolierte Maßnahmenbetrachtungen. Unsere Studie verstehen wir als einen Beitrag zur Feminist-Comparative-Policy-Literatur, die sich lange Zeit auf Agenda-Setting und Politikformulierung konzentriert hat und allmählich beginnt zu analysieren, was nach der Verabschiedung von Gesetzen und Programmen passiert (vgl. Mazur 2009; für die EU-Forschung vgl. Fuchs/Hinterhuber 2015). Die Studie kann auch anregen, für das Untersuchungsfeld vermehrt Implementationsanalysen durchzuführen. So haben viele Arbeiten Studien gezeigt, dass Regierungen die Umsetzung solch kontroverser transformativer Politiken häufig nicht aktiv vorantreiben und eher symbolische Maßnahmen verfolgen (vgl. Mazur o. J.: 2f.).

Wegen des Doppelcharakters der Gleichstellungspolitik öffentlicher Verwaltungen als betriebliche und als politisch-programmatische Aktivität begeben sich die AkteurInnen mit der Implementation der Gleichstellungspolitik in ein Spannungsverhältnis zu bestehenden Regeln und Strukturen - und vielleicht sogar ihren individuellen persönlichen Ansichten. Wir können daher die Probleme des öffentlichen Gestaltungsauftrags nicht verstehen, wenn wir nicht die betriebliche Praxis, hier: die Alltagspraxis in den Dienststellen der Bundesverwaltungen, als zentrale Implementationsbedingung berücksichtigen. Um die Gleichstellungspolitik in der Bundesverwaltung zu analysieren, verknüpfen wir darum organisationssoziologische und personalwirtschaftliche mit policyanalytischen Zugängen. Gerade diese Schnittmenge von betrieblicher Gleichstellung, staatlichem Handeln sowie Geschlecht und Organisation, die den Doppelcharakter der Gleichstellungspolitik der Bundesverwaltung ausmacht, ist bisher kaum erforscht. Betriebliche Gleichstellungspolitik in öffentlichen Verwaltungen ist bisher nur selten empirisch untersucht worden (so auch Strachan 2011: 347; siehe aber Engeli 2011). Studien sind eher anwendungsorientiert (z.B. OECD 2010), beziehen sich auf die USA oder Großbritannien (Naff/Kellough 2003; Conley/Page 2010; Wright/Conley 2011; Conley/Page 2014) oder betonen die organisationssoziologische, nicht die policyanalytischen Perspektiven (z.B. Andresen et al. 2003). Im Gegensatz dazu steht ein wachsender politikwissenschaftlicher Korpus über staatliche Gleichstellungsinstitutionen auch in vergleichender Perspektive. ${ }^{2}$ Gleichfalls dynamisch entwickelt sich wissenschaftliche und praxisorientierte Forschung zu betrieblicher Gleichstellung, wobei hier der

2 Für die Schweiz siehe Scheidegger (2008); Nentwich (2006); für Deutschland Lang (2007, 2009); Rudolph (2009); Rudolph/Schirmer (2004) und Wrangell (2012). Für Österreich siehe Appelt (2009). Die Ergebnisse aus dem Research Network on Gender and the State RNGS sowie aus dem QUING-Projekt sind komparativ (vgl. McBride/Mazur 2010; Haussman/Sauer 2007). 
analytische Fokus hier auf die Privatwirtschaft begrenzt bleibt. ${ }^{3}$ Vor dem Hintergrund des New Public Management gibt es vermehrt Untersuchungen zur Transformation der Arbeitsbedingungen in öffentlichen Verwaltungen, doch hier werden Geschlechteraspekte, die über quantitative Angaben hinausgehen, kaum einbezogen.

Die Studie ist daher grundsätzlich explorativ angelegt, um den oben konstatierten Doppelcharakter von betrieblicher Aktivität und „,normalem“ Politikfeld mit Vorbildcharakter aufzugreifen und ihre Wechselwirkungen zu problematisieren. Sie bietet zunächst eine Bestandsaufnahme der betrieblichen Gleichstellungspolitik in den Bundesverwaltungen Deutschlands, Österreichs und der Schweiz aus der Steuerungsperspektive. Bei der Auswahl unserer drei Länder folgen wir einem ,,most similar systems design “ (Lauth et al. 2009: 68-75), untersuchen also Fälle, die vielen Charakteristika gleich bzw. ähnlich sind, in wenigen Merkmalen sich aber unterscheiden, wobei diese Unterschiede für die unterschiedlichen Ergebnisse in der Steuerung der betrieblichen Gleichstellungspolitik verantwortlich sein können. Die Länder verfügen über ähnliche Kontextbedingungen (wie das föderale politische System oder Bürokratietraditionen), doch unterschiedliche Entwicklungspfade. Sie haben Geschlechterarrangements, die ähnliche Geschlechterkulturen (Werte, Normen, Leitbilder) und ähnliche Geschlechterordnungen (institutionelle Rahmenbedingungen, konservative bzw. liberal-konservative Wohlfahrtsregime) aufweisen. In den drei untersuchten Ländern hat sich zwar das männliche Ernährermodell modernisiert, nach dem der Ehemann weiterhin die Hauptlast der Erwerbsarbeit und die Ehefrau entsprechend die Hauptlast der Sorgearbeit trägt, doch kombinieren Frauen die Verantwortlichkeit mit jeweils unterschiedlichen Erwerbsmustern. Rechtspolitische Traditionen sind hingegen tendenziell unterschiedlich: Während in der Schweiz die Logik der individuellen Antidiskriminierung relativ stark verankert ist (etwa mit dem Gleichstellungsgesetz), stehen in Deutschland und Österreich die Erweiterung der Handlungsmöglichkeiten besonders für Frauen stärker im Vordergrund, etwa durch die Förderung längerer Auszeiten und des Wiedereinstiegs in den Beruf. Ein differenzierter Vergleich dieser unterschiedlichen Profile soll aufzeigen, welche spezifischen Handlungsmöglichkeiten für die Politik bestehen. Daher fragen wir:

3 Das Referenzwerk im deutschsprachigen Raum ist das von Gertraude Krell et al. herausgegebene „Chancengleichheit durch Personalpolitik“ (6. Auflage 2011). Dort werden öffentliche Verwaltungen vereinzelt als Fallbeispiele beschrieben. Liebigs Arbeiten zur Schweiz (2003, 2005, 2007, 2013) über betriebliche Geschlechterverhältnisse und Gleichstellungspolitiken behandeln die Privatwirtschaft. Ein laufendes Forschungsprojekt zur Schweiz, zu Deutschland und Österreich fragt, wie Unternehmen den wachsenden gesellschaftlichen Gleichstellungsdruck in ihrer Organisation bearbeiten (,Rekonfiguration von Geschlechterdifferenzierungen in Organisationen postmoderner Gesellschaften“; vgl. http://p3.snf.ch/ project-141954 - Zugriff: 30.04.2014 - sowie Funder 2014). 
1. Mit welchen Instrumenten und Maßnahmen wird betriebliche Gleichstellungspolitik innerhalb der öffentlichen Verwaltung und insbesondere in der Bundesverwaltung betrieben?

2. Wie greifen die Instrumente der Personalpolitik und der Gleichstellungspolitik des Bundesdienstes jeweils ineinander? Sind Spannungen zwischen der durch Tradition geprägten und durch die Gleichstellungsgebote herausgeforderten Strukturen gegeben? Wie wird ihnen jeweils begegnet?

3. Wie passen die tatsächlich vorfindlichen Maßnahmen im Steuerungsregime zueinander?

Das Ziel des Vergleichs ist die Analyse der Gemeinsamkeiten und Unterschiede der Regelungs- und Koordinierungslösungen in den drei Ländern. Welche Anregungen lassen sich daraus für eine wirkungsvolle Gleichstellungspolitik ableiten? Im zweiten Kapitel entwickeln wir unsere spezifische Analyseperspektive, in der wir zeigen, dass die „Unterfütterung“ der politikwissenschaftlichen Instrumentenperspektive mit Aspekten der feministischen Organisationsforschung und die Einbettung in die Regimeperspektive fruchtbar für die vergleichende Analyse von Steuerungsregimen ist. Wir gehen außerdem auf unseren methodischen Zugang zum Forschungsgegenstand ein. Im dritten Kapitel vergleichen wir die drei Bundesverwaltungen als Forschungsfeld anhand der zentralen Aspekte ihrer historischen Entwicklung, rechtlichen Verfasstheit, der Beschäftigungsbedingungen und anhand von Kernindikatoren zur Beschäftigung von Frauen und Männern im Bundesdienst. In den Kapiteln 4 bis 6 präsentieren wir die Fallstudien zu unseren drei Ländern Schweiz, Deutschland, Österreich, in denen wir jeweils auf den gesellschaftlichen Kontext, spezifische Regelungen sowie konkrete Instrumente eingehen, die nach institutionellen Regelungen, Vereinbarkeit von Familie und Beruf sowie Laufbahnförderung von Frauen geordnet sind. Hier sind auch Statistiken über das Personal verarbeitet, die sich nicht auf eine einheitliche vergleichbare Basis stellen ließen ${ }^{4}$ Das siebte Kapitel nutzt die breitere Datenerhebung in der Schweiz für eine exemplarische Analyse der Vorstellungen von Geschlecht und Gleichstellung, wie sie sich in Genese und Implementation der Politik in der Bundesverwaltung zeigen. Dabei geht es einerseits um Perspektiven und Ziele im parlamentarischen Politikformulierungsprozess sowie im Sinne der ,vergeschlechtlichten Organisation “ um Einstellungen der Beschäftigten, Gleichstellungsbeauftragten und Führungspersonen zur Gleichstellung der Geschlechter als Ziel der Organisation. Das achte Kapitel zieht den systematischen Vergleich zwischen unseren drei Länderstudien und zeigt die Unterschiede zwischen den drei Länderprofilen deutlich auf. Es bietet zusätzlich eine komprimierte Zusammenfassung der

4 Mehr vergleichbare Daten wären generell wünschenswert, weil sie ein zentrales Hindernis bei der vergleichenden Bürokratieforschung sind (vgl. Grunow 2009). 
vorangegangenen Kapitel und trägt Verbesserungspotenziale und weitergehende Forschungsfragen zusammen.

Die vorliegende vergleichende Studie ist aus dem schweizerischen Forschungsprojekt „Genese und Steuerung beruflicher Gleichstellungspolitik“ hervorgegangen, das im Rahmen des Nationalen Forschungsprogramms 60 „Gleichstellung der Geschlechter" durchgeführt wurde..$^{5}$ Das Projekt untersuchte Prozesse der politischen Agendagestaltung, der Politikformulierung und der Implementation entsprechender Policies seit Mitte der 1990er Jahre in der Schweiz. Es fokussierte vor allem auf drei Bereiche, nämlich die Gleichstellungsförderung bei öffentlichen Arbeitgebern, die Bereitstellung und Ausgestaltung von kommunalen Kinderbetreuungsangeboten und die Antidiskriminierungspolitik rund um das Gleichstellungsgesetz. Dabei stand die Generierung von Grundlagenwissen zu Herkunft, Dynamik und dem Zusammenspiel der zahlreichen Maßnahmen beruflicher Gleichstellungspolitik im Mittelpunkt. Dieses Zusammenspiel von Maßnahmen lässt sich durch betriebliche Gleichstellung öffentlicher Arbeitgeber und eine vergleichende Perspektive wie in diesem Buch besonders gut erforschen.

5 Genauer Projekttitel „Entstehung und Steuerung von schweizerischen Gleichstellungspolitiken zur Erwerbsarbeit“, Projekt-Nr. 129317, Projektteam: Thomas Widmer, Silke Bothfeld, Gesine Fuchs, Andrea Leitner, Sophie Rouault, Christine Zollinger (vgl. Widmer et al. 2014a sowie http://p3.snf.ch/project-129317, Zugriff: 12.01.2015). 


\section{Konzeptionelle Grundlagen und Untersuchungsdesign}

Silke Bothfeld, Gesine Fuchs, Andrea Leitner, Sophie Rouault

\subsection{Betriebliche Gleichstellungspolitik in der Bundesverwaltung als Teil der nationalen Geschlechterregimes}

Aus der Vogelperspektive ist die betriebliche Gleichstellungspolitik für den Bundesdienst ein relativ überschaubarer Teil eines Geschlechterregimes. Doch als politische Institution mit Vorbildfunktion trägt die Bundesverwaltung erheblich dazu bei, das gesellschaftliche Geschlechterverhältnis zu prägen, zu reproduzieren oder auch zu verändern. Dabei erlaubt der Regimebegriff in besonderem Maße, komplexe Prozesse und Wechselwirkungen zwischen den Geschlechterarrangements aller gesellschaftlicher Teilbereiche sowie die Einwirkungsmöglichkeiten öffentlicher Politik bzw. die Möglichkeiten und Grenzen öffentlicher Steuerung sichtbar zu machen (Betzelt 2007: 5f.). Ein Geschlechterregime ist sehr viel umfassender als unser Untersuchungsgegenstand, die Bundesverwaltung als Spezialgebiet der öffentlichen Verwaltung. So definieren Rosenberger und Sauer auf der Grundlage eines politikwissenschaftlichen Regimebegriffs als Geschlechterregime

„die informelle und formelle Herrschaftsorganisation politischer Macht entlang der Geschlechterlinie. Ein Geschlechterregime umfasst sowohl Institutionen, Organisationen wie auch Normen und Diskurse." (Rosenberger/Sauer 2004: 259)

Danach ist mit eigenständigen sozialen Dynamiken zu rechnen, die sich weitgehend staatlichen Steuerungsversuchen entziehen. Doch bezieht dieser Begriff eines Geschlechterregimes stärker politische Prozesse mit ein als es andere, soziologische Konzeptualisierungen von Geschlechterverhältnissen es leisten können. ${ }^{1}$ Vielmehr eröffnet sogar

„,[e]rst die Regimeperspektive (...) den Blick auf die Wirkung institutioneller Reformen auf die informelle Dimension (Praktiken und Identitäten) von Politik und (...) damit den Blick auf die Reichweite und Tiefe (geschlechter-)politischen Wandels.“ (Bothfeld 2008: 14)

Übertragen auf unseren Gegenstand bedeutet dies, dass sich die in der Bundesverwaltung handelnden Akteurinnen und Akteure in ihrer Alltagspraxis in einem Spannungsfeld bewegen, das sowohl durch die informellen - und

1 Diese fokussieren etwa unterschiedliche Arrangements von Erwerbsarbeit und Erwerbsbeteiligung, Care, Einkommensstrukturen, Zeitverwendung, ,voice“ und Beziehungsgestaltung mit ein (vgl. Betzelt 2007: 7). 
Abb. 2.1: Verhältnis von (gleichstellungspolitischen) Steuerungsregimen zu Policy-Regime und Geschlechterregime

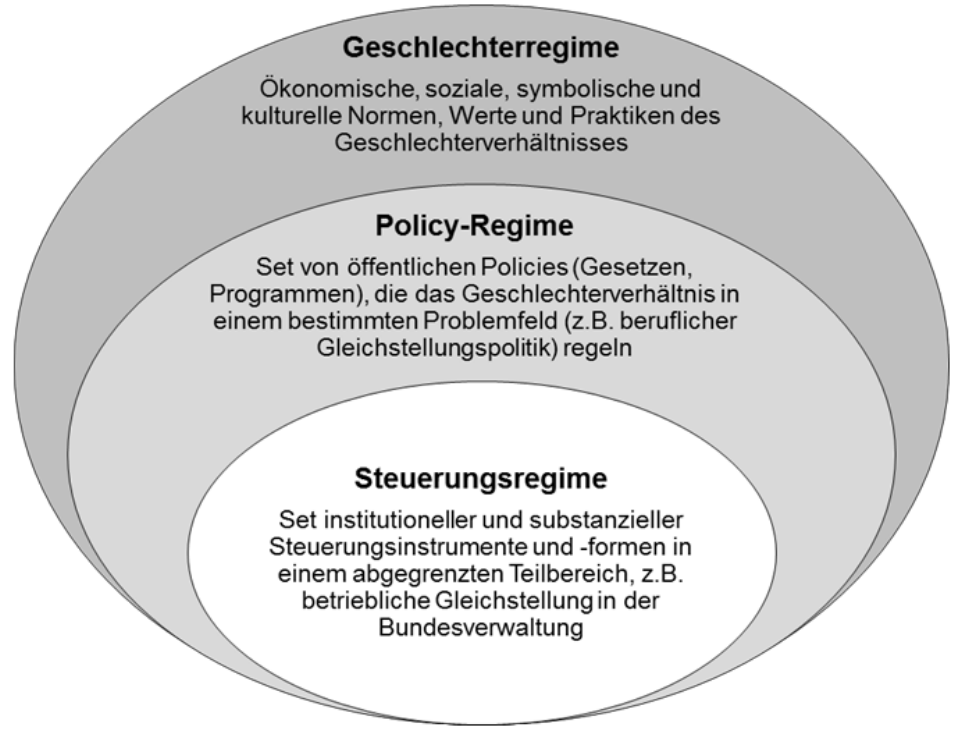

Quelle: eigene Darstellung

durch die Organisationsmitglieder reproduzierten - Geschlechterstrukturen geprägt ist, als auch durch die formalen gesetzlichen Regeln, die sich auf das Verwaltungshandeln beziehen.

- Als Geschlechterregime bezeichnen wir die ökonomischen, sozialen, symbolischen und kulturellen Normen, Werte und Praktiken des Geschlechterverhältnisses, die in der Gesamtgesellschaft präsent sind. Damit ist das Geschlechterregime gleichsam die ,äußere Hülle“ für das PolicyRegime der beruflichen Gleichstellungspolitik. Sie ist durch die Einheitlichkeit (bzw. Uneinheitlichkeit) der normativen Bezüge definiert und Quelle für normative Kohärenz in einer Gesellschaft.

- Als Policy-Regime bezeichnen wir das Ensemble von Vorschriften, Programmen oder Informationsmaßnahmen in einem bestimmten Politikfeld. Das Policy-Regime der beruflichen Gleichstellung umfasst drei zentrale Aspekte, nämlich die Gestaltung des Zugangs zur Erwerbsarbeit, die Qualität der Beschäftigung und die Durchmischung der Beschäftigung, wie sie etwa in Gleichstellungsgesetzen, dem Arbeitsrecht oder der Gestaltung der Kinderbetreuung zum Ausdruck kommen (Bothfeld et al. 2010). Policy-Regime beziehen sich somit immer auf ein bestimmtes Problemfeld (Erwerbstätigkeit, Schutz vor Gewalt, Pflegearbeit etc.), das durchaus mit 
anderen Feldern interagieren kann, und zeigen, wie gut die Maßnahmen zwischen den Teilbereichen (z.B. Aufstiegsförderung, Vereinbarkeit) miteinander abgestimmt wird. Im günstigen Fall sind die Teilbereiche gut koordiniert und das Policy-Regime somit konsistent.

- Ein Steuerungsregime ist ein Teilbereich eines Policy-Regimes. Es besteht aus einem Ensemble konkreter Steuerungsinstrumente und -modi, die in einem Teilbereich zusammenwirken, wie etwa bei der betrieblichen Gleichstellung in der Bundesverwaltung. An ihm können Umsetzung und mögliche Wirkungen von Gleichstellungspolitik unmittelbar analysiert werden. Hier kommt es auf die konkrete Ausgestaltung der Instrumente an, ob diese hinsichtlich des Steuerungsmodus, aber auch der Teilregelungen auf den Gegenstand der Steuerung so abgestimmt sind, dass sie ihre Wirkung entfalten können. ${ }^{2}$

Das Steuerungsregime der Gleichstellungspolitik in der Bundesverwaltung ist unser eigentlicher Untersuchungsgegenstand. Wir betrachten es aus der Regimeperspektive vor dem Hintergrund der anderen beiden größeren Dimensionen. Alle drei Ebenen interagieren miteinander nicht nur bei der Ausgestaltung der jeweiligen Policies, sondern auch im Hinblick auf die soziale Praxis: Erst in der Praxis wird deutlich, wie die Grundausrichtung eines Geschlechterregimes, die konkreten Anreize der einzelnen Maßnahmen eines Policy-Regimes und schließlich die Wirkungskraft der Instrumente zusammenspielen. Die Ebene der sozialen Praxis ist somit der „Prüfstein“ für die Akzeptanz und damit Effektivität der Policies.

Für die vergleichende Analyse der Ausgestaltung und Effektivität von gleichstellungspolitischen Steuerungsregimen sind aus dieser Regimeperspektive zwei Aspekte relevant: die Wechselwirkung verschiedener Policies innerhalb eines Policy-Regimes und die Passförmigkeit bzw. Angemessenheit der Instrumente. Schon allein dadurch, dass Steuerungsregime in jeweils unterschiedliche Kontexte eingebettet sind, kann ihre Effektivität, trotz ansonsten ähnlichen Instrumentenprofils, sehr unterschiedlich ausfallen. Damit verknüpft müssen beim Design der Steuerungsregime die aus den anderen beiden Dimensionen, dem Geschlechterregime und dem Policy-Regime, resultierenden Geschlechterverhältnisse berücksichtigt werden, also „Geschlechterwissen“ in Form von Forschungsergebnissen beim Design der Gleichstellungspolitik Anwendung finden und politische Instrumente entsprechend ausgestaltet sein.

2 Siehe hierzu die international vergleichende Analyse von Quotenregelungen für weibliche Aufsichtsratsmitglieder (Bothfeld/Rouault 2015). 


\subsection{Die Bundesverwaltung als vergeschlechtlichte Organisation und Teil eines Geschlechterregimes}

Wie die anderen Teilbereiche der schweizerischen, österreichischen oder deutschen Gesellschaften sind auch die Bundesverwaltungen von sozialen Strukturverhältnissen und damit auch vom Geschlechterverhältnis durchzogen: sie sind zunächst als eine grundsätzlich vergeschlechtlichte Organisation (,gendered organization“) zu verstehen. Hier wird das Argument von Sauer und Rosenberger, dass in der sozialen Praxis (hier: im Organisationskontext) formale und informelle Regeln der Herrschaftsorganisation das Geschlechterverhältnis bestimmen, besonders deutlich. Die britische Geschlechter- und Organisationssoziologin Joan Acker (1990) hat betont, dass die Handlungen und Wahrnehmungen der Organisationsmitglieder Auswirkungen auf die Reproduktion der Ungleichheit zwischen den Geschlechtern haben. Grundlegend gilt auch hier der - auch für andere wegweisende - Geschlechterbegriff der Historikerin Joan Scott, die das Merkmal Geschlecht nicht nur als Differenz, sondern auch als Machthierarchie begründend deutet:

„Gender is a constitutive element of social relationships based on perceived differences between the sexes, and gender is a primary way of signifying relationships of power." (Scott 1986: 1067)

Joan Acker, die sich auf die Analyse von Organisationen als einem der zentralen gesellschaftlichen Teilbereiche konzentriert hat, hat fünf analytische Dimensionen herausgearbeitet, wo Organisationshandeln geschlechtertypisiert ist (vgl. Acker 1990: 147):

- Die Konstruktion von Trennlinien entlang von Geschlecht - bei Arbeit, erlaubtem Verhalten, von physischen Orten sowie von Macht. In Letzteres schließt Acker die Institutionen ein, die Spaltungen in den Strukturen des Arbeitsmarktes, der Familie und des Staates aufrechterhalten;

- Konstruktionen von Symbolen und Bildern, die diese Aufspaltungen erklären, ausdrücken oder verfestigen. Diese Konstruktionen beruhen auf vielfältigen Quellen in der Sprache, Ideologie, Populär- und Hochkultur, in der Kleidung oder den Medien;

- Interaktionsprozesse zwischen Frauen und Männern, Frauen und Frauen sowie Männern und Männern, die vergeschlechtlichte soziale Strukturen (und Organisationen) hervorbringen, einschließlich aller Muster, die Dominanz und Unterwerfung ausüben (nach Acker 1990: 146f.);

- die Produktion vergeschlechtlichter Komponenten individueller Identität (die z.B. für Berufswahl und Karriereorientierung relevant ist);

- die Einbettung von Gender in die größeren Konstitutionsprozesse sozialer Strukturen, von Familie bis komplexer Organisationen - was auf das umfassende Konzept des Geschlechterregimes verweist, welches wir hier nicht behandeln. 
Diese Vergeschlechtlichungsprozesse in Organisationen können offen oder verdeckt sein; Acker hat dafür den Begriff der „gendered substructure“ benutzt:

„,Gendered Substructure " points to often-invisible processes in the ordinary lives of organizations in which gendered assumptions about women and men, femininity and masculinity, are embedded and reproduced, and gender inequalities perpetuated. The most common inequalities are the wage gap between women and men and the sex segregation of jobs, occupations, and hierarchical positions.“ (Acker 2012: 215)

Die „vergeschlechtlichte Substruktur“ ist eng auf die geschlechtsspezifische Arbeitsteilung, etwa die selbstverständliche Zuschreibung von Reproduktions- und Care-Arbeiten an Frauen bezogen. Somit stehen formelle und informelle Arrangements zur Vereinbarkeit von Familie und Beruf, zu Telearbeit oder Urlaubsregelungen im Zentrum dieser Substruktur. Die Ausgestaltung solcher Arrangements in einer Organisation, etwa einer Einheit der Bundesverwaltung, kann zeigen, wie das Verhältnis von Erwerbs- und Reproduktionsarbeit gedacht wird: Sind Beschäftigte autonome Individuen ohne Sorgeverpflichtungen oder ist Sorgearbeit integraler Teil ihres Daseins? Wem werden solche Pflichten implizit oder explizit zugeschrieben? Geschlechterstereotype Substrukturen können auch bei Fragen der Karriereentwicklung, bei Mitarbeitendengesprächen, Sanktionierung geschlechtsuntypischen Verhaltens, bei internen Netzwerken oder bei Politiken zur Weiterbildung wirksam werden und betreffen damit alle Beschäftigte unabhängig von ihrer konkreten Situation.

Der Charakter der vergeschlechtlichten Substrukturen der Bundesverwaltung wird Einfluss auf die Implementationschancen der Gleichstellungspolitik haben. Für eine Analyse der Steuerung gleichstellungspolitischer Maßnahmen sind darum die in programmatischen Dokumenten enthaltenen (impliziten) Leitbilder wichtig, ebenso die tatsächlichen Praktiken: So könnte Teilzeitarbeit eine Abwertung erfahren, wenn etwa weiter althergebrachte Leistungs- und Arbeitsethiken der ständigen Verfügbarkeit eines männlichen Vollzeitarbeitnehmers ohne Sorgeverpflichtungen bei Vorgesetzten und KollegInnen vorherrschen (Koch 2007).

Eine Studie zu Verwaltungsmodernisierung und Gender Mainstreaming unterstreicht die Bedeutung eines bestimmten „Geschlechterwissens“ für das Handeln in Organisationen. Sünne Andresen und Irene Dölling (2005) haben gezeigt, dass die eigentliche Implementation gleichstellungspolitischer Maßnahmen von Machtverhältnissen und Interessendivergenzen abhängt und davon, welche Deutungsmuster sich in damit verbundenen Aushandlungsprozessen durchsetzen können. Sie haben zudem herausgearbeitet, dass das sog. Geschlechterwissen der Beteiligten als eine Form des Alltagswissens handlungsleitend ist. Andresen und Dölling definieren Geschlechterwissen als Begriff 
„für die verschiedenen Arten kollektiven Wissens, die in einer Gesellschaft jeweils über den Geschlechterunterschied, die Begründungen seiner ,Selbstverständlichkeit', und Evidenz, die (vor-)herrschenden normativen Vorstellungen und über die ,richtigen' Beziehungen und Arbeitsteilungen zwischen Frauen und Männern kursieren." (Andresen/Dölling 2005: 175)

Als „habitualisiertes Klassifizierungsmuster“ ist diese spezifische Art von Wissen nicht einfach durch Aufklärung oder Gendertrainings veränderbar. Andere Formen des Geschlechterwissens, etwa institutionell hervorgebrachtes Wissen (z.B. aus Religion oder Recht), wissenschaftliches Wissen oder ExpertInnenwissen zur Gleichstellungsarbeit (vgl. Ostendorf 2011: 8) stehen in einem komplexen Verhältnis zueinander (vgl. Wetterer 2002, 2011). Es ist also damit zu rechnen, dass die für Organisationen typischen Phänomene der geschlechtlichen Strukturierung auch bei der Bundesverwaltung zum Tragen kommen. Anders als in privaten Organisationen und Unternehmen ist die Organisation der Arbeitsprozesse hier relativ zuverlässig und engmaschig gesetzlich und/oder tariflich geregelt, sowohl durch die verschiedenen allgemeinen personalrechtlichen Gesetze für den öffentlichen Dienst als auch teilweise durch spezielle Gleichstellungsgesetzgebung im öffentlichen Bereich.

Aus diesen Überlegungen folgt, dass es zwei Untersuchungsperspektiven braucht, die miteinander verbunden werden sollten: Ein Blick auf konkrete Steuerungsregime und ihre ,technische“ Ausgestaltung muss verknüpft werden mit einem Blick auf konkrete Bedeutungsgehalte von Gleichstellung und Geschlecht, der versucht, Leitbilder und Konstruktionen nachzuvollziehen. Dieser Anforderung können wir hier nur eingeschränkt entsprechen: Das siebte Kapitel analysiert Auffassungen und Bezugsrahmen zu Geschlecht und Gleichstellung in der schweizerischen Bundesverwaltung.

Aufgrund der besonderen Rolle der Bundesverwaltung - sie gehört zur Exekutive, und ist somit als Teil der Bundesregierung eine politische Institution - sind diese Organisation und auch ihre innere Struktur gut von außen nachvollziehbar. Als politische Institution hat sie eine Vorbildfunktion, da sie Leitideen für die Organisation von Arbeitsprozessen nach außen hin sichtbar macht. Die politikwissenschaftliche Institutionentheorie geht davon aus, dass politische Institutionen - im Sinne von Organisationen - verbindliche, auf gesamtgesellschaftliche Relevanz zielende Entscheidungen herstellen, sie ordnen und durchführen. Es geht also einerseits um einen Willensbildungsprozess, andererseits um die Ausführung der Entscheidungen durch die Verwaltung (vgl. Göhler 1997: 17f.). Verbindlichkeit und gesamtgesellschaftliche Relevanz grenzen politische Institutionen von anderen sozialen Institutionen ab. Was ,gesamtgesellschaftlich relevant" werden soll und wird, ist Gegenstand von politischen Konflikten und Agenda-Setting-Prozessen. Zudem steuern politische Institutionen nicht nur, sie dienen auch der gesellschaftlichen Integration, also der Orientierung von BürgerInnen oder Adres- 
satInnen an bestimmten Werten und Ordnungsprinzipien - beispielsweise der Orientierung an internationalen Menschenrechtsstandards. Orientierungsleistungen wirken erst dann, wenn sie auf Dauer gestellt sind oder wenn sich Handlungsmuster in der Politik durchsetzen, die allmählich zu ,geronnenen Strukturen mit präskriptiver Wirkung“ werden (Göhler 1997: 25). In Institutionen schlagen sich also die Orientierungsleistungen nieder und sie bringen Sinn- und Wertorientierungen in die Politik ein:

„Politische Institutionen sind Regelsysteme der Herstellung und Durchführung verbindlicher, gesamtgesellschaftlich relevanter Entscheidungen und Instanzen der symbolischen Darstellung von Orientierungsleistungen einer Gesellschaft. Als solche sind sie sowohl festgelegter Rahmen als auch geronnene Muster des Handlungsraums Politik." (Göhler 1997: 26)

Staatliche Organisationen, die auf die Produktion verbindlicher, gesellschaftlich relevanter Entscheidungen ausgelegt sind, determinieren daher das Handeln ihrer Mitglieder nicht nur über ihren Organisationszweck mit ihren internen Regeln, sondern auch anhand der Leitideen, die ihr eigenes Handeln anleiten. Nicht nur mit den politischen Inhalten (z.B. einem Gesetzesprojekt zur Frauenquote), sondern auch mit ihrer internen Organisation, die auch ihre Personalpolitik umfasst, transportiert eine Organisation auch geschlechterpolitische Leitbilder nach außen (Henninger/Ostendorf 2005: 11-17). Diese indirekte Verhaltenssteuerung, die sich aus der Orientierungsfunktion einer Institution ergibt, kann zur normativen Integration eingesetzt werden, d.h. zur Herstellung einer Übereinstimmung zwischen politischen Zielen und normativen Orientierungen der Bevölkerung (Bothfeld 2008: 12f.). Mit anderen Worten hat die Gleichstellungspolitik bzw. Gleichstellungserfolge der Bundesverwaltung eine Signalfunktion für andere Dienststellen und Betriebe. Der Doppelcharakter der Bundesverwaltung als Organisation und Teil eines Geschlechterregimes ist daher erforderlich für das Verständnis von Gleichstellungspolitik, da einerseits die Dienststellen der Bundesverwaltung als ,gendered organizations" verstanden werden können, andererseits die Bemühungen, hier das staatliche Ziel der Gleichstellung gut umzusetzen, aufgrund der Vorbildfunktion besonders weitgehend sein dürften.

\subsection{Das Policy-Regime der beruflichen Gleichstellung}

Betriebliche Gleichstellungspolitik ist innerhalb der gesamten beruflichen Gleichstellungspolitik nur ein Teilbereich, und dieser kann als Policy-Regime untersucht werden. Ein (gleichstellungspolitisches) Policy-Regime ist ein Konzept der institutionalistischen Analyseperspektive, das ein Set von öffentlichen Policies (Gesetzen, Programmen) bezeichnet, die zum Ziel haben, in einem bestimmten Problemfeld (z.B. beruflicher Gleichstellung) Verände- 
rungen herbeizuführen. Der Verweis auf das Geschlechterregime unterstreicht, dass auch die Maßnahmen zur beruflichen Gleichstellung immer im Kontext anderer Strukturen betrachtet werden müssen, da viele Policies zusammenwirken und das Geschlechterverhältnis im Arbeitsmarkt gemeinsam bestimmen. Das Akteursverhalten wird jedoch nicht ausschließlich im Betrieb beeinflusst: In der beruflichen Gleichstellungspolitik sind vor allem sozialpolitische Regelungen wichtig, die ähnliche oder unterschiedliche Anreize zur Erwerbstätigkeit von Frauen und Männer setzen können, und eben übergreifende normative Handlungsbedingungen des Geschlechterregimes, die die Erwerbstätigkeit und Karriereorientierung als Handlungsoption für Frauen vorsehen können oder nicht. Für die betriebliche Gleichstellungspolitik des öffentlichen Dienstes ist außerdem der Bereich der Personalpolitik wichtig, der die Beschäftigungsbedingungen und -verläufe im Grundsatz strukturiert und die Dienste sowie beruflichen Karrieren in diesem Bereich für Frauen und Männer unterschiedlich ausgestaltet.

Geschlechtergleichheit ist ein mehrdimensionales Konzept. Wir orientieren uns hier normativ an Nancy Frasers einflussreichen Text (2000) zur „Gleichheit der Geschlechter und das Wohlfahrtssystem“. Sie formuliert normative Anforderungen an eine umfassende Gleichstellungspolitik: Sie soll Armut bekämpfen, für eine Gleichverteilung von Einkommen, Freizeit und Achtung sorgen, die soziale wie politische Marginalisierung von Frauen beenden und schließlich den Androzentrismus bekämpfen, d.h. die Ausrichtung an heute hegemonialen männerzentrierten Auffassungen über Arbeit und Leben. Eine solche Politik muss Aspekte der sozialen Sicherung, der öffentlichen Finanzen, der Arbeitsmarkt-, Struktur- oder Bildungspolitik aufnehmen. Sie muss Grundsatzfragen zur Arbeitsteilung über die Bewertung und über die Zuweisung der Geschlechter zu bezahlter und unbezahlter Arbeit stellen. Fraser entwickelt ein anspruchsvolles Integrationsmodell, das darauf gerichtet ist, dass alle Erwachsenen Erwerbs- und Sorgearbeit verrichten und gut miteinander verbinden können. Damit sind die Anforderungen für eine umfassende und in sich widerspruchsfreie Gleichstellungspolitik sehr hoch. Ein Policy-Regime zur beruflichen Gleichstellung (das wiederum nur ein Teilbereich umfassender Gleichstellungspolitik ist) muss sich mit den genannten drei Aspekten des Zugangs, der Qualität der Beschäftigung und der Durchmischung der Geschlechter beschäftigen.

Die Vielzahl und Unterschiedlichkeit der AdressatInnen und deren Handlungslogiken in diesen drei Bereichen stellen besondere Anforderungen an das Policy-Regime, damit es konsistent und auch kohärent ist. Grundsätzlich ist nämlich die Auswahl und Durchsetzung von konkreten Maßnahmen und Instrumenten eine komplexe Aktivität, die hohe Anforderungen stellt:

„Instrument choices, to be efficient, must be closely and carefully related to policy goals, and (...) any new goals and tools must also be carefully integrated with existing policies if implementation is to succeed. New and old goals must be coherent in the sense of being 
logically related, while new and old instrument choices must be consistent, in the sense of not operating at cross-purposes." (Howlett et al. 2009: 172)

Doch damit nicht genug - nicht nur müssen bestehende und neue Politikziele logisch miteinander verknüpft sein, sondern die Instrumente müssen angemessen im Hinblick auf Wertvorstellungen und soziale Realitäten sein, damit eine Politik den angestrebten Verhaltenswandel auch erreichen kann. Kohärenz verstehen wir darüber hinaus in diesem Sinne:

„Kohärenz setzt Konsistenz voraus, verlangt aber zudem, dass der Bezug zur (mehrheitlichen) sozialen Praxis bzw. den dominierenden sozialen Identitätsstrukturen gegeben ist. Kohärenz meint also die normative und ,technische' Passförmigkeit der Politikinstrumente und des politischen Paradigmas mit den sozialen Gegebenheiten (der, Rechtswirklichkeit'). Kurz gesagt, Konsistenz bezieht sich auf die ,sozialtechnologische“ Umsetzung von PolicyIdeen in Instrumente und deren Effektivität, Kohärenz dagegen auf die Angemessenheit der Instrumente im Hinblick auf die soziale Realität sowie deren normative Akzeptanz." (Bothfeld 2008: 13)

Konsistente Maßnahmen widersprechen sich nicht, entwickeln also keine gegensätzlichen Anreizwirkungen, sondern streben zusammengenommen ein einheitliches Verhalten bei den Zielgruppen an, etwa die Steigerung der Erwerbstätigkeit von Frauen (ebd.). Nun kann etwa der im öffentlichen Dienst in Deutschland und Österreich bestehende Rechtsanspruch auf langjährige Freistellungen wegen Familienarbeit jedoch den Fördermaßnahmen für Frauen beim beruflichen Aufstieg entgegenwirken: Sie sind somit inkonsistent mit Maßnahmen der Karriereförderung, solange die Freistellungsphasen in der Praxis nicht gleichermaßen von Männern beansprucht wird.

Andererseits zählt bei der Kohärenz auch die Einheitlichkeit der normativen Ausrichtung eines Policy-Regimes. Denn nur, wenn ein normatives Ziel, wie etwa das Zweiernährermodell als Bezugspunkt für alle Maßnahmen gilt und zugleich auch die verschiedenen Bedürfnisse und Handlungslogiken der AdressatInnen (wie z.B. Einstellungen oder Verdienstunterschiede) berücksichtigt werden, kann sich die beabsichtigte Steuerungswirkung entfalten (Kohärenz).

Die Vorstellung eines konsistenten und kohärenten Policy-Regimes ist natürlich idealtypisch, in der Realität weisen Policy-Regime immer Brüche und Widersprüchlichkeiten auf und sind damit immer nur mehr oder weniger effektiv (siehe hierzu ausführlicher Bothfeld 2008). Fragmentierungen bergen das Problem, dass - möglicherweise nicht intendiert - für unterschiedliche soziale Gruppen (Frauen/Männer oder Gering-/Hochverdiener) unterschiedliche Anreizwirkungen erzielt werden und sich systematische Differenzen in den Verhaltensweisen herausbilden. Bei mangelnder Konsistenz können sich die Wirkrichtungen verschiedener Instrumente hingegen gegenseitig aufheben, sodass im Ergebnis Mitnahmeeffekte oder nicht intendierte Wirkungen entstehen können. 
Dies gilt im besonderen Maße für das Zusammenspiel mit den Regeln der Personalpolitik im öffentlichen Dienst. Hier wirken sich formale Regelungen sehr unterschiedlich auf Frauen und Männer aus. Sie fördern und institutionalisieren damit die Ungleichheit zwischen Frauen und Männern. Hierzu gehören vor allem die Maßnahmen zur Vereinbarkeit von Beruf und Familie, aber auch die Regeln zu Entlohnung und beruflichem Aufstieg.

\subsection{Das Steuerungsregime der Gleichstellung in der Bundesverwaltung}

Steuerung und Implementation entscheiden darüber, ob die Ziele einer Policy tatsächlich auch erreicht werden können. Das empirisch vorfindliche spezifische Ensemble aus einzelnen Maßnahmen, das auf die Gleichstellung in der Bundesverwaltung gerichtet ist, bezeichnen wir daher als Steuerungsregime. Das Steuerungsregime verweist auf die Frage, mit welchen Modi gesteuert wird und wie die Instrumente im Einzelnen ausgestaltet sind (siehe Bothfeld/ Rouault 2015). Dabei ist die Wahl der Instrumente und Steuerungsmodi bei der Politikformulierung nicht zufällig, sondern insgesamt ein anspruchsvoller und konfliktanfälliger Prozess, in dem Ideen, Interessen und institutionelle Traditionen eine große Rolle spielen (für einen Forschungsüberblick vgl. Peters 2002 und Howlett et al. 2009: 114-135). Konkrete Instrumente transportieren dabei immer auch ein bestimmtes Verständnis der Beziehung zwischen dem Staat und seinen BürgerInnen sowie von sozialer Kontrolle (Lascoumes/Le Galès 2007).

Die politikwissenschaftliche Steuerungstheorie unterscheidet hierzu verschiedene Modi, die auf die Grundformen Regulierung, Finanzierung und Überzeugung zurückgehen (siehe Lowi 1972; hierauf Bezug nehmend: Benz, $2008)^{3}$. Steuerung sowie Steuerungsmöglichkeiten haben sich in den letzten Jahrzehnten stark verändert, dennoch bleibt der Begriff der Steuerung unverzichtbar, wenn er als ,intentionale Machtausübung“ (Göhler 2010: 36) analysiert werden soll. ${ }^{4}$ Regulierung kann z.B. ein gesetzliches Diskriminierungsverbot sein, eine finanzielle Anreizsteuerung kann durch das Elterngeld versucht werden, und Information oder Überzeugung kann durch Broschüren, Webauftritte und Ähnliches umgesetzt werden. Der Staat kann zudem Rahmenbedingungen setzen, bei denen private Akteure miteinander kooperieren (z.B. das Zusammenspiel der Regulierung in der Arbeitszeitgestaltung zwi-

3 Verschiedene Klassifikationen finden sich bei Windhoff-Héritier (1987); Schubert (1991); Howlett et al. (2009: 117-135) und Hood/Margetts (2007).

4 Göhler verortet Steuerung zwischen den Polen harter (hierarchischer) und weicher (horizontaler) Steuerung und systematisiert weiche Steuerung anhand der Prinzipien Diskurs, Fragen und Argumente sowie Symbole. 
schen Gesetz, Tarifvertrag und Betrieb). Beim sog. reflexiven Recht werden weniger materielle und sanktionsfähige Vorschriften, sondern eher Verfahren für die Erreichung eines bestimmten Ziels festgelegt und überprüfbar gemacht. ${ }^{5}$ In der deutschen Gleichstellungspolitik ist das Prinzip des reflexiven Rechts anhand eines Entwurfs eines Gleichstellungsgesetzes für die Privatwirtschaft diskutiert worden, wonach Unternehmen dazu verpflichtet worden wären, einen selbst gewählten Gleichstellungsplan umzusetzen, und bei Nichterfüllung (unzureichende Zielsetzungen oder Umsetzungsaktivitäten) in einer zweiten Stufe vorgeschriebene Maßnahmen aus dem Gesetz zum Zuge gekommen wären (Pfarr 2001). Kooperationen, freiwillige Vereinbarungen oder Prozesse des „Politiklernens“ können in Bereichen wirksam werden, in denen die AdressatInnen sich der Reichweite etwaiger Sanktionen tendenziell entziehen können - oder es schlichtweg keine gesetzliche Regelung gibt, AkteurInnen jedoch ein Interesse an der Fortentwicklung einer Policy haben. ${ }^{6}$ Gerade für die Gleichstellungspolitik der öffentlicher Verwaltung und der Exekutive ist der Aspekt des politischen Lernens ein zentraler, wenngleich indirekter und inkrementeller Steuerungsmodus (für das Beispiel Gender Mainstreaming vgl. Bothfeld 2005). Die umfassende, direkte und unverzügliche Wirkung einer gesetzlichen Regelung können diese Modi jedoch kaum ersetzen. $^{7}$

Für die vergleichende Analyse der öffentlichen Verwaltung, die Teil der Exekutiven ist, bietet sich die heuristische Unterscheidung zwischen institutionellen und substanziellen Politiken an. ${ }^{8}$ Unter institutioneller Politik wird die Schaffung von institutionellen Regeln für das Handeln von AkteurInnen in einer Organisation verstanden, welche selbst aber keine konkreten Lösungen vorgeben. Diese sind in Organisationen immer auf die Beziehung ver-

5 So gelten etwa die deutsche Umsetzung des Equal-Pay-Prinzips für die Leiharbeit oder ganz allgemein die Tarifpolitik als arbeitsrechtliche Beispiele für eine reflexive Regulierung, weil die AdressatInnen zum Handeln aufgefordert werden, der Staat jedoch die Letztverantwortung für die Realisierung der gesetzlichen Ziele (gleiche Bezahlung für LeiharbeitnehmerInnen, ,gute“ Arbeitsbedingungen) innehat (vgl. Kremer/Bothfeld 2012). Zu reflexivem Recht vgl. Hagenah (1996); Raasch (1999).

6 Vgl. Bothfeld (2008) sowie als Überblick Maier/Nullmeier (2003).

7 So hat die ersatzweise „freiwilligen Vereinbarung“ zwischen der Bundesregierung und der Wirtschaft zur Förderung von Frauen in den jährlichen keine substanziellen Erfolge gezeitigt (Lepperhoff 2010). Dasselbe gilt für die Förderung von Frauen in Führungspositionen in der Privatwirtschaft (Bothfeld/Rouault 2015).

8 An anderer Stelle, bei der vergleichenden Analyse der Quotenregelung, haben wir die Steuerungsformen anhand der Anforderungen an die AdressatInnen unterschieden und dabei hierarchische, prozedurale und evaluative Maßnahmen identifiziert (Bothfeld/Rouault 2015). Der Fokus war hier, die Effektivität einzelner Quotenregelungen vergleichend zu bewerten. Die Unterscheidung zwischen institutionellen und substanziellen Policies ist für den Vergleich von ganzen Policy-Regimes praktischer, weil es zunächst um das Verständnis ihrer Funktionsweisen geht. 
schiedener Akteursgruppen untereinander bezogen. ${ }^{9}$ Substanzielle Politik bezeichnet staatliches Handeln in Form von Regulierung, Förderprogrammen oder Informationskampagnen, die konkrete inhaltliche Vorstellungen transportieren. Sie zielt auf die unmittelbare Lösung eines gesellschaftlichen Problems und auf die Veränderung des Verhaltens der AdressatInnen (Sager 2009: 361; Knoepfel 2000: 285-286). Auch wenn keine konkreten Ziele formuliert werden, ist institutionelle Politik nicht leichter durchzusetzen als substanzielle Maßnahmen, denn mit der Veränderung des organisatorischen Institutionengefüges verschiebt sich auch die Machtverteilung zwischen den AkteurInnen in organisationsinternen Handlungsprozessen.

Wir vermuten einen wesentlichen Einfluss von institutioneller auf den Umfang und die Umsetzung substanzieller Politik: Institutionelle Politik besorgt die Feinabstimmung der Regeln, nach denen die öffentlichen (und zum Teil privaten) AkteurInnen in die Umsetzung der gleichstellungspolitischen Ziele einbezogen werden und nach denen sie handeln sollen. Sie legen zudem Ressourcen und Befugnisse der Akteure fest. Im Kontext der Bundesverwaltung sind z.B. Gleichstellungbeauftragte und Gleichstellungspläne prominente Instrumente institutioneller Politik, welche der Kontrolle der Leitung in Personalfragen dienen und deren Handlungsoptionen damit kontrollieren und gegebenenfalls auch einschränken. Interventionen von Gleichstellungsbeauftragten werden daher zuweilen auch als Störfaktor in regelhaften bürokratischen Prozessen kritisiert, besonders dann, wenn sie die Prozesse als nicht regelkonform beanstanden oder ihre Informations- oder Mitbestimmungsrechte einfordern (siehe auch Geppert/Lewalter 2012). Arbeitszeitregelungen, Kinderbetreuung oder Coachingprogramme definieren wir hier hingegen als Instrumente substanzieller Politik, da diese die Arbeitsbedingungen der Beschäftigten direkt verändern und Frauen die Wahrnehmung ihrer beruflichen Chancen ermöglichen sollen. Substanzielle Maßnahmen kosten zumeist Geld, sind im Hinblick auf die Strukturen zwischen den Organisationsmitgliedern jedoch zunächst neutral. Bei der institutionellen Politik sind die AdressatInnen die Leitungsfunktionen in der Organisation, bei der substanziellen Politik sind es die Beschäftigten.

Die Integration neuer Aufgaben (wie Gleichstellung) kann daher im $\mathrm{Zu}$ sammenspiel mit den zuvor vorhandenen Aufgaben und institutionellen Policies (wie Personalpolitik) eine besondere Herausforderung darstellen. Am Beispiel der Länderfallstudien wird deutlich werden, welche Spannungen die Implementation gleichstellungspolitischer Maßnahmen mit den Regeln und Verfahren der Personalpolitik produzieren kann.

9 Genauer ist sie bezogen auf den Aufbau, die Stabilisierung oder Veränderung einer organisierten Akteursgemeinschaft, die in der Lage ist, konkrete Probleme zu lösen, sog. mikroinstitutionelle Politiken (vgl. Knoepfel 2000). Institutionelle Politiken auf der Ebene des Staatswesens hingegen wären z.B. die Organisation des Föderalismus, Wahlrecht oder direktdemokratische Verfahren. 
Schließlich postulieren wir drei „, Gütekriterien“, die gute Wirkungsvoraussetzungen für eine effektive institutionelle und substanzielle Politik darstellen: der Grad der Verbindlichkeit einer Maßnahme, ihre Reichweite und ihre Ausdifferenziertheit (vgl. Bothfeld et al. 2010; siehe auch Bothfeld/Rouault 2015).

Die Verbindlichkeit einer Maßnahme hängt davon ab, welche Folgen die Nichteinhaltung einer Vorschrift für die AdressatInnen haben kann: Je einschneidender die mögliche Sanktion, desto wahrscheinlicher ist ein Bemühen um Einhaltung der Vorschrift. Sanktionen können dabei materieller oder nicht-materieller Natur sein. So kann, wenn ein Arbeitgeber ein ihm in einem Einstellungsverfahren unterstelltes Diskriminierungsverhalten nicht widerlegen kann, unmittelbar gegen den Regelverstoß geklagt und der Arbeitgeber $\mathrm{zu}$ einer Entschädigung gezwungen werden. Voraussetzung ist dabei allerdings, dass die Regel klar und konkret definiert ist (Verbot der Diskriminierung), ihre Erfüllung überprüfbar und messbar ist und diese mit einem Klageund Kontrollrecht ausgestattet ist. Nicht-materielle Sanktionen sind nicht minder wirkungsmächtig, wenngleich die Regulierung dann als ,weich“ gilt (siehe auch Göhler 2010). Sie bestehen z.B. darin, dass die Nichtbefolgung einer anerkannten Norm öffentlich (gemacht) wird und die Sichtbarkeit des Regelverstoßes in einer für die Organisation wichtigen Öffentlichkeit oder ein Vergleich zwischen Peers (anderen Organisationen) für diese Organisation ,politische“ oder ökonomische Kosten verursacht. ${ }^{10}$ Die Verbindlichkeit hängt somit nicht allein vom Vorhandensein von Sanktionsmöglichkeiten ab, sondern von der Möglichkeit, den Regelverstoß als solchen öffentlich sichtbar zu machen und normabweichendes Verhalten zu problematisieren. Informell erfolgt dies über (teil-)öffentliche Kommunikationsprozesse, formal kann dies über die Verknüpfung einer Vorschrift mit evaluativen Instrumenten wie Berichtspflichten und Prüf- oder Klagerechten erfolgen.

Mit der Reichweite eines Instruments ist der Umfang seines Geltungsbereichs gemeint. Die Reichweite kann sich dabei einerseits aus der direkten Definition eines personalen oder zeitlichen Geltungsbereichs ergeben, in der Gleichstellungspolitik etwa durch die Benennung von abgegrenzten Zielgruppen (z.B. alle Frauen, Angestellte, BeamtInnen, bestimmte Besoldungsgruppen). Dabei müssen aber auch noch Spezifikationen mitbetrachtet werden, welche die faktische Geltung mitbestimmen. So kann etwa die Reichweite eines Rechtsanspruchs auf Teilzeitarbeit dadurch eingeschränkt sein, dass etwa die Größe eines Betriebs, die Dauer der Betriebszughörigkeit einer Person oder die Möglichkeit der Geltendmachung „,wichtiger Gründe“ den Rechtsanspruch faktisch einschränken (Koch 2007). So leidet etwa das deutsche Antidiskriminierungsrecht darunter, dass es keine Verbands- oder Sammelklagen ermöglicht wie in den USA oder der Schweiz. Damit muss die

10 Funktionsweise ,freiwilliger“ Regelwerke, etwa des Deutschen Corporate-GovernanceKodexes (Töller 2009). 
unmittelbare Wirkung von Feststellungen einer individuellen Klage eng begrenzt bleiben - die Reichweite der sanktionsfähigen Vorschrift ist sehr gering (Bothfeld et al. 2010).

Die Ausdifferenziertheit eines Instruments schließlich ist ein besonderes Qualitätskriterium, da es der Komplexität der Sachverhalte und der Interaktion verschiedener Bereiche Rechnung trägt. Ausdifferenzierte Instrumente können deswegen effektiv sein, weil die Handlungslogiken der AdressatInnen bekannt sind und mögliche Ausweichreaktionen antizipiert werden. Die Ausdifferenzierung kann dabei durch die Definition und Unterscheidung von AdressatInnen erfolgen, die Festlegung von zeitlichen Fristen zur Messung der Zielerreichung (z.B. bei der Umsetzung von Quotenregelungen) und ganz grundsätzlich durch die Verknüpfung unterschiedlicher Steuerungsformen zur Ausgestaltung des Prozesses der Implementation. Verschiedene Quotenregelungen unterscheiden sich in ihrer Qualität etwa dadurch, dass das Steuerungsziel (z.B. das Erreichen eines bestimmten Frauenanteils in Führungspositionen) mit Umsetzungsfristen, Planerstellungs- und Berichtspflichten sowie Kontrollmechanismen verknüpft sein kann (siehe Bothfeld/Rouault 2015). Diese Diskussion zeigt aber auch, dass eine einzelne sanktionsbewehrte Vorschrift nicht an sich effektiv ist, sondern dass sie dafür gut mit anderen Steuerungsinstrumenten verbunden sein muss.

\subsection{Untersuchungsdesign und empirisches Vorgehen}

Ausgehend vom gewählten „most similar systems design“ steht im Zentrum unserer empirischen Analyse der Vergleich der Steuerungsregime in der Gleichstellungspolitik der Bundesverwaltung in den drei Ländern Deutschland, Österreich und der Schweiz. Wie sind deren institutionelle und substanzielle Instrumente jeweils ausgestaltet? Wie ist die Politik koordiniert? Wir vergleichen diese Steuerungsregime im Hinblick auf ihre spezifische Ausgestaltung (Verbindlichkeit, Ausdifferenziertheit, Reichweite) mit einem besonderen Augenmerk auf die Konsistenz und mögliche Spannungsverhältnisse zwischen verschiedenen Maßnahmen. Inwiefern stellen sich die gleichstellungspolitischen Strategien anhand ihres Steuerungsregimes unterschiedlich oder ähnlich dar? Kommen die gleichen Instrumente zur Anwendung? Sind die Instrumente hinsichtlich ihrer Ausgestaltung wirklich vergleichbar? Schließlich explorieren wir, in welcher Weise die Instrumente der Gleichstellungspolitik mit denen der Personalpolitik verknüpft sind: Fügen sich die gleichstellungspolitischen Anforderungen gut in die bestehenden Strukturen ein oder produzieren sie Reibungen und Widersprüchlichkeiten mit den gegebenen institutionellen Strukturen der Personalpolitik? Darauf aufbauend ziehen wir Schlüsse zur jeweiligen Effektivität der Steuerungsregime. 
Die Analysen dieses Buches basieren auf dem Vergleich dreier Fallstudien von Bundesverwaltungen in Staaten mit föderalem Regierungssystem. Somit ist gewährleistet, dass die Aufgabenstellungen der Bundesverwaltungen ähnlich und damit vergleichbar sind. ${ }^{11}$ Auf die dennoch bestehenden unterschiedlichen Beschäftigungsgruppen, die der Bundesverwaltung in den Ländern zugeordnet werden, wird bei den Fallstudien eingegangen. Ähnlichkeit und Vergleichbarkeit zwischen der Schweiz, Österreich und Deutschland ist zudem dadurch gegeben, dass es sich in allen drei Fällen um Wohlfahrtsstaaten der konservativ-korporatistischen Tradition handelt, in denen sich das Geschlechterverhältnis erst in einer Übergangsphase zum Doppelverdienermodell befindet.

Innerhalb der Fallstudien haben wir uns auf kleinere Einheiten, nämlich einzelne Ämter und Ministerien, konzentriert, weil eine Untersuchung der jeweiligen gesamten Bundesverwaltungen nicht möglich war. Die Fallauswahl der Ämter bzw. Ministerien war geleitet durch Fragestellungen des schweizerischen Projekts (siehe Einleitung). Dort wurden die Einheiten anhand zweier wichtiger Faktoren ausgewählt, für die ein Einfluss auf betriebliche Gleichstellungspolitik vermutet werden konnte, nämlich der Anteil von Frauen an Führungspositionen und das Ausmaß der gleichstellungsrelevanten politisch-inhaltlichen Aktivitäten. ${ }^{12}$ So wurden in der Schweiz vier Ämter untersucht, in denen diese Faktoren jeweils unterschiedlich kombiniert sind:

- Direktion für Entwicklung und Zusammenarbeit DEZA - hoher Frauenanteil in Führungspositionen, viele gleichstellungsrelevante Aktivitäten;

- Staatssekretariat für Wirtschaft SECO - niedriger Frauenanteil in Führungspositionen, viele gleichstellungsrelevante Aktivitäten;

- Bundesamt für Landwirtschaft BLW - hoher Frauenanteil in Führungspositionen, wenige gleichstellungsrelevante Aktivitäten;

- Eidgenössische Finanzverwaltung EFV - niedriger Frauenanteil in Führungspositionen, wenige gleichstellungsrelevante Aktivitäten.

In der Schweiz haben die Bundesämter ein großes Maß an Unabhängigkeit unter dem Dach eines Departements, welches meist nur eine kleine zentrale

11 Es kamen nur solche Verwaltungseinheiten in Betracht, die erstens genuin politische und nicht lediglich administrative Aufgaben haben und die zweitens eine Mindestgröße von etwa 50 Personen. Zudem müssen deren Aufgaben in allen drei Ländern auf Bundesebene in der unmittelbaren Verwaltung erbracht werden (und nicht etwa an Agenturen ausgelagert sein).

12 Dies erfolgte durch Daten des Eidgenössischen Personalamts und durch eine Webrecherche bei in Frage kommenden Ämtern nach „Gleichstellung“" und „Chancengleichheit“. Zusätzlich wurden Aussagen einer Auftragsstudie herangezogen, die zu Kooperationen und Gleichstellungsprojekten innerhalb der Bundesverwaltung gemacht wurden (Fuchs 2011). Wie in der Analyse zu Vorstellungen von Geschlecht und Gleichstellung im vierten Kapitel dieses Buchs ausgeführt wird, prägt eine umfassende langjährige inhaltliche Arbeit für die Gleichstellung die Wahrnehmungen der Beschäftigungen stark mit, während die Durchführung nur einzelner Projekte keinen Einfluss hat. 
Einheit besitzt. Dabei vergleichen wir schweizerische Ämter als funktionale Äquivalente zu den Ministerien in den beiden anderen Ländern. In Deutschland und Österreich wurden angesichts begrenzter Projektressourcen jeweils zwei Ministerien untersucht. Dabei wählten wir die jeweiligen Finanz- und Arbeitsministerien als Pendants zur schweizerischen Finanzverwaltung bzw. des Staatssekretariat für Wirtschaft aus. Dies ermöglichte uns, einerseits in jedem Land die gleichen Ministerien zu untersuchen, andererseits innerhalb der Länder möglichst kontrastreiche Beispiele in Bezug auf Präsenz von Frauen in Führungspositionen und gleichstellungsrelevante Tätigkeiten zu erfassen.

Wir haben unsere Daten für die konkreten Fallstudien in aufeinanderfolgenden Schritten erhoben. Zuerst analysierten wir die rechtlichen Rahmenbedingungen anhand von Rechtsquellen und Gesetzeskommentaren. Danach analysierten wir Politikdokumente wie Leitfäden, Gleichstellungs- und Personalentwicklungspläne sowie öffentliche und interne Informationsmaterialien. Dazu gehörten Statistiken und - soweit vorhanden und zugänglich regelmäßige Reportings und Erfahrungsberichte. Daran schlossen sich zuletzt ExpertInneninterviews an, aus denen Informationen zur Funktionsweise und Nutzung konkreter Instrumente gewonnen wurden. Aufgrund der politischen Exponiertheit war der Feldzugang voraussetzungsvoll: Interviews mit relevanten AkteurInnen mussten teilweise von den Leitungen erfragt und genehmigt werden, zudem erschwerten beispielsweise in Deutschland personelle Wechsel im für Gleichstellungspolitik zuständigen Ressort des Bundesfamilienministeriums die Identifikation relevanter GesprächspartnerInnen. Dort verhinderte die große tagespolitische Aufmerksamkeit für das Gleichstellungsthema die Möglichkeit kurzfristiger Interviewtermine. Der Zugang erfolgte in allen drei Verwaltungen über Schlüsselpersonen vornehmlich in den jeweiligen Personalabteilungen. Diese schlugen auch Gesprächspersonen vor und waren teilweise bei der Terminvereinbarung behilflich. Mit einem gemeinsam entwickelten und auf die jeweiligen Verwaltungen angepassten Interviewleitfaden wurden pro Land zwischen sieben und 28 qualitative ExpertInneninterviews mit Gleichstellungsbeauftragten, Vorgesetzten, Leitungspersonen und Personalfachleuten geführt, um möglichst unterschiedliche Perspektiven auf das Instrumentarium der Gleichstellungspolitik und seine Funktionsweise in der Praxis zu bekommen (vgl. Bogner et al. 2005). Hinzu kamen Hintergrundgespräche mit externen ExpertInnen und GewerkschaftsvertreterInnen. Die Interviews wurden selektiv transkribiert und inhaltsanalytisch ausgewertet. Für den Vergleich wurden die Fallstudien nach einem gemeinsamen Bewertungsraster analysiert und dargestellt. Insgesamt ist unser Vorgehen qualitativ orientiert, wir nutzen aber verschiedenartige empirische Quellen, um Phänomene und Entwicklungen auch zu quantifizieren. Tafel 2.1 gibt einen Überblick über die Untersuchungsgegenstände und das empirische Vorgehen. 
Tafel 2.1: Untersuchte Verwaltungseinheiten und Analyseschritte

\begin{tabular}{|c|c|c|c|c|}
\hline & chweiz & \multicolumn{2}{|l|}{ Deutschland } & sterreich \\
\hline 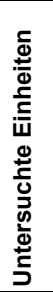 & $\begin{array}{l}\text { Direktion für Entwicklung } \\
\text { und Zusammenarbeit } \\
\text { DEZA } \\
\text { Staatssekretariat für } \\
\text { Wirtschaft SECO } \\
\text { Bundesamt für Landwirt- } \\
\text { schaft BLW } \\
\text { Eidgenössische Finanz- } \\
\text { verwaltung EFV }\end{array}$ & \multicolumn{2}{|c|}{$\begin{array}{l}\text { - Bundesministerium für } \\
\text { Arbeit und Soziales } \\
\text { - Bundesministerium der } \\
\text { Finanzen }\end{array}$} & $\begin{array}{l}\text { um für } \\
\text { und } \\
\text { hutz } \\
\text { um für }\end{array}$ \\
\hline 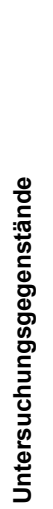 & \multicolumn{2}{|c|}{$\begin{array}{l}\text { Institutionelle Politik } \\
\text { - Gleichstellungsgesetze für die Bundesver- } \\
\text { waltung } \\
\text { - Rechte und Pflichten in der Personalpolitik } \\
\text { (z.B. grundlegendes Fördergebot der } \\
\text { Gleichstellung) } \\
\text { - Gleichstellungspläne } \\
\text { - Kompetenzen, hierarchische Einordnung } \\
\text { und finanzielle wie personelle Ausstattung } \\
\text { der Gleichstellungs- bzw. Gleichbehand- } \\
\text { lungsbeauftragten } \\
\text { - Ihre inhaltlichen Schwerpunktsetzungen in } \\
\text { der Praxis } \\
\text { - Monitoring, Controlling und Evaluation der } \\
\text { Maßnahmen } \\
\text { - Eventuelle Vernetzungsgremien }\end{array}$} & \multicolumn{2}{|c|}{$\begin{array}{l}\text { Substanzielle Politik (vorgesehene und } \\
\text { empirisch vorkommende Maßnahmen) } \\
\text { - Quotenregelungen } \\
\text { - Regelungen zur Elternkarenz } \\
\text { - Teilzeit- und Telearbeitsregelungen } \\
\text { - Weitere Projekte oder Strukturen zur } \\
\text { besseren Vereinbarkeit Familie/Beruf } \\
\text { - Projekte und Verfahren für Lohn- } \\
\text { gleichheit } \\
\text { - Verfahren für Personalrekrutierung } \\
\text { und -bewertung, Laufbahnplanung } \\
\text { und Karriereförderung für Frauen } \\
\text { - Schutz vor sexueller Belästigung } \\
\text { - Gleichstellungmaßnahmen in der } \\
\text { Weiterbildung }\end{array}$} \\
\hline $\begin{array}{l}\text { D. } \\
\text { D }\end{array}$ & \multicolumn{4}{|c|}{$\begin{array}{l}\text { 1. Analyse rechtlicher Rahmenbedingungen } \\
\text { Quellen: Gesetze, Verordnungen, Kommentare } \\
\text { 2. Analyse von Politik-Dokumenten } \\
\text { Gleichstellungspläne, Berichte, Leitfäden, Informationsmaterialien; Personalstatistiken und } \\
\text {-berichte } \\
\text { 3. Leitfadengestützte Expertlnneninterviews } \\
\text { Mit Gleichstellungsbeauftragten, Vorgesetzten und Fachleuten; mit externen ExpertInnen } \\
\text { und GewerkschaftsvertreterInnen } \\
\text { 4. Erstellung der jeweiligen Fallstudie } \\
\text { 5. Vergleichende Analyse der Fallstudien }\end{array}$} \\
\hline
\end{tabular}

Quelle: eigene Darstellung 



\title{
3 Die Bundesverwaltungen als Beschäftigungsfeld
}

\author{
Silke Bothfeld, Gesine Fuchs, Andrea Leitner, Sophie Rouault
}

Vor welchem Hintergrund wird betriebliche Gleichstellungspolitik in den Bundesverwaltungen betrieben? Ziel des folgenden Kapitels ist es, den Bundesdienst als Beschäftigungsfeld $\mathrm{zu}$ verorten, die Arbeitsbedingungen $\mathrm{zu}$ charakterisieren und grundlegende Vergleichszahlen zur Situation der Gleichstellung in den Bundesdiensten Deutschlands, Österreichs und der Schweiz zu präsentieren.

Die Tatsache, dass die in diesem Kapitel vorgestellten Daten grundsätzlich geschlechtersegregiert zur Verfügung stehen und die Entwicklung der Frauen- und Männeranteile in den verschiedenen Bereichen und Tätigkeiten der Bundesdienste fortdauernd überprüft werden, ist keineswegs selbstverständlich. Die Entwicklung entsprechender Indikatoren und des Monitorings an sich sowie die Sensibilisierung für Gleichstellungsfragen, denen wir in unseren Interviews begegnet sind, interpretieren wir auch als einen langfristigen Sensibilisierungsfortschritt von Gender-Mainstreaming-Projekten, die in allen drei Ländern Anfang des neuen Jahrtausends begonnen wurden. Mit diesen Projekten sollten Instrumente und Umsetzungsbeispiele entwickelt werden, wie die Gleichstellungsperspektive in die inhaltliche Arbeit aller Politikfelder mit dem Ziel einer Gleichstellung von Frauen und Männern integriert werden kann. Trotz der Investition in Strukturmaßnahmen mit zum Teil erheblichen Ressourcen (etwa durch die mittlerweile eingestellte Finanzierung des Genderkompetenzzentrums an der Humboldt-Universität zu Berlin) wurde die Integration des Gender Mainstreamings in den letzten Jahren in Deutschland nicht weiter verfolgt. ${ }^{1}$ In der Schweiz folgten auf die Pilotprojekte in der Bundesverwaltung keine weiteren Arbeiten. ${ }^{2}$ Auch für

1 Zur Situation in Deutschland vgl. Lewalter et al. (2009). In Deutschland gilt Gender Mainstreaming (GM) weiterhin als verbindliches Leitprinzip in der Gemeinsamen Geschäftsordnung der Bundesministerien, doch der Regierungswechsel 2005 und die Priorisierung von Familienpolitik anstelle von Gleichstellungspolitik haben dazu beigetragen, dass die wissenschaftlichen Begleitstrukturen und die interministeriale Arbeitsgruppe abgeschafft wurden und so GM nicht vollständig in die Arbeitsroutinen integriert werden konnte. Dennoch stellen die beteiligten ForscherInnen fest, dass die Verantwortung zum gleichstellungspolitischen Handeln anerkannt wurde und Gender-Wissen nun zu den anerkannten Fachkompetenzen gehört. In den Chancengleichheitsweisungen für die schweizerische Bundesverwaltung wird GM als Prinzip des gesamten Human-Resource-Managements postuliert.

$2 \mathrm{Zu}$ den Bemühungen in der Schweiz siehe Eidgenössisches Büro für die Gleichstellung von Frau und Mann (2004). Viele Gleichstellungsbeauftragte sehen nach wie vor im GM eine ebenso sinnvolle wie anspruchsvolle Strategie, die jedoch abhängig vom politischen Willen und der Existenz einer fachkompetenten und starken Gleichstellungsbeauftragten sei (Fuchs 2011). 
Österreich ist ein abnehmendes Engagement für Gender Mainstreaming trotz der institutionellen Verankerung u.a. über die Interministerielle Arbeitsgruppe Gender Mainstreaming und Gender Budgeting zu beobachten, bleibt aber durch die Fokussierung der geschlechtergerechten Budgetgestaltung (Gender Budgeting) eine wesentliche Antriebskraft für die Gleichstellungspolitik (vgl. Autorinnenkollektiv 2009; Gstrein et al. 2013). ${ }^{3}$

\subsection{Grundprinzipien und Entwicklungslinien des öffentlichen Dienstes}

Unser Untersuchungsgegenstand, der Bundesdienst in Deutschland, Österreich und der Schweiz, weist als Teil des öffentlichen Dienstes einige charakteristische Gemeinsamkeiten auf. In der vergleichenden Bürokratieforschung werden die Länder einer ,germanischen“ bzw. einer kontinentaleuropäischföderalen Tradition zugeordnet (vgl. Painter/Peters 2010: 20-22; Kuhlmann/ Wollmann 2013: 19-29; Giauque 2013). Sie bilden im Hinblick auf Rechtsund Verwaltungstraditionen und Verwaltungsorganisation eine eigene Gruppe. Dazu gehört die starke Betonung demokratischer Rechtsstaatlichkeit im Verwaltungshandeln, das sich durch Rechtsbindung, legalistische Gestaltung der Normen und besonders in Deutschland durch die Betonung der Grundrechte als Beschränkung staatlicher Machtentfaltung auszeichnet (Grunow 2009: 358f.). Daraus folgt eine starke Stellung des Verwaltungsrechts und von JuristInnen innerhalb des öffentlichen Dienstes. Die Länder sind föderal organisiert und vom Subsidiaritätsprinzip geprägt, was zu starken Kommunalverwaltungen und ,schlanken“ bundesstaatlichen Verwaltungen führt. Es gibt einen ausgeprägten kooperativen Verwaltungs- bzw. Vollzugsföderalismus und eine enge Verflechtung zwischen staatlicher und zivilgesellschaftlicher Aufgabenerfüllung. Generell kennen öffentliche Verwaltungen zwei Kategorien von Beschäftigten, nämlich in der Regel lebenslang beschäftigte Beamte und Angestellte (Temmes 2011: 2157). Für die germanische Tradition ist die Figur des Berufsbeamten besonders prägend, obgleich quantitativ das Angestelltenverhältnis vorherrscht. Der Beamte setzt sich in einer festen Hierarchie eines Verwaltungsstabs mit klaren Kompetenzen uneigennützig für das Allgemeinwohl ein. Dabei regelt sich der Zugang durch beglaubigte Fachqualifikationen (und nicht Wahl). Beamte behandeln ihr Amt als einzigen Beruf und werden in ein unkündbares Amtsverhältnis mit einer Laufbahn übernommen, woraus sich besondere Treuepflichten einerseits und besondere

3 Auf die Umsetzung und Bedeutung von Gender Mainstreaming und Gender Budgeting in der Gleichstellungspolitik des Bundes in Österreich wird im Länderkapitel näher eingegangen. 
Fürsorgepflichten des Dienstherrn andererseits ergeben. ${ }^{4}$ Vor allem die Schweiz weist aber auch liberale und staatskritische Traditionen und Reformen auf. So kennt sie die Volkswahl bestimmter öffentlicher Funktionen beim Gericht oder bei der Schulaufsicht und hat den Beamtenstatus fast überall abgeschafft: Beschäftigte sind eher „Angestellte des Volkes“ als „Staatsdiener".

Die Institution des Beamtentums ist männlich tradiert. In historischer Perspektive ist die Vorstellung des Beamten, der sich ausschließlich und lebenslang dem öffentlichen Gemeinwohl verpflichtet sieht, klar männlich konnotiert (vgl. Gottschall 1990). Frauen als Beamtinnen stehen im Widerspruch zu bürgerlichen Geschlechterideologien und -hierarchien, die Frauen eine Rolle als Hausfrau und Mutter zuwies. Familienpflichten wurden als unvereinbar mit Dienstpflichten und ungeteilter Loyalität angesehen: So wurde das Beamtinnenzölibat, das bei Heirat eine Entlassung von Frauen aus dem Staatsdienst vorsah, in Deutschland erst 1953 endgültig abgeschafft (vgl. Kling 1999; von Oertzen 1999b), in Österreich 1920; in der Schweiz hielten sich solche Regeln, kantonal unterschiedlich, bis in die 1960er Jahre. ${ }^{5}$ Auch wurde die Möglichkeit der Teilzeitarbeit für Beamtinnen erst spät eingeführt (Deutschland 1969, Österreich 1979, Schweiz 1987) (Ministerium für Generationen 2008: 73; Keller 2012; BKA 2012). Höhere Frauenanteile im Bundesdienst föderaler Staaten fallen umso mehr ins Gewicht und können umso stärkere Signalwirkungen entfalten, als dieser Dienst mit seinem substanziellen Anteil an hoheitsrechtlichen Aufgaben stärker männlich konnotiert ist als etwa kommunale Dienste mit ihren typischen Aufgaben im Sozial- und Gesundheitsbereich (vgl. Gottschall 2009).

Eine wesentliche Entwicklungstendenz ist die Stagnation bzw. der Rückgang der öffentlichen Beschäftigung seit Ende der 1980er Jahre. Regierungen mit tendenziell neoliberaler Programmatik traten für ,,mehr Markt“, reduzierte öffentliche Leistungen, den Abbau öffentlicher Beschäftigung und seine „De-Privilegierung“ ein. Dabei wurden zum Teil Aufgaben an Agenturen oder öffentlich-rechtliche Betriebe ausgelagert, etwa im Bereich Gesundheit oder Versorgung, oder es wurden durch Personalabbau effektiv Kosten eingespart (Reichard/Schröter 2009: 23). Die Verhandlungsmacht der Gewerkschaften hat dabei ebenso abgenommen wie die diffuse politische Unterstützung der weiteren Öffentlichkeit.

Während New Public Management (NPM), also die Übernahme privatwirtschaftlicher Managementtechniken in die öffentliche Verwaltung (vgl. Savoie 2011) schon in den 1990er Jahren Einzug hielt, folgte eine Veränderung der entsprechenden Personalpolitik erst später. NPM sieht die Mitarbei-

4 Vgl. Grunow (2009: 366) mit Verweis auf den Weber'schen Idealtypus legaler Herrschaft durch einen bürokratischen Verwaltungsstab.

5 Zum Beispiel Abschaffung im Kanton Zürich für Lehrerinnen 1962; vgl. de.wikipedia.org/ wiki/Lehrerinnenz\%C3\%B6libat (Zugriff: 15.09.2014). 
tenden als einen strategischen Erfolgsfaktor zum Erreichen langfristiger Verwaltungsziele und postuliert folgerichtig ein strategisches Human Resource Management, die enge Verknüpfung und Abstimmung von Personalstrategie mit allgemeiner Organisationsstrategie, die Kohärenz der Personalarbeit über alle Hierarchieebenen hinweg und die Dezentralisierung der Personalarbeit auf untergeordneten Organisationsebenen (Thom/Alfes 2007). Im Zuge dessen sind die Laufbahnstrukturen des öffentlichen Dienstes offener, flexibler und mitunter sogar prekär geworden. ${ }^{6}$ Personalpolitische Entscheidungskompetenzen wurden vielfach dezentralisiert und Leistungselemente in der Bezahlung gestärkt (vgl. dazu Demmke 2011: isb. 77-79). Insgesamt setzen sich also marktorientierte Koordinierungs- und Steuerungsprinzipien stärker in den öffentlichen Beschäftigungssystemen durch, und zwar zu Lasten der tradierten hierarchie- und regelbetonten Gemeinschafts- und Teilhabeorientierung (Reichard/Schröter 2009: 25). Aus der NPM-Perspektive wirkt sich eine größere Diversität im öffentlichen Dienst - und damit auch eine ausgeglichenere Geschlechterverteilung - vor allem positiv im Hinblick auf Servicequalität, Effektivität und Effizienz aus; gesellschaftspolitische Aspekte wie mehr Geschlechtergerechtigkeit spielen eine untergeordnete Rolle (vgl. OECD 2011b: Kap. 4). Im Gegensatz zur Privatwirtschaft haben öffentliche Verwaltungen einen verfassungsrechtlichen Auftrag zur Förderung der Gleichstellung von Frauen und Männern, und dieser bezieht sich auch auf das eigene Personal. Dabei sind die rechtlichen Vorgaben unterschiedlich und reichen von einer Zielbestimmung in der Rahmengesetzgebung des schweizerischen Bundespersonalgesetzes bis hin zu detaillierteren prozeduralen Vorgaben etwa im deutschen Bundesgleichstellungsgesetz.

\subsection{Rechtsgrundlagen der Gleichstellung im Bundesdienst}

In allen drei Ländern prägt zunächst das nach und nach modernisierte Personalrecht die Behandlung der Beschäftigten im Bundesdienst. Es sieht spezifische Regelungen für die Bundesbediensteten im Hinblick auf die Vereinbarkeit von Familie und Beruf vor und enthält die allgemeine Zielstellung der Gleichbehandlung. In Deutschland und Österreich gelten die allgemeinen arbeitsrechtlichen Regelungen (Elternzeit, Recht auf Teilzeitarbeit, Pflegezeit) auch für die BeamtInnen, und es besteht ein Benachteiligungsverbot bei Einstellung und Beförderung. In der Schweiz sind im Personalrecht hingegen konkrete Gleichstellungsziele (Vereinbarkeit, Frauen in Führungspositionen)

6 So verbeamtet das Land Berlin seine Lehrkräfte seit 2002 nicht mehr, darüber hinaus bezahlt es aus anderen Bundesländern mit Mangelfächern angeworbene Lehrkräfte zum Teil besser, und Vertretungslehrkräfte werden nur befristet unter Ausschluss der Sommerferien beschäftigt. 
verankert, sodass dies über das schweizerische Gleichstellungsgesetz hinausgeht. Es eröffnet den Departementen und Ämtern somit großen Handlungsspielraum für die Herstellung von Geschlechtergleichheit, während es nur wenige im Gleichstellungsgesetz niedergelegte Pflichten für die Privatwirtschaft gibt.

In Österreich und Deutschland und in geringerem Maße in der Schweiz wird die gesetzliche Basis für die konkrete Ausgestaltung der Gleichstellungspolitik im Bundesdienst durch die allgemeinen und spezifischen Gleichstellungs- bzw. Gleichbehandlungsgesetze bzw. -verordnungen ergänzt, die in allen Ländern in den 1990er Jahren eingeführt wurden (Tafel 3.1).

Tafel 3.1: Rechtlicher Rahmen für die Gleichstellung im Bundesdienst

\begin{tabular}{|c|c|c|c|}
\hline & Deutschland & Österreich & Schweiz \\
\hline Personalrecht & $\begin{array}{l}\text { Bundesbeamtengesetz } \\
(1953 / 2009) \text { : } \\
\text { Beurlaubung, Mutter- } \\
\text { schutz, Elternzeit, } \\
\text { Pflegezeit, Teilzeit }\end{array}$ & $\begin{array}{l}\text { Beamtendienstrechts- } \\
\text { gesetz (1979) und } \\
\text { Vertragsbediensteten- } \\
\text { gesetz (1948): } \\
\text { Karenz, Teilzeit, } \\
\text { Telearbeit }\end{array}$ & $\begin{array}{l}\text { Bundespersonalgesetz } \\
\text { (2002): } \\
\text { Fördergebot Gleich- } \\
\text { stellung, Vereinbarkeit }\end{array}$ \\
\hline $\begin{array}{l}\text { Allgemeines } \\
\text { Gleichstellungs- } \\
\text { recht }\end{array}$ & $\begin{array}{l}\text { Allgemeines Gleichbe- } \\
\text { handlungsgesetz } \\
\text { (2006): Basis für } \\
\text { individuelle Klagen } \\
\text { gegen Diskriminierung } \\
\text { aller Art }\end{array}$ & $\begin{array}{l}\text { Gleichbehandlungsge- } \\
\text { setz (2004; } 1979 \text { für } \\
\text { Entgelt) }\end{array}$ & $\begin{array}{l}\text { Gleichstellungsgesetz } \\
\text { (1996) }\end{array}$ \\
\hline $\begin{array}{l}\text { Gleichstellungsge- } \\
\text { setz für den Bun- } \\
\text { desdienst }\end{array}$ & $\begin{array}{l}\text { Frauenfördergesetz } \\
\text { (für die Bundesverwal- } \\
\text { tung 1994) } \\
\text { Bundesgleichstel- } \\
\text { lungsgesetz (BGleiG } \\
\text { 2001: erweitert die } \\
\text { Rechte der GBA, führt } \\
\text { die Quotierung ein) }\end{array}$ & $\begin{array}{l}\text { Bundesgleichbehand- } \\
\text { lungsgesetz (1993; } \\
2004 \text { Erweiterung auf } \\
\text { zusätzliche Diskrimi- } \\
\text { nierungsgründe, 2011 } \\
\text { Einkommensbericht) }\end{array}$ & $\begin{array}{l}\text { Weisungen (1991, } \\
2003 \text { ) für den Bundes- } \\
\text { dienst }\end{array}$ \\
\hline $\begin{array}{l}\text { Exklusiv Bundes- } \\
\text { dienst }\end{array}$ & $\mathrm{Ja}$ & $\mathrm{Ja}$ & Nein \\
\hline $\begin{array}{l}\text { Gleichstellungs- } \\
\text { ansatz }\end{array}$ & Frauenförderung & $\begin{array}{l}\text { Gleichbehandlung und } \\
\text { Frauenförderung }\end{array}$ & $\begin{array}{l}\text { Allgemeine Antidiskri- } \\
\text { minierung }\end{array}$ \\
\hline
\end{tabular}

Quelle: eigene Zusammenstellung

Diese gesetzlichen Grundlagen basieren einerseits auf dem internationalen Abkommen zur Beseitigung jeder Form von Diskriminierung (CEDAW) sowie für Österreich und Deutschland zudem auf dem Rechtsrahmen der Europäischen Union (Rosenberger 2009; Kodré/Müller 2003). Diese Gleichstellungsgesetze können jedoch, wie etwa bei den Vorschriften zur Aufstiegsförderung oder Entlohnung, mit den allgemeinen Vorschriften des Personal- 
rechts in Konflikt geraten (z.B. bei der Behandlung von Teilzeitarbeit bei der Beurteilung oder Erfassung von Seniorität).

Das schweizerische Gleichstellungsgesetz sieht hingegen einheitliche Regeln für private und öffentliche Arbeitgeber gleichermaßen vor. Die wichtigste Unterscheidung zwischen öffentlichen und privaten Arbeitgebern liegt darin, dass getrennte Schlichtungsstellen vorgesehen sind, die bei einer vorgerichtlichen Einigung im Rahmen des individuellen Diskriminierungsschutzes helfen sollen. Zudem etabliert es ein Verbandsklagerecht und die Möglichkeit zur Kollektivklage (Fuchs 2013; Bothfeld et al. 2010). Insgesamt regelt das schweizerische Gleichstellungsgesetz aber weniger Gegenstände. Aufgrund dieser sehr unterschiedlichen Grundstruktur ist zu erwarten, dass gleichstellungspolitische Maßnahmen in der schweizerischen Bundesverwaltung sehr viel enger mit der allgemeinen Personalpolitik verflochten sind und es insgesamt $\mathrm{zu}$ weniger Konflikten zwischen den Regelungsbereichen kommt. Andererseits ist zu erwarten, dass sich die deutsche und österreichische Gleichstellungspolitik weiter ausdifferenziert darstellen und die $\mathrm{Zu}-$ sammenarbeit zwischen gleichstellungspolitischen und personalpolitischen Akteuren innerhalb der Ämter voraussetzungsvoll ist.

\section{3 Öffentlich-rechtliche Dienstverhältnisse}

Deutschland und Österreich kennen grundsätzlich zwei Arten von öffentlichen Dienstverhältnissen, nämlich ein Dienstverhältnis als Beamter oder Beamtin oder als Angestellte mit einem Arbeitsvertrag. BeamtInnen sind grundsätzlich unkündbar und haben in der Regel (lebens-)lange Karriereverläufe im öffentlichen Dienst. Bezahlung und Alterssicherung bilden ein eigenes System unabhängig von den allgemeinen sozialen Sicherungssystemen. BeamtInnen dürfen nicht streiken. In Deutschland wird von ihnen ein besonderes Treueverhältnis zur Verfassung gefordert, und sie müssen jederzeit für die freiheitlich-demokratische Grundordnung eintreten. Die Arbeitsbedingungen von BeamtInnen und Angestellten sind unterschiedlich gesetzlich geregelt. Tafel 3.2 fasst die Unterschiede zusammen.

In der schweizerischen Bundesverwaltung wurde hingegen der Beamtenstatus (und das Streikverbot) mit dem neuen Bundespersonalrecht 2002 abgeschafft. Als neuer Standard wurde das öffentlich-rechtliche Arbeitsverhältnis gesetzt, womit explizit die personalwirtschaftliche Flexibilität erhöht werden sollte. In der Botschaft zum neuen Gesetz heißt es eingangs:

„Im globalen Wettbewerb zählen Effizienz und Effektivität des öffentlichen Sektors zu den wesentlichen Standortvorteilen. Angesichts knapper Ressourcen müssen Verwaltungen mittels flexibler Strukturen zu zielführenden Prozessen fähig sein. (...) Will [der Bund] sich dabei weiterhin als attraktiver, konkurrenzfähiger und sozialer Arbeitgeber positionieren, 
Tafel 3.2: Unterschiedliche Statusprinzipien

\begin{tabular}{lll}
\hline & Beamte & Angestellte \\
\hline Unkündbarkeit & Ja & Nein \\
\hline Versorgung & $\begin{array}{l}\text { Alimentation, Pension (Staats- } \\
\text { haushalt) }\end{array}$ & Gehalt, Rente (Versicherung) \\
\hline Streikrecht & Nein & Ja \\
\hline $\begin{array}{l}\text { Besondere Treue- } \\
\text { pflicht }\end{array}$ & Ja & Nein \\
\hline $\begin{array}{l}\text { Regelung der } \\
\text { Arbeitsverhältnisse }\end{array}$ & $\begin{array}{l}\text { Gesetze und Verordnungen (auch } \\
\text { Schweiz); Disziplinarrecht }\end{array}$ & $\begin{array}{l}\text { Tarifvertrag und allgemeines } \\
\text { Arbeitsrecht (Deutschland); Ge- } \\
\text { setze (Österreich) }\end{array}$ \\
\hline Rekrutierung für & $\begin{array}{l}\text { Laufbahn: Die Beamtlnnen durch- } \\
\text { laufen stufenweise bestimmte } \\
\text { Stationen und Funktionen und } \\
\text { steigen in einem bestimmten } \\
\text { Schema auf. } \\
\text { Kaum Quereinstiege, zum Teil } \\
\text { Wechsel zwischen Bundesländern } \\
\text { oder Ministerien schwierig. }\end{array}$ & $\begin{array}{l}\text { Postionstellung für eine } \\
\text { bechsel zwischen öffentlichem } \\
\text { und privatem Sektor möglich und } \\
\text { häufig. }\end{array}$ \\
& & \\
\hline
\end{tabular}

Quelle: in Anlehnung an Bogumil/Jann (2009: 113); Reichard/Schröter (2009: 20-22); Kuhlmann/Wollmann (2013: 37-39) sowie an die Länderberichte

braucht er ein modernes und dynamisches Personalmanagement. Dieses verfolgt drei Hauptziele: Es muss Veränderungsprozesse in der Verwaltung und in den Unternehmungen unterstützen, wirtschaftliche Arbeitsweisen ermöglichen und eine lernfördernde Kultur begünstigen.“" (Bundesrat 1998, S 1598)

Die schweizerische Verwaltung ist wesentlich durchlässiger für Wechsel aus der Privatwirtschaft als die anderer Länder (Hablützel 2013: 93). Auch vor 2002 kannte sie keine klassische lebenslange Karrierelaufbahn von BeamtInnen im Sinne Webers (Giauque 2013: 47f.; Varone 2013: 122). Die schweizerische Bundesverwaltung ist ein Beispiel für das Positionssystem; einige Personalverantwortliche lehnten in den Interviews einen Pool für Führungspositionen teilweise explizit ab, um nicht Hoffnungen auf eine stete Laufbahn zu wecken; andere verwiesen auf Probleme, qualifiziertes erfahrenes Personal zu halten, weil es in den relativ flachen Hierarchien keine (gehaltsmäßigen) Aufstiegsmöglichkeiten gibt.

In Deutschland und Österreich geht der Anteil der BeamtInnen an den Beschäftigten des Bundes langsam zurück. Damit einhergehend wurden auch Unterschiede z.B. bei der Entlohnung (in Deutschland durch TVöD) oder im Pensionsrecht abgebaut. In Österreich sind trotz langsam steigender Flexibilität die lang andauernden und lebenslangen Karriereverläufe innerhalb des öffentlichen Dienstes noch immer sehr häufig (Taubner/Hirsch 2013). Positionen in höheren Führungsfunktionen müssen öffentlich ausgeschrieben und der Auswahlvorschlag einer unabhängigen Begutachtungskommission vorge- 
legt werden. Spitzenfunktionen in der öffentlichen Verwaltung, etwa die Leitung einer Ministerialsektion, werden nur mehr befristet auf fünf Jahre vergeben. Ebenso wird auf internationale Erfahrung Wert gelegt, um eine Karriere in europäischen und internationalen Institutionen zu ermöglichen.

Die Beschäftigungssicherheit liegt im öffentlichen Dienst nach wie vor wesentlich höher als in der Privatwirtschaft; die Schweiz kennt dort nur einen sehr geringen Kündigungsschutz. In Deutschland können BeamtInnen und Beschäftigte mit einem unbefristeten Arbeitsvertrag nicht aus betrieblichen Gründen entlassen werden; in der Schweiz und Österreich müssen ihnen in diesem Fall andere Arbeitsplätze oder Abfindungen angeboten werden (vgl. OECD 2011a: 109). Die Personalverantwortung liegt bei den einzelnen Ministerien, in der Schweiz bei den einzelnen Ämtern; die personalpolitischen Kompetenzen wurden hier von der Legislative auf die Exekutive verschoben, während in Deutschland nach wie vor das Parlament für den Stellenplan verantwortlich ist. Aufgaben des Personalmanagements sind beim österreichischen Bundeskanzleramt, dem deutschen Bundesverwaltungsamt sowie beim Eidgenössischen Personalamt angesiedelt (vgl. dazu auch die Länderkapitel). Auch bei anderen Merkmalen zur Arbeitsplatzqualität wie Mitbestimmung, Arbeitsplatzsicherheit, Vereinbarkeit, Einkommen oder Weiterbildung schneidet der öffentliche Dienst trotz Privatisierungs- und Ökonomisierungstendenzen nach wie vor besser ab als die Privatwirtschaft (vgl. für Deutschland Ellgut/Kohaut 2011).

Im öffentlichen Dienst besteht ein ausdrückliches Gebot, die beruflichen Karrieren von Frauen zu fördern. ${ }^{7}$ Aus gleichstellungspolitischer Sicht fällt auf, dass die Frauenanteile unter den BeamtInnen wesentlich geringer ist als unter den Angestellten: In Deutschland waren 2010 25,9\% der BeamtInnen und RichterInnen Frauen, aber 44,3\% der Tarifbeschäftigten (BMI 2011: 17). In Österreich waren $201257 \%$ aller Bundesbediensteten verbeamtet, doch in allen Aufgabenbereichen waren anteilsmäßig weniger Frauen als Männer in diesem Status - im Verwaltungsdienst beispielsweise 58,3\% der Männer und nur 33,1\% der Frauen (BKA 2012: 39, 32). Festzuhalten bleibt, dass das Anwachsen des Frauenanteils unter den BeamtInnen und die Flexibilisierung öffentlicher Beschäftigung sowie die Erodierung von Beamtenprivilegien gleichzeitig auftreten. Aus gleichstellungspolitischer Sicht sollte die Entwicklung des Beschäftigungsprofils unter den Beamtinnen - eingedenk der männlichen Prägung des Berufsbeamtentums - genauer analysiert werden: In welchen Bereichen arbeiten Frauen mit welchem Beschäftigungsgrad? Gibt es Tendenzen zur Feminisierung einzelner Bereiche und werden „Männerbastionen“" (z.B. Polizei oder Militär) abgebaut? So kann die Entwicklung der Gleichstellung genauer beurteilt werden.

7 In Österreich im Bundes-Gleichbehandlungsgesetz, in der Schweiz im Bundespersonalgesetz und in Deutschland im Bundesgleichstellungsgesetz. 


\subsection{Beschäftigte im Bundesdienst}

In der Schweiz entfallen knapp 10\% der Beschäftigung auf die öffentliche Verwaltung, die Werte sind in Deutschland und Österreich nur wenig höher. Dies liegt wesentlich unter dem OECD-Schnitt von 15\% und auch unter der öffentlichen Beschäftigung liberaler Staaten wie den USA (15\%) oder Großbritannien (18\%; vgl. OECD 2013a: 103). Die Beschäftigung sank zwischen 2001 und 2011 in allen Ländern leicht, am deutlichsten in Österreich mit 1,3 Prozentpunkten.

Tab. 3.1: Anteil der Beschäftigung in der öffentlichen Verwaltung an der Gesamtbeschäftigung $(1995,2001$ und 2011)

\begin{tabular}{lccc}
\hline & 1995 & 2001 & 2011 \\
\hline Deutschland & $12,2 \%$ & $10,9 \%$ & $10,6 \%$ \\
Österreich & $11,8 \%$ & $12,0 \%$ & $10,7 \%$ \\
Schweiz & $7,2 \%$ & $9,9 \%$ & $9,7 \%$ \\
OECD 32 - Durchschnitt & n.a. & $15,9 \%$ & $15,5 \%$ \\
\hline
\end{tabular}

Quelle: OECD (2013a: 102, 2009: 69)

Die Frauenanteile im öffentlichen Dienst, auch auf nationalstaatlicher Ebene, sind in den letzten zehn Jahren in der OECD-Welt gewachsen und liegen durchschnittlich über der weiblichen Beschäftigungsquote in der Gesamtwirtschaft (OECD 2013a: 120-123).

Der Bundesdienst erfasst nur einen kleinen Teil der öffentlichen Beschäftigung aufgrund der föderalen Organisation der öffentlichen Dienste. In Deutschland und der Schweiz entfallen auf den Bundesdienst rund 10\% der öffentlich Bediensteten (OECD 2013a: 105). Österreich vereint dagegen etwa $38 \%$ der öffentlichen Beschäftigung auf den Bundesdienst. ${ }^{9}$ Dies hängt wesentlich mit der Verteilung der Beschäftigten zwischen Ländern und Bund zusammen, indem in Österreich aufgrund von Größe und Organisationsstruktur weniger Aufgaben an die Länder delegiert werden. Die Geschlechterverteilung im Bundesdienst variiert zwischen den Vergleichsländern erheblich: In Deutschland ist nur ein Viertel der Beschäftigten Frauen, in der Schweiz knapp ein Drittel, in Österreich sind dies zwei Fünftel. Vergleicht man hingegen den Frauenanteil nach Tätigkeitsfeldern, sind die Unterschiede zwischen den Ländern deutlich geringer, unterscheiden sich aber wesentlich nach

8 Die Definition des öffentlichen Dienstes folgt dem System of National Accounts (SNA) und beinhaltet neben Gebietskörperschaften (für Bund, Länder, und Gemeinden) auch Institutionen, die unter staatlichem Einfluss stehen und in ihrer Leistungserstellung und Preissetzung nicht marktbestimmt sind, sowie ihre Kosten zu weniger als 50\% aus eigenen Umsätzen decken können (ebd.)

9 Vgl. https://www.oeffentlicherdienst.gv.at/fakten/oesterreich/basiskenndaten/basiskenndaten. html (Zugriff: 19.02.2014). 
den Aufgabenbereichen: Im Bereich der Verteidigung und öffentlichen Sicherheit sind die Beschäftigten stark männerdominiert, der Verwaltungsdienst und die Rechtspflege (Staatsanwaltschaft und Gerichte) ${ }^{10}$ weisen hingegen eine ausgeglichenere Geschlechterverteilung auf. Lehrkräfte, die nur in Österreich auch im Bundesdienst beschäftigt sind, weisen den höchsten Frauenanteil auf.

Abb. 3.1: Frauenanteile im Bundesdienst nach Aufgabenbereichen $(2011 / 2012$, in \%)

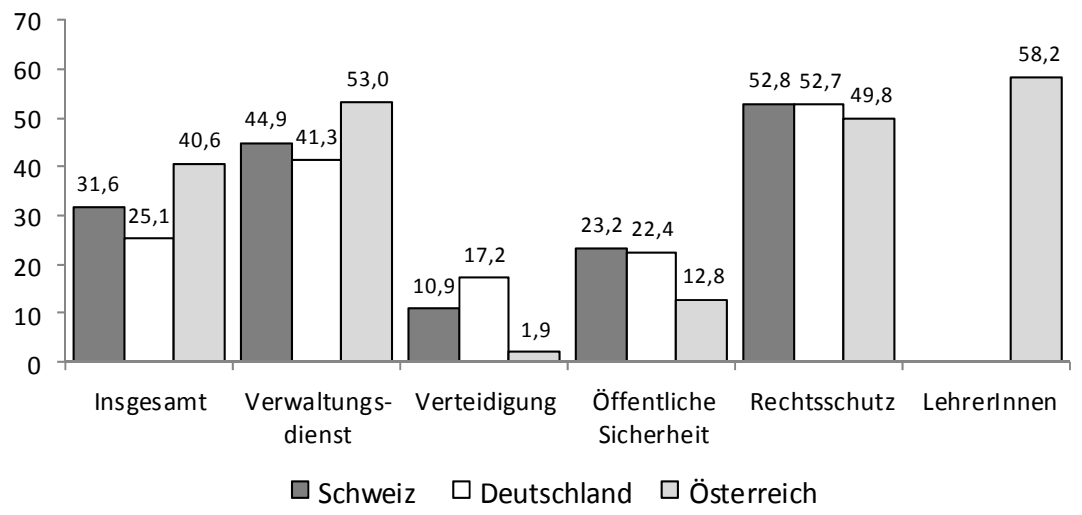

Quelle: Schweiz: EPA-Datensatz (2012), eigene Berechnungen; Österreich: BKA (2012); Deutschland: Destatis (2012)

Damit wird deutlich, dass der Frauenanteil im Bundesdienst eng mit der Struktur des Bundesdienstes verknüpft ist. Der eigentliche Verwaltungsdienst umfasst in Deutschland nur knapp 29\%, in Österreich knapp 35\% und in der Schweiz knapp 55\% der Beschäftigten des Bundesdienstes. Hingegen ist der Anteil von SoldatInnen und weiteren Beschäftigten in der Verteidigung in Deutschland höher (59\% aller Bundesbeschäftigten). In Österreich sind knapp 30\% der Bundesbeschäftigten Lehrkräfte.

Die Zahlen zeigen folgende gemeinsame Charakteristika: Die Rechtspflege ist ein Bereich mit fast ausgeglichenem Geschlechterverhältnis und einer Feminisierung in den letzten Jahren; in den Gesamt-Bundesdiensten ist der Anstieg der weiblichen Beschäftigung moderat, aber stetig: In der Schweiz ist ein Plus von 3,2 Prozentpunkten zwischen 2002 und 2011 auszumachen, für Deutschland 2002 bis 2012 ein Plus von 2,7 Prozentpunkten und in Österreich zwischen 2001 und 2011 um 3,3 Prozentpunkte gestiegen. Das heißt, die Entwicklung des Frauenanteils verläuft in den Vergleichsländern durchaus ähnlich, doch ist das Ausgangsniveau unterschiedlich.

10 In Österreich sind nur RichterInnen erfasst. Die Zahlen aus der Schweiz wurden direkt vom Bundesgericht geliefert. 
Der Anstieg der Frauenbeschäftigung im Bundesdienst geht mit der Erhöhung der Teilzeitarbeit einher. ${ }^{11}$ Zwischen einem Drittel und der Hälfte der weiblichen Beschäftigten in der Gesamtwirtschaft sind teilzeitbeschäftigt, während die Teilzeitquote ihrer männlichen Kollegen die 10\%-Marke nicht überschreitet. Interessanterweise ist Teilzeitarbeit in den Ministerien mit hohem Männeranteil auch unter den dort beschäftigten Frauen weniger verbreitet als in Ressorts mit höherem Frauenanteil.

Abb. 3.2: Teilzeitarbeit im Bundesdienst und in der Gesamtwirtschaft nach Geschlecht (2011, in \%)

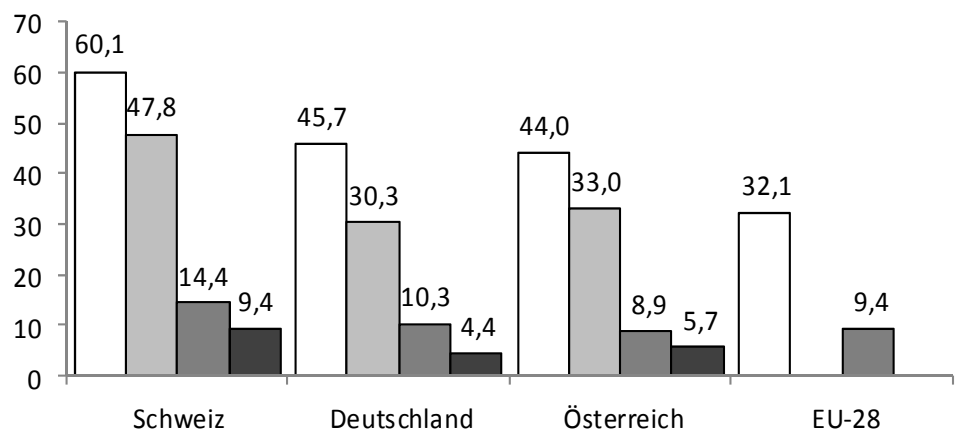

$\square$ TZ-Quote Gesamtwirtschaft Frauen $\square$ TZ-Quote Bundesdienst Frauen

$\square$ TZ-Quote Gesamtwirtschaft Männer $\square$ TZ-Quote Bundesdienst Männer

Quelle: Bundesdienst: Schweiz: EPA-Datensatz (2012); Deutschland: Destatis (2012); Österreich: BKA (2012); Gesamtwirtschaft: http://epp.eurostat.ec.europa.eu/tgm/ table.do?tab=table\&init $=1$ \&plugin $=1$ \&language $=$ en $\&$ pcode $=$ tps00159 $\quad$ (Zugriff: 21.02.2014).

Im Bundesdienst weist Österreich die niedrigste und die Schweiz die höchste Teilzeitquote auf. Gemeinsam ist den Ländern, dass im Bundesdienst wesentlich weniger als in der Gesamtwirtschaft in Teilzeit gearbeitet wird. Ein Hinweis dafür ist die Tatsache, dass BeamtInnen in Deutschland und Österreich nach wie vor wesentlich öfter Vollzeit arbeiten als die Tarifbeschäftigten. ${ }^{12}$ In der Schweiz konnte aus den Daten errechnet werden, mit welchen Pensen die Beschäftigten arbeiten: Dabei zeigt sich, dass Teilzeitpensen unter 50\% tendenziell abgenommen haben, auch bei Frauen, und dass die „große Teilzeit“

11 In Deutschland verdoppelte sich im Bundesdienst der (niedrige) Anteil der Teilzeitbeschäftigung zwischen 1991 und 2010 von 3,6\% auf 6,9\% (BMI 2011: 19; ohne Altersteilzeit). In der Schweiz stieg er zwischen 2002 und 2011 von $16,2 \%$ auf $19,6 \%$, und in Österreich wuchs der Anteil seit Mitte der 1990er Jahre bis 2011 relativ stark von knapp 10\% auf knapp $17 \%$.

12 In Österreich 7\% vs. 30\% (BKA 2012: 48), in Deutschland 13\% vs. 25\% (BMI 2011: 30f. und 36f.). 
ab 70\% zugenommen hat. ${ }^{13}$ Zwar ist Teilzeitarbeit in niedrigen Lohnklassen am stärksten verbreitet und im Topmanagement quasi inexistent, im mittleren Management nimmt sie aber langsam zu. Damit könnte sich ein Trend zu qualifizierter und existenzsichernder Teilzeitarbeit andeuten. In den Interviews wurde übereinstimmend auch immer wieder diese „große Teilzeit“ aus arbeitstechnischen und kulturellen Gründen klar favorisiert, gerade für qualifizierte Arbeitsplätze, während die kleinen Pensen als tendenziell problematisch bewertet wurden. Festzuhalten bleibt, dass sich in keinem Land trotz Anstieg der Teilzeitarbeit die geschlechtsspezifischen Muster der Erwerbsarbeit verändert haben. Die Unterschiede im Arbeitsvolumen von Erwerbsarbeit und von Haus- und Sorgearbeit bestehen nach wie vor. Ob mehr Teilzeitarbeit dennoch das Potenzial hat, diese Muster aufzubrechen, muss offen bleiben.

\subsection{Entlohnung und Gender Pay Gap}

Das System der Entlohnung und der Regeln zur Einstufung, Beförderung sowie zu Leistungszulagen ist im öffentlichen Dienst rechtlich reguliert. Diese Aspekte sind für Fortschritte in der betrieblichen Gleichstellungspolitik besonders relevant. ${ }^{14}$

In Deutschland orientiert sich die Personalpolitik an vier Laufbahngruppen, welche die Berufswege der Beamtenschaft und das Besoldungssystem strukturieren (einfacher, mittlerer, gehobener und höherer Dienst). Jede Laufbahn setzt einen bestimmten Ausbildungsgang voraus und hat eigene Besoldungsgruppen und Aufstiegsmöglichkeiten. Ein Wechsel zwischen den Laufbahnen ist nur selten möglich. Nach jahrelangen Verhandlungen zwischen Arbeitgebern und Gewerkschaften wurde 2005 der neue Tarifvertrag für den öffentlichen Dienst (TVöD) eingeführt. Wesentliche Neuerungen sind die Vereinheitlichung des Tarifwerks für BeamtInnen und Angestellte und die Ersetzung der dienstalters- und familienbezogenen Bezahlung durch eine erfahrungs- und leistungsorientierte Vergütung (Bogumil/Jann 2009: 112f.). Die Leistungskomponente sollte bis zu einem (nicht festgelegten) Zeitpunkt $8 \%$ der Lohnsumme darstellen - die Reform wurde mit 1\% begonnen.

In Österreich sind die Gehälter der BeamtInnen und der Vertragsbediensteten in unterschiedlichen Gesetzen geregelt, folgen aber ähnlichen Prinzipien und haben dieselben Bestandteile (Entgelt, Zulagen, Nebengebühren, sonstige Zahlungen). Die Feststellung des Entgelts erfolgt sowohl bei BeamtInnen

132011 waren Frauen nur zu 12\% unter 50\% beschäftigt, weitere $17 \%$ unter $70 \%$ (eigene Berechnungen nach EPA-Datensatz).

14 In Deutschland sind auch gültige Tarifverträge unmittelbar geltendes Recht zwischen den Vertragsbeteiligten, vgl. § 4 Tarifvertragsgesetz. 
als auch bei Vertragsbediensteten auf Grundlage ihrer Verwendung, die die Aufgaben der Arbeitsplätze nach einem einheitlichen Schema bewerten. Den Arbeitsplätzen werden Verwendungsgruppen bzw. Bewertungsgruppen zugeordnet, die darüber hinaus in Funktionsgruppen gegliedert werden. Garantierte Gehaltserhöhungen erfolgen nach jeweils zwei Jahren. Erhöhungen können aber durch funktionsabhängige Zulagen für besondere dienstliche Aufgaben erreicht werden oder wenn durch eine Verwendungsänderung eine höhere Entlohnungsgruppe zum Tragen kommt. Für die Erbringung einer besonderen Leistung können zusätzlich Leistungsprämien gezahlt werden, die aufgrund ihrer Höhe aber eher symbolischen Charakter haben. Insgesamt besteht ein relativ starres Besoldungssystem, das aber durch die Bewertung des jeweiligen Arbeitsplatzes, durch Prämien und Zulagen und nicht zuletzt durch unterschiedliche Karrierewege zu unterschiedlichen Einkommensentwicklungen führen kann.

In der Schweiz wird jeder Arbeitsplatz in der Bundesverwaltung bewertet und in eine von 38 Lohnklassen eingeteilt, die mehrere sog. Erfahrungsstufen haben. Die Lohnklasse eines Arbeitsplatzes wird anhand des Pflichtenheftes und Wegleitungen des Eidgenössischen Personalamts bestimmt. Bei der Einstufung einer konkreten Person werden Ausbildung, Berufs- und Lebenserfahrung sowie die Lage auf dem Arbeitsmarkt berücksichtigt. In der Regel steigen Beschäftigte mit guten Leistungen innerhalb der Lohnklasse beständig die Erfahrungsstufen hinauf und haben so einen realen Lohnzuwachs. Genauere Vorschriften zur Berücksichtigung z.B. von durch Familienpflichten erworbenen Kompetenzen gibt es nicht. Bei der Lohnentwicklung werden auch die Ergebnisse der Mitarbeitendenbeurteilung herangezogen; je besser die Beurteilung, desto schneller kann der Lohn steigen. Über die Lohnentwicklung wird mit dem sog. Begleitausschuss der Sozialpartner verhandelt, in dem die Gewerkschaften und Personalverbände vertreten sind. Leistungsprämien können hier bis zu 15\% betragen.

Allgemein stellen zahlreiche empirische Studien die tatsächliche Motivationswirkung von Leistungsprämien stark in Frage (Kuhlmann/Wollmann 2013: 232 m.w.N.), zumal wenn sie nach dem Gießkannenprinzip verteilt werden (ebd.: 234 sowie Schweizer Interviews). In Deutschland sahen die interviewten Gleichstellungsbeauftragten wegen des komplexen Zusammenwirkens von dienstlichen Beurteilungen, geschlechtsspezifischer Nutzung von Teilzeit und Erwerbsunterbrechungen in den Leistungskomponenten auch ein gewisses Diskriminierungspotenzial.

Geschlechtsspezifische Einkommensunterschiede existieren auch in den Bundesverwaltungen, sie sind aber wesentlich geringer als in der Privatwirtschaft. In der Schweiz betrug der Gender Pay Gap beim monatlichen standardisierten Bruttolohn im Jahr 2010 12\%. Das ist weniger als in den Kantonen mit $16 \%$, aber mehr als in den Gemeinden mit $7 \%$, und wesentlich weniger 
als in der Privatwirtschaft mit 18\%. ${ }^{15}$ In Österreich waren es im Jahr 2011 im Bundesdienst 15\%. Diese Lücke divergiert nach Berufsgruppen: So liegt sie bei LehrerInnen bei 15\% und beim militärischen Dienst bei 31\% (BKA 2012). Seit Einführung der Einkommensberichtspflicht 2011 werden Einkommensunterschiede auch im öffentlichen Dienst vermehrt diskutiert. In Deutschland gibt es bisher nur einen Bericht zum geschlechtsspezifischen Verdienstunterschied im öffentlichen Dienst aus dem Jahr 2009. Aufgrund der zur Verfügung stehenden Datengrundlage sind diese Unterschiede systematisch geringer als der Gender Pay Gap, der für europäische Vergleiche verwendet wird. ${ }^{16}$ Die ausgewiesenen Abstände sind sehr gering, nämlich etwa $1 \%$ im Bundesdienst, $8 \%$ in den Ländern und $11 \%$ in den Kommunen BMFSFJ (2009: 12; zur Methodik vgl. S. 7-10). Sie sind für Tarifbeschäftigte höher als für BeamtInnen und in Bereichen mit hohem Frauenanteil ausgeprägter (was mit längeren Erwerbsunterbrechungen z.B. im Schuldienst erklärt wird). Dennoch scheint 1\% im Bundesdienst angesichts der Geschlechterverteilung auf den verschiedenen Hierarchieebenen nicht so recht plausibel.

Abb. 3.3: Lohnunterschiede zwischen den Geschlechtern beim Bund und in der Gesamtwirtschaft (2010, in \%)

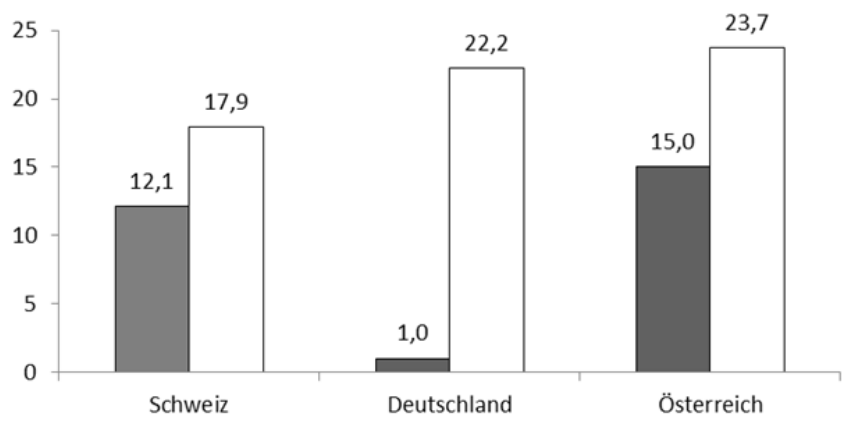

Bundesverwaltung $\square$ Gesamtwirtschaft

Quelle: Bundesverwaltung: Schweiz: Strub/Stocker (2010); Deutschland: BMFSFJ (2009); Österreich: BKA (2012); Zahlen für Gesamtwirtschaft 2011 unter http://epp. eurostat.ec.europa.eu/tgm/table.do?tab=table\&init $=1 \&$ plugin $=1 \&$ language $=$ en $\&$ pcode $=$ tsdsc340 (Zugriff: 28.02.2014)

$15 \mathrm{Vgl}$. http://www.bfs.admin.ch/bfs/portal/de/index/themen/20/05/blank/key/loehne/privatwirt schaft.html sowie http://www.bfs.admin.ch/bfs/portal/de/index/themen/20/05/blank/key/ loehne/oeffentlicher_sektor_bund_html (Zugriff: 03.09.2013). Werden die Lohnunterschiede nach europäischer Art berechnet, also bezogen auf den Bruttostundenlohn, ist er leicht höher. Er betrug 2010 in der Schweiz 19,1\%; vgl. http:/www.bfs.admin.ch/bfs/ portal/de/index/themen/20/05/blank/key/int_vergleiche.Document.69033.xls (Zugriff: 03.09.2013).

16 Der Gender Pay Gap wird auf Grundlage der durchschnittlichen Brutto-Stundenverdienste berechnet. Der Verdienstabstand für den Bundesdienst wurde als durchschnittlicher Verdienst aller bezahlter Stunden berechnet. 
Allgemein gesprochen kann ein Teil der Einkommensdifferenzen auf Unterschiede in Qualifikation, Dienstalter bzw. Berufserfahrung und Dienstverhältnis (verbeamtet vs. angestellt), auf Überstunden und berufliche Position zurückgeführt werden. Somit ist ein Teil der Lohnlücke objektiv erklärbar und nicht an sich geschlechterdiskriminierend. Die Lücke geht allerdings auf gesellschaftliche Strukturen zurück, die ihrerseits diskriminierend sind. In der Schweiz wurden die diskriminierenden Lohnunterschiede auf etwa $40 \%$ geschätzt, somit beträgt die Lohndiskriminierung beim Bund $4 \%$ und in der Privatwirtschaft 9\% (Strub/Stocker 2010: II). Der österreichische Gleichbehandlungsbericht hält fest, dass das Alter, die Anzahl der Überstunden, der Eintritt in den Bundesdienst oder die Zuordnung in den Gehalts- bzw. Entlohnungsstufen den größten Einfluss auf Einkommensunterschiede zwischen Männern und Frauen im Bundesdienst haben (BKA 2012: 22). Insgesamt zeichnet sich weibliche Beschäftigung im öffentlichen Dienst dadurch aus, dass sie öfter eine qualifizierte und damit besser bezahlte Erwerbstätigkeit darstellt als in der Privatwirtschaft (vgl. Strub/Stocker 2010; BMFSFJ 2009).

\subsection{Frauen und Männer in den Hierarchien des Bundesdienstes}

Um gleichstellungspolitische Entwicklungen beurteilen zu können, ist ein Blick auf die Verteilung von Frauen und Männern auf den verschiedenen Hierarchiestufen wichtig. Wir greifen hier auf die aktuellsten OECD-Zahlen von 2013 zurück, die mit klaren Kriterien von den Personalabteilungen in den jeweiligen Verwaltungen erhoben wurden. Die folgenden Zahlen beziehen sich jeweils auf den Bundesdienst (=, ,entral government"). ${ }^{17}$

In allen drei Ländern gibt es eine typische, wenn auch nicht überraschende vertikale Gechlechtersegregation: Je höher der Lohn und je höher die Position, desto weniger Frauen sind anzutreffen. Dabei ist eine langsame, aber stetige Aufholbewegung der Frauen auszumachen.

Sowohl die OECD-Daten wie die jeweiligen Daten aus den LänderFallstudien (welche nicht direkt miteinander vergleichbar sind) zeigen eine klare - und bekannte - Tendenz: Die Männeranteile unter den Beschäftigten sind umso höher, je höher die Hierarchiestufe ist. Dabei zeigt Österreich im Vergleich die höchsten, die Schweiz die niedrigsten Frauenanteile an den Führungspositionen. Auch hier ist eine langsame, wenn auch stetige Auf-

17 Hierarchiestufen und Führungspositionen sind in den drei Ländern nicht direkt vergleichbar und hätten sich von uns kaum miteinander vergleichbar machen lassen. In den jeweiligen Länderkapiteln gehen wir allerdings noch näher auf Führungspositionen und Dienstlaufbahnen ein. Die dortigen Zahlen sind wegen der unterschiedlichen Erhebungsmethoden nicht direkt mit der OECD-Studie vergleichbar. 
wärtsbewegung in allen drei Ländern festzustellen. So stieg zwischen 2002 und 2011 der Schweizer Frauenanteil im Topmanagement von 7\% auf 12\% und im mittleren Management von einem Fünftel auf gut ein Viertel. Die Steigerungsraten waren in jenen Departementen relativ gesehen am größten, die überdurchschnittliche Frauenanteile aufwiesen; im Verteidigungsdepartement VBS hingegen gelang keine substanzielle Steigerung. In Österreich wiesen die Bundesgleichstellungsberichte eine ungefähre Verdoppelung des Frauenanteils in den „höherwertigen Verwendungen“ (nach Verwendungsgruppen im Besoldungsschema) von 15\% auf 32\% zwischen 2003 und 2011 aus. Die Anstiege schwankten ebenfalls stark zwischen den Ministerien. In Deutschlands obersten Bundesbehörden (dem ,zentralen Teil“" des Bundesdienstes) wuchs der Frauenanteil an allen ,leitenden Funktionen“ (Referats-, Unterabteilungs- und Abteilungsleitungen) gemäß der Frauenförder- bzw. Gleichstellungsstatistik zwischen 2002 und 2011 von $15 \%$ auf $26 \%$; davor hatte der Beginn der rot-grünen Koalition 1998 eine besondere Schubwirkung entfaltet.

Abb. 3.4: Frauenanteil in verschiedenen Beschäftigungsgruppen im Bundesdienst (2010)

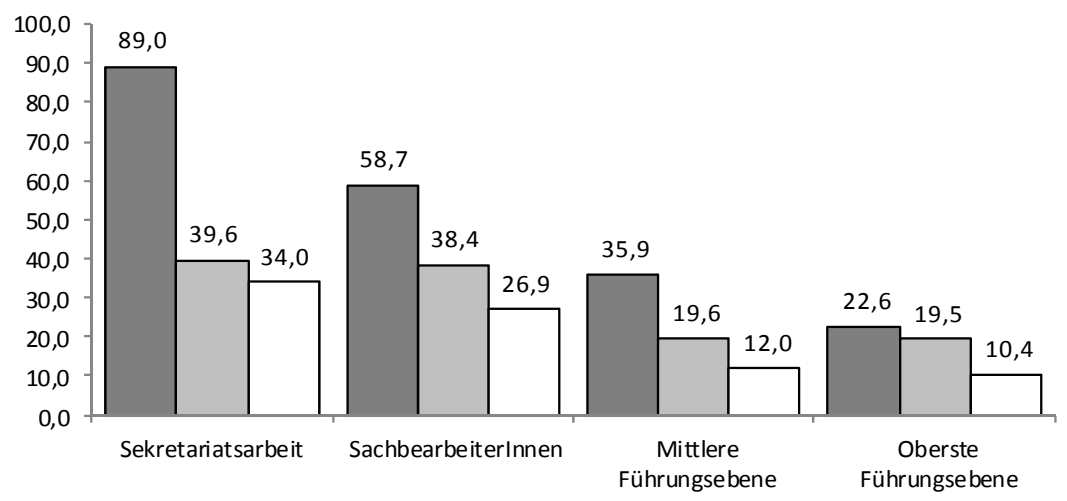

$\square$ Österreich $\square$ Deutschland $\square$ Schweiz

- Oberste Führungsebene: Positionen unterhalb der Minister- bzw. Ministerstellvertreterebene

- Mittlere Führungsebene: Positionen zwischen oberster Führungsebene und Führungsverantwortung für mindestens drei Beschäftigte, z.B. LeiterInnen von Gruppen oder Abteilungen

- SachbearbeiterInnen: Positionen zwischen Managementfunktionen und administrativer Unterstützung

- Sekretariatstätigkeiten: Beschäftigte in Verantwortung administrativer Tätigkeiten und allgemeiner Bürounterstützung

Quelle: OECD (2013a: 122f.) 


\subsection{Zwischenfazit: Gleichstellungspolitik im Spannungsfeld zwischen gleichstellungspolitischen Anforderungen und traditionellen Strukturen}

Im Vergleich zur Privatwirtschaft bietet der öffentliche Dienst gute Voraussetzungen für eine aktive betriebliche Gleichstellungspolitik. Staatliche Tätigkeiten unterliegen der Rechtsbindung, nichts darf willkürlich sein, und darum muss sich auch die Personalpolitik an Verfassung und Gesetz halten. Der öffentliche Dienst, und dabei auch der untersuchte Bundesdienst, bietet daher nach wie vor eine höhere Arbeitsplatzqualität sowie qualifiziertere Beschäftigungsmöglichkeiten für Frauen und somit geringere geschlechtsspezifische Lohnunterschiede. Allerdings deutet sich auch an, dass es nach wie vor substanzielle Inkompatibilitäten zwischen den zwei Logiken des Personalrechts auf der einen und des Gleichstellungsgesetzes auf der anderen Seite gibt, sodass die quasi automatische Gleichstellung von Frauen auch hier nicht erwartet werden kann. Ziehen wir zudem in Betracht, dass sich historisch die Beschäftigungspolitik von einer grundlegenden Benachteiligung zu einer Förderung der Frauenerwerbstätigkeit gewandelt hat, können wir mit Gudrun Kling (1999: 600) davon sprechen, dass „der Staatsdienst zugleich Abbild und Vorbild gesellschaftlicher Grundmaximen seiner Zeit ist. 



\section{Betriebliche Gleichstellung in der schweizerischen Bundesverwaltung}

Gesine Fuchs ${ }^{1}$

\subsection{Von der Rückständigkeit zu ungleichzeitigen Fortschritten: Gleichstellungspolitische Kontextbedingungen}

\subsubsection{Veränderungen im schweizerischen Geschlechterregime}

In der Schweiz hat es seit 1970 substanzielle Veränderungen im Geschlechterregime gegeben (Fuchs 2012), die auf die Gewährung politischer Rechte für Frauen, die umfassende rechtliche Gleichstellung sowie Veränderungen von Erwerbs-, Familien- und Bildungsmuster ${ }^{2}$ zurückgeführt werden können. Dabei entwickelte sich die Gleichstellungspolitik in Auseinandersetzung mit einem Wohlfahrtsregime, das in der Forschung im Anschluss an EspingAndersens Typologie überwiegend als konservativ definiert wird (PfauEffinger 2005; Bambra 2007: 328 m.w.N.). Ein näherer Blick zeigt jedoch, dass der schweizerische Sozialstaat durch eine eigentümliche Mischung aus liberalen und konservativen Elementen gekennzeichnet ist (Häusermann/Zollinger 2014: 911-919) und die Politiken sich in den letzten Jahrzehnten stark verändert haben. Liberal ist die Schweizer Sozialpolitik, weil sie relativ gesehen nur wenige staatliche Leistungen vorsieht, die zudem häufig bedarfsgeprüft sind. So gab es bis 2004 keine gesetzlichen Lohnersatzleistungen bei Mutterschaft, obwohl ein achtwöchiges Arbeitsverbot nach der Niederkunft bestand. Konservativ ist der Schwerpunkt auf Transferleistungen wie Familienzulagen bei gleichzeitig geringen Investitionen in die Infrastruktur, etwa zur Kinderbetreuung. Vor 20 Jahren sorgten unregelmäßige schulische Stundenpläne auch noch für die institutionelle Verhinderung mütterlicher Erwerbstätigkeit. Hier ist ein starker Familialismus zu konstatieren: Kinder und

1 Ein herzliches Dankeschön geht an alle Interviewpartner und -partnerinnen, die sich die Zeit genommen haben, Einblick in ihre Arbeit und ihr Gleichstellungsverständnis zu geben. Das Eidgenössische Personalamt hat zudem mehrmals unbürokratisch Primärdaten zur Entwicklung des Personalbestands zur Verfügung gestellt, was differenzierte Auswertungen ermöglicht hat. Einige Interviews führte Isabelle Schläpfer, die auch einen Großteil der Gespräche verschriftlicht hat und die Ergebnisse mit mir diskutiert hat. Ohne diese Personen wäre die vorliegende Arbeit nicht möglich gewesen.

2 Frauen haben in den letzten Jahrzehnten bei der (Schul-)Bildung erheblich aufgeholt, bleiben aber häufiger als Männer ohne Berufsausbildung und wählen kürzere Ausbildungen. An der geschlechtsspezifischen Berufswahl hat sich in den letzten Jahren kaum etwas verändert, sodass die Segregation bestehen bleibt (vgl. Huber/Bergman 2013). 
die sozialen wie ökonomischen Folgen sind Privatsache (Wahl/Hardmeier 2009). Als konservativ ist auch die gemeinsame steuerliche Veranlagung von Eheleuten anzusehen und eine soziale Sicherung, die nach Beschäftigungsstatus abgestuft ist: Die sog. zweite private kapitalgedeckte Säule begünstigt Vollzeitarbeitende. Insgesamt stellen diese Faktoren auf das Modell des Alleinernährers ab.

Dabei haben sich die Beschäftigungsmuster deutlich verändert. Seit 1970 sind die Erwerbsquoten der Frauen von 40\% (vgl. Bühler/Heye 2005: 24) auf über drei Viertel angestiegen: 2014 waren 78,7\% aller Frauen zwischen 15 und 64 Jahren und 88,1\% aller Männer erwerbstätig. ${ }^{3}$ Dabei ist allerdings die weibliche Teilzeitquote mit 58,5\% (2013) sehr hoch, selbst im Vergleich zur traditionell hohen weiblichen Teilzeitbeschäftigung in Deutschland und Österreich (46,5\% bzw. 45,6\% im Jahr 2013). ${ }^{4}$ In der Schweiz hat sich somit ein modernisiertes Ernährermodell entwickelt: Nur noch knapp 30\% der Familien mit Vorschulkindern lebten 2013 nach dem traditionellen Modell, in dem der Vater in Vollzeit erwerbstätig ist und die Mutter sich ausschließlich um Haushalt und Kinder kümmert. 1970 waren es noch etwa 70\%. Heute arbeitet in der Hälfte der Haushalte mit kleinen Kindern der Vater Vollzeit, die Mutter Teilzeit. ${ }^{5}$ Dabei tragen in über drei Viertel der Paarhaushalte mit kleinen Kindern die Frauen die Hauptverantwortung für Haus- und Betreuungsarbeit. ${ }^{6}$ Die folgenden Daten verdeutlichen, dass dieses modernisierte Modell ein gemeinsamer Trend in den deutschsprachigen Ländern ist:

Studien zeigen, dass auch die junge Generation mütterliche Erwerbstätigkeit als ökonomisch und persönlich wichtig einstuft, diese aber den Anforderungen der Kinderbetreuung nachgeordnet sehen will (Schwiter 2011).

Seit 2005 haben weibliche Beschäftigte endlich das Recht auf 14 Wochen bezahlten Mutterschaftsurlaub, der bereits seit 1945 in der Verfassung verankert ist. Die relativ bescheidene Lösung (80\% Lohnersatz mit einem Höchstbetrag) gelang, als sich auch bürgerliche Parteien und Arbeitgeberverbände dafür einsetzten. Mit der Mutterschaftsversicherung, die wie die Renten- oder Arbeitslosenversicherung über Lohnabzüge finanziert wird, wurde deutlich, dass Nachwuchs nicht mehr ausschließlich als Privatangelegenheit gesehen wird, sondern es eine gesellschaftliche Verantwortung für Kinder und junge Mütter gibt. Seit Einführung der Mutterschaftsversicherung 2005 besteht überhaupt erst eine Grundlage, Elternurlaube zu fordern, was im nationalen Parlament auch zunehmend geschieht (genauer vgl. Fuchs 2015a). Außer in

3 Erwerbstätigkeit und Arbeitszeit - Indikatoren; vgl. http://www.bfs.admin.ch/bfs/portal/de/ index/themen/03/02/blank/key/erwerbsquote.html (Zugriff: 09.02.2015).

4 „Detaillierte Ergebnisse der SAKE“; vgl. http://www.bfs.admin.ch/bfs/portal/de/index/ themen/03/02/blank/data/03.html (Zugriff: 09.02.2015).

5 „Erwerbsmodelle in Paarhaushalten“; vgl. http://www.bfs.admin.ch/bfs/portal/de/index/ themen/20/05/blank/key/Vereinbarkeit/03.html (Zugriff: 09.02.2015).

6 „Erwerbsmodelle in Paarhaushalten“; vgl. http://www.bfs.admin.ch/bfs/portal/de/index/ themen/20/05/blank/key/Vereinbarkeit/02.html (Zugriff: 09.02.2015). 
einigen Großunternehmen und bei öffentlichen Arbeitgebern gibt es jedoch keine Ansprüche auf bezahlte oder unbezahlte Elternzeit, wie etwa in Deutschland oder Österreich (vgl. Fuchs 2008a; für die Bundesverwaltung s.u.). Der Kündigungsschutz für Mütter endet 16 Wochen nach der Geburt. Dennoch steigen Mütter heute seltener als früher ganz aus dem Erwerbsleben aus, sondern arbeiten kontinuierlich, aber häufig sehr kurz: 38\% der erwerbstätigen Mütter arbeiteten 2013 unter 50\%, 7\% sogar unter 20\%. ${ }^{7}$

Tab. 4.1: Beschäftigungsmuster in Prozent bei Familien mit Kindern unter 14 Jahren (2011)

\begin{tabular}{lcccc}
\hline & $\begin{array}{c}\text { Beide Eltern } \\
\text { Vollzeit }\end{array}$ & $\begin{array}{c}\text { Vollzeit/nicht } \\
\text { erwerbstätig }\end{array}$ & Vollzeit/Teilzeit & Anderes $^{*}$ \\
\hline Deutschland & 16,5 & 29,3 & 43,8 & 10,4 \\
Österreich & 20,8 & 27,5 & 44,3 & 7,4 \\
Schweiz & 8,0 & 24,4 & 40,2 & 27,4 \\
EU-25 & 44,6 & 30,5 & 18,0 & 6,9 \\
OECD & 36,6 & 33,3 & 23,6 & 6,9 \\
\hline
\end{tabular}

* Hierunter fallen z.B. zwei Elternteile in Teilzeit und zwischen 1\% und 5\% Eltern ohne Erwerbstätigkeit.

Quelle: OECD Family Database (unter http://www.oecd.org/social/family/database.htm\# INDICATORS, Grafik LMF2.2A; Zugriff: 09.02.2015)

Die Zahl der Kinderkrippen und Horte ist in den letzten 25 Jahren stark gewachsen, wozu auch ein Bundesprogramm zur Anschubfinanzierung von Angeboten mit einem Volumen von insgesamt 360 Mio. Franken beigetragen hat. ${ }^{8}$ Dennoch kommt eine aktuelle Studie zum Schluss, dass nur für $11 \%$ der Vorschulkinder und für $8 \%$ der Schulkinder ein Vollzeitbetreuungsplatz zur Verfügung steht. Dabei sind die Plätze vor allem in städtischen Regionen und besonders in der West- und Nordschweiz konzentriert. Insgesamt werden etwa 30\% der bis zu Zwölfjährigen mindestens eine Stunde pro Woche in einem institutionalisierten Angebot betreut. ${ }^{9}$ Die Studie wies einen Zusammenhang zwischen der Erhöhung des Betreuungsangebots und höheren Vollzeit-Erwerbsquoten von Müttern nach und kam daher zum Schluss, dass Kinderbetreuungsangebote die Gleichstellung der Geschlechter fördern (Stern et al. 2013). Die Finanzierungsmodalitäten und die Chancen, ein Kinderbetreuungsangebot in einer Gemeinde durchzusetzen, schwanken je nach politischen Mehrheitsverhältnissen und den Vorschriften der jeweiligen Kan-

7 „Detaillierte Ergebnisse der SAKE“; vgl. http://www.bfs.admin.ch/bfs/portal/de/index/ themen/03/02/blank/data/03.html, Tabelle T 03.02.01.15 (Zugriff: 09.02.2015).

$8 \quad$ Vgl. http://www.bsv.admin.ch/praxis/kinderbetreuung/01153/index.html (Zugriff: 09.02.2015).

9 Vgl. http://www.bfs.admin.ch/bfs/portal/de/index/themen/01/04/blank/key/04/01.html (Zugriff: 09.02.2015). 
tone. Hinzu kommen schichtspezifische Nutzungsmuster von Kinderbetreuungsangeboten (Widmer/Zollinger 2014; Widmer et al. 2014a). Zugleich bestehen durch die Ausgestaltung von einkommensabhängigen Betreuungstarifen, der Subventionspraxis und dem Steuerrecht (gemeinsame Veranlagung, Deckelung der Abzüge für effektive Betreuungskosten) häufig Fehlanreize für die substanzielle Erwerbstätigkeit von Müttern in Paarhaushalten. Modellrechnungen für die Kantone Basel-Stadt und Zürich (Schwegler et al. 2011: 34-40, 49-60; siehe auch schon Bütler 2007) zeigen, dass bei zwei Kindern in Betreuung je nach Parametern eine Vollzeiterwerbstätigkeit des zweiten Elternteils das verfügbare Einkommen nur um 1.000 Franken erhöht; bei Teilzeit kann das zusätzliche Einkommen sogar ganz aufgezehrt werden. Der Ausbau der Kinderbetreuung wird in Parlament und öffentlicher Diskussion zunehmend mehrheitsfähig. Er wird dabei immer öfter mit ökonomischen Argumenten gestützt: Der Arbeitsmarkt könne nicht auf gut ausgebildete Frauen verzichten, und volkwirtschaftlich würden die Ausbildungskosten verschwendet, wenn Frauen lange zu Hause blieben oder nur wenig arbeiten könnten. Dabei ist in der öffentlichen Diskussion eine Elitenfixierung unverkennbar, also ein fast ausschließlicher Bezug auf hoch qualifizierte Frauen. Ein kursorischer Blick auf die Anstellungsbedingungen in der Kinderbetreuung offenbart schließlich ein Paradox: Gleichstellungsfortschritte, die durch den Ausbau der Kinderbetreuung ermöglicht werden, nämlich eine höhere und qualifiziertere Erwerbstätigkeit von Müttern, werden konterkariert durch prekäre Beschäftigungsbedingungen in den Betreuungseinrichtungen. Löhne und Anstellungsumfang des vornehmlich weiblichen Personals ermöglichen ihnen keine eigenständige Existenzsicherung (Widmer et al. 2014b).

\subsubsection{Rechtliche Grundlagen beruflicher Gleichstellungspolitik}

Nachdem die Schweizer Frauen 1971 das Wahlrecht bekamen und 1981 eine Volksinitiative für einen Gleichstellungsartikel in der Verfassung angenommen wurde, wurden in den 1980er und 1990er Jahren im Wesentlichen alle direkten rechtlichen Diskriminierungen beseitigt (vgl. Fuchs 2009: 584-587; genauer dazu Fuchs 2015b). Die Bundesverfassung postuliert einen Auftrag des Staates für die tatsächliche Gleichstellung:

„Mann und Frau sind gleichberechtigt. Das Gesetz sorgt für ihre rechtliche und tatsächliche Gleichstellung, vor allem in Familie, Ausbildung und Arbeit. Mann und Frau haben Anspruch auf gleichen Lohn für gleichwertige Arbeit.“ (Art. 8 Abs. 3 BV; Hervorhebung GF)

Auf dieser Grundlage haben Bund, 18 von 26 Kantonen und einige Städte staatliche Gleichstellungsstellen eingerichtet. Sie wurden vor allem zwischen Ende der 1980er bis Mitte der 1990er Jahre gegründet. Die Mehrheit der 
Stellen hat einen doppelten Auftrag, d.h. ist sowohl für die Förderung der tatsächlichen Gleichstellung innerhalb des Betriebs ,öffentliche Verwaltung“ als auch in der gesamten Bevölkerung zuständig. In der Regel gehören zu ihren Aufgaben: Beratung und Information für Behörden und Private, Öffentlichkeitsarbeit, Stellungnahmen in Gesetzgebungsverfahren, Schaffung von Grundlagen- und Umsetzungswissen durch Untersuchungen, Instrumente und Projekte sowie Koordination und Vernetzung. Gleichstellung im Recht, bei der Erwerbs- und Care-Arbeit sowie in Schule und Ausbildung, Förderung der Vereinbarkeit von Familie und Beruf und die Bekämpfung häuslicher Gewalt sowie der Bereich politische Partizipation und Repräsentation bilden dabei thematische Arbeitsschwerpunkte. Das Eidgenössische Büro für die Gleichstellung (EBG) besteht beispielsweise seit 1988 und ist mittlerweile ein eigenes Amt. Es bekam 1996 mit einem Artikel im Gleichstellungsgesetz eine rechtliche Grundlage: Es ,fördert die Gleichstellung der Geschlechter in allen Lebensbereichen" (Art. $16 \mathrm{GlG}$ ) und ist zuständig für Informationsarbeit, Beratung von Behörden und Privaten, ist an Projekten nationaler Bedeutung beteiligt und vergibt die Gelder aus dem Förderprogramm der Finanzhilfen (Art. 15 GlG). Im Gegensatz zu den meisten anderen Stellen ist das Eidgenössische Büro nicht für die betriebliche Gleichstellung in der Verwaltung zuständig. Um 1995 und um 2003 wurden Fachstellen durch politische Initiativen vermehrt in Frage gestellt (Scheidegger 2008; Seitz 2010: 2-5); auch heute stehen sie unter Legitimationsdruck. ${ }^{10}$ Insgesamt haben die Stellen nur wenige Kontroll- oder Anhörungsrechte, und die Personalausstattung ist für diese transformatorische Querschnittsaufgabe sehr gering. Konkrete bundesrechtliche Vorgaben gibt es nicht. Diese Gleichstellungsarbeit ist wenig rechtlich verankert und institutionalisiert, was im Vergleich zu Deutschland besonders auffällt, wo die Rechtslage sukzessive ausdifferenziert wurde (Lang 2009; Rudolph/Schirmer 2004: 73-75). Entsprechend sind Strategien wie Gender Mainstreaming oder Gender Budgeting nicht über unverbindliche Pilotprojekte hinaus eingesetzt worden (vgl. EBG 2014).

Für die Gleichstellung im Erwerbsleben ist das Gleichstellungsgesetz (GlG) von 1996 der wesentliche Pfeiler. Das GlG $^{11}$ ist ein eigenes zivilrechtliches Gesetz, das einheitlich für alle unselbstständigen Erwerbsarbeitsverhältnisse gilt, seien sie privat- oder öffentlich-rechtlich. Es verbietet sexuelle Belästigung und direkte wie indirekte Diskriminierung aufgrund des Ge-

10 Es ist umstritten, inwiefern rechtliche Grundlagen wie Gleichstellungsartikel in der Bundesund in vielen Kantonsverfassungen sowie internationalen Abkommen wie der CEDAW den Staat zu Gleichstellungsinstitutionen verpflichten können. In einem neueren Urteil zu einer Beschwerde gegen Regierung und Parlament des Kantons Zug wegen der Nichtfortführung der Kommission für Chancengleichheit von Frau und Mann hat das Bundesgericht festgehalten, dass es zwar eine Pflicht zu staatlichen Gleichstellungsmaßnahmen gibt, die konkreten Aufgaben und Instrumente jedoch nicht vorgegeben seien (1C.549/2010 vom 21. November 2011; vgl. Medici 2012).

11 Für mehr Informationen vgl. Fuchs (2008b) und http://www.gleichstellungsgesetz.ch. 
schlechts, postuliert die Beweislasterleichterung und ermöglicht die Verbandsklage. Das Gesetz führte zudem niedrigschwellige und kostenlose Schlichtungsverfahren ein, die eine vorgerichtliche Lösung des Konflikts ermöglichen sollen, die 2011 national vereinheitlicht wurden. Eine weitere Besonderheit ist die sog. Untersuchungsmaxime: Das Gericht muss bei einer Klage nach Gleichstellungsgesetz nach Bedarf von sich aus Beweise erheben, z.B. Zeugen befragen oder Gutachten anordnen; normalerweise müssen in einem Zivilprozess nämlich die Klagenden alle wesentlichen Fakten für ihre Forderung beibringen. Das Gleichstellungsgesetz hat gerade bei Lohnklagen eine stärkere Wirkung als die entsprechenden Regelungen in Deutschland und in Österreich entfaltet (vgl. Fuchs et al. 2009). Die größte Schwäche ist aber der reaktive und individualistische Charakter. Das Verbot der Diskriminierung muss von den Diskriminierten ex post mobilisiert werden. Da es kaum Kontrollen gibt, ist das Risiko gering, für Diskriminierung zur Verantwortung gezogen zu werden. Paradoxerweise scheint via Verbandsklage der kollektive Rechtsschutz vor Diskriminierung für gewerkschaftlich gut organisierte Gruppen von qualifizierten weiblichen Beschäftigten stärker zu sein als für Einzelpersonen (Fuchs 2013: 12). Schließlich ist die schweizerische Rechtslage hinter der Entwicklung in der Europäischen Union zurückgeblieben. ${ }^{12}$ Versuche, den Diskriminierungsschutz auf andere Merkmale wie Herkunft, Alter oder Behinderung und auf andere Bereiche als die Erwerbsarbeit auszuweiten, sind bisher erfolglos geblieben.

Teilweise im Rahmen des Gleichstellungsgesetzes (mit den sog. Finanzhilfen, Art. 15 GlG; vgl. http://www.topbox.ch) wurden seit Mitte der 1990er Jahre konkrete Maßnahmen zur Förderung der beruflichen Gleichstellung entwickelt, etwa Beratungsstrukturen für eine bessere Vereinbarkeit oder Diagnoseinstrumente zur Entdeckung von Lohndiskriminierung, von denen Logib das bekannteste ist und auch im Ausland rezipiert und adaptiert wurde (zur Diskussion vgl. Tondorf 2010). Mit Logib kann das Eidgenössische Büro für Gleichstellung ganz vereinzelt die Einhaltung der Lohngleichheit in Betrieben prüfen, die sich um öffentliche Aufträge des Bundes bewerben. ${ }^{13}$ Aufgrund eines Rechtsvergleichs zur Durchsetzung von Lohngleichheit und eines Vergleichs nationaler Durchsetzungsinstrumente in anderen Bereichen (vgl. Müller 2013) schlug der Bundesrat im Oktober 2014 eine Pflicht für innerbetriebliche Lohnanalysen in Unternehmen mit mehr als 50 Mitarbeitenden vor. ${ }^{14}$ Zurzeit stehen jedoch das gesetzliche Diskriminierungsverbot

12 Zwar wird immer noch eine Angleichung an den Rechtsstand der EU angestrebt (,autonomer Nachvollzug"), wo dies ökonomisch geboten und sinnvoll erscheint. Dies gilt offenbar nicht für das Antidiskriminierungsrecht.

132014 wurden neue Stellen für Lohngleichheit geschaffen.

14 Vgl. http://www.news.admin.ch/message/index.html?lang=de\&msg-id=54905 (Zugriff: 09.02.2015). Dabei soll die Kontrolle der betriebsinternen Analyse durch Dritte (Revisionsstelle, staatlich anerkannte Selbstregulierungsorganisation) erfolgen. Es soll veröffentlicht werden, ob Lohnunterschiede oberhalb der Toleranzschwelle gefunden wurden, nicht aber 
und die Sensibilisierungs-, Förder- und Informationsinstrumente unverbunden nebeneinander. Es gibt keine direkten Anreize oder Sanktionsdrohungen zum Einsatz dieser Instrumente (genauer dazu: Fuchs 2015b).

Das Gleichstellungsgesetz präsentiert sich als ein liberales Antidiskriminierungsgesetz, in dem es die Rechtsgleichheit der Individuen betont; es enthält kaum Schutzbestimmungen und wird nicht flankiert von Leistungsansprüchen, wie es in einem ,expanding opportunities"-Ansatz der Frauenförderung in Deutschland und Österreich typisch ist (vgl. von Wahl 1999), also etwa einem ausgebauten Kündigungsschutz, Rückkehr- und Beschäftigungsgarantien oder Elternurlaub.

Die Lebensmodelle und das Geschlechterregime in der Schweiz befinden sich im Umbruch. Politisch und sozial sind in den letzten 25 Jahren substanzielle Fortschritte in der Gleichstellung der Geschlechter zu verzeichnen, ohne dass sich jedoch ein konsistentes und kohärentes neues Policy-Regime herausbilden würde. Die Aussichten für eine konsistente, an einem emanzipatorischen Leitbild ausgerichtete Gleichstellungspolitik sind systematisch begrenzt: Hohe Autonomie von Gemeinden und Kantonen ergeben Koordinationsschwierigkeiten, begrenzen aber auch die Reichweite nationaler Politiken. Allparteienregierungen und eine starke Orientierung an Ausgleich, Kompromiss und Konsens erfordern übergreifende Koalitionen, sodass ,die reine Lehre" kaum in ein Gesetz zu gießen ist (vgl. Fuchs 2009: 581-584). Erschwerend kommt hinzu, dass in der politischen Debatte zwar alle die Gleichstellung grundsätzlich bejahen, aber kaum argumentativ für die Gleichstellung gefochten wird. Die weiter bestehenden Wertkonflikte werden nicht ausgetragen. Sie sind nur indirekt sichtbar, etwa im Streit um institutionelle Zuständigkeiten und die Finanzierung von Maßnahmen (Fuchs 2015a; Häusermann/Zollinger 2014: 930-931). Insofern ist es eine interessante Frage, ob ein Arbeitgeber, nämlich der Bund, eine kohärente und konsistente Politik umsetzen kann und ob er mit Leistungen für die Beschäftigten über eine liberale Antidiskriminierungspolitik hinausgeht. Plausibel scheint zumindest, dass auch diese Politik auf Eliten fokussiert, handelt es sich bei der Bundesverwaltung doch zu einem großen Teil um anspruchsvolle Arbeitsplätze.

die Höhe des Lohnunterschieds und nicht die allenfalls ergriffenen Gegenmaßnahmen. Unter Umständen käme auch eine subsidiäre Meldepflicht der Prüforganisationen in Frage, wenn Unternehmen Empfehlungen nicht umsetzen und weiter diskriminieren. Publizistisch wird seitdem verstärkt argumentiert, der geschlechterdiskriminierende Teil des Lohnunterschieds sei kleiner oder nicht vorhanden (vgl. Wirz 2014). 


\subsection{Frauen und Männer beim Bundespersonal: Langsame Feminisierung und starke Segregation}

Die Entwicklungen der letzten Jahre beim Bundespersonal zeigen einen langsamen, doch stetigen Anstieg beim Anteil der weiblichen Beschäftigten, wobei die höchsten Führungspositionen weiterhin stark von Männern dominiert sind. Gleichzeitig bietet die Bundesverwaltung im Vergleich zur Privatwirtschaft überdurchschnittlich viele Möglichkeiten für gut qualifizierte Frauen zu einer adäquaten Beschäftigung. Die Entwicklungen beim Frauenanteil insgesamt und in Führungspositionen variieren stark nach Departement (und einzelnem Amt). Die horizontale Segregation zwischen Berufsfeldern hält sich hartnäckiger als die vertikale Segregation, was mit gesamtwirtschaftlichen Tendenzen in der Schweiz seit 1970 übereinstimmt (Charles 2005).

Von den 324.000 Personen (in Vollzeitäquivalenten VZÄ), die 2008 in der schweizerischen öffentlichen Verwaltung arbeiteten, waren gut $10 \%$ in der Bundesverwaltung tätig. ${ }^{15}$ Obwohl die Kompetenzen des Bundes in der Schweiz relativ klein sind - insbesondere Verteidigung, auswärtige Beziehungen, Energie und Kommunikation - (Koller 2013: 504), gehört die Bundesverwaltung $\mathrm{zu}$ den größten Arbeitgebern ${ }^{16}$ im Land: 2011 waren dort 36.387 Personen beschäftigt, die sich 33.054 Arbeitsplätze teilten. ${ }^{17}$ Der Frauenanteil stieg zwischen 2002 und 2011 nur langsam um 3,2 Prozentpunkte von $28,4 \%$ auf $31,6 \%$. Damit sind in der Bundesverwaltung anteilig weniger Frauen als im gesamten öffentlichen Dienst in der Schweiz beschäftigt, wo mehr Frauen in den Bereichen Gesundheit, Soziales und Bildung arbeiten. ${ }^{18}$ Der Anstieg des Frauenanteils in der Bundesverwaltung ist in jenen Ämtern und Einheiten stärker, die bereits 2002 einen signifikanten Frauenanteil hatten oder sogar eine Frauenmehrheit. Umgekehrt ist es in den letzten Jahren nicht gelungen, den Frauenanteil an den Beschäftigten in stark männerdominierten Ämtern signifikant zu steigern. Sowohl die Abschwächung der vertikalen Segregation - mehr Frauen in Führungspositionen - als auch eine stärkere Durchmischung der Aufgabenbereiche scheinen hier als Aufgabe betrieblicher Gleichstellung auf.

15 Über $40 \%$ arbeiteten bei den Kantonen, ein gutes Drittel bei den Gemeinden und noch einmal gut 10\% in öffentlichen Körperschaften (Koller 2013: 502f.).

16 Zum Vergleich: 2012 gab es in der Schweiz nur 1.256 privatwirtschaftliche Unternehmen mit mehr als 250 Beschäftigten, aber 556.000 Firmen, die kleiner waren („Daten - Statistik der Unternehmensstruktur STATENT 2012“; http.//www.bfs.admin.ch/bfs/portal/de/index/ themen/06/02/blank/data.html (Zugriff: 02.09.2014).

17 Alle Angaben beruhen, wenn nicht anders angegeben, auf eigenen Berechnungen mit dem Datensatz des Eidgenössischen Personalamts (liegt der Verfasserin vor). Neuere Daten sind aggregiert und lassen keine genauen Angaben zur Geschlechterverteilung zu.

18 Der durchschnittliche Frauenanteil beträgt 52\%. 19\% aller erwerbstätigen Frauen, aber 13\% der Männer arbeiten in der öffentlichen Verwaltung (vgl. Koller 2013: 512-517). 


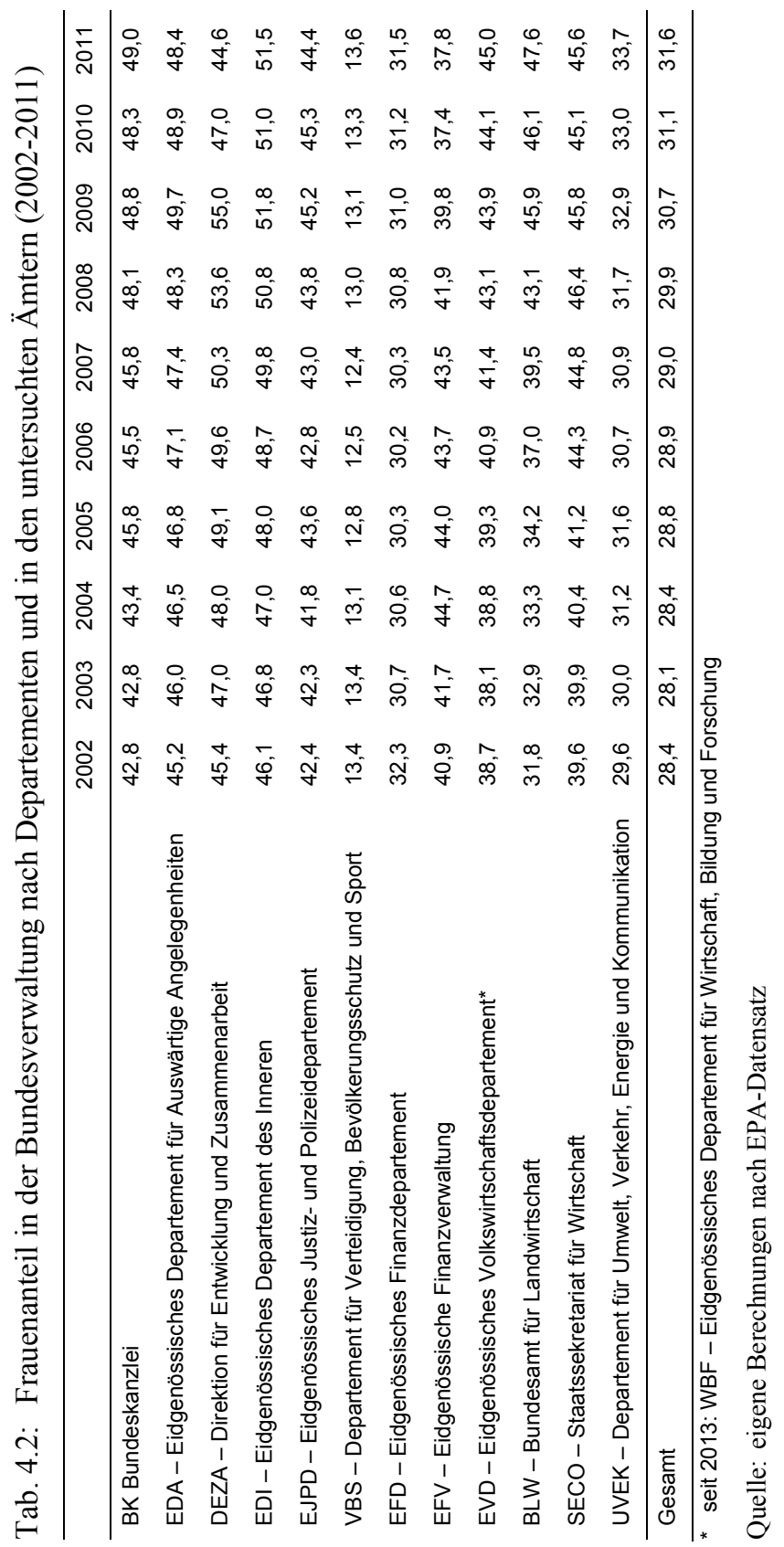


In der Bundesverwaltung gibt es vor allem qualifizierte und hoch qualifizierte Beschäftigung, und dieser Anteil steigt kontinuierlich: In den hier näher untersuchten Ämtern (Bundesamt für Landwirtschaft, Finanzverwaltung, Staatssekretariat für Wirtschaft und Direktion für Zusammenarbeit und Entwicklung) sind zwischen 51\% und 67\% der Beschäftigten in die Lohnklasse 24 oder höher eingestuft, also im ,mittleren Management“. Zwischen 75\% und $85 \%$ sind in die Lohnklasse 18 oder höher eingestuft. Hingegen hat sich in den letzten zehn Jahren die Beschäftigung in den untersten Lohnklassen 1 11 in der gesamten Bundesverwaltung halbiert. Diese Tendenz ist nicht ganz so ausgeprägt in der Gesamt-Bundesverwaltung, wo 2011 29\% im ,mittleren Management" tätig waren, wie Abb. 4.1 zeigt:

Abb. 4.1: Beschäftigung nach Lohnklassen, gesamte Bundesverwaltung (2002 und 2011)
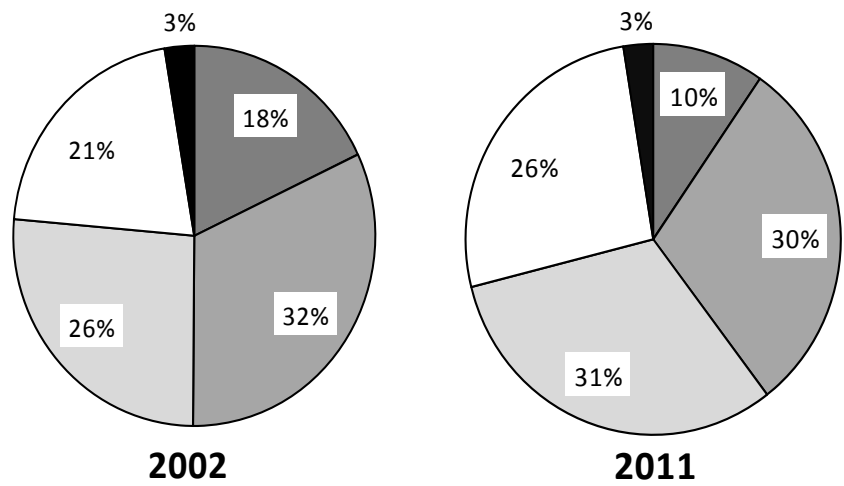

口LK 1-11

ㄴK $12-17$

口LK $18-23$

口LK 24-29

LK 30-38

Quelle: eigene Berechnungen nach EPA-Datensatz

Wegen dieser Qualifikations- und Aufgabenstruktur werden in der Bundesverwaltung im Schnitt um ein Fünftel höhere Durchschnittslöhne als in der Privatwirtschaft gezahlt, das gilt besonders für Frauen (Strub/Stocker 2010: II, 39). ${ }^{19}$

1994 leitete noch keine einzige Frau eine Amtsstelle, und nur fünf waren auf Stellvertretungsposten beschäftigt. 2011 standen hingegen neun Einheiten der direkten Bundesverwaltung Frauen vor, also einem knappen Sechstel aller Stellen, und neun waren auf Vizeposten tätig. ${ }^{20}$ Insgesamt ist eine klare vertikale Geschlechtersegregation in der Bundesverwaltung zu konstatieren: Je höher die Lohnklasse, desto niedriger ihr Frauenanteil.

19 Frauen verdienen in der Bundesverwaltung durchschnittlich $31 \%$ mehr als Frauen in der Privatwirtschaft. Bei den Männern sind es „,nur“ $26 \%$.

20 Berechnet nach Publicus (1994/95 und 2011). 
Abb. 4.2: Frauenanteil in Prozent nach Lohnklassen, gesamte Bundesverwaltung (2002-2011)

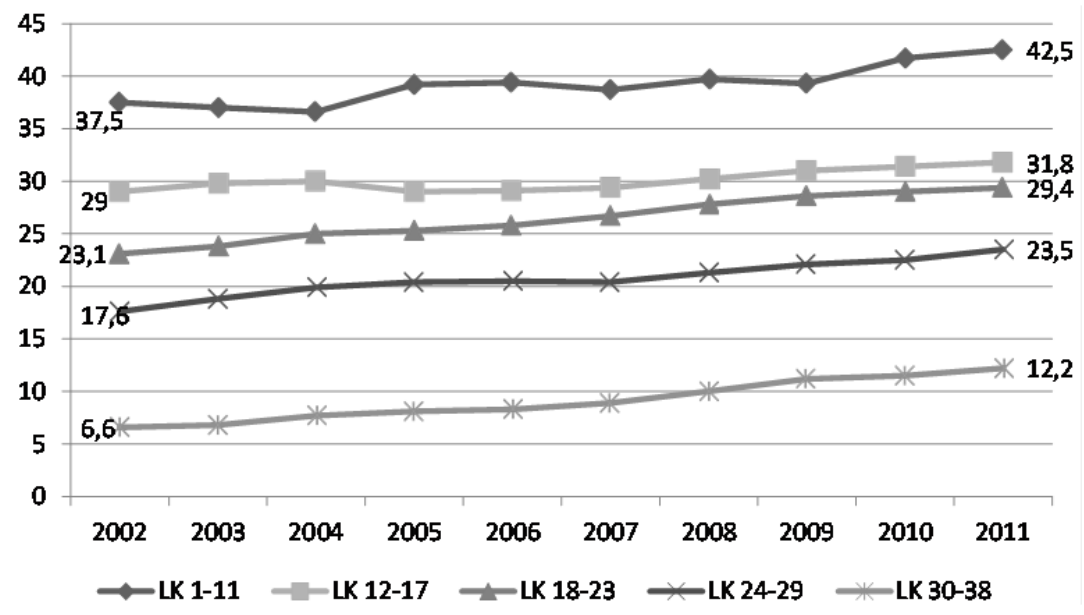

Quelle: eigene Berechnungen nach EPA-Datensatz

Zwischen den Departementen gibt es dabei erhebliche Unterschiede, wie der Vergleich der Frauenanteile für den mittleren und oberen Kader zwischen 2002 und 2011 zeigen.

Abb. 4.3: Frauenanteil in Prozent im mittleren Management (Lohnklassen 24-29) nach Departementen (2002 und 2011)

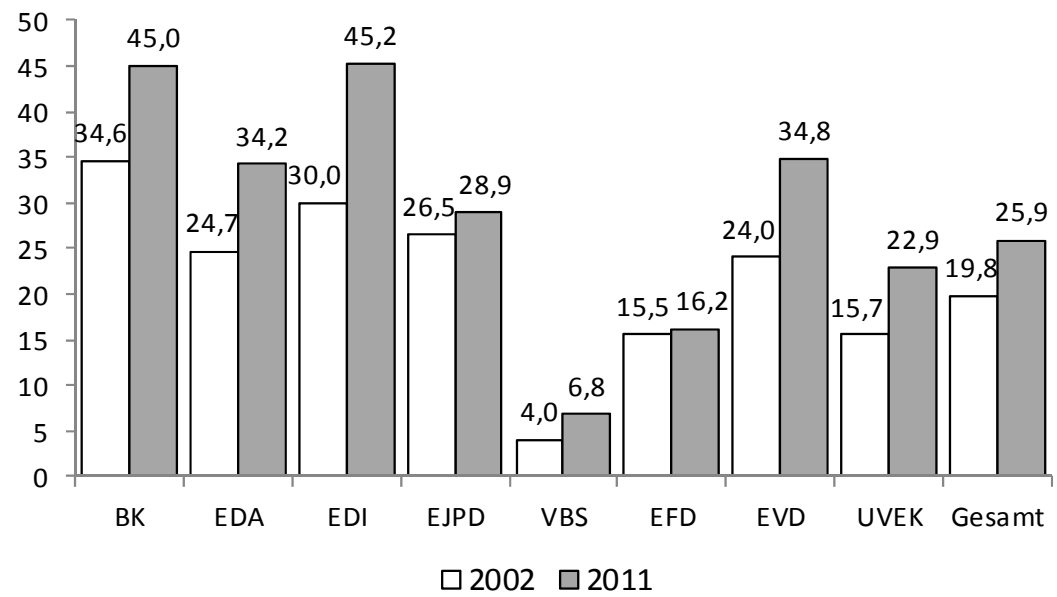

Quelle: eigene Berechnungen nach EPA-Datensatz; Abkürzungen vgl. Tab. 4.2 
Abb. 4.4: Frauenanteil in Prozent im oberen Management (Lohnklassen 30-38) nach Departementen (2002 und 2011)

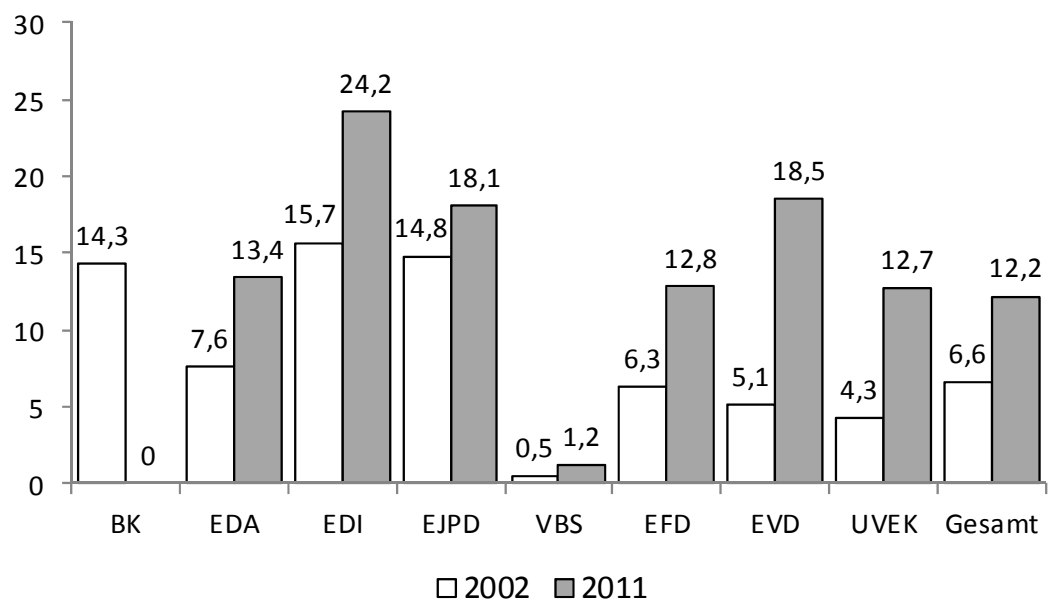

Quelle: eigene Berechnungen nach EPA-Datensatz

Der Abbau der vertikalen Geschlechtersegregation - mehr Frauen in qualifizierten und in Führungspositionen - wird je nach Aufgabenbereich durch die starke horizontale Segregation erschwert. Einige Departementen sind erfolgreicher bei der Rekrutierung von Frauen als andere. Inwiefern dies auf gleichstellungspolitische Strategien zurückzuführen ist, wird weiter unten untersucht.

\subsection{Institutionelle Politik in der schweizerischen Bundesverwaltung}

\subsubsection{Personalrechtliche Grundlagen betrieblicher Gleichstellung in der Bundesverwaltung}

Für die Gleichstellungspolitik in der Bundesverwaltung bilden die allgemeinrechtlichen Vorgaben und das Personalrecht die relevanten Eckpunkte. Das sind zum einen die Bundesverfassung und das Gleichstellungsgesetz, welches gleiches Recht für öffentliche und private Arbeitsverhältnisse setzt. ${ }^{21}$ Zum

21 Lediglich bei Verfahren und Kündigungsschutz gibt es wenige Unterschiede. Bis heute gibt es nur fünf Schlichtungsverfahren nach Gleichstellungsgesetz in der Bundesverwaltung (vgl. http://www.gleichstellungsgesetz.ch). 
anderen gibt es das Bundespersonalgesetz, das als Rahmengesetz im Sinne des New Public Management ausgestaltet wurde, mit seinen nachgeordneten Verordnungen und Weisungen. Das Bundespersonalrecht arbeitet mit allgemeinen Zielformulierungen und macht nur wenige prozessuale und materielle Vorschriften. Solche Ermessensspielräume sind im Konzept eines Rahmengesetzes angelegt und waren politisch gewollt (vgl. dazu das siebte Kapitel in diesem Buch). Für eine betriebliche Gleichstellungspolitik, die also über den gesetzlichen Minimalschutz des GlG hinausgeht, ist das Personalrecht entscheidend. Die große Bandbreite und Heterogenität aber von Maßnahmen in der Bundesverwaltung sind in diesen rechtlichen Grundlagen schon institutionell angelegt.

Vor dem Inkrafttreten des neuen Bundespersonalgesetzes (2002) liegt eine allgemeine frauen- und gleichstellungspolitische Pionier- und Aufschwungphase in der Schweiz. ${ }^{22}$ In der Bundesverwaltung zeigte sich dies durch die Abschaffung direkter Diskriminierung von Frauen im damaligen Beamtengesetz bei der Alterssicherung und den Sozialzulagen (1986). Auch konnten seitdem Teilzeitbeschäftigte beim Bund zu BeamtInnen ernannt und nicht nur angestellt werden (BBl 1986 II 313; vgl. Engeli 2011: 185). Anfang der 1990er Jahre wurde beim Eidgenössischen Personalamt im Finanzdepartement eine Stabsstelle für Frauenförderung eingerichtet mit dem Ziel, eine strategische Politik zu entwickeln. Diese Stelle war anfangs mit 120, dann mit 60 Stellenprozenten besetzt und besteht heute als Diversity-Stelle mit 110 Stellenprozenten fort. Die Stabsstelle erarbeitete Frauenförderpläne mit einer großen Bandbreite von Zielen und Maßnahmen, die sowohl auf strukturelle Veränderungen wie auch einzelnen Personen zielten. Einige Schwerpunkte dieser Programme sind heute noch relevant, wie die Förderung der Vereinbarkeit, die Kinderbetreuung, die Gleichstellung der Teilzeitarbeit, die Förderung von Jobsharing oder die Sicherung der Lohngleichheit, vor allem bei der Einführung des neuen Lohnsystems. In dieser Zeit wurden aber auch viele Grundlagen erarbeitet und Entwicklungen vorbereitet: Handreichungen zur Prävention sexueller Belästigung entstanden ebenso wie eine Handreichung zur wirksamen Erfassung von Schlüsselkompetenzen (Kadishi 2001) oder die

22 Dies zeigt sich in einem Institutionalisierungsschub, z.B. bei den Gleichstellungsstellen und bei vielen Frauenprojekten sowie dem Gang von Feministinnen in die Sozialdemokratische Partei. 1991 kam es zum Frauenstreik, der von wenigen Gewerkschafterinnen erdacht und innerhalb weniger Monate organisiert wurde, in dem sich aber gewerkschaftlich Organisierte und Mitglieder der Frauenbewegung trafen. 1993 führte schließlich die Nicht-Wahl der Sozialdemokratin Christiane Brunner in den Bundesrat zu einer politischen Mobilisierung für mehr Frauen in der Politik, in deren Folge einerseits eine andere Sozialdemokratin, Ruth Dreifuss, Bundesrätin wurde und andererseits die Frauenrepräsentation in vielen Parlamenten substanziell stieg (genauer dazu: Fuchs 2015b). 
Integration von Gleichstellungsaspekten in die interne Weiterbildung (Interview). ${ }^{23}$

Im Zuge von Professionalisierungstendenzen der Personalarbeit in öffentlichen Verwaltungen seit den 1990er Jahren (Emery 2013) wurden Ende 1991 die „Weisungen über die Verbesserung der Vertretung und der beruflichen Stellung des weiblichen Personals in der allgemeinen Bundesverwaltung" erlassen. Weisungen sind ,generelle und abstrakte Anweisungen einer übergeordneten Behörde an ihr unterstellte Behörden, die hauptsächlich zum Zweck haben, eine einheitliche, gleichmäßige und sachgerechte Praxis zu gewährleisten“" (Mägli 2013: 127f.). Sie konkretisieren vorgelagerte Verordnungen, damit sie einheitlich ausgelegt und gehandhabt werden. Sie sind nur innerhalb der Behörden rechtlich bindend (ebd.: 128). De facto haben sie geringeres Gewicht und geringere Legitimität als Gesetze und Verordnungen. Die Weisungen von 1991 waren explizit als Frauenförderung formuliert und legten den Schwerpunkt auf Regeln und Empfehlungen zur Rekrutierung: So sollen Ausschreibungen geschlechtsneutral erfolgen und beide Geschlechter ansprechen; auch sollen Frauen bei gleicher Qualifikation bevorzugt berücksichtigt werden, solange sie in einem Amt unterrepräsentiert sind. Außerberufliche Qualifikationen seien bei der Bewertung einzubeziehen, dabei dürften beispielsweise Betreuungsaufgaben nicht geringer bewerten werden als Militärerfahrung (Pkt. 32). Die Weisungen postulieren einen verbesserten Zugang zur Weiterbildung für weibliche Beschäftigte und schreiben vor, bei Anfrage die Möglichkeit einer Teilzeitbeschäftigung zu prüfen. Zudem sollte die Bewertung und lohnmäßige Einreihung von Stellen auf ihre geschlechtsdiskriminierenden Wirkungen überprüft werden, vor allem die Frauenberufe (Pkt. 5). Schließlich werden Förderprogramme in der Verantwortung der Generalsekretariate der Departemente und der einzelnen Bundesämter gefordert, über deren Ergebnisse die Departemente zu unterrichten sind (Pkt. 9). Insofern waren die Weisungen sehr konkret, doch legten sie keinerlei Umsetzungsstrukturen fest und definierten keine konkreten Zielindikatoren. Die begrenzte Wirkung zeigt sich darin, dass 1997 erst gut die Hälfte der Einheiten in der Bundesverwaltung ein Förderprogramm etabliert hatte (Engeli 2011: 186f.).

Das neue Personalrecht des Bundes seit 2002 hat Kompetenzen vom Parlament zur Exekutive verschoben, d.h. sowohl zum Bundesrat als auch zu den Departementen oder gar den einzelnen untergeordneten Ämtern. In Tafel 4.1 sind die wichtigsten Grundsätze und Vorschriften zur betrieblichen Gleichstellung in den einzelnen Rechtsquellen aufgeführt.

23 Auch im Folgenden werden Erkenntnisse aus den Interviews lediglich mit dem Verweis „Interview(s)“ gekennzeichnet und keine weiteren Hinweise auf die interviewten Personen gegeben. 
Tafel 4.1: Rechtsgrundlagen betrieblicher Gleichstellungspolitik in der schweizerischen Bundesverwaltung

Bundespersonalgesetz BPG (SGS 172.220.1)
- Die Arbeitgeber müssen für Chancengleichheit und Gleichstellung von Frau und Mann geeig-
nete Maßnahmen treffen (Art. 4 Abs. 2d BPG);
- sie müssen Maßnahmen treffen, damit das Personal seine Verantwortung in Familie und
Gesellschaft wahrnehmen kann (Art. 4 Abs. 2i BPG) und
- sie sorgen für ein Beurteilungssystem der Mitarbeitenden, das Willkür im Arbeitsverhältnis ver-
hindert (Art. 4 Abs. 3 BPG).
Bundespersonalverordnung BPV (SGS 172.220.111.3)
- Der Arbeitgeber sorgt dafür, dass die Angestellten nicht aufgrund ihres Geschlechts oder ihrer
- Lebensform benachteiligt werden (Art. 6 Abs. 1 BPV).
- Die Departemente ergreifen geeignete Maßnahmen, um das Diskriminierungsverbot durchzu-
- setzen, darunter auch der Schutz vor sexueller Belästigung (Art. 6 Abs. 3 BPV).
- Die Departemente treffen gezielte Maßnahmen, um die Vertretung von Frauen in Kaderpositi-
Werhöhen (Art. 5 Abs. 2f. BPV).
Weisungen des Bundesrates zur Verwirklichung der Chancengleichheit von Frau und Mann in der
Bundesverwaltung (Novellierung 2003)
- Regelung der Zuständigkeiten innerhalb der Verwaltung;
- Pflicht zu einem Maßnahmenkatalog für vier Jahre;
- Vorgaben zu Personalgewinnung, -einsatz, -entwicklung und -beurteilung;
- Vorgaben zum Controllingprozess.
Verordnung über die Schlichtungskommission gemäß Gleichstellungsgesetz (SGS 172.327.1)
- Zusammensetzung, Organisation und Kompetenzen der Kommission bei Streitigkeiten nach
GIG (für die Beschäftigten freiwillig, für die Verwaltung obligatorisch

Quelle: eigene Zusammenstellung

Die 2003 neu formulierten Weisungen sind in der Sprache des New Public Management gehalten. Sie stellen die Förderung und Nutzung des ,weiblichen und männlichen Human- und Wissenspotenzials“ und die Stärkung von Konkurrenzfähigkeit und Attraktivität des Arbeitgebers Bundesverwaltung als zentrales Ziel dar. Gender Mainstreaming wird als Prinzip für den gesamten Prozess des Human Resource Managements bezeichnet (Pkt. 13). Die Departemente steuern die Politik, die Ämter setzen diese um. Chancengleichheit ist als zentrale Führungsaufgabe definiert, und es können Chancengleichheitsbeauftragte eingesetzt werden. Grundsätze und Regeln für Personalauswahl, -beurteilung und -entwicklung sind ausführlicher in Pkt. 4 zusammengefasst. Im Vergleich zu 1991 sind die Zuständigkeiten klarer geregelt: Die Departemente müssen messbare Zielvorgaben setzen, ein Vierjahresprogramm entwickeln und darüber jährlich berichten. Im Controlling werden Fortschritte und Zielerreichung regelmäßig überprüft, und es sollen ggf. Umlenkungsmaßnahmen ergriffen werden. Das Eidgenössische Personalamt erstellt jährlich einen Evaluationsbericht für den Bundesrat, der die wichtigsten Kennwerte enthält. (Pkt. 5). 2013 wurde das Controlling stark verein- 
facht. Neben den klareren Zuständigkeiten sind die Weisungen aber dennoch zu unkonkret, um eine „einheitliche, gleichmäßige und sachgerechte Praxis“ sicherzustellen. Welches ,die finanziellen und personellen Ressourcen“" sind, die für die tatsächliche Gleichstellung „notwendig“ sind (Pkt. 14) und wie dies bestimmt wird, wird ebenso wenig niedergelegt wie konkretisiert wird, wie denn ,[d]ie Chancengleichheit von Frau und Mann in das gesamte Human-Resources-Management auf allen Stufen, in alle Prozesse, insbesondere in die Führungsprozesse, sowie in alle Instrumente und Maßnahmen integriert" wird (Pkt. 13).

Zusammenfassend lässt sich festhalten: Das Personalrecht des Bundes arbeitet hauptsächlich mit Geboten und allgemeinen Zielformulierungen: Departemente und Ämter müssen die Chancengleichheit, die Vereinbarkeit von Beruf und gesellschaftlichem Engagement/Familie verfolgen, sie müssen das Diskriminierungsverbot durchsetzen und diskriminierungsfrei beurteilen. Mit den Weisungen wurden institutionelle Verfahren für Gleichstellungspläne und Controlling vorgeschrieben. Weitere institutionelle Vorkehrungen etwa für den Einsatz von Gleichstellungsbeauftragten, ihre Kompetenzen oder Ressourcen, gibt es nicht: Die Handlungsspielräume für die einzelnen Verwaltungseinheiten sind groß. Das Personalrecht macht zudem nur wenige Vorschriften, die auf substanzielle Politik zielen: So wird das allgemeine Ziel der Erhöhung des Frauenanteils in Kaderpositionen erwähnt. Die Verordnungen geben auch keine Vorschriften für Verfahren, wie diese Ziele angestrebt werden sollen, sondern erwähnen (unabgeschlossen) einige mögliche Methoden, wie Förderprogramme, Einsatz von Fachpersonen und Zielquoten. Diese Kombination von Konkretheit und Unverbindlichkeit hat heterogene Organisationsmuster und unterschiedliche Maßnahmenprofile in den untersuchten Bundesämtern zu Folge. Diese Organisierung stellt einen wichtigen Unterschied zur stärkeren Verrechtlichung in Deutschland und Österreich dar.

In den nächsten Abschnitten werden die institutionellen Instrumente analysiert, die innerhalb der Bundesverwaltung die Strukturen für das Handeln (gleichstellungspolitischer) AkteurInnen schaffen und die den Rahmen für konkrete substanzielle Maßnahmen z.B. zur Vereinbarkeit oder Karriereförderung bilden. Der Fokus liegt hier wie in den anderen Länderkapiteln auf den Vorgaben und den Spielräumen der Gleichstellungsbeauftragten und den Gleichstellungsplänen: Inwiefern determinieren diese institutionellen Regelungen in Bezug auf Verbindlichkeit, Reichweite und Ausdifferenzierung die (potenzielle) Effektivität betrieblicher Gleichstellungspolitik?

Wie im vorhergehenden Abschnitt zu den rechtlichen Grundlagen herausgearbeitet, gibt es für die schweizerische Bundesverwaltung keine Vorschriften zur Organisation der Gleichstellungsarbeit in den Einheiten oder zu den Ressourcen, sondern es werden nur übergeordnete Ziele formuliert. Für diese Ziele können bestimmte Verfahren und Maßnahmen eingesetzt werden, müssen es aber nicht (vgl. Tab. 4.1). Dieser Gestaltungsspielraum bietet das Po- 
tenzial, auf unterschiedliche Problemlagen einzugehen. Tatsächlich ist die Organisation der Gleichstellungspolitik in der Bundesverwaltung sehr heterogen, die Unterschiede sind dabei aber kaum sachlich begründet. Dabei ist betriebliche Gleichstellung im Departement des Äußeren am ausdifferenziertesten institutionalisiert. Die Koordination der Politik durch das Eidgenössische Personalamt ist relativ gering, was auch dem institutionellen Spannungsfeld zwischen Personalamt und eigenständigen Personalabteilungen in den Ämtern und Departementen geschuldet ist.

\subsubsection{Verantwortliche für die betriebliche Gleichstellung: Heterogene Institutionalisierung}

Eidgenössisches Personalamt

Innerhalb der Bundesverwaltung ist das Eidgenössische Personalamt (EPA) ein Dienstleistungszentrum für Human-Resource-Fragen, aber aus institutionellen Gründen nur in geringem Maße eine Koordinierungsinstanz für Gleichstellungsfragen. Das EPA ist als Kompetenzzentrum für die Konzeptualisierung und Implementation von Instrumenten, für Weiterbildung und Beratung in Personalbelangen zuständig. Personalabteilungen können auf diese Dienstleistungen zurückgreifen, betreiben aber auch ihre eigene Personalpolitik: Beispielsweise finden Rekrutierungsprozesse in den einzelnen Einheiten statt. Für Linienvorgesetzte ist die eigene Personalabteilung wesentlich wichtiger und generell sehr hilfreich. Im EPA ist für die Erarbeitung der Gleichstellungsziele (s.u.), für das regelmäßige Reporting zu den Gleichstellungszielen sowie für die Koordination der Umsetzung von Gleichstellungsmaßnahmen heute das „Diversity Management“ im Bereich des HumanResource-Marketing tätig. Dabei ist der Geschlechteraspekt der wichtigste Teilbereich neben der Förderung der Mehrsprachigkeit und der Förderung von Behinderten. Auf der anderen Seite sind die Departemente und Ämter für die Umsetzung und die Zielerreichung verantwortlich. Idealerweise arbeitet das EPA strategisch, und die Linienvorgesetzten sind operativ tätig. Die sog. HR-Konferenz, in der die Personalleitenden aus den Departementen und Ämtern zusammentreffen, soll die Koordination und konsistente Anwendung aller Regelungen sicherstellen.

Bis 2011 koordinierte und leitete das Eidgenössische Personalamt das sog. Forum, in dem alle Gleichstellungsbeauftragten der Ämter zusammenkamen, diskutierten und ihre Arbeit koordinieren konnten. Der Versuch, sich als interdepartementale Arbeitsgruppe mit einer eigenen Geschäftsordnung zu institutionalisieren, scheiterte am Widerstand der Direktion des EPA (Interview). Einige Interviewte äußerten sich kritisch zur tatsächlichen Leistung dieses Gremiums, zeigten aber auch Unverständnis darüber, dass ein solch großer Arbeitgeber wie die Bundesverwaltung fast keinen Austausch über 
Ideen, Ressourcen und Know-how in diesem Bereich hat. Tatsächlich ist es kaum möglich, in einer so großen Organisation ohne eine koordinierende institutionalisierte Arbeitsgruppe ein gemeinsames Verständnis des Gleichstellungsproblems und seiner möglichen Lösungen zu entwickeln (vgl. Jüngling/Rastetter 2011). Die Bedeutung der Diskussion und Auseinandersetzung für ein gemeinsames Verständnis wurde auch im vorhergehenden Beitrag am Beispiel der DEZA nochmals deutlich. Diese Situation schwächt daher die Implementation von Gleichstellungspolitik und eine mögliche Stärkung und Weiterentwicklung der Zielsetzungen für die gesamte Bundesverwaltung. Das Forum wurde 2012 offiziell abgeschafft, nachdem man seine „Zweckmäßigkeit“ geprüft hatte: „Neu werden in Koordination mit der HRK [Human-Resource-Konferenz, GF] unter der Federführung des EPA auftragsbezogen themenspezifische Arbeitsgruppen eingesetzt.“ (EPA 2012: 11f.) Hiernach wurde die Stabsstelle Chancengleichheit innerhalb des Außendepartements zusätzlich zum EPA Mitglied der Schweizerischen Konferenz der Gleichstellungsbeauftragten, des Vernetzungs- und Koordinationsgremiums staatlicher Gleichstellungsstellen (genauer dazu: Fuchs 2015b). Im Frühjahr 2014 wurde ein solches Gremium mit dem Fokus auf Informationsanlässe allerdings reaktiviert.

Die geringe Koordinierungsfunktion des EPA für betriebliche Gleichstellung ergibt sich aus mehreren Faktoren: Es gibt keine rechtlichen Vorgaben für eine Koordinierung, die über das Erstellen des Reportings hinausgeht, und es gibt einen systematisch bedingten Konflikt bzw. eine Konkurrenz zwischen zentralem Personalamt und ämterinternen Personalabteilungen bzw. den für Gleichstellungsmaßnahmen zuständigen departementalen Generalsekretariaten. Schließlich hängt innerhalb der Gesamtpolitik des Bundesrates der Stellenwert der Personalpolitik davon ab, wer dem Finanzdepartement vorsteht, in dem das EPA angesiedelt ist, so die Einschätzung einiger Fachpersonen. Zurzeit, mit der amtierenden Bundesrätin Eveline Widmer-Schlumpf, stünde Personalpolitik wieder stärker im Fokus, so einige der Interviewten.

Organisation in den einzelnen Ämtern: Verschiedene Arten von Gleichstellungsbeauftragten

Die Chancengleichheitsweisungen bestimmen, dass die Departemente mit einer Vierjahresplanung die Gleichstellungspolitik steuern und die Ämter diese Politik umsetzen (vgl. Abschnitt 4.3). Dies wird durch die Personalabteilungen wahrgenommen, die in den Generalsekretariaten angesiedelt sind, d.h. die Stabsstellen der jeweiligen Departemente. In einigen Fällen wird die Aufgabe der Chancengleichheit bei den zuständigen Personen direkt ausgewiesen, etwa im Departement des Inneren EDI, im Verteidigungsdepartement VBS oder im Department des Äußeren EDA; in anderen ist es nicht unmittelbar ersichtlich. Die Personalentwicklung innerhalb der Personalabteilungen ist im Bereich der betrieblichen Gleichstellung unterschiedlich stark aktiv. Im 
Volkswirtschaftsdepartement EVD/WBF, zu dem zwei der hier untersuchten Ämter gehören, sind jedoch umfassende Pakete mit verschiedenen Maßnahmen entwickelt worden (vgl. Abschnitt 4.4.1), die vor allem für die Beschäftigten gut sichtbar sind.

Innerhalb der Bundesverwaltung gibt es drei verschiedene Arten, die Umsetzung von Gleichstellungsmaßnahmen zu institutionalisieren. Im Departement für auswärtige Angelegenheiten EDA ist die Form Resultat einer Reorganisierung und ist einzigartig. Allerdings waren keine umfassenden Daten darüber verfügbar, wo die Arbeit wie organisiert ist und welche Organisationsform überwiegt. Tafel 4.2 fasst dabei die Eckdaten der Gleichstellungspolitik in den vier untersuchten Ämtern zusammen.

Im ersten Fall, dem Eidgenössischen Departement des Äußeren (EDA), besteht eine zentrale Stabsstelle, die im Generalsekretariat angesiedelt ist. Sie ist für die strategische Planung und Implementation „des gesamten Gleichstellungsprozesses" verantwortlich, d.h. Beratung, Sensibilisierung, Ausarbeitung innovativer Arbeitszeitmodelle und Förderung einer „Gleichstellungskultur". Diese Organisationsform ist wesentlich stärker als die anderen in Bezug auf Sichtbarkeit für die Beschäftigten, die eingesetzten Ressourcen und damit Maßnahmen und Kompetenzen (d.h. Verbindlichkeit). Die Stelle wurde und wird von Frauen geleitet, die vorher als kantonale Gleichstellungsbeauftragte gearbeitet haben und damit über langjährige, einschlägige und ausgesprochen breite Expertise verfügen. Die Stabsstelle unterstützt die Direktion und Linienvorgesetzte in deren Aufgaben. Die Stabsstelle präsidiert das „Sounding Board“ mit Mitgliedern aus verschiedenen Direktionen bzw. Ansprechpersonen in Auslandsbüros bzw. Botschaften (die auch als Focal Points bezeichnet werden) und ist verantwortlich für Ideenaustausch, Controlling und Reporting. So führt sie mit den Leitungen der einzelnen Direktionen regelmäßig Gespräche zu den Gleichstellungszielen und den dafür notwendigen Aktivitäten, womit ein gewisser Legitimationsdruck aufrechterhalten wird. Die Stabsstelle wurde erst 2008 während einer größeren Reorganisation des EDA geschaffen und ersetzte die verschiedenen Gleichstellungsbeauftragten in den Direktionen des EDA. Die Gleichstellungsbeauftragte der Direktion für Entwicklung und Zusammenarbeit DEZA (mit 60 Stellenprozenten), die immer eine stärkere Stellung als ihre KollegInnen in den anderen Direktionen des EDA hatte, übernahm die erste Leitung der Stabsstelle. 2012 hatte diese 2,5 Stellen und war auch verantwortlich für Mehrsprachigkeit und Integration von Behinderten. Die Gleichstellungsbeauftragten haben bisher beratend an Vorstellungsgesprächen teilgenommen (vgl. Abschnitt „Rekrutierung“, S. 91). In der DEZA erinnert man sich an die eigenen Gleichstellungsbeauftragten als starke Persönlichkeiten: „Die hat man gekannt, mit denen hat man sich besprochen, mit denen hat man sich auseinandergesetzt" (Interview). Dies unterstreicht, wie wichtig physische bzw. räumliche Präsenz dafür ist, das Thema auf der internen Agenda zu 
Tafel 4.2: Organisation der Gleichstellungsarbeit in der schweizerischen Bundesverwaltung

Eidgenössisches Personalamt

Stabsstelle Diversity Management (110 Stellenprozente): Beratung und Unterstützung; Monitoring der Personalentwicklung; federführend bei Konzeption der Personalstrategie des Bundesrats inkl.

Bereithaltung einzelner Handreichungen (Musterreglement sexuelle Belästigung, Wegleitung

Telearbeit etc.); Vernetzung in der Schweizerischen Konferenz der Gleichstellungsbeauftragten

\begin{tabular}{|c|c|c|}
\hline \multirow[b]{2}{*}{$\begin{array}{l}\text { Stabsstelle Chancengleichheit } \\
\text { im EDA }\end{array}$} & \multicolumn{2}{|c|}{$\begin{array}{l}\text { Chancengleichheitsverantwortliche in den Generalsekreta- } \\
\text { riaten: } \\
\text { Strategische und konzeptionelle Arbeit mit Zielen und Maß- } \\
\text { nahmen für das gesamte Departement }\end{array}$} \\
\hline & $\begin{array}{l}\text { Chancengleichheitsbeauf- } \\
\text { tragte in den Ämtern }\end{array}$ & $\begin{array}{l}\text { Chancengleichheit als Aufgabe } \\
\text { der Personalkommission }\end{array}$ \\
\hline $\begin{array}{l}\text { Legitimation: Ernennung } \\
\text { durch Amtsleitung, unbefristet }\end{array}$ & $\begin{array}{l}\text { Legitimation: Ernennung } \\
\text { durch Amtsleitung, unbefris- } \\
\text { tet }\end{array}$ & $\begin{array}{l}\text { Legitimation: Mitarbeitende } \\
\text { wählen für ihre Abteilung } \\
\text { Mitglieder der PK; teilw. Er- } \\
\text { nennung durch Abteilung }\end{array}$ \\
\hline $\begin{array}{l}\text { Aufgaben } \\
\text { - Geschlecht, Mehrsprachig- } \\
\text { keit, Behinderung } \\
\text { - Bedarfe \& Ist-Zustand } \\
\text { erheben, Policies erarbeiten } \\
\text { und umsetzen „in the entire } \\
\text { gender equality process“ } \\
\text { - Besonders: Erhöhung } \\
\text { Frauenanteil; ,workplace } \\
\text { policies“; Prävention sexuel- } \\
\text { ler Belästigung, auch Bera- } \\
\text { tung von Einzelpersonen } \\
\text { - Beratung der Leitung ein- } \\
\text { zelner Direktionen } \\
\text { - Beisitz bei Rekrutierungen/ } \\
\text { Rotationsgesprächen mögl. } \\
\text { - Unterstützung durch } \\
\text { "Sounding Board“ mit er- } \\
\text { nannten Mitgliedern }\end{array}$ & $\begin{array}{l}\text { Aufgaben } \\
\text { - In der Regel Geschlecht, } \\
\text { Mehrsprachigkeit, Behin- } \\
\text { derung } \\
\text { - Themen im Bereich } \\
\text { aufgreifen und Projekte } \\
\text { initiieren } \\
\text { - Beratung und Unterstüt- } \\
\text { zung der Amtsleitung, z.B. } \\
\text { beim Controlling } \\
\text { - Anlaufstelle für Mitarbei- } \\
\text { tende } \\
\text { - Unterstützung durch } \\
\text { freiwillige Arbeitsgruppe } \\
\text { oder Personalkommission } \\
\text { möglich }\end{array}$ & $\begin{array}{l}\text { Aufgaben } \\
\text { - Information und Mitsprache } \\
\text { bei Personalfragen } \\
\text { - auch bei Chancengleichheit } \\
\text { (Geschlecht, Mehrsprachig- } \\
\text { keit) } \\
\text { - Anlaufstelle für Fragen der } \\
\text { Mitarbeitenden }\end{array}$ \\
\hline $\begin{array}{l}\text { Kompetenzen } \\
\text { Interne Informationstätigkeit, } \\
\text { interne Veranstaltungen, } \\
\text { Zugang zu Direktionen }\end{array}$ & $\begin{array}{l}\text { Kompetenzen } \\
\text { Interne Informationstätigkeit, } \\
\text { interne Veranstaltungen, } \\
\text { Zugang zur Amtsleitung }\end{array}$ & $\begin{array}{l}\text { Kompetenzen: } \\
\text { Interne Informationstätigkeit, } \\
\text { interne Veranstaltungen, } \\
\text { Zugang zur Amtsleitung }\end{array}$ \\
\hline $\begin{array}{l}\text { Ressourcen: } 250 \text { Stellenpro- } \\
\text { zente }\end{array}$ & $\begin{array}{l}\text { Ressourcen: typischerweise } \\
\text { 10-20 Stellenprozente }\end{array}$ & $\begin{array}{l}\text { Ressourcen: keine feste } \\
\text { Regelung }\end{array}$ \\
\hline $\begin{array}{l}\text { Vernetzung }{ }^{\star}: \text { Mitglied der } \\
\text { Schweizerischen Konferenz } \\
\text { der Gleichstellungsbeauftrag- } \\
\text { ten }\end{array}$ & Vernetzung*: Keine & $\begin{array}{l}\text { Vernetzung*: Personalkom- } \\
\text { missionen aller Ämter in } \\
\text { Koordinationskommission } \\
\text { vernetzt }\end{array}$ \\
\hline
\end{tabular}

* Vernetzung bis 2011 im „Forum“

Quelle: eigene Zusammenstellung 
halten, und dies kann natürlich nur mit entsprechenden Ressourcen geschehen. Die meisten in der DEZA Interviewten beurteilen die Umstrukturierung kritisch im Vergleich zur Situation vor 2008, als die Gleichstellungsbeauftragte auch physisch präsent war und das Thema viel weiter oben auf der Agenda, denn: „Aus den Augen, aus dem Sinn.“ Einige wünschen sich, dass die Stabsstelle bestimmte Themen proaktiv bearbeitet (vgl. Abschnitt 7.5 in diesem Buch).

Aktuell erarbeitet die Stelle Handreichungen zur Gleichstellung, die Linienvorgesetzte einsetzen können, und diskutiert sie mit ihnen. ${ }^{24}$ Zum Zeitpunkt der Interviews 2012 war die Entwicklung sog. Workplace Policies fast abgeschlossen; ausgehend von den Kooperationsbüros der DEZA wurden solche Leitbilder der Zusammenarbeit vom lokalen und entsandten Personal vor Ort erarbeitet. In einem aufwändigen Diskussionsprozess mit den Ansprechpersonen der Focal Points hat die Stabsstelle einen Mindeststandard zu Themen wie Diskriminierung, sexuelle Belästigung, (kulturelle) Vielfalt, Mobbing und geschlechtergerechte Rekrutierung von Personal erarbeitet Themen, die weder lokales Recht noch das Bundespersonalrecht berühren, sondern eine gemeinsame Wertebasis formulieren.

Zweitens sind in den Ämtern einzelne Beschäftigte für Gleichstellungsfragen zuständig, wobei ein gewisser kleiner Prozentsatz ihrer Stelle dafür vorgesehen wurde (z.B. 20\%). Diese Beauftragten haben vor allem beratende und initiierende Funktion, jedoch keine Kontroll- oder Einsprachekompetenzen; Ziele der betrieblichen Gleichstellung aus den Departementen werden von der Amtsleitung abgeleitet und konkretisiert. Die Beauftragten werden von der Amtsleitung ernannt und kooperieren mit der Leitung sowie der Personalabteilung. Die Personalkommission (s.u.) oder ein Gruppe von Freiwilligen kann deren Arbeit unterstützen. Von den untersuchten Ämtern ist nur noch das Bundesamt für Landwirtschaft auf diese Weise organisiert. Dort hat die Beauftragte beispielsweise ein Zeitbudget von 10\% sowie einen nicht näher benannten finanziellen Etat. Sie soll Chancengleichheit zwischen Frau und Mann, verschiedenen Sprachgruppen und Berufsgattungen sowie zwischen jungen und älteren Mitarbeitenden anstreben. $\mathrm{Zu}$ den Aufgaben und Kompetenzen gehören die Beratung und Unterstützung von Leitung, Vorgesetzten und Mitarbeitenden, das Aufgreifen von Themen und Projekten, die Unterstützung beim Chancengleichheitscontrolling des Amtes sowie die Vernetzung mit anderen Ämtern. Die Beauftragte hat dabei eine „,beratende und unterstützende Funktion" und kann Anliegen in der Geschäftsleitung vertreten, welche die Entscheidungsbefugnis hat (Bundesamt für Landwirtschaft 2010).

24 Persönliche Mitteilung Regula Kuhn, 10. September 2014. 
Es ist unklar, wie viele Ämter noch nach diesem Modell verfahren..$^{25}$ Der Gleichstellungsbericht 2004-2007 stellte eine große Variationsbreite bei diesen Positionen in den verschiedenen Departementen fest und verdeutlichte auch große Probleme mit dieser Organisationsform: So fehlten häufig Motivation und Engagement der Beauftragten. Die relativ große Fluktuation führte zum Verlust von Expertise, und die Übernahme des Amtes habe sogar manchmal eine negative Wirkung auf die Leistungsbeurteilung (EPA 2009: 11f.). Im Staatssekretariat für Wirtschaft SECO traten fünf Gleichstellungsbeauftragte vor einigen Jahren unter Protest zurück, weil sie mit ihren Anliegen kein Gehör fanden und sich in ihrer Arbeit permanent blockiert sahen. Ironischerweise sind ihre damaligen Forderungen - Telearbeit, flexible Arbeitszeiten, Teilzeit - heutzutage Standardinstrumente der betrieblichen Gleichstellungspolitik (Interviews). Diese Organisationsform ist wesentlicher schwächer als die erste Form der zentralen Stabsstelle - in Bezug auf Sichtbarkeit, die Ressourcen und Kompetenzen. Die Beauftragten haben im Wesentlichen Unterstützungs- und Artikulationsfunktion. Dabei liegt die Etablierung und Umsetzung von Maßnahmen in den Händen der Amtsleitung. Es fehlen Durchsetzungskompetenzen - in Konfliktfällen wie oben erwähnt können Beauftragte fast nichts tun oder in letzter Konsequenz nur das Amt zur Verfügung stellen.

Fluktuation und fehlende Motivation dürften mit Gründe für die dritte Organisationsform sein. Dort übernimmt die Personalkommission Aufgaben betrieblicher Gleichstellungspolitik als Personalpolitik. Von den untersuchten Ämtern haben das Staatssekretariat für Wirtschaft SECO und die Finanzverwaltung EFV die Aufgaben der Gleichstellung ihren Personalkommissionen übergeben. Letztere sind beratende Gremien, die die Sichtweise, die Anliegen und Ideen der Beschäftigten einbringen sollen. Personalkommissionen sind in der Bundespersonalverordnung erwähnt (Art. 109 BPV), doch hat jede Kommission ihre eigene Geschäftsordnung. Mit der deutschen oder österreichischen Mitbestimmung in Personal- und Betriebsräten sind die Personalkommissionen nicht $\mathrm{zu}$ vergleichen. ${ }^{26}$ Diese Übertragung mutet zwar zunächst sinnvoll an, doch werden offenbar Gleichstellungsthemen nur selten eingebracht und sind nicht besonders wichtig in der alltäglichen Arbeit (Interviews). Die Sichtbarkeit betrieblicher Gleichstellung ist in dieser Form am geringsten, und personelle wie finanzielle Ressourcen sind nicht ausgewiesen

25 So ist im Anhang des Berichts zur Chancengleichheit von $2012 \mathrm{zu}$ lesen, alle Einheiten im Verteidigungs- sowie im Finanzdepartement hätten Beauftragte für Chancengleichheit, womit zumindest in der Finanzverwaltung dann auch die Personalkommissionen mitgemeint wären.

26 In etwa der Hälfte der Ämter in der Bundesverwaltung bestehen Personalkommissionen, die ihrerseits wieder in einer Koordinationskommission vernetzt sind. Eine Institutionalisierung dieses Gremiums mit einer Geschäftsordnung konnte aber gegen den Willen der EPAAmtsleitung nicht durchgesetzt werden. Nach Aussage des Präsidenten dieser Koordinationskommission gibt es kaum mehr Kandidierende als Sitze in den Personalkommissionen. 
und gering. Aufgefangen werden kann dies zumindest zum Teil durch Aktivitäten des Departements: Im Volkswirtschaftsdepartement EVD/WBF unterstützt eine starke Personalentwicklungsabteilung im Generalsekretariat die Gleichstellungsbeauftragten und die Personalkommissionen. So stellte diese Abteilung 2004 ein umfassendes Konzept für die Förderung der Gleichstellung vor und wurde mit Erarbeitung und Umsetzung des „Programms Leuthard" 2006/07 beauftragt (vgl. dazu Abschnitt 4.4.1).

Innerhalb der Bundesverwaltung ist die Wichtigkeit einer starken Institutionalisierung, von personenunabhängigen Prozeduren und Regeln, für die betriebliche Gleichstellung umstritten, auch unter den Chancengleichheitsbeauftragten, wie diese Zitate exemplarisch zeigen:

„Man kann Regeln und Gesetze haben, aber wenn man keine Struktur zur Verfügung stellt und keine finanziellen und personellen Ressourcen um die Gleichstellung tatsächlich zu erreichen, dann ist das eine ,mission impossible'."

Andere betonen die Wichtigkeit individueller Lösungen und persönlichen Engagements:

„Ich denke, es ist ein guter Weg, dass man die individuellen Probleme wahrnimmt und auch versucht, sie individuell zu lösen. Es ist oftmals ein Problem, dass man schöne allgemeingültige Richtlinien hat, die aber Theorie bleiben und nicht umgesetzt werden. Das ist der weniger gute Weg. Wir haben keine Richtlinien, leben es dafür. Es braucht aufgeschlossene Chefs auch in der Personalabteilung, Leute, die selber Kinder haben und das aus eigener Erfahrung kennen, dass sie das nachvollziehen können.“

Die hier festgestellte Unterschiedlichkeit der Organisationsformen ist durch die offene Formulierung in den rechtlichen Grundlagen angelegt. Die Heterogenität scheint aber im Wesentlichen situativ bedingt: Es konnten keine sachlichen Gründe für unterschiedliche Organisationsformen gefunden werden, die sich aus den unterschiedlichen Aufgaben oder Spezifika eines Amtes ergeben würden. Einzig im EDA mit seinen vielen Außenstellen erscheint eine Zentralisierung der Gleichstellungsarbeit sinnvoll, da die geografische Streuung und kulturelle Diversität groß ist. Der politische Wille der Bundesrätin zum Zeitpunkt der Reorganisation war ebenfalls groß. Will man eine einheitliche Praxis betrieblicher Gleichstellung - und damit auch den gleichen Zugang für alle Beschäftigten zu entsprechenden Maßnahmen -, ist eine solche Heterogenität hinderlich und eine stärkere Zentralisierung in den Departementen sinnvoll. Mehr personelle und finanzielle Ressourcen bzw. deren Bündelung ermöglichen auch mehr Maßnahmen. Schließlich ist die Sichtbarkeit des Ziels Chancengleichheit für die Beschäftigten wichtig. Dabei schneidet die dritte Form, die Integration in die Personalkommissionen, am schlechtesten ab: Chancengleichheit löst sich gleichsam als eigenes Thema auf. Andererseits ermöglichen es Personalkommissionen, Arbeitsgruppen für Chancengleichheit auf Ämterstufe oder ein „Sounding Board“, den Puls der Belegschaft zu fühlen: Diese Möglichkeit ist in allen drei Formen gegeben. 
Schließlich ist ohne Koordination und Austausch über Ziele und geeignete Vorgehensweisen eine gemeinsame kohärente betriebliche Politik nicht möglich. Dies gilt umso mehr für die Gleichstellung der Geschlechter, da dies eine transformatorische Aufgabe ist, die standardmäßig auf Widerstände stößt.

\subsubsection{Gleichstellungspläne: Auf dem Weg zum Gleichstellungscontrolling?}

Die Ziele betrieblicher Gleichstellung sind in der Bundesverwaltung in die allgemeine Personalstrategie und das allgemeine Controlling integriert. Gleichzeitig werden spezifische Reportings und Evaluationsberichte erstellt. Neben dieser Stärke besteht jedoch die Gefahr, dass aufgrund von quantitativen „Zielbändern“ und fehlenden Konsequenzen bei Nichterreichung wenig ambitiöse Ziele und kaum qualitative Ziele formuliert werden.

Das EPA ist das federführende Amt bei der Erarbeitung der Personalstrategie des Bundes, die vom Bundesrat verabschiedet wird. Ein solcher Entwurf geht in einem mehrstufigen Verfahren in die Ämterkonsultation und das Mitberichtsverfahren bei allen Departementen (vgl. Klöti et al. 2014: 208210). Die Chancengleichheitsbeauftragten können im Rahmen dieser Konsultation über ihr Departement Stellung nehmen, sind aber selbst in der Regel nicht an der konzeptionellen Entwicklung beteiligt, sondern nur die Personalabteilungen in den Generalsekretariaten der Departemente. Der Bundesrat verabschiedet schließlich diese Strategie. Er erhält vom Personalamt jährlich Berichte über die Entwicklung im Personalwesen, die als jährliches „Reporting Personalmanagement" an die Geschäftsprüfungskommission und die Finanzkommission der Eidgenössischen Räte gehen. Neben diesem Bericht erstellt das Personalamt mithilfe der Daten aus den Departementen auch den jährlichen Bericht zur Chancengleichheit, der neben Geschlechtergleichstellung auch die Mehrsprachigkeit und die Integration von Behinderten abdeckt. Hierbei sind auch nach Geschlecht aufgeschlüsselte Daten über Löhne, Leistungsbeurteilungen und Kaderpositionen zu finden. Alle vier Jahre wird ein längerer Evaluationsbericht erarbeitet (vgl. EPA 2002, 2004, 2009, 2012).

In der Personalstrategie sind gleichstellungspolitische Zielsetzungen in einem eigenen Kapitel integriert („Chancen der Vielfalt nutzen“, vgl. EPA 2010: 23). Dabei sind quantitative Ziele für die Gleichstellung der Geschlechter aktuell nicht mehr mit einem Zielwert bezeichnet, sondern mit Zielbändern - z.B. ein Anteil von Frauen im mittleren Management zwischen 29\% und 34\% (vgl. EPA 2012: 9; Stand 2011: 23,5\%, vgl. Abb. 4.3, S. 65). Für die Grenzwache und die Verteidigung liegen die Zielbänder beim Frauenanteil niedriger: Der Frauenanteil soll ohne Verteidigung insgesamt zwischen $44 \%$ und $48 \%$ liegen, beim Militär zwischen $11 \%$ und 12\% (Stand 2013: 
12,5\%) und bei der Grenzwache 8,5\% bis 10,5\% (Stand 2013: 9\%; vgl. EPA 2014: 9). Das Anknüpfen an den Ist-Zustand ist sinnvoll; ob Zielbänder Flexibilität geben oder das Anpeilen des unteren Werts begünstigen, lässt sich noch nicht abschließend beurteilen. ${ }^{27}$

In den Departementen und Ämtern werden auf Grundlage der Personalstrategie und der Zielwerte/Zielbänder spezifische angepasste Maßnahmen geplant, von der Leitung verabschiedet und umgesetzt. Auch hier sind die Chancengleichheitsbeauftragten beratend tätig. Grundsätzlich liegt die Erreichung der Ziele wie bei anderen Themen auch in der Verantwortung von Linie und Personalabteilung; für die Information und die Sensibilisierung sind auch die Beauftragten zuständig. Jährlich werden Fortschrittsberichte erstellt, die intern zugänglich sind. Solche Berichte sind in der Regel ausführlich (bis zu 20 Seiten) und enthalten Kennzahlen, durchgeführte Maßnahmen und Empfehlungen. Aufgrund der jährlichen Berichterstattung des EPA werden nötige Anpassungen in den Maßnahmen in der HR-Konferenz diskutiert, an der die PersonalleiterInnen aller Ämter teilnehmen.

Die Integration der Gleichstellungsziele in die allgemeine Personalstrategie trägt Züge des Gleichstellungscontrollings zumindest im Personalbereich $^{28}$ und ist im Sinne des Gender Mainstreaming positiv zu bewerten. Gleichstellungscontrolling bedeutet, dass die Gleichstellungsziele einer Organisation in die routinemäßigen Planungs- und Steuerungsprozesse integriert werden. Maßnahmen eines Gleichstellungscontrollings im gesamten personalwirtschaftlichen Zyklus (Ist-Analyse, Rekrutierung und Nachwuchsförderung, Personalentwicklung) und zu Vereinbarkeit, partnerschaftlichem Verhalten am Arbeitsplatz, zur Institutionalisierung von Chancengleichheitsarbeit sowie die ,programmatische Grundsatzerklärung“ sind in der Bundesverwaltung vorhanden (vgl. Müller/Sander 2005: 58f.).

Gleichzeitig besteht die Gefahr, dass die Zielsetzungen im Aushandlungsprozess von Departementen, EPA und schließlich Bundesrat wenig ambitiös ausfallen, auch um den erwartbaren Widerstand aus den Departementen geringer zu halten. In den Interviews wurde verschiedentlich kritisiert, die Zahlen seien so gesetzt, dass sie „niemandem wehtun“ und leicht erreicht werden könnten. Auch fehlen Sanktionen, wenn Ziele nicht erreicht werden (Zollinger/Fuchs 2011: 13-16). Um diese Schwächen zu kompensieren, kann nach Einschätzung von Personalentwicklern in den Departementen ein internes Benchmarking helfen, weil sich „hinterherhinkende“ Einheiten verpflichtet fühlen, aktiver zu werden. Enthält dieses Benchmarking auch Aussagen über qualitative Ziele und gute Maßnahmen, lässt sich auch eine

27 Die offiziellen Daten der Geschlechterverteilung sind stets ohne den Bereich Verteidigung berechnet. Das ist irritierend, macht aber einen positiven Eindruck. Es erschwert die Vergleichbarkeit aktueller Daten.

28 Gleichstellungscontrolling kann ebenso für die Leistungen/Produkte der Verwaltung oder des Unternehmens durchgeführt werden. 
Fixierung auf Zahlen überwinden. Es bleibt jedoch festzuhalten, dass ein solches Benchmarking nicht institutionell für die gesamte Bundesverwaltung abgesichert ist, sondern vom Engagement der departementalen Personalabteilungen abhängt.

\subsubsection{Zwischenfazit: Institutionalisierung lediglich als Teil der Personalpolitik}

Inwiefern strukturieren nun die hier besprochenen institutionellen Regeln die Effektivität betrieblicher Gleichstellungspolitik vor? Betriebliche Gleichstellungspolitik ist stark in die allgemeine Personalpolitik integriert, was die quantitativen Ziele betrifft. Sie ist aber kaum als eigenständiges Handlungsfeld institutionalisiert. Departemente und Ämter haben breiten Handlungsspielraum, was die Organisation der Gleichstellungsarbeit angeht. Für die jeweilig unterschiedlichen Organisationsformen der betrieblichen Chancengleichheit sind kaum sachliche Gründe erkennbar. Die Strategie der minimalen Rahmenregelung führt also weniger zu angepassten Lösungen, als wenn die Einheiten es machen können, wie es sich ergibt. Die Verantwortung für betriebliche Gleichstellung ist zwischen Organisationsstufen geteilt - der Bundesrat gibt strategische Ziele vor, die Departemente transformieren sie für die unterstellten Ämter; die Ämter, aber auch die Departemente, sollen konkrete Maßnahmen entwickeln und umsetzen. Die Chancengleichheitsbeauftragten der einzelnen Ämter sind nicht vernetzt. Auf der Ebene der Bundesverwaltung sind sie auch nicht konzeptionell-strategisch an der Entwicklung von Zielen beteiligt. Die stärkste Institutionalisierung und Sichtbarkeit hat das Departement des Äußeren mit seiner zentralen Stabsstelle, die schwächste Form ist jene der Personalkommissionen. Im EDA ist dadurch auch das meiste Fachwissen konzentriert. Geringere Ressourcen, wenig Sichtbarkeit und die starke Integration in die Personalpolitik resultieren nicht in einem Gender Mainstreaming, sondern in einem Aufgehen bzw. Versickern der Gleichstellungsfragen vor allem in Personalfragen. Durch eine fehlende starke Vernetzung und Kooperation ist die Entwicklung von gemeinsamen Problemverständnissen und geteilten Visionen nicht möglich (vgl. dazu auch das siebte Kapitel in diesem Buch). Für die Umsetzung konkreter Maßnahmen sind daher erhebliche Unterschiede zwischen den Ämtern zu erwarten. 


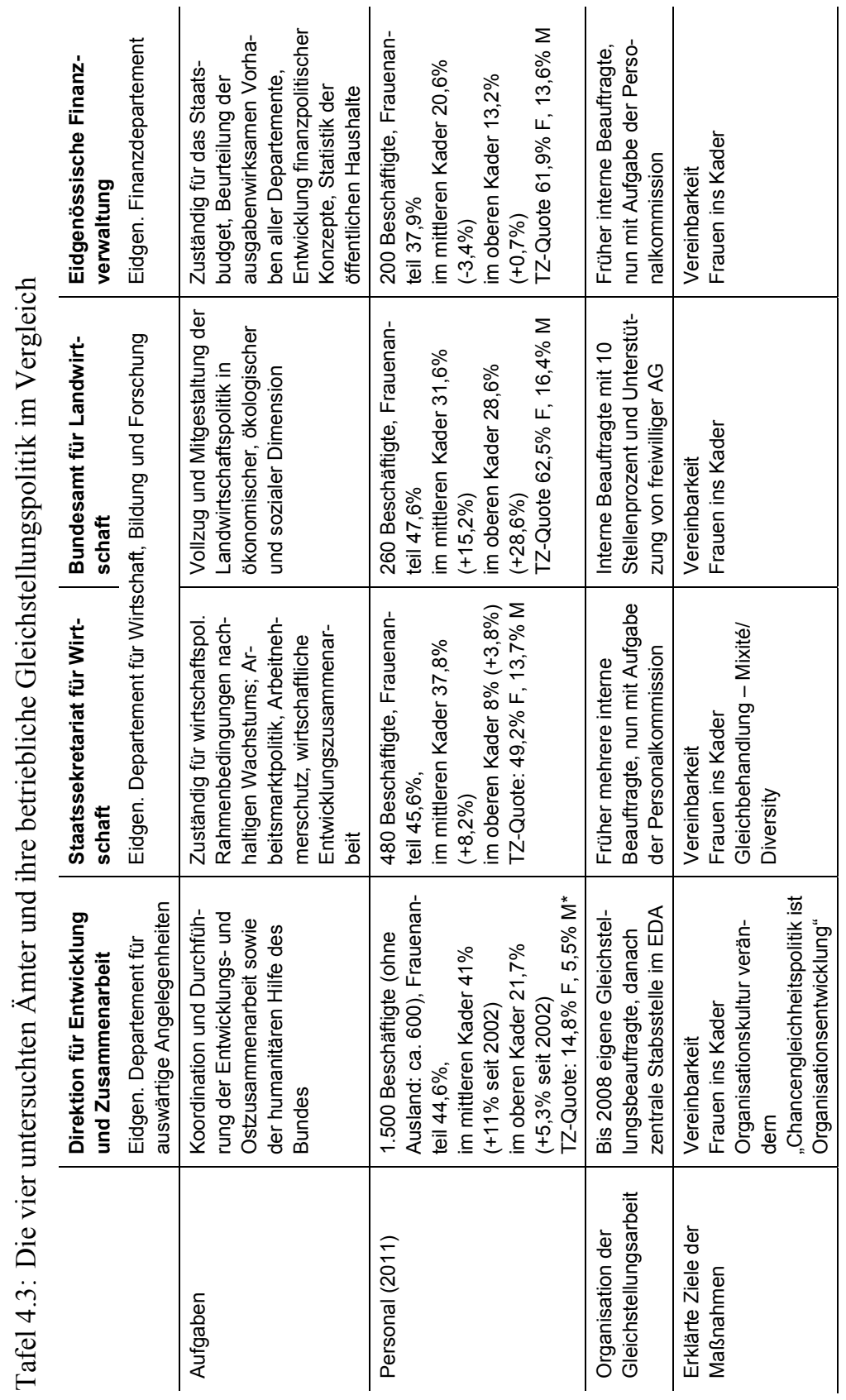




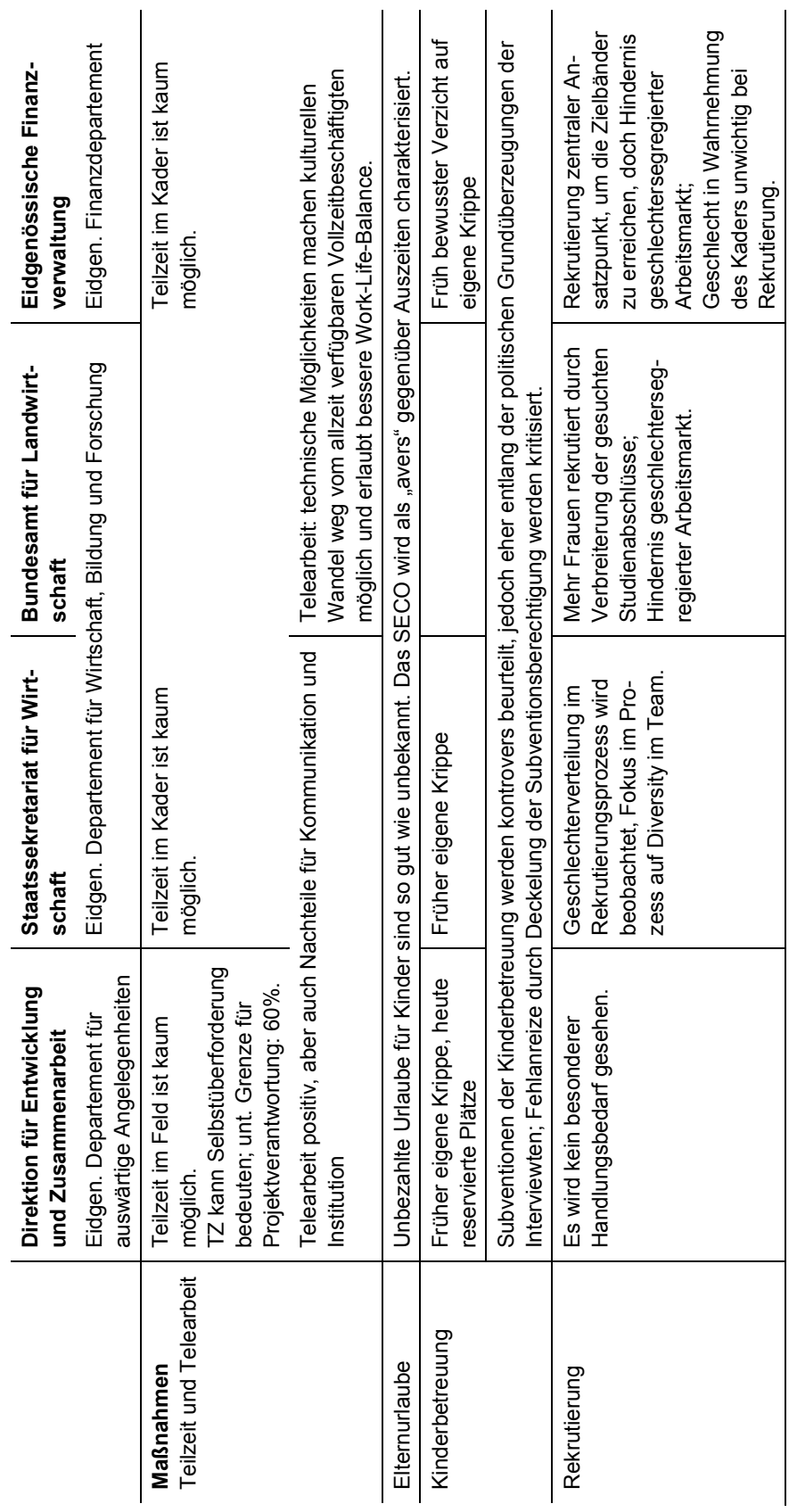




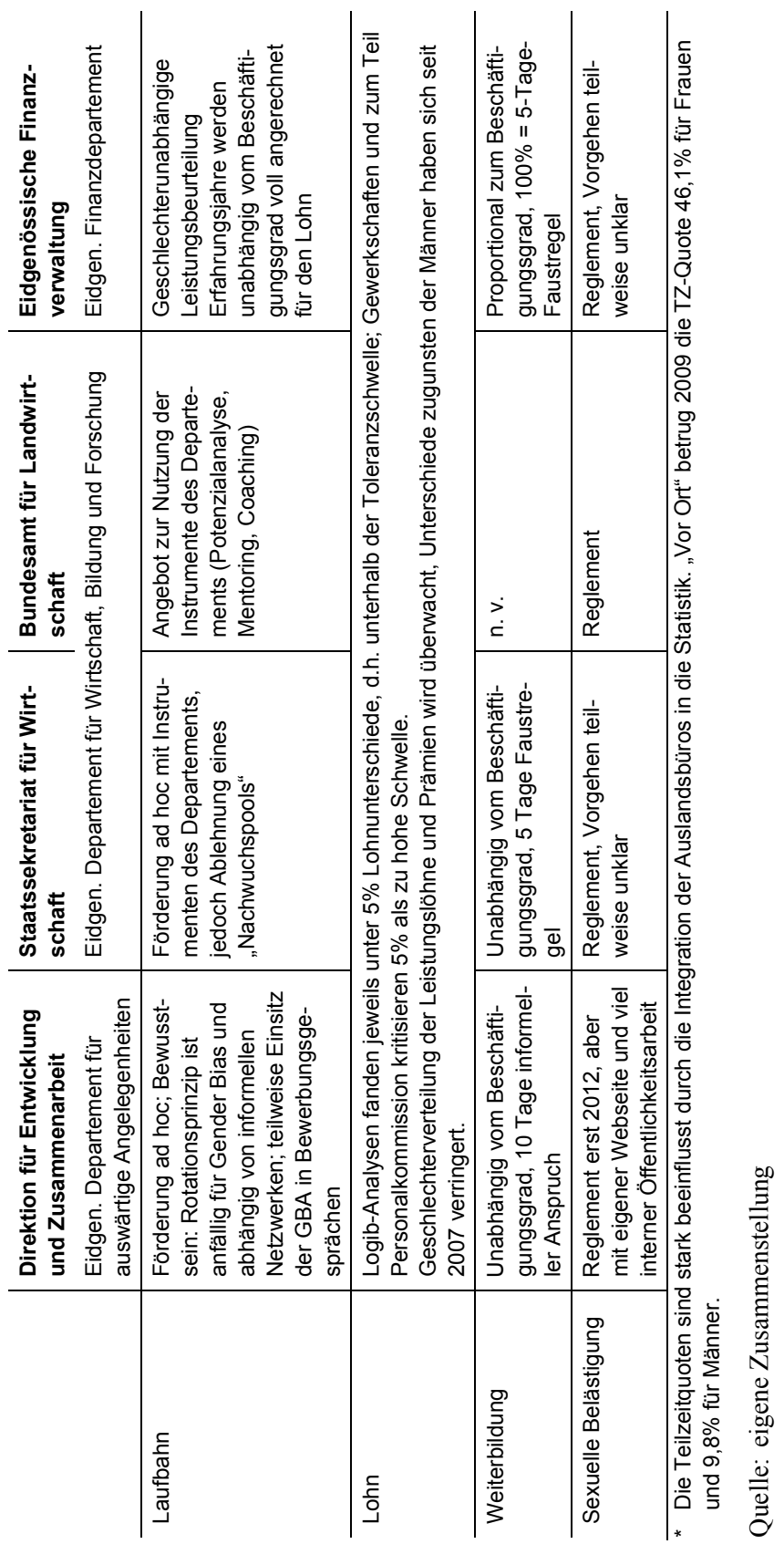




\subsection{Substanzielle Politik in der schweizerischen Bundesverwaltung}

Der folgende Abschnitt befasst sich nun mit den einzelnen konkreten Maßnahmen auf der Ebene der Beschäftigten und des Verwaltungsalltags. Wesenskern und Ziel betrieblicher Gleichstellungspolitik, wie sie in den Weisungen, Berichten, Strategien und in den Interviews aufscheinen, sind die Vereinbarkeit von Familie und Beruf sowie ein höherer Anteil von Frauen in Führungspositionen. Alle Maßnahmen lassen sich im Prinzip diesen zwei Zielen - Vereinbarkeit und Frauen in Führungspositionen - zuordnen. Allerdings sind auch Programme entwickelt worden mit dem Ziel, verschiedenartige Maßnahmen zusammenwirken zu lassen; sie werden hier zuerst behandelt.

\subsubsection{Programme: Umfassend und à la carte}

Langjährige strategische Programme zur Chancengleichheit mit programmatischen Grundsatzerklärungen wie in der Direktion für Entwicklung und Zusammenarbeit (DEZA 1997) und im Volkwirtschaftsdepartement (EVD 2004), die Maßnahmen zur Vereinbarkeit und Karriereförderung miteinander kombinieren, können auf langfristige und substanzielle Erfolge blicken: So ist der Anstieg des Frauenanteils im mittleren und oberen Kader zwischen 2006 und 2011 im EVD der zweitstärkste bzw. stärkste der gesamten Bundesverwaltung (vgl. Abb. 4.3 und 4.4). Im Fall der DEZA leiten sich diese Programme aus der inhaltlichen Arbeit ab, die u.a. Geschlechtergerechtigkeit zum Ziel hat (vgl. dazu genauer Kapitel 7 in diesem Buch), im Fall der Programme im Volkswirtschaftsdepartement ist ein starker politischer Willen der verantwortlichen Bundesrätin erkennbar, der von der verantwortlichen Personalabteilung verstärkt wird und dessen Maßnahmen zudem heute auf die gesamte Bundesverwaltung ausgedehnt sind.

In der DEZA wurde 1997 ein Programm verschiedet, das langfristige Ziele bis 2010 festlegte. Es forderte eine betriebliche Gleichstellungspolitik und eine entsprechende Beauftragte analog zur Umsetzung der Genderpolitik in der Entwicklungszusammenarbeit. Das Programm wurde aus einer Organisationsentwicklungs-Perspektive geschrieben und bezieht daher Organisationsstruktur und -kultur mit ein. Die aktuelle Strategie des Departements für auswärtige Angelegenheiten ist ähnlich langfristig angelegt (2010-2020; EDA 2011). Im Kontext des Aufbaus einer professionellen Personalentwicklung verabschiedete das EVD 2004 ein Leitbild zu Chancengleichheit (EVD 2004). Es formuliert die Grundsätze von Chancengleichheit als Querschnittsund Führungsaufgabe und als Qualitätsmerkmal einer nachhaltigen Personalpolitik; geschlechtsspezifische Maßnahmen dienen der Verwirklichung der 
tatsächlichen Gleichstellung (ebd.: 3). Die zentralen Ziele sind die Vereinbarkeit von Beruf und Privatleben, die Erhöhung des Frauenanteils auf allen Ebenen und gleiche Chancen bei Rekrutierung und Aufstieg. Dazu sind Teilzeit- und Telearbeit aufzubauen und Co-Leitungen auf Kaderstufe zu entwickeln. Zudem werden Laufbahnentwicklungskurse für Frauen angeboten. Im Leitbild sind die Maßnahmen konsequent mit einzelnen Abschnitten der Chancengleichheitsweisungen verknüpft; die Auswahl der Maßnahmen steht den einzelnen Ämtern jedoch frei. Das Leitbild sieht ein quantitatives und qualitatives Reporting vor. Die Berichte sind departementsintern sichtbar und wirken als Benchmark: Das quantitative Reporting zu Lohnklassen, Leistungsbeurteilungen und Prämienvergabe zeigte anfangs große Geschlechterunterschiede, die sich schlagartig nach den ersten Veröffentlichungen verminderten (Interview). Das qualitative Reporting muss alle zwei Jahre von den Führungskräften mit mehr als zehn Mitarbeitenden ausgefüllt werden, „die sich mindestens eine halbe Stunde mit Chancengleichheit auseinandersetzen" und ihre Maßnahmen und Erfahrungen beschreiben müssen. Es wurde zudem ein Preis für das beste Projekt in diesen Reportings ausgeschrieben. Strategisch ging ein Preis ans Bundesamt für Landwirtschaft, dessen Direktor Chancengleichheit als eines der fünf Amtsziele definiert hatte. Der Preis erzeugte in der Folge hohen Handlungsdruck auf die Rekrutierung im oberen Kader - dies ist gewissermaßen die "hidden agenda“ dieser Maßnahme. In kurzer Zeit stieg der Frauenanteil im BLW von $0 \%$ auf $28,3 \%$ in den obersten Lohnklassen.

Die neue gewählte Bundesrätin Doris Leuthard (CVP) wollte im Volkswirtschaftsdepartement 2006 mit gutem Beispiel vorangehen und ,ein Zeichen setzen“. Deswegen wurde das Leitbild mit einem „Konzept Vereinbarkeit von Familie und Beruf" ergänzt und ausgeweitet (EVD 2007). Es beinhaltete zusätzlich ein Anrecht auf Reduzierung der Arbeitszeit, Förderung flexibler Arbeitszeiten und Telearbeit, Betreuungssubventionen, umfassende Beratung zur Kinderbetreuung und fünf statt zwei Tagen bezahlte Vaterschaftsurlaub. Besonders dieser Urlaub löste ein großes Medienecho aus, denn die Bundespersonalverordnung sah damals erst zwei Tage vor. Der zusätzliche Urlaub konnte deswegen vorderhand nicht gewährt werden, trotzdem konnte Bundesrätin Leuthard mit allen anderen vernünftigen Maßnahmen ein Zeichen setzen. 2008 entwickelte das Generalsekretariat das Instrument der individuellen „Potenzialanalyse“ als kleines Assessment, um vor allem Frauen zu ermutigen, sich auf Kaderstellen zu bewerben. In einer Nachbefragung gaben zwei Drittel der Teilnehmerinnen an, sie seien einen Karriereschritt weiter (Interview).

Im EVD stieg der Anteil von Frauen im oberen Kader zwischen 2006 und 2011 von $8 \%$ auf 18\%. Die einzelnen Instrumente mit der Bandbreite von Vereinbarkeit bis Laufbahn verstärken sich gegenseitig, so die Einschätzung von Fachpersonen: So bewirken bewusste Rekrutierungsbemühungen und 
individuelle Potenzialanalyse der weiblichen Beschäftigten zusammen einen Anstieg qualifizierter Bewerbungen von Frauen auf Kaderstellen, die sich in einem transparenten Prozess dann auch öfter durchsetzen; zusätzlicher Schub in diesem Bereich dürfte zudem der politische Wille der Bundesrätin entfaltet haben. Insgesamt entwickeln Programme daher mehr Schubkraft als vereinzelte Maßnahmen.

\subsubsection{Maßnahmen zur Vereinbarkeit von Familie und Beruf: Langsamer Abschied von der Anwesenheitskultur}

Arbeitszeit, Freistellungsregelungen und Kinderbetreuung sind zentrale Handlungsfelder für eine bessere Vereinbarkeit von Familie und Beruf. Während durch die steigende Teilzeit- und Telearbeit ein klarer Kulturwandel weg vom stets präsenten Vollzeitarbeiter zu konstatieren ist, sind (unbezahlte) Urlaube für Kinderbetreuung unüblich und kaum kulturell akzeptiert. Die Unterstützung der Kinderbetreuung hat sich von vereinzelten eigenen Krippen hin zur Subvention der Betreuungskosten verschoben, deren konkrete Anwendung als einzige aller Maßnahmen durch die Einführung von Einkommensgrenzen als Fehlanreiz gesehen werden kann.

\section{Arbeitszeit}

Arbeitszeitarrangements wurden in Dokumenten und in den Interviews oft als Herzstück effektiver Gleichstellungsmaßnahmen beschrieben. Teilzeitarbeit ist recht verbreitet und steigt langsam für beide Geschlechter an, vor allem in den unteren Lohnklassen. In der gesamten Bundesverwaltung lag sie 2013 bei 49,2\% für Frauen und 10,6\% für Männer (zum Vergleich: 2004 47,2\% für Frauen und 7,3\% für Männer). ${ }^{29}$ Die befragten ExpertInnen konstatierten in der Bundesverwaltung einen wichtigen kulturellen Wandel in den letzten Jahren: Heute könne fast jeder und jede Teilzeit arbeiten, wenn auch mit relativ großem Stundenvolumen. Das geschlechtsspezifische Muster der Arbeitszeiten hat sich jedoch nirgendwo verändert. In der Praxis sorgen Leitfäden von EPA und Departementen für ein geregeltes Vorgehen beim Wunsch nach Teilzeitarbeit, seit 2013 haben junge Eltern einen Rechtsanspruch auf Arbeitszeitreduktion.

Trotz des Anstiegs der Teilzeitarbeit für beide Geschlechter haben sich die geschlechtsspezifischen Muster nicht verändert: Insgesamt steigt die Teilzeitquote von Männern nicht stärker als die der Frauen. Im Bewusstsein der Interviewten sind jedoch Beispiele von Vätern sehr präsent, die Teilzeit arbeiten möchten; sie konzedieren dabei, dass der kulturelle Wandel nicht vollkommen ist: Es brauche immer noch Mut für junge Väter, wenn sie ihre 
Arbeitszeit reduzieren wollen. Allerdings gibt es auch Dominoeffekte in Teams oder Departementen (Interviews). Hingegen ist Teilzeitarbeit in höheren Führungspositionen immer noch eine Herausforderung und nicht allgemein akzeptiert (Interviews). Dabei ist schon heute die „große Teilzeit“ ab $70 \%$ weit verbreitet. Ihr Anteil liegt in den untersuchten Ämtern zwischen $47 \%$ (EFV) und 58\% (BLW) aller Teilzeitbeschäftigungen. ${ }^{30}$

Bis 2013 gab es keinerlei Ansprüche auf Teilzeitarbeit. Seit Juli 2013 haben Eltern allerdings bei Geburt/Adoption eines Kindes einen Anspruch auf Reduktion des Beschäftigungsgrads um höchstens $20 \%$, wobei der Beschäftigungsgrad dabei nicht unter 60\% fallen darf (Art. 60a BPV). Ein Recht auf Wiederaufstockung gibt es jedoch nicht. Wie mit den „eingesparten“ Stellenprozenten verfahren wird und wem sie gutgeschrieben werden (dem Team, der Abteilung, dem Amt), ist komplex und offenbar nicht überall gleich geregelt. Diese Regelung kann jedoch als qualitativer Sprung bezeichnet werden: Zwar gibt das Personalamt konkrete Anleitungen für die Prüfung von Telearbeit, Teilzeitarbeit und Jobsharing, ${ }^{31}$ doch verbriefte Ansprüche sind ein Novum. ${ }^{32}$

In der öffentlichen Diskussion, namentlich in der Deutschschweiz, wird Teilzeitarbeit oft als ein Allheilmittel für mehr Gleichstellung im Erwerbsleben propagiert, und problematische Aspekte davon werden ausgeblendet (etwa Nachteile bei Einkommen, Altersversorgung und Laufbahnaussichten). Die Fachpersonen in der Bundesverwaltung betrachteten Teilzeitarbeit differenziert: Teilzeitarbeit wird mehrheitlich wohlwollend, aber mit kritischen Untertönen bewertet. So erwähnten einige eine untere Grenze von $60 \%$ als notwendig für konzeptionelle und Programmarbeit. Zudem sei Teilzeit eine soziale und institutionelle Herausforderung für Teams, denn es braucht ein hohes $\mathrm{Ma} ß$ an Koordination und Flexibilität der Beteiligten. Die informellen Gesprächssituationen nähmen ab und der Teamgeist könne darunter leiden. In der DEZA waren die kritischsten Stimmen zu finden (vgl. das siebte Kapitel in diesem Buch); gegenüber dem Glauben an die Machbarkeit von Doppelkarriere und (hoher) Teilzeit mit Kindern wurden mehrmals die Grenzen persönlicher Leistungsfähigkeit und die Grenzen der Institution angesprochen.

Auch die Telearbeit nimmt zu, d.h. die Erledigung von Aufgaben an einem Arbeitsplatz zu Hause. Dazu werden leider keine zentralen Statistiken geführt. In den Augen der Interviewten stellt Telearbeit von zu Hause aus mit

30 Eigene Berechnungen nach EPA-Datensatz für 2011. Über alle Departemente hinweg schwankt dieser Anteil zwischen knapp 30\% im Justizdepartement und 62\% im Umweltund Verkehrsdepartement.

31 „Richtlinien zur Telearbeit in der Bundesverwaltung“, Ms., November 2007. Gewerkschaften bewerteten solche Richtlinien als eher vage und abhängig vom konkreten Vorgesetzten. Sie sind in der Beratung mit jenen seltenen Fällen konfrontiert, in denen keine Einigung erzielt werden kann oder Vorgesetzte Teilzeitarbeit rundheraus ablehnen.

32 Es gibt noch keine Zahlen dazu, wie stark dieser Anspruch genutzt wird. 
einem Laptop und einer gesicherten Verbindung ein wesentliches und unentbehrliches Instrument für eine gute Work-Life-Balance dar. In den drei Ämtern außer der DEZA wurde die Überzeugung geäußert, der technologische Fortschritt habe entscheidend zu einer besseren Akzeptanz von solchen flexiblen Arrangements beigetragen. ${ }^{33}$ Allerdings brauche Telearbeit Vertrauen zwischen Beschäftigten und Vorgesetzten, Selbstdisziplin und Engagement auf beiden Seiten. Explizit wandte sich ein Vorgesetzter gegen die Auffassung in seinem Amt, Telearbeit sei nur für Mütter mit kleinen Kindern möglich. Mehrmals sprachen Vorgesetzte explizit ihre Fürsorgepflicht an, also im Blick zu haben, ob sich die Mitarbeitenden überlasten oder ihre Arbeitszeit sich zunehmend entgrenzt und in die Freizeit übergreift. In der DEZA ist Telearbeit auch anzutreffen; hier wird sie als hervorragend für Konzeptarbeit und als negativ für soziale Kontakte und den (inhaltlichen) Austausch im Team beurteilt.

Jobsharing ist nach Aussage von Personalverantwortlichen nicht sehr weit verbreitet, da es sehr anspruchsvoll hinsichtlich Arbeitsinhalt und Zusammenarbeit der Beschäftigten ist. Die in den Interviews erwähnten Beispiele sind dennoch sehr erfolgreich. Jobsharing wird in Programmdokumenten und Berichten zur Gleichstellung immer erwähnt, wobei dies möglicherweise vor allem erfolgt, um sich als attraktiver Arbeitgeber darzustellen (Interview). Mögliche Spannungen zwischen Teilzeitarbeit und Karriereverlauf wurden nicht angesprochen.

\section{Elternurlaube}

Im Vergleich zu Deutschland und Österreich erscheinen die Ansprüche und Regelungen im Zusammenhang mit Kindern relativ bescheiden, im Vergleich zur Schweizer Privatwirtschaft sind sie relativ großzügig, wenn auch nicht immer großzügiger als die von Großunternehmen (vgl. Fuchs 2008a). Die Möglichkeit unbezahlter Freistellungen wird offenbar kaum genutzt. Inwiefern es immer noch zu Kündigungen seitens junger Mütter kommt oder inwieweit sich kontinuierliche Erwerbstätigkeit als neue Norm durchsetzt - ob es also gleichstellungspolitische Probleme gibt -, kann nicht beurteilt werden, weil entsprechende Zahlen nicht erhoben werden.

In der Bundesverwaltung gibt es 16 Wochen voll bezahlten Mutterschaftsurlaub (Art. 60 BPV) und seit Juli 2013 zehn Tage bezahlten Vaterschaftsurlaub (vorher waren es fünf Tage; vgl. Art. 40 para 3 lit b VBPV).

33 Hier fällt ins Gewicht, dass in der Schweiz sehr viel gependelt wird und dies wegen des beruflichen Engagements von Paaren und steigender Wohnungspreise zunimmt. Für die Bundesverwaltung kommt hinzu, dass gerade WestschweizerInnen aus französischsprachigen Orten nach Bern hineinpendeln und ein Tag Telearbeit leicht zwei Stunden Freizeit mehr pro Tag bedeuten kann. Zwei Drittel der Erwerbstätigen arbeiten nicht in ihrer Wohngemeinde. 10\% der Erwerbstätigen haben einen Arbeitsweg von mehr als einer Stunde (vgl. Bundesamt 2013: 5). 
Weitergehende unbezahlte Urlaube sind zwar in Artikel 61 BPV erwähnt, aber sie sind in der Praxis unüblich und für Väter unbekannt; allenfalls eine Ausdehnung des Mutterschaftsurlaubs auf ein halbes Jahr kommt vor. Mehrmals wurde in den Interviews die Kultur im eigenen Amt als ,avers“ gegenüber Pausen oder Sabbaticals bezeichnet. Nach Aussage der interviewten Personalverantwortlichen gibt es immer weniger „Wiedereinsteigerinnen“, welche die idealtypischen Stufen Berufstätigkeit - Kinderpause - Wiedereinstieg durchlaufen. Eine solche Pause wird, auch von Vorgesetzten, zunehmend als Problem wahrgenommen (Interviews). Leider gibt es zu den bezahlten und unbezahlten Urlauben keine Daten. Möglicherweise etabliert sich hier im Zusammenwirken mit den kurzen gesetzlich bezahlten Zeiten eine neue Norm der kontinuierlichen Erwerbstätigkeit für Mütter, die schnell nach 16 bzw. höchstens 26 Wochen Urlaub - wieder in den Job zurückkehren, aber dabei ihre Arbeitszeit reduzieren. Der Bericht einer Expertin, sie sei vor einigen Jahren die Erste gewesen, die in ihrer Abteilung Mutterschaftsurlaub genommen habe und dieser sei arbeitsorganisatorisch nicht richtig vorbereitet gewesen, deutet darauf hin, dass kontinuierliche Erwerbstätigkeit von Müttern ein relativ neues Phänomen ist.

Es ist eine offene Frage, ob in einem intensiven „Fokus auf Wiedereinsteigerinnen" wie in Deutschland und Österreich oder in der kontinuierlichen Erwerbstätigkeit von Müttern ein größeres Potenzial für die berufliche Gleichstellung liegt.

\section{Kinderbetreuung}

Die Unterstützung der Kinderbetreuung für Beschäftigte in der Bundesverwaltung nimmt verschiedene Formen an. Heute besteht sie im Wesentlichen aus dem Subventionsregime der Betreuungskosten, wie es im „Programm Leuthard" niedergelegt war und wie es heute für die gesamte Bundesverwaltung gilt; im Volkswirtschaftsdepartement kommt noch ein externer Beratungsservice hinzu. Diese Subvention hat als einzige Maßnahme Fehlanreize durch die politisch begründete Einkommensgrenze für die Ansprüche.

Einige Ämter betrieben eigene Krippen, wie etwas das SECO oder die DEZA. Heute sind diese Einrichtungen nicht mehr exklusiv, weil Kinderbetreuung sinnvollerweise in Wohnortnähe stattfindet; allerdings bleiben vereinzelt noch Plätze reserviert. Im EVD wurde die Idee entwickelt und umgesetzt, für die Suche und Beratung nach geeigneter Betreuung einen professionellen externen Anbieter zu beauftragen, nämlich Childcare Service Schweiz. Im EVD gab es seit 2008 Subventionen zwischen 50\% und 100\% der Betreuungskosten für Vorschulkinder ungeachtet des Haushaltseinkommens. Seit 2011 sind diese Subventionen für alle Bundesbeschäftigten zugänglich, allerdings ist das Haushaltseinkommen bei 20.000 Franken pro Monat plafoniert. Das Grundprinzip und die Idee gehen bis in die 1990er Jahre zurück, als es erstmals ein kleines Budget für solche Zuschüsse im Eidgenössischen Perso- 
nalamt gab (Interview). Die tatsächliche Subvention wird in einer eher komplizierten Prozedur errechnet. Es gibt Konstellationen, in denen die Annahme der Subvention in de facto niedrigerem Haushaltseinkommen und/oder höheren Steuern resultiert (Interview).

Während alle ExpertInnen die Gleichstellungsmaßnahmen in der Bundesverwaltung für konsistent halten und einer gemeinsamen Philosophie verpflichtet sehen, wird die Subvention quer durch alle Ämter kontrovers beurteilt. Für die einen ist es Fehlanreiz: Es sei ungerecht, die Subvention bei 20.000 Franken Haushaltseinkommen zu plafonieren, da viele Familien mit zwei erwerbstätigen HochschulabsolventInnen leicht mehr verdienten, sodass die Deckelung zusammen mit externen Fehlanreizen die Erwerbstätigkeit von Frauen unattraktiver mache. Einige sehen die Subvention als sog. „fringe benefit" und die Einkommensgrenze als eine politische motivierte Grenze („Wir machen nicht Sozialpolitik, sondern Personalpolitik!“). Andere hingegen beurteilten die Regelung als sehr großzügig oder äußerten grundsätzliche ordnungspolitische Bedenken. ${ }^{34}$ Subventionen scheinen von allen vorkommenden Instrumenten besonders anfällig für inkonsistente Anreize zu sein. Mehr als andere interagieren sie mit externen Regelungen in der Sozial-, Steuer- oder Familienpolitik. Ob der Fehlanreiz bei gut qualifizierten Mitarbeitenden tatsächlich wirkt und ob er wesentlich ist, ist nicht genau festzustellen; vermutlich ist er eher klein.

\subsubsection{Maßnahmen zur Karriereförderung von Frauen: Geringe Verbindlichkeit}

Die Rekrutierung und Laufbahnförderung ist ein zweiter zentraler Bereich, der in den Weisungen angesprochen wird. In der großen Bandbreite der Maßnahmen ist nur eine Maßnahme, die Ausschreibung der Stellen mit dem Zusatz „80-100\%“", verbindlich (Weisungen Pkt. 413). Die Maßnahmen für mehr Frauen in der Bundesverwaltung sind überwiegend Kann-Maßnahmen, von denen meistens unbekannt ist, wie stark und von wem sie genutzt werden. Nur vereinzelt gibt es interne Evaluationen (z.B. Potenzialanalyse für Frauen, siehe Abschnitt 4.4.1). Klar personalwirtschaftliche Maßnahmen etwa zur Rekrutierung, zu den Prämien oder der Leistungsbeurteilung sind bei den Personalfachleuten gut verankert. Nur das EDA verfügt über die Möglichkeiten und Ressourcen, dass Gleichstellungsfachleute den Rekrutie-

34 Zwei ExpertInnen äußerten grundsätzliche Bedenken: Kinderbetreuung sei nicht Aufgabe des Arbeitgebers; Subventionen oder Steuervorteile gehören aus Gründen der Subsidiarität und des Föderalismus auf die kantonale und kommunale Ebene. Gewerkschaften finden die Prozedur kompliziert und vermuten, dass viele Beschäftigte mit der Annahme der Subvention schlechter dastehen; ein Fall lag zum Zeitpunkt des Interviews bei einem von der Gewerkschaft beauftragten Anwalt. 
rungsprozess beobachten. Ein stärkerer Einsatz der Maßnahmen tendiert zu einem stärkeren Anstieg von Frauen in Kaderpositionen (vgl. Abschnitt 4.4.1).

\section{Rekrutierung}

Die zentrale Bedeutung einer gleichstellungsgerechten Rekrutierung für den Zugang von Frauen in ein Unternehmen (vgl. Kay 2011) spiegelt sich in ausführlichen Regeln in den Chancengleichheitsweisungen von 1991 und 2003. So sollen die Personalwerbung besonders BewerberInnen des bisher untervertretenen Geschlechts berücksichtigen, das untervertretene Geschlecht bei gleichwertiger Qualifikation vorrangig berücksichtigt werden, im Bewerbungsverfahren Fachpersonen beiderlei Geschlechts mitwirken und dort außerberufliche Erfahrungen (z.B. Betreuungsarbeit) berücksichtigt werden. Ein militärischer Grad darf nur dort verlangt oder berücksichtigt werden, wo entsprechende Fachkenntnisse nachweislich unerlässlich sind (Pkt. 41 und 42 der Weisungen).

Die konkrete Auswahlpraxis kann hier nicht beurteilt werden. Die Ansprache von Frauen und Männern in Stellenanzeigen erfolgt unterschiedlich, insbesondere wird die 80-100\%-Regel in den Stellenanzeigen der Tagespresse sehr unterschiedlich befolgt. ${ }^{35}$ Einige Ämter verfolgen die Geschlechterverteilung bei Bewerbungen, Einladungen und den tatsächlichen Anstellungen (wie das SECO). Angesichts einer starken horizontalen beruflichen Geschlechtersegregation gibt es in einigen Bereichen Schwierigkeiten, mehr Frauen anzustellen, weil keine oder nur sehr wenige Bewerbungen für eher technische Arbeitsplätze und Berufe eingehen. ${ }^{36}$

Ein starker politischer Wille und Handlungsdruck, wie in Abschnitt 4.4.1 am Beispiel des Bundesamts für Landwirtschaft erwähnt, kann zusammen mit der bewussten Ausweitung der gesuchten akademischen Qualifikationen (es werden nicht nur AgronomInnen, sondern auch BiologInnen und GeografInnen gesucht) zur Rekrutierung von hoch qualifizierten Frauen für das mittlere und obere Management führen.

Formale Quotenregelungen existieren nicht, ${ }^{37}$ obwohl die ehemalige AuBenministerin Micheline Calmy-Rey eine 50:50-Regel bei der Rekrutierung

35 Vereinzelte Äußerungen in den Interviews deuten darauf hin, dass den BewerberInnen in Vorstellungsgesprächen doch nahegelegt wird, $100 \%$ zu arbeiten.

36 Im Sample gilt das besonders für die Finanzverwaltung. Die Auswertung der Personaldaten der Gesamtbundesverwaltung hat auch gezeigt, dass es in männerdominierten Bereichen (Zoll, Grenzwache, Informatikabteilungen) in den letzten Jahren nicht gelungen ist, Frauen anzusprechen, und umgekehrt, dass Ämter mit einer Frauenmehrheit sich weiter feminisiert haben (z.B. Personalamt, Bundesamt für Kultur; eigene Berechnungen nach EPA-Datensatz).

37 Ein Manager erinnerte sich an die sehr effektive „Zwei-Haufen-Regel“, als er Mitte der 1990er Jahre für Ruth Dreifuss (erste sozialdemokratische Bundesrätin) arbeitete: auf den ersten kamen alle Bewerbungen von Frauen (und vielleicht Westschweizern), und nur wenn 
zukünftiger BotschafterInnen beim sog. Concours anwendete (Interview). Die Gleichstellungsbeauftragte des EDA nimmt aktuell als Beobachterin am Concours teil. ${ }^{38}$

Insgesamt sind mögliche Diskriminierungen im Auswahlprozess im Bewusstsein der Vorgesetzten und Personalfachleute kaum präsent, vielmehr spielt die Wahrnehmung eines ausgetrockneten Arbeitsmarkts eine große Rolle, dem man mit attraktiven Bedingungen wie z.B. Telearbeit oder „fringe benefits" für Hochqualifizierte entgegenkommen muss.

\section{Laufbahnentwicklung}

Coaching, Mentoring, Potenzialanalyse für Frauen sind in der Bundesverwaltung unterschiedlich verbreitet; spezifische Kurse für weibliche Führungskräfte des Personalamts stellen einen stabilen Teil des Weiterbildungsprogramms dar. Von systematischen Evaluationen dieser Instrumente ist nichts bekannt. In den 1990er Jahren wurde mit dem Personalamt ein Projekt zur Qualifizierung in frauentypischen „Einbahn-Berufen“ wie Sekretärin entwickelt (vgl. Stalder/Wägli 1995). Heute sind vor allem hoch qualifizierte Beschäftigte Zielgruppe von Laufbahnmaßnahmen, was zur Elitenfokussierung der betrieblichen Gleichstellung maßgeblich beiträgt. Einige Kurse und Beratungsinstrumente wurden als Frauenfördermaßnahmen entwickelt und wurden allmählich zu allgemeinen Maßnahmen für Nachwuchskräfte, wie etwa Coaching und Mentoring im Volkswirtschaftsdepartement. Die Ämter wenden diese Instrumente unterschiedlich und verschieden häufig an. Personalverantwortliche lehnten unisono die Idee eines „Karrierepools“ für aufstiegswillige Beschäftigte als kontraproduktiv ab. Einige waren auch recht skeptisch gegenüber der Potenzialanalyse des EVD/WBF. ${ }^{39}$

Die Leistungsbeurteilung der Mitarbeitenden war Ende der 1990er Jahren in einem Projekt unter Gleichstellungsgesichtspunkten überarbeitet worden, um auch Schlüsselqualifikationen zu erfassen, die z.B. bei Betreuungsarbeit erworben worden sind (vgl. Kadishi 2001). Es ist unklar, inwieweit das Instrument weiterentwickelt und eingesetzt wird. Der Beurteilungsprozess erfolgt aber überall nach klaren Verfahren. Zumindest in der Finanzverwaltung erfolgt bei Neueinstellungen die Einstufung innerhalb der Lohnklassen in die Erfahrungsstufen unabhängig vom vorherigen Beschäftigungsgrad. Die Geschlechterunterschiede bei Prämien und in der Leistungsbeurteilung sind zurückgegangen, seitdem es ein (grobes) veröffentlichtes und (feineres) internes Benchmarking gibt (vgl. Abschnitt 4.4.1). Diese Ergebnisse sind insgesamt ermutigend, aber es überrascht, dass Diskriminierung und Gleichstel-

deren Begutachtung keine guten Resultate ergab, wurden Bewerbungen von Männern in Betracht gezogen (Interview).

38 Persönliche Mitteilung Regula Kuhn, 10. September 2014.

$39 \mathrm{Zu}$ Laufbahnfragen in der DEZA vgl. Abschnitt 7.5 in diesem Buch. 
lung in der Leistungsbeurteilung Nicht-Themen sind, obwohl sie als wichtiger und entscheidender Faktor in der Literatur und in den anderen Länderberichten beschreiben werden (Krell 2011).

Schließlich haben alle untersuchten Ämter festgelegte Vorgehensweisen und Reglements für die Prävention von und den Umgang mit sexueller Belästigung, die bekanntlich berufliches Fortkommen und Arbeitszufriedenheit stark behindern kann und die im Gleichstellungsgesetz als Diskriminierung aufgrund des Geschlechts gilt. Diese Regeln werden auf dem Intranet publiziert und von Zeit zu Zeit, etwa im Mitarbeitenden-Newsletter, hervorgehoben. Im EDA trat ein entsprechendes Reglement erst 2012 in Kraft, was man auf ,,institutionelle Widerstände“ zurückführen könne. ${ }^{40}$ Die Bundesverwaltung erfüllt also ihre Präventionspflicht nach dem Gleichstellungsgesetz. Bekannt gewordene Fälle sexueller Belästigung sind jedoch auch hier selten. Ein Personalleiter erfuhr erst anlässlich eines „Austrittsgesprächs“ von entsprechenden Vorfällen, die dann auch zur Kündigung seitens der Mitarbeiterin geführt hatten. Nicht alle Personalfachleute und Vorgesetzte schienen im Gespräch darüber hinaus mit den etablierten Vorgehensweisen vertraut. Aktuelle Forschungsergebnisse zur Schweiz relativeren allerdings den Stellenwert von Reglements gegenüber anderen organisationalen Einflussfaktoren wie sexualisiertes Arbeitsklima, männliche Dominanz im Arbeitsalltag oder eine respektvolle und ethische Unternehmenskultur (Krings et al. 2013: 8)

\section{Lohngleichheit}

Im Nachgang zur großen Evaluation des Gleichstellungsgesetzes 2005 waren von den vielen Verbesserungsvorschlägen nur freiwillige Maßnahmen politisch mehrheitsfähig gewesen. Dazu gehörte auch der trilaterale sog. Lohngleichheitsdialog. Arbeitgeber- und Arbeitnehmervertretungen sowie der Bund bildeten eine Kommission, in der Arbeitgeber- und Arbeitnehmervertretungen freiwillig gemeinsam die Löhne mit dem Instrument Logib überprüften. Signifikante Lohnunterschiede, die über einer 5\%-Toleranzschwelle lagen, sollten innerhalb von vier Jahren beseitigt werde. Es war geplant, zwischen 2009 und 2013100 Unternehmen dafür zu gewinnen. Ende 2013 hatten 18 Firmen das Verfahren abgeschlossen und 23 waren noch im Verfahren. In der Presse galt der Dialog als gescheitert (näher dazu: Fuchs 2015b). In ihrer Vorbildfunktion beteiligte sich die Bundesverwaltung seit 2010 am Dialog. Die Lohnunterschiede blieben, abgesehen von Einzelfällen, unterhalb der Toleranzschwelle. Gewerkschaften kritisierten die Toleranz von 5\% als willkürlich und zu hoch angesetzt. Eine analytische Arbeitsplatzbewertung, die eruiert, ob gleichwertige Arbeiten auch gleich eingestuft sind (oder etwa

40 Das EDA verbreitete die Informationen zum neuen Reglement aber sehr breit mit Faltblättern und einer Webseite, die umfassende Informationen und Ratschläge enthält (siehe www. eda.admin.ch/respect). 
frauentypische Arbeiten zu niedrig bewertet werden), gab es aber in der Bundesverwaltung bisher nicht. Lohngleichheitsprozesse, wie sie bei kantonalen Arbeitgebern relativ häufig vorkamen (Fuchs 2013; vgl. http://gleichstellungs gesetz.ch), gab es im Bund jedoch ebenfalls nicht: Er wird als fairer Arbeitgeber wahrgenommen.

\subsection{Fazit: Große institutionelle Handlungsspielräume, schwache Koordination}

Inwiefern zeigt sich in der Bundesverwaltung ein angemessenes Steuerungsregime verschiedener Maßnahmen mit guten Wirkungsvoraussetzungen hinsichtlich Verbindlichkeit, Ausdifferenziertheit und Reichweite, das in sich konsistent ist und zudem kohärent mit weiterer Gleichstellungspolitik und gesellschaftlichen Werten?

In der Bundesverwaltung gibt es langsame, aber stetige Erfolge beim Frauenanteil an der Gesamtbeschäftigung und in Führungspositionen. Es ist ein kultureller Wandel weg vom allzeit anwesenden und verfügbaren Vollzeitarbeiter zu beobachten. Dies wird vor allem durch flexible Arbeitszeitarrangements und Teilzeit erreicht, in geringerem Maße auch durch besondere Förderung weiblicher Karrieren. Die geringe Verbindlichkeit der rechtlichen Grundlagen, der große Handlungsspielraum der Departemente und Ämter und das Fehlen von Freistellungsregelungen sind kohärent mit den liberalen Elementen des schweizerischen Geschlechterregimes (vgl. 4.1.1). Gleichstellungsvorschriften im Personalrecht konkretisieren das Gleichstellungsgesetz, und mit der Durchführung des Lohngleichheitsdialogs hat die BV sich glaubwürdig zum Antidiskriminierungsansatz bekannt. Ohne vom nationalen Wohlfahrtsregime abzuweichen, positioniert sich die BV mit Elementen aus dem Expanding-Opportunities-Ansatz - Förderung der Kinderbetreuung und Laufbahnförderung - als Arbeitgeberin mit guten Sozialleistungen. Als Leitnorm scheint sich die kontinuierlich erwerbstätige Person herauszubilden, die nach der Geburt eines Kindes nur kurz aussetzt und dann allenfalls mit reduziertem Pensum an den Arbeitsplatz zurückkehrt, wobei dies stärker für Frauen als für Männer gilt.

Die heterogene Organisation der betrieblichen Gleichstellungsarbeit ist in der offenen Formulierung rechtlicher Grundlagen angelegt. Für die starke Form im EDA lassen sich politische Gründe ausmachen, die Wahl der beiden anderen Organisationsformen scheint situativ bedingt zu sein. Grundsätzlich bietet eine solche offene rechtliche Formulierung auch Gestaltungsflexibilität für verschiedene Settings und Herausforderungen, so etwa bei den niedrigeren Zielen beim Frauenanteil bei Grenzwache und Verteidigung. Andere 
Ausdifferenzierungen aufgrund sachlicher Voraussetzungen scheinen aber nicht auf.

Die Organisation der Gleichstellungsarbeit zeigt zwischen den hierarchischen Ebenen geteilte Verantwortlichkeiten. Die organisatorische Koordination bei Zielformulierung, Reporting und Controlling funktioniert gut, dabei sind die Gleichstellungsbeauftragten aber nur beratend tätig: Formuliert und entschieden wird auf oberer Leitungsebene. Eine inhaltliche Koordination und Debatte zwischen den verschiedenen Ebenen, Institutionen und AkteurInnen - Amtsleitungen, Personalamt, Personalabteilungen, Beauftragte fehlt aber, und zwar was Ziele, Visionen, gute oder weniger gute Maßnahmen und ihre Umsetzung anbelangt. Eine gemeinsame Gleichstellungspolitik der Bundesverwaltung ist nicht erkennbar. Das Fehlen von qualitativer Koordination und entsprechenden Organisationsentwicklungsressourcen zusammen mit fehlender gesetzlicher Verankerung erklärt auch, warum Gender Mainstreaming eine Absichtserklärung in den Chancengleichheitsweisungen geblieben ist. Die quantitativen Gleichstellungsziele sind gut in die allgemeine Personalpolitik und ihr Controlling integriert. Dort sind sie integraler Bestandteil der Berichte; das Thema wird sodann in „Auskopplungen“, also Extra-Berichten, noch weiter spezifiziert. Weil Gleichstellungspolitik aber wegen der oben genannten Gründe kaum als eigenständiges Handlungsfeld konturiert ist, löst sie sich tendenziell in der Personalpolitik auf und wird auf Eliten sowie quantitative Ziele verengt.

Die hier besprochenen Maßnahmen sind überwiegend Personalentwicklungsmaßnahmen, die auf einzelne Beschäftigte zielen. Sie setzen daher kaum auf der organisatorischen Ebene an, womit ihre Reichweite begrenzt ist. Die Ausnahme sind Workplace Policies im EDA, die auch für die Auslandsvertretungen einen Code of Conduct etablieren.

Auf die geringe rechtliche Verbindlichkeit von Maßnahmen und die Existenz von Gleichstellungsbeauftragten wurde hier immer wieder hingewiesen. Die quantitativen Ziele sind zwar verbindlich sowie terminiert und sie werden regelmäßig kontrolliert. Allerdings gibt es bei Nichterreichen keine Sanktionen. Nimmt man das EPA als Beispiel, so bekommt die Gleichstellungsförderung nur sehr wenig Ressourcen. Allerdings kann die Praxis der Gleichstellungspläne und des Reportings eine „soziale Verbindlichkeit“ aufbauen, pointiert gesagt ein ,naming and shaming“ für hinterherhinkende Einheiten in Gang setzen. Eine solche Verbindlichkeit kann nützlich sein, reicht aber nicht aus.

Die Institutionalisierung wirkt sich auf die umgesetzten Maßnahmen und Erfolge aus. Grundsätzlich zeigt eine stärkere Organisationsform wie beim EDA mit einer zentralen, hoch angesiedelten Stelle und relativ guter Ressourcenausstattung eine höhere betriebliche Sichtbarkeit sowie eine umfassendere Rahmung der Themas, welche über Personalentwicklung hinausgeht und organisationale Aspekte der Chancengleichheit einbezieht. Dabei gibt es 
mehr Maßnahmen, und die Expertise der Gleichstellungsbeauftragten ist wesentlich größer als in anderen Settings. Umfassende langfristige Programme, das zeigt auch das „Package“ des Volkwirtschaftsdepartements, sind erfolgreicher in Bezug auch auf quantitative Ziele. In schwachen institutionellen Settings gibt es weniger Maßnahmen, welche auch weniger eine kulturprägende Wirkung auf die Beschäftigten entfalten, zumal sie argumentativ weniger mit Chancengleichheitszielen verknüpft werden, und zwar unabhängig von ihrer tatsächlichen Relevanz für Gleichstellung. Gleichstellungspolitik in der Bundesverwaltung ist vor allem die Sache von Personalfachleuten und insofern eine Funktion guten Personalmanagements. Einzelne Führungspersonen (wie Departements- oder Amtsleitungen) können zudem die Gleichstellungspolitik stark prägen - negativ wie positiv. Da die bundesrechtlichen Vorgaben für die Verwaltung nur beispielhaft Maßnahmen und Ziele der Politik aufzeigen und weil ein spezifisches Koordinationsgremium fehlt, hängt es relativ stark von Fachpersonen und Vorgesetzten ab, welche Maßnahmen eingesetzt werden, was die Heterogenität fördert. Dies erklärt auch die Verengung auf eine Gleichstellungspolitik als Funktion guten Personalmanagements.

Die eingesetzten Maßnahmen und Instrumente in der betrieblichen Gleichstellungspolitik sind grundsätzlich konsistent. Bis auf die Subventionspolitik bei der Kinderbetreuung konnten keine widersprüchlichen Anreize ausgemacht werden. Dessen Effekt auf die vertragliche Arbeitszeit dürfte auch relativ gering sein. Unterstützung bei der Kinderbetreuung, Teilzeitmöglichkeiten bei Bevorzugung großer Teilzeit und Instrumente zur Laufbahnförderung zielen auf die kontinuierlich erwerbstätigen Eltern, die neben ihrem anspruchsvollen Job auch noch Verantwortung in der Familie wahrnehmen können sollen. Dabei entwickeln Programme mit mehreren gleichzeitig umgesetzten bzw. angebotenen Maßnahmen größere Schubkraft. Ebenfalls Schub für erfolgreiche betriebliche Gleichstellungspolitik bringen vor allem das Engagement und die Initiativen von BundesrätInnen (dabei wurden Ruth Dreifuss, Michéline Calmy Rey und Doris Leuthard mehrfach erwähnt) sowie das Engagement der oberen Führungsebene. Ein nachlassendes Commitment auf dieser Ebene wird von den Beschäftigten und ExpertInnen sofort realisiert.

Betriebliche Gleichstellung ist ein Querschnittsthema. Sie braucht eine starke fachliche Koordination auf der Grundlage eines gemeinsamen Problem- und Zielverständnisses sowie auf der Grundlage einer Reflexion über wesentliche Gemeinsamkeiten und Besonderheiten in den einzelnen Einheiten der Bundesverwaltung. Eine solche eigenständige Koordinierung hätte sehr wahrscheinlich mit institutionellen Widerständen zu rechnen. Dennoch könnte das Handlungsfeld schärfer konturiert werden, und man könnte dort auch neue Fragen bearbeiten: So wurde das Controlling 2013 stark vereinfacht. Weil aber das, was nicht erhoben und nicht gesagt wird, auch nicht als 
Problem identifiziert werden kann, sollte eine erweiterte Datenerhebung kritische Punkte prüfen - beispielhaft sei hier die Frage genannt, ob die Vereinbarkeitspolitik tatsächlich wirkt und weniger junge Mütter aus der Bundesverwaltung aussteigen. 



\section{Berufliche Gleichstellung im deutschen Bundesdienst: Vorbild für die widerständige Privatwirtschaft?}

\section{Sophie Rouault}

Über die Erfolge, die Deutschlands Gleichstellungspolitik in den vergangenen 30 Jahren erzielte, gibt es in der Literatur ausdifferenzierte Ansichten. Manche sehen diesen Politikbereich als ,eine der erfolgreichsten politischen Innovationen“ (Holland-Cunz 2006; siehe auch Rudolph 2009), vor allem angesichts der steigenden Anzahl von Gleichstellungsbeauftragten auf allen Ebenen der Verwaltung. Andere beklagen den „Ritt auf der Schnecke“, mit dem die überaus zähe Dynamik einer deutschen Gleichstellungspolitik (Berghahn 2011) beschrieben wird, die oft nur unter richterlichem oder politischem Druck (vor allem der Europäischen Union) fortschreitet (Kodré/Müller 2003).

Wenn aber die gesetzliche Regulierung der beruflichen Geschlechtergleichstellung bei privaten und öffentlichen Arbeitgebern verglichen wird, erscheint ein kontrastreiches Bild, das diese widersprüchlichen Analysen erklärt. Der Kontrast zwischen einer nur minimal regulierten Privatwirtschaft und einem stark regulierten öffentlichen Dienst (auf alle Regierungsebenen) ist doch frappierend. Während sich (private) Arbeitgeberverbände mit dem Argument der Autonomie des unternehmerischen Handelns gegen die gleichstellungspolitische Einmischung des Staates erfolgreich zu wehren wussten wie das Scheitern eines breit angelegten Gleichstellungsgesetzes 2001 es zeigte -, wurde der öffentliche Dienst Gegenstand eines regelmäßig novellierten Gesetzes. Dem Bundesdienst wird im politischen Diskurs öfter eine kompensatorische Rolle für die Unterregulierung der Privatwirtschaft zugeschrieben: Die Bundesverwaltung soll für private Unternehmen in gleichstellungspolitischen Fragen ein Vorbild sein (Rudolph/Schirmer 2004: 70).

Im Anschluss an die Fragestellungen des Ländervergleichs zwischen der Schweiz, Deutschland und Österreich wird zunächst das in Deutschland gespaltene Regime zur beruflichen Gleichstellung in seinen historischen und institutionellen Grundauszügen auf Basis einer Literaturanalyse skizziert und durch ein statistisches Bild der neueren Entwicklungen der weiblichen Beschäftigung im deutschen Bundesdienst zusammengefasst, das über die vergleichenden Daten im dritten Kapitel in diesem Buch hinausgeht. Im Anschluss daran wird die Ausgestaltung der Instrumente der Gleichstellungspolitik für den Bundesdienst untersucht, und die Voraussetzungen ihrer Wirksamkeit werden bewertet. Abschließend werden die (normative) Kohärenz und die (technische) Konsistenz des deutschen Policy-Regimes zur berufli- 
chen Gleichstellung im Bundesdienst bewertet und damit dem Vorbildanspruch der Bundesverwaltung entsprechend spezifiziert.

\subsection{Kontextbedingungen eines gespaltenen Regimes zur beruflichen Gleichstellung}

In dem Versuch, die konservative Tradition des männlichen Ernährers in manchen Politikbereichen (Steuerpolitik, Familienpolitik) zu erhalten und in anderen zu modernisieren (Bildungspolitik, Vereinbarkeitspolitik), weist das deutsche Genderregime widersprüchliche Züge auf. Es muss daher als ein hybrides Regime bezeichnet werden (Bothfeld 2008), das nur langsam und begrenzt die Züge eines Doppelverdienermodells annimmt und deswegen eher als ein „Anderthalb-Verdiener-Modell“ (Bothfeld et al. 2010: 35) verstanden werden kann.

Im engeren Bereich der beruflichen Gleichstellung findet man dieses gemischte Bild wieder. Deutschland erreichte schon 2006 eine Beschäftigungsquote von $60 \%$ bei Frauen im Alter von 19 bis 64 Jahren und erfüllte damit eines der im Jahr 2000 auf europäischer Ebene vereinbarten „Lissabon-Ziele“ für eine wettbewerbsfähige, nachhaltige und wissensorientierte Wirtschaft. Die Beschäftigungsquote nähert sich nun dem neuen Ziel von $75 \%$ der neuen Europa-2020-Strategie an - bereits 2013 lag die Beschäftigungsquote von Frauen bei $72,3 \%$. Aufgrund des steigenden Anteils an geringfügig und teilzeitig beschäftigten Frauen lag diese Quote - berechnet in Vollzeitäquivalenten -2012 jedoch nur bei 55,2\% (45\% im Jahr 2005). ${ }^{1}$ Dies zieht sowohl ein Armutsrisiko als auch ein Altersarmutsrisiko für Frauen wegen mangelnden existenzsichernden Einkommens bzw. zu geringer Alterssicherung nach sich (Pimminger 2012a, b). Dieser geringe Beschäftigungsgrad lässt sich hauptsächlich durch den fortgesetzten Mangel an Betreuungsplätzen für Kinder unter drei Jahren erklären (Bothfeld et al. 2010: 43). Im März 2013 lag die Betreuungsquote der Kinder unter drei Jahren immer noch bei 29,3\% (das Lissabon-Ziel für 2010 betrug 33\%). Noch ist unklar, inwiefern die Einführung des gesetzlichen Anspruchs auf einen Betreuungsplatz für alle UnterDrei-Jährigen im August 2013 dazu beiträgt, dieses Angebotsdefizit zu überwinden. Zudem erklärt sich die Persistenz der Teilzeitarbeit bei Frauen durch die Halbtagsangebote der Schulen und Kindertagesstätten sowie die innerfamiliäre Arbeitsteilung (Pimminger 2012b). Bei der sektoralen und hierarchischen Segregation am Arbeitsmarkt verläuft die Entwicklung noch langsamer oder ist gar rückläufig. Zwischen 1997 und 2007 ist die berufliche Segrega-

1 Alle hier zitierte Statistiken beziehen sich, wenn nicht anders angegeben, auf die folgende Quelle: Statistisches Bundesamt. 
tion am deutschen Arbeitsmarkt - gemessen am IP-Index ${ }^{2}$ - leicht (von 17,8 auf $18,4 \%$ ) gestiegen. Bei der hierarchischen Segregation verändert sich das Bild nur unter hohen politischen Druck, der durch die Debatte um die Einführung einer gesetzlichen Geschlechterquote für Aufsichtsräte um 2010 in Deutschland entstand. In den 100 größten deutschen Unternehmen betrug 2006 der Frauenanteil in den Aufsichtsräten 9,6\%, um 2010 auf 10,6\% und dann allmählich bis 2014 auf 18\% anzusteigen. In den Vorständen stieg der Frauenanteil hingegen viel moderater: ausgehend von $0,2 \%$ in 2006 erreichte dieser mühsam 5,4\% in 2014 (Holst/Kirsch 2015).

Obwohl die Bundesregierung durch die 1994 eingeführte Ergänzung des Art. 3 Abs. 2 des Grundgesetzes ${ }^{3}$ sich zum aktiven Handeln bei der Frauenförderung verpflichtet hat, spiegeln diese Zahlen die Tatsache wider, dass es in Deutschland bis heute keine explizite politische Strategie zur Förderung der beruflichen Gleichstellung gibt, die sich auf den gesamten Arbeitsmarkt beziehen würde (Wahl 1999; Bothfeld et al. 2010). Eine Reihe von Einzelinitiativen, die auf die Gleichstellung in den Landesdiensten und im Bundesdienst zielen, ist wohl aber zu beobachten.

\subsubsection{Berufliche Gleichstellung in der Privatwirtschaft: Die betriebliche Freiheit, zu handeln}

Das frauenpolitische Programm der SPD „Frau und Beruf“ von 1998 war der erste und bisher einzige umfassende Programm, das auf eine breit angelegte Förderung der Gleichstellung von Frauen am Arbeitsmarkt zielte (Bothfeld 2005; Geppert 2009). Dies sah u.a. ein Gesetz für die Privatwirtschaft vor, das sich nach zähen Kämpfen aber als nicht durchsetzbar erwies (Koch 2003). Anstelle des Gesetzes schlossen Regierung und Arbeitgeberverbände 2001 eine ,freiwillige Vereinbarung“, in der die Arbeitgeber sich verpflichteten, Maßnahmen zur betrieblichen Förderung der Frauen zu ergreifen. Die zweijährlichen Bilanzen der Umsetzung der Vereinbarung deuten aber auf andauernde Defizite hin, besonders in den Punkten Entgeltgleichheit ${ }^{4}$ und Förderung von Frauen in Führungsposition. Seit der Reform des Betriebsver-

2 Der (sektorale) IP-Index (,sectoral IP index“) bezeichnet den Anteil der Beschäftigten, der die Beschäftigung wechseln sollte, um eine geschlechtergerechte Verteilung quer aller beruflichen Sektoren zu erzielen (0 kennzeichnet die perfekte Gleichstellung und 50 den höchsten Segregationsgrad). Der IP-Index wird als Referenz-Indikator in der Europäischen Beschäftigungsstrategie angewandt (Bettio/Verashchagina 2009: 31).

3 Der Artikel 3 (,Männer und Frauen sind gleichberechtigt“) von 1949 wurde mit dem folgenden Absatz ergänzt: „Der Staat fördert die tatsächliche Durchsetzung der Gleichberechtigung von Frauen und Männern und wirkt auf die Beseitigung bestehender Nachteile hin.“

4 In europäische Rankings bezieht Deutschland mit Regelmäßigkeit eine der letzten Positionen unter den 27 EU-Mitgliedsländer: Der nicht standardisierte Gender-Pay-Gap betrug 2012 22\%, und Deutschland war damit an vorletzten Position (European Commission 2014: 21). 
fassungsgesetzes (BVG) im Jahr 2001 ist die Förderung der Geschlechtergleichstellung auch eine Aufgabe des Betriebsrats, der jährlich in der Betriebsversammlung darüber zu berichten hat (Klenner 2004). Allerdings zeigte eine Bestandsaufnahme, dass dies 2003 nur in $11 \%$ der Betriebe umgesetzt wurde (ebd.: 280) und nur in 3\% den Betriebe eine Betriebsvereinbarung zur Chancengleichheit galt (ebd.: 278).

Erst im Jahr 2006 wurde im Zuge der europäischen Rechtsangleichung das Allgemeine Gleichbehandlungsgesetz (AGG) verabschiedet, das erstmals eine solide Basis für Klagen gegen direkte und indirekte Diskriminierung (nicht nur aufgrund des Geschlechts) schuf. Wenn auch das AGG die Beweislast auf den Arbeitgeber verlagert, ein ,angemessenes“ Entschädigungsniveau vorschreibt und eine Antidiskriminierungsstelle institutionalisiert, so fehlen doch die Möglichkeiten zur Kollektiv- oder Stellvertreterklage sowie eine Regelung zu einer gleichstellungsgerechten Vergabepraxis bei öffentlichen Aufträgen (Bothfeld et al. 2010: 31). In diesem spärlichen gesetzlichen Kontext genießen deutsche Arbeitgeber in der Privatwirtschaft eine große Freiheit zur Umsetzung beruflicher Gleichstellung. So hängt die gleichstellungspolitische Praxis der Betriebe vom alltäglichen Engagement der Betriebsräte und der Geschäftsführung ab.

Die seit Mitte der 1990er Jahren von der Bundesregierung entwickelten und den Betrieben zu Verfügung gestellten Instrumente setzen vor allem auf die freiwillige Selbstkontrolle der Betriebe in Sachen Gleichstellung, so etwa die nach begleiteter Selbstevaluierung gewährten Prädikate „total equality“ (1996) oder „familienfreundlicher Betrieb“ (1998), den Leitfaden zur Entgeltgleichheit (2003) oder das Instrument „Logib-D“ (2009) zur Prüfung der tariflichen und betrieblichen Lohnstrukturen. Da diese nicht bindenden Regelungen und Instrumente zu wenig substanziellen Fortschritten geführt haben, hat die Große Koalition, die Ende 2013 die Regierungsgeschäfte übernahm, einen neuen gleichstellungspolitischen Kurs eingeleitet: Im Koalitionsvertrag wurden eine gesetzliche Geschlechterquote für Aufsichtsräte und eine Berichtspflicht zur Entgeltgleichheit angekündigt. Das im März 2015 angenommene Gesetz zur Förderung der Frauen in Führungspositionen setzt nun eine Mindestquote von 30\% (ab 2016) in Aufsichtsräten von börsennotierten und voll mitbestimmungspflichtigen Unternehmen durch. ${ }^{5}$ Diese Quote wird von einer Verpflichtung zur Festlegung von Zielgrößen für Aufsichtsräte, Vorstände und die oberste Managementebene in mitbestimmten oder börsennotierten Unternehmen flankiert. Die Sanktion bei Nichterfüllung des Quotenwerts ist jedoch relativ mild, und eine Berichtspflicht zur beruflichen Gleichstellung für Privatarbeitgeber wird in nur sehr begrenzter Form abverlangt (Bothfeld/Rouault 2015).

5 Knapp 100 Betriebe sind von der festen Quotenvorgabe für Aufsichtsräte betroffen, aber 3.500 zur Festlegung von Zielgrößen verpflichtet (Pütz/Weckes 2014). 


\subsubsection{Berufliche Gleichstellung im Bundesdienst: Inkrementelle Institutionalisierung eines Politikfelds}

Grundlegend für die berufliche Gleichstellung von Frauen und Männern im Bundesdienst ist Art. 33 des Grundgesetzes, der vorschreibt, dass jeder Deutsche „nach seiner Eignung, Befähigung und fachlichen Leistung gleichen Zugang zu jedem öffentlichen Amt" hat. Dieses Grundrecht fand aber erst 1953 seine Konkretisierung, als das neue Bundesbeamtengesetz die sog. „Zölibatsklausel“ aufhob, die verheiratete Frauen daran hinderte, nach ihrer Heirat als Beamtinnen weiter beschäftigt zu bleiben (Mecking 2005). ${ }^{6}$ Damit wurde die auffälligste (direkte) Diskriminierung von Frauen im öffentlichen Dienst beendet und damit auch das allgemeine verfassungsrechtliche Gleichheitsgebot formal erfüllt. Mit der Aufhebung des Teilzeitverbots im Bundesdienst für Beamtinnen (1969) und Beamte (1971) mit Kindern wurde eine „praktische Gleichberechtigung“ angestrebt und der quantitative Anstieg des Frauenanteils im öffentlichen Dienst ermöglicht (von Oertzen 1999b). Diese gesetzlichen Meilensteine stellen die ersten rechtlichen Schritte eines Wandels des deutschen (föderalen) Staates als Arbeitgeber dar, der sich von dem Abbild einer institutionalisierten Diskriminierung von Frauen distanzierte und begann, eine Vorbildrolle zu beanspruchen.

Von einer regelrechten gleichstellungspolitischen Strategie für den Bundesdienst kann aber erst ab Ende der 1980er Jahren gesprochen werden. Zuvor sind lediglich einzelne themen- und maßnahmenbezogene Initiativen innerhalb der Fachressorts zu erkennen, u.a. die Einrichtung von „Frauenreferaten“ in einigen Bundesministerien (Geppert/Lewalter 2012). Ein „Arbeitsstab Frauenpolitik" versuchte durch Forschungs- und Modellvorhaben sowie Öffentlichkeitsarbeit ressortübergreifend gleichstellungssensibilisierend $\mathrm{zu}$ agieren. Die Gründung einer Abteilung für Frauenpolitik und die Übertragung der Federführung und der Gesetzgebungskompetenz für Frauenpolitik an das Bundesministerium für Jugend, Frauen und Gesundheit kennzeichnete 1987 den Institutionalisierungsbeginn eines neuen Politikfeldes (ebd.). Eine kurze Richtlinie wurde 1986 erstellt, die 1994 in einem ersten eigenen Gesetzespaket ausdifferenziert und formalisiert wurde: So enthält das Gleichstellungsdurchsetzungsgesetz (DGleiG), auch ,2. Gleichberechtigungsgesetz“); das sogenannte „Frauenförderungsgesetz" (FFG) und das Bundesgremienbesetzungsgesetz (BGremBG). Von den gleichstellungspolitischen Regelungen auf kommunaler und Länderebene inspiriert, wurden damit die wichtigsten Institutionen der deutschen Gleichstellungspolitik im Bundesdienst geschaffen - die ministeriellen Gleichstellungsbeauftragten und Gleichstellungspläne der einzelnen Ressorts und die Berichtspflicht der Bundesregierung. Damit

6 Zeitweilig beseitigt in der Weimarer Republik, wieder eingeführt unter der nationalsozialistischen Regierung, wurde die „Zölibatsklausel“ 1950 in eine „Kann-Bestimmung“ umgewandelt, bevor sie 1953 aufgehoben wurde (Mecking 2005: 478-483). 
wurden die grundlegenden Akzente gesetzt in Richtung einer gleichstellungsorientierten Personalpolitik der einzelnen Ressorts einerseits und einer stark auf Vereinbarkeit orientierten Gleichstellungspolitik anderseits.

Die 1999 durchgeführte Verankerung von Gleichstellung als Leitprinzip für alle ,politischen, normgebenden und verwaltenden Maßnahmen der Bundesministerien“" in der gemeinsamen Geschäftsordnung der Bundesministerien (GGO) - auch als ,gender mainstreaming"-Strategie bezeichnet (Schweikert 2002), kennzeichnet den Anfang einer zweiten Institutionalisierungswelle der Gleichstellungspolitik in der Bundesverwaltung unter der RotGrünen Koalition. Dem Scheitern des vorgesehenen Gleichstellungsgesetzes für die Privatwirtschaft folgte das Bundesgleichstellungsgesetz (BGleiG) 2001, das das im FFG verfügbare Instrumentarium noch ausdifferenzierte: Es stärkte die Funktion der Gleichstellungsbeauftragten, führte eine Quotenregelung bei Einstellung und Aufstieg ein und erweiterte den Anwendungsbereich des Gesetzes, sodass dieses Regelwerk nun als Vorbild für Ländergesetze gilt (Rudolph/Schirmer 2004: 73).

Tafel 5.1: Gesetzliche Grundlagen der Gleichstellungspolitik im Bundesdienst

\footnotetext{
- Richtlinie zur beruflichen Förderung von Frauen in der Bundesverwaltung (1986).

- DGleiG (1994), „2. Gleichberechtigungsgesetz“: Gesetz zur Durchsetzung der Gleichberechtigung von Frauen und Männern; ein Gesetzespaket, das zwei Gesetze enthält:

- FFG (1994), sog. „Frauenförderungsgesetz“ (Art. 1 DGleiG): Gesetz zur Förderung von Frauen und Männern und der Vereinbarkeit von Familie und Beruf in der Bundesverwaltung und den Gerichten des Bundes;

- BGremBG (1994), sog. „Bundesgremienbesetzungsgesetz“ (Art. 11 DGleiG): Gesetz über die Berufung und Entsendung von Frauen und Männer in die Gremien im Einflussbereich des Bundes.

- BGleiG (2001) (sog. „Bundesgleichstellungsgesetz“): Gesetz zur Gleichstellung von Männern und Frauen in der Verwaltung und den Gerichten des Bundes.

- AGG (2006) (sog. „Allgemeine Gleichbehandlungsgesetz“): Umsetzung europäischer Richtlinien zur Verwirklichung des Grundsatzes der Gleichbehandlung.

- Gesetz für die gleichberechtigte Teilhabe von Frauen und Männern an Führungspositionen in der Privatwirtschaft und im öffentlichen Dienst (2015) - Novellierung des BGleiG und des BGreG.
}

Quelle: eigene Zusammenstellung

Das Allgemeine Gleichbehandlungsgesetz (AGG) von 2006 führte neue Begriffsbestimmungen der unmittelbaren und mittelbaren Benachteiligung (u.a. wegen des Geschlechts) und der sexuellen Belästigung ein. Es setzt eine lange erwartete Anpassung an die europäische Gesetzgebung um und ergänzt die institutionelle Strategie der Frauenförderung durch einen aktivierenden Antidiskriminierungsansatz (von Wahl 1999).

Der Vernachlässigung der Gender-Mainstreaming-Strategie und die Schließung des für die Begleitforschung gegründeten „Genderkompetenzzentrums“" an der Humboldt-Universität zu Berlin im Jahr 2009 bildeten die 
logischen Folgen einer Neuorientierung der Gleichstellungspolitik unter der zweiten Rot-Grünen Regierung (Lewalter et al. 2009). Diese politische Umkehr unterstreicht die prekäre Institutionalisierung einer Politik, deren Instrumente bei der Implementation immer erneut verhandelt werden müssen (Rudolph/Schirmer 2004: 227).

Während das 2015 angenommene Gesetz für die gleichberechtigte Teilhabe von Frauen und Männern an Führungspositionen in der Privatwirtschaft und im öffentlichen Dienst durch die Einführung einer verbindlichen Quote für die Privatwirtschaft (siehe oben) ein starkes politisches Signal darstellt, bringt die begleitende Novellierung des BGleiG und des BGremG kein wesentliches Novum: Die Förderung von Frauen in Führung wird durch keinen neuen Ansatz unterstützt, und die geplante ,alternierende Männerförderung “7 lässt sogar eine deutliche Aufweichung des Bekenntnisses zu einer substanziellen Gleichstellungspolitik im Bundesdienst befürchten (Deutscher Juristinnenbund 2014).

Diese kurze Chronologie der Entwicklung der Bundesgleichstellungspolitik deutet tatsächlich auf eine inkrementelle, aber auch fragile Institutionalisierung hin. Diese Dynamik wird in der Literatur kritisch bewertet: Für Angelika von Wahl hat Deutschland den „Weg einer Überinstitutionalisierung und einer weitgehend symbolischen Politik eingeschlagen" (von Wahl 1999: 124). Dieser ,prozedurale Weg“, von allgemeinen Geboten ohne Sanktionen gekennzeichnet, kann hauptsächlich „bereichsspezifische, begrenzte und individuelle Erfolge" erzielen und kaum strukturelle Veränderungen bewirken (Rudolph/Schirmer 2004: 16; siehe auch Rudolph 2009). Die Institutionalisierung kann sogar Rückschritte erleiden (Lang 2009).

\subsubsection{Gespaltenes Policy-Regime zur beruflichen Gleichstellung}

Das Profil des deutschen Policy-Regimes zur beruflichen Gleichstellung erscheint zwischen den Steuerungsmodi, die einerseits die Privatwirtschaft und anderseits den öffentlichen Dienst regulieren, gespalten. Die Steuerung der beruflichen Gleichstellung im öffentlichen Dienst wird durch eine inkrementelle Dynamik gekennzeichnet, die aber unter einem andauernden Defizit an ressortübergreifender Koordinierung und an diskontinuierlicher politischer Führung leidet. Dieses Defizit ist vor allem durch die streng bewahrte Autonomie der einzelnen Verwaltungseinheiten (Ministerien) zu erklären. Die Steuerung der beruflichen Gleichstellung in der Privatwirtschaft ist eher durch eine „renitente“ Dynamik kennzeichnet, die trotz wiederholter Versuche staatlicher Regulierung bislang hauptsächlich auf die Freiwilligkeit der Arbeitgeber setzt. Programme zur Förderung der Selbstregulierung werden

7 Das heißt, je nachdem, welches Geschlecht in einem der Bereiche unterrepräsentiert ist, kann das Bevorzugungsprinzip nun für Männer angewandt werden. 
von der Regierung zwar zu Verfügung gestellt, aber kaum von hierarchischen oder institutionellen Maßnahmen begleitet, die ihre Chancen auf Umsetzung erhöhen würden.

Im Folgenden werden die gleichstellungspolitischen Initiativen im öffentlichen Dienst - genauer in den obersten Bundesbehörden, d.h. in den Bundesministerien - in den Blick genommen. Am Beispiel der einzelnen Instrumente zur Herstellung beruflicher Gleichstellung im Bundesdienst wird gezeigt, wie deren Design, das sich sowohl aus den gesetzlichen Vorgaben, aber auch aus der Praxis der einzelnen Verwaltungseinheiten ergibt, ihre Wirksamkeit bestimmt. Unzureichende Verbindlichkeit, Ausdifferenziertheit oder Reichweite verstärken die technische Inkonsistenz dieses Policy-Regimes, wobei einzelne personalpolitische Strategien in den Bundesministerien diese strukturelle Schwäche zuweilen ausgleichen können.

\subsection{Neuere Entwicklungen beim deutschen Bundespersonal: Immer mehr Frauen (in Teilzeit)}

2011 entfielen auf den öffentlichen Dienst 11,5\% der deutschen Gesamtbeschäftigung, womit Deutschland deutlich unter dem Durchschnitt der OECDLänder lag (15,1\% im Jahr 2011). ${ }^{8}$ Im Juni 2013 waren im deutschen öffentlichen Dienst 4,635 Mio. Personen beschäftigt, darunter 55\% Frauen. 2,35 Mio. Personen (50,7\%) waren im Landesbereich, 1,4 Mio. (30,3\%) im kommunalen und $503.900(10,8 \%)$ im Bundesbereich, weitere $8 \%$ bei den Trägern der Sozialversicherung beschäftigt. Von den rund 4,6 Mio. Beschäftigten waren wiederum 36\% BeamtInnen und RichterInnen, 59\% Angestellte und 3,7\% SoldatInnen. Der Frauenanteil betrug 60\% im kommunalen Bereich, $52 \%$ im Landesbereich, aber nur rund $26 \%$ im Bundesbereich. ${ }^{9}$

8 „Total employment in the general government sector as a percentage of labour force (2011)“, Data from International Labour Organization (ILO) LABORSTA Database and OECD National Accounts Statistics: http://www.oecd.org/gov/pem/OECD\%20HRM\%20 Profile\%20-\%20Germany.pdf (Zugriff: 24.10.2015).

9 Diese Statistiken stammen aus der allgemein öffentlich zugänglichen Statistik zum Personal des öffentlichen Dienstes (Statistisches Bundesamt, Fachserie 14, Reihe 6). Der Bundesbereich wird wie folgt definiert: Kernhaushalt und Sonderrechnungen (einschließlich Bundeseisenbahnvermögen) des Bundes sowie Einrichtungen in öffentlich-rechtlicher Rechtsform, die unter der Rechtsaufsicht des Bundes stehen, ohne Sozialversicherungsträger und Bundesagentur für Arbeit (öffentlicher Dienst im Bundesbereich). Hinzu kommen die Einrichtungen in privater Rechtsform, die sich mehrheitlich im Besitz des Bundes befinden (öffentliche Arbeitgeber im Bundesbereich). Der Kernhaushalt wiederum enthält alle Ämter, Behörden, Gerichte und Einrichtungen, für die in den Haushaltsplänen des Bundes die Ausgaben und Einnahmen brutto veranschlagt und Personalausgaben ausgewiesen werden. Die Ministerien gehören zum Kernhaushalt bilden nur einen (kleineren) Teil des Bundesbereichs. 
Die obersten Bundesbehörden (zu denen auch die Bundesministerien zählen, auf die die folgende Analyse sich bezieht) sind jedoch keinesfalls eine Männerdomäne: Der Frauenanteil hat 2012 immerhin mit 51,2\% gut die Hälfte der Beschäftigten erreicht (siehe Abb. 5.1), wobei der Frauenanteil bei den Angestellten $(66,6 \%)$ wesentlich höher liegt als bei den BeamtInnen $(40,2 \%) .{ }^{10}$

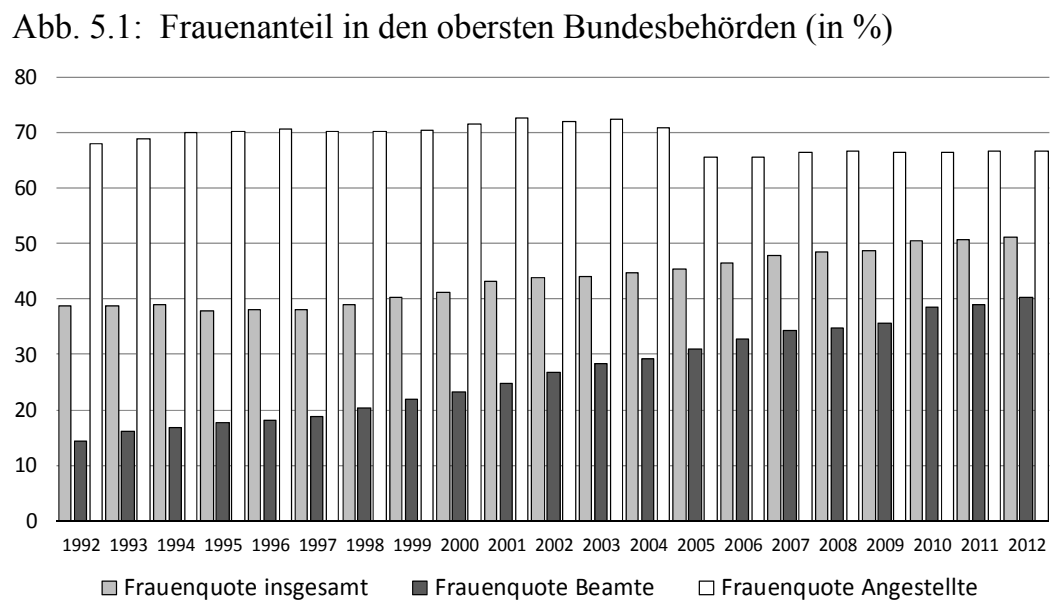

Quelle: Frauenförderstatistik (1992-2002); Gleichstellungsstatistik (nicht öffentl., vgl. Fn. 10 in diesem Kapitel) ab 2003

Der Beschäftigungszuwachs der Frauen in der gesamten Bundesverwaltung hat überwiegend im Bereich der Teilzeitarbeit stattgefunden: Während die Anzahl der weiblichen Beschäftigten zwischen 2004 und 2009 um 17.375 wuchs, stieg die Zahl der teilzeitbeschäftigten Frauen um 12.424 und die Zahl der vollzeitbeschäftigten Frauen um 4.951 (Deutscher Bundestag 2010a: 15f.).

In den obersten Bundesbehörden sind die Teilzeitbeschäftigten in überwiegendem Maße weiblich. Im früheren Bundesgebiet lag die Teilzeitquote der Frauen noch bei 20\% (1\% bei den Männern). Nach der Wiedervereinigung verringerte sich dieser Anteil zunächst auf 17\% (1991), stieg aber 1994 mit der Einführung der Altersteilzeit auf ein bis dato unerreichtes Niveau von $25 \%$ an (3\% bei Männern). Mit der Ausgliederung der Altersteilzeit aus der

10 Diese Zahlen stammen aus der sog. Gleichstellungsstatistik der Bundesregierung, die nicht öffentlich zugänglich ist. Auskünfte werden jedoch auf gezielte und juristisch geprüfte Anfrage erteilt. Die Erfahrungsberichte der Regierung stützen sich zum größten Teil auf diese Datenquelle, die aber wiederum mit der allgemeinen Personalstatistik des statistischen Bundesamtes aufgrund unterschiedlicher Definitionen nicht harmonisiert ist. So wies die Personalstatistik 2013 z.B. einen Frauenanteil von ,nur“ 48,9\% in der ,politischen Führung" des Bundesdienstes (d.h. in den Ministerien) aus. 
Statistik (1995) stagnierte die Teilzeitquote der Frauen bis zur Einführung des Bundesgleichstellungsgesetzes (2001), anschließend stieg sie noch einmal leicht an. Seit 2005 ist eher eine Stagnation der Teilzeitquote bei Frauen zu beobachten (Abb. 5.2). Bei der männlichen Teilzeitquote war ein starker Anstieg unter der ersten rot-grünen Regierung zu verzeichnen, der aber nicht anhält.

Abb. 5.2: Teilzeitquote in den obersten Bundesbehörden (1992-2012, in \%)

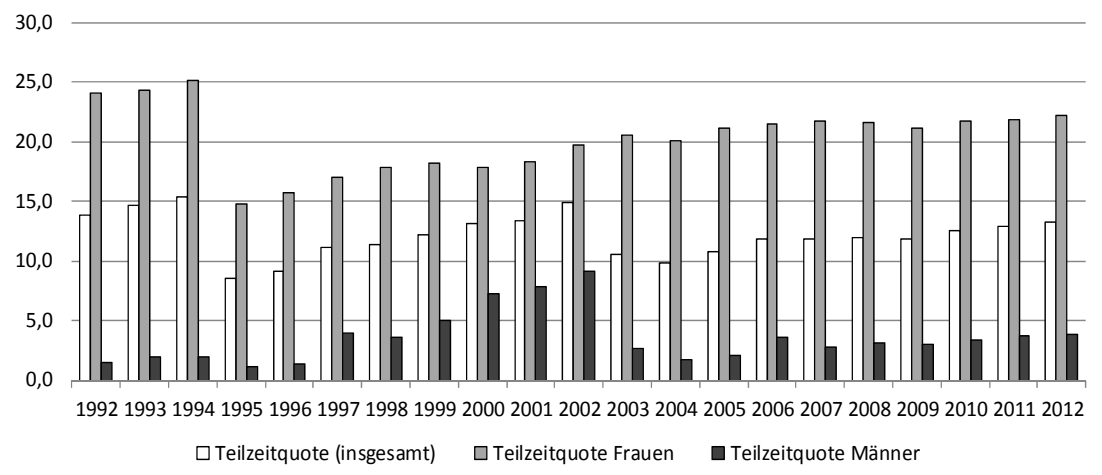

Quelle: Frauenförderstatistik (1992-2002); Gleichstellungsstatistik (nicht öffentlich, vgl. Fn. 10 in diesem Kapitel) ab 2003

Im höheren Dienst zählen Abteilungsleitungen, Unterabteilungsleitungen sowie Referatsleitungen zu den Führungspositionen. ${ }^{11}$ Der Anteil der Männer in einer Führungsposition in den obersten Bundesbehörden war 2009 mit $14,7 \%$ etwa dreimal so hoch wie der der Frauen (4,7\%; Deutscher Bundestag 2010a: 23).

Wenngleich deutliche Fortschritte zu verzeichnen sind, waren 2012 nur etwa $17 \%$ bzw. $18 \%$ der Abteilungs- sowie der Unterabteilungsleitungen weiblich; gerade im Bereich der Abteilungsleitungen, die als „,politische Posten" betrachtet werden, wurde mit dem Wechsel zur rot-grünen Koalition ein großer Zuwachs von 2\% (1998) auf fast 22\% (2004) erreicht. Interessant ist jedoch, dass sich diese Fortschritte kaum fortsetzen.

Bei den Referatsleitungen lässt sich hingegen ein seit Mitte der 1990er Jahre andauernder Anstieg des Frauenanteils beobachten: War 1997 nur jede zehnte Referatsleitung mit einer Frau besetzt, belief sich der Anteil 2009 schon auf rund $26 \%$ und 2012 sogar auf 30\%. Der gleiche Trend gilt insgesamt für den höheren Dienst in den Bundesbehörden, wo sich der Frauenanteil sukzessive von $18 \%$ auf $35 \%$ und $40 \%$ erhöhte (Abb. 5.3). Diese paralle-

11 Während für die Referatsleitungen und Unterabteilungsleitungen Laufbahnbeurteilungen eine zentrale Rolle spielen, werden die Abteilungsleitungen durch die Abwägung politischer Faktoren besetzt. 
len Entwicklungen sind wichtig, da hiermit ein Potenzial von Frauen ,heranwächst', die sich für die nächste Stufe an Führungspositionen überhaupt qualifizieren kann.

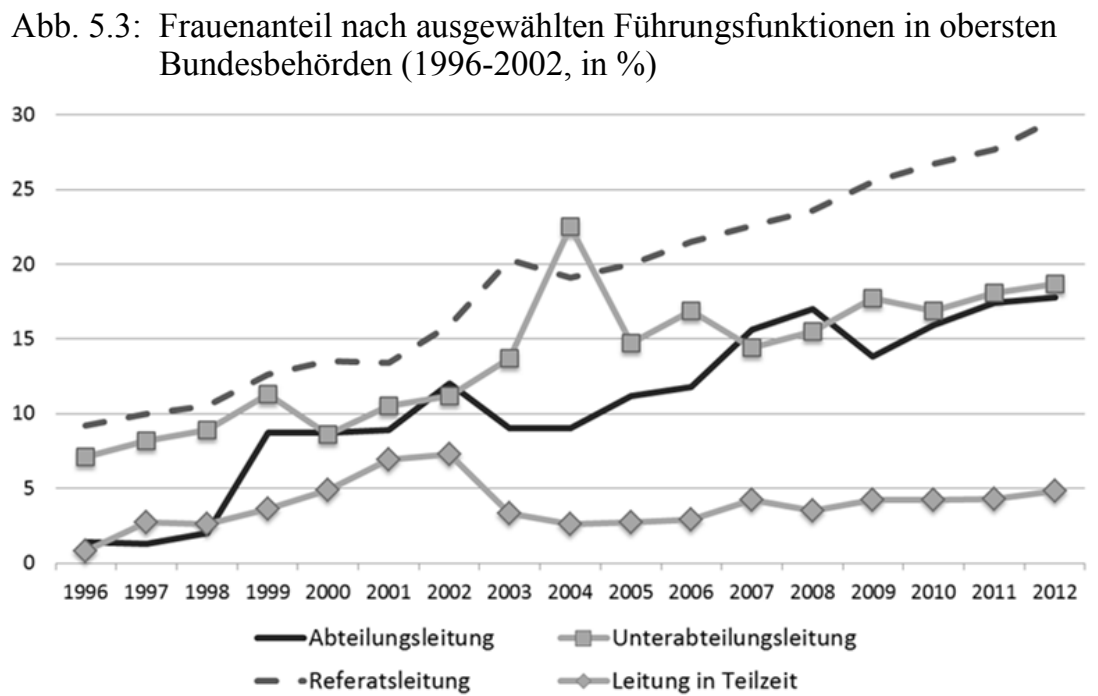

Quelle: Frauenförderstatistik (1996-2002), Gleichstellungsstatistik (nicht öffentlich, vgl. Fn. 10 in diesem Kapitel) ab 2003

In der 2012 vom Beratungsunternehmen Kienbaum vorgelegten statistischen Analyse wird ein Vergleich der Frauenanteile von Führungskräften in den Bundesministerien präsentiert (Kienbaum 2012: 14). Dabei schneidet das BMFSFJ mit 50\% mit am besten ab, während das Auswärtige Amt und das Verteidigungsministerium die Schlusspositionen besetzen (mit 17\% und 8\%). Die in unserer Studie untersuchten Bundesministerien - das Arbeitsministerium und das Finanzministerium - schneiden jeweils leicht über- und deutlich unterdurchschnittlich ab $(30 \%$ und $17 \%$ bei einem Gesamtdurchschnitt von $29,1 \%)$.

\subsection{Institutionelle Gleichstellungspolitik in der deutschen Bundesverwaltung: Eine inkrementelle Dynamik}

Zur Institutionalisierung einer Gleichstellungspolitik in der Bundesverwaltung tragen drei Instrumente wesentlich bei: der Gleichstellungsbericht der Bundesregierung an das Parlament und - in den einzelnen Bundesministerien - das Amt der Gleichstellungsbeauftragten sowie der Gleichstellungsplan der 
Dienststelle. Das Bundesfamilienministerium (BMFSFJ) hat die Federführung und die Gesetzgebungskompetenz in diesem Politikfeld und ist für die Berichtspflicht der Regierung an das Parlament über die erfolgten Fortschritte der Bundesverwaltung beim Abbau geschlechterspezifischer Benachteiligung und Diskriminierung verantwortlich. Die Gleichstellungsbeauftragten (GBAs) begleiten und kontrollieren die Umsetzung der Gleichstellungsgesetze in den einzelnen Bundesministerien, wobei der Gleichstellungsplan als Entscheidungshilfe bei der Führung und der strategischen Planung der Personalpolitik der einzelnen Dienststellen dienen soll.

Mit wenigen Mitteln und geringer Autorität dotiert - und konfrontiert mit der starken Handlungsautonomie der einzelnen Ressorts - stellt sich das BMFSFJ als schwache Koordinierungsinstanz heraus. In diesem institutionellen Kontext hängt die Wirksamkeit der in den Ressorts umgesetzten Instrumente stark von der Leitung jedes Ressorts ab, insbesondere von der Leitung der Personalabteilung (auch „Zentralabteilung“ genannt), die für die Umsetzung des Gleichstellungsgesetzes hauptverantwortlich ist. Diese stark kontextabhängige Umsetzung des Amts der Gleichstellungsbeauftragten und des Gleichstellungsplans wurde in zwei Ministerien untersucht - dem Bundesministerium für Arbeit und Soziales (BMAS) und dem Bundesministerium der Finanzen (BMF) -, die sowohl hinsichtlich des Frauenanteils an ihren Beschäftigten als auch ihres fachlichen Bezugs zur Geschlechtergleichstellung sehr unterschiedliche Konstellationen bieten.

\subsubsection{Bundesfamilienministerium - schwache Koordinierungsinstanz}

Mit 68 Beschäftigten und weniger als $0,6 \%$ des BMFSFJ-Etats, ohne nachgeordnete Behörde oder Ressortforschungseinrichtung kann das Gleichstellungsressort der Bunderegierung ${ }^{12}$ „kaum mehr als den Status eines Feigenblatts beanspruchen", da seine Ressourcen im Verhältnis zu seinen Aufgaben völlig unzureichend sind (Geppert/Lewalter 2012: 7f.). Die Gleichstellungspolitik der Bundesverwaltung ist darüber hinaus nur eine seiner zahlreichen Aufgaben, für die die Kompetenzaufteilung zwischen Gleichstellungsressort und Fachressorts zwar klarer gestellt wird, ${ }^{13}$ die aber genauso unter der schwach ausgeprägten Kultur der interministeriellen Kooperation leidet (ebd.: 6).

Die Bundesministerien tragen die Verantwortung für die Umsetzung des Bundesgleichstellungsgesetzes nämlich selbst (§ 20 Abs. 3 BGleiG). Das

12 Genau betrachtet handelt es sich - wie oben angedeutet - um die Gleichstellungsabteilung des BMFSFJ.

13 Das Gleichstellungsressort hat die Federführung ausschließlich für das BGleiG und das BGremG - in den anderen Aufgabenbereichen ist es mit allen anderen Ressorts formell gleichrangig. 
Gesetz benennt das BMFSFJ lediglich als „Klärungsinstanz“ für die einzelnen Gleichstellungsbeauftragten. Doch zwischen 2004 und 2009 wurde das BMFSFJ nur von einem Viertel der Gleichstellungsbeauftragte konsultiert, und es verfasste in diesem Zeitraum nur vier Rundschreiben und zwei Ergänzungsschreiben zur Umsetzung des BGleiG (Deutscher Bundestag 2010a: 86). Angesichts der Tatsache, dass Informationsdefizite als Grund für die unzureichende Umsetzung des Gesetzes in den Gleichstellungsberichten der Regierung genannt werden, erscheint diese Zahl wie ein Beleg dafür. Hinzu kommt das Faktum, dass die Bundesministerien nicht dem BMFSFJ gegenüber verpflichtet sind, über ihre Fortschritte zu berichten und ihre Gleichstellungspläne weiterzuleiten, weil sie bei ihrer Personalverwaltung weitgehend autonom sind. Beide Umstände deuten nicht nur auf einen schwachen (formalen) Informationsaustausch zwischen dem BMFSFJ und den anderen Ministerien hin, sondern erschweren auch eine systematisch vergleichende Analyse der Gleichstellungspolitik in der Bundesverwaltung.

Dennoch gilt das BMFSFJ als federführendes Ministerium und wirkt als „Wissensvermittler und Informationsgeber“ (Deutscher Bundestag 2010a: 85) vor allem im Rahmen der Berichtspflicht der Bundesregierung gegenüber dem Bundestag. Seit der Einführung des BGleiG muss die Bundesregierung dem Bundestag alle vier Jahre einen „Erfahrungsbericht" über die Umsetzung und die Fortschritte der Gleichstellungspolitik innerhalb der Bundesverwaltung vorlegen $\left(\S 25\right.$ BGleiG). ${ }^{14}$ Das BMFSFJ stützt sich auf diese Rechenschaftspflicht, um den Umfang ihrer Information über die Gleichstellungspraxis der einzelnen Ministerien zu erweitern.

2004 hat das BMFSFJ z.B. für die Vorbereitung des ersten Erfahrungsberichts nach dem neuen Gesetz einen umfassenden und ergänzenden Fragenkatalog an alle betroffenen Dienststellen versandt, weil die Statistiken des Statistischen Bundesamts (obwohl sie seit Einführung des FFG 1994 kontinuierlich erhoben werden) kein vollständiges Bild über den Stand der Frauenförderung und der Förderung der Vereinbarkeit im Bundesdienst wiedergeben konnten. Während alle vorherigen Berichte vom BMFSFJ selbst erarbeitet wurden, wurde für den Berichtszeitraum 2004 bis 2009 ein Forschungsteam für die Erhebung und Analyse quantitativer und qualitativer Daten beauftragt. ${ }^{15}$ Dieses Team führte z.B. eine Interviewkampagne mit den Gleichstellungsbeauftragten der einzelnen Bundesministerien durch und konnte dadurch allgemeine Informationen über den Stand der gleichstellungspolitischen Praxis erheben. In der Öffentlichkeit wurden die einzelnen Bundesministerien nicht benannt aber der Prozentanteil derer, die der Berichtspflicht nachkamen, wurde mit kaum 50\% angegeben (vgl. Abschnitt 5.3.3). Der

14 Nach dem Frauenförderungsgesetz (1994) ist dies in einem Drei-Jahres-Rhythmus vorgesehen.

15 Der Bericht wurde unter der Leitung von Prof. Dr. Gerhard Hammerschmid, einem Experten für Public Management an der Hertie School of Governance (Berlin), erarbeitet. 
Gewinn dieser wissenschaftlichen Evaluation der Gleichstellungspraxis der Bundesverwaltung relativiert sich jedoch dadurch, dass ein aussagekräftiger Vergleich der Praktiken zwischen den Bundesministerien immer noch fehlt. Intern wurde zudem kritisiert, dass die Erfahrungsberichte kaum „Handlungsempfehlungen mit konkreten Zielen, Strategien und Maßnahmen zur Förderung der Gleichstellung" enthalten. ${ }^{16}$

Das BMFSFJ ist außerdem für die Erstellung der Gleichstellungsstatistik der Bundesverwaltung insgesamt verantwortlich. Die 2003 für diesen Zweck verfasste Verordnung ${ }^{17}$ legt eine Liste von zu erhebenden Indikatoren entsprechend einer vorformatierten Tabelle fest. Diese Verordnung wurde 2009 im Rahmen des zweiten Erfahrungsberichts (Deutscher Bundestag 2010a) geprüft, und es wurden Vorschläge für die Einführung neuer Indikatoren formuliert, wie etwa die Verbeamtungsquote nach Geschlecht und Laufbahn sowie Angaben zum leistungsabhängigen Anteil an den Verdiensten (Deutscher Bundestag 2010a: 86). In beiden Fällen geht es um kritische Elemente, die für Geschlechterdiskriminierung anfällig sind..$^{18}$ In den Forschungsaktivitäten und Förderungsprogrammen zur beruflichen Gleichstellung, die vom BMFSFJ initiiert werden, findet man außerdem nur wenige Projekte, die sich auf den Bundesdienst oder die öffentliche Verwaltung beziehen. ${ }^{19}$ Insgesamt verfügt das Gleichstellungsressort über zu wenig statistische Daten und noch weniger qualitative Informationen, um ihre Koordinierungsrolle gezielt ausüben zu können.

\section{Zwischenfazit: Koordinieren und Berichten unter minimalem Zwang}

Als koordinierende Instanz der Gleichstellungspolitik im Bundesdienst besitzt das Gleichstellungsressort (im BMFSFJ) zwar ausdifferenzierte Rechte, sie sind im Gesetz aber so vage formuliert, dass sie der Handlungsautonomie der anderen Ressorts und deren (politischem und haushaltsmäßigen) Gewicht kaum standhalten können.

16 Aus einem nicht öffentlichen Protokoll des Familienausschusses des Bundestags (Protokoll Nr. 17/62 vom 21.03.2012).

17 Verordnung über statistische Erhebungen zur Gleichstellung von Frauen und Männern in Dienststellen des Bundes (Gleichstellungsstatistikverordnung - GleiStatV) vom 18. Juni 2003.

18 Um den Zugang zu aktuellen Daten zum Thema „Frauen in Führungspositionen“ für diese zwei Fallbeispiele zu bekommen, musste ein offizieller Antrag gestellt werden, der in der Rechtsstelle des BMFSFJ geprüft und genehmigt wurde.

19 Siehe etwa das Internetportal zum Thema Wiedereinstieg: http://www.perspektivewiedereinstieg.de. 


\subsubsection{Gleichstellungsbeauftragte in der Bundesverwaltung: Ein ausdifferenziertes Amt}

Das Amt der Gleichstellungsbeauftragten wird in der Literatur als die wichtigste Innovation im jungen Politikfeld der Geschlechtergleichstellung bewertet: Seit 1998 gibt es in allen Bundesländern Gleichstellungsgesetze, die dieses Amt institutionalisiert haben, und Deutschland gilt nun als einziges (europäisches) Land, in dem Gleichstellungsbeauftragte flächendeckend eingesetzt werden (von Wrangell 2012: 20). ${ }^{20}$ Auf internationalen Impuls der Vereinigten Nationen Anfang der siebziger Jahre initiiert und von ausländischen Erfahrungen inspiriert (vor allem in den USA und Schweden), wurde in Deutschland Anfang der achtziger Jahre in von der SPD regierten Städten auf kommunaler Ebene experimentiert, und das Amt wurde erst später auf der Bundesebene eingeführt (Rudolph/Schirmer 2004; von Wrangell 2012). Gleichstellungsbeauftragte der Bundesverwaltung unterscheiden sich von ihren Amtskolleginnen auf Landes- und kommunaler Ebene dadurch, dass sie nur ,nach innen“ arbeiten und am Abbau geschlechtsspezifischer Benachteiligung und Diskriminierung in der öffentlichen Verwaltung durch Frauenförderung im Personalbereich und geschlechtersensible Personalpolitik mitwirken (Schreyögg 2012: 34) ${ }^{21}$ Gemeinsam ist allen Amtsinhaberinnen, dass sie - in Abwesenheit eines klaren Berufsbildes und trotz gesetzlich geregelter Rechte - Position und Einfluss ihres Amtes bei jeder neuen Ernennung erkämpfen müssen.

Funktion mit breit gefächerten Aufgaben: Rechtliche Grundlage

Im Unterschied zu Österreich und zur Schweiz ist die deutsche ministeriale Gleichstellungsbeauftragte nur für ein einziges Diskriminierungsmerkmal das Geschlecht - verantwortlich. Sie fördert und überwacht den Vollzug des Bundesgleichstellungsgesetzes durch ihre Dienststelle, insbesondere in Personalgelegenheiten (Einstellung, Beurteilung, Beförderung, Fortbildung etc.). Sie wirkt auch bei Vereinbarkeitsmaßnahmen mit, wodurch sich ihre Beratungs- und Unterstützungsfunktion auf die männlichen Beschäftigten erstreckt. Ihr Aufgabenbereich wurde durch das BGleiG um die Mitarbeit bei Maßnahmen zum Schutz vor sexueller Belästigung am Arbeitsplatz erweitert.

20 Ihre Zahl lag im Jahr 2003 bei 1.900 Stellen (ohne ehrenamtlich tätige GBAs); ist aber seit Anfang der 1998er-Krise rückläufig und wurde 2011 auf 1.400 geschätzt (von Wrangell 2012: 20; siehe auch Lang 2009; Rudolph 2009).

21 Kommunale GBAs arbeiten auch „nach außen“, indem sie auf eine geschlechtergerechte Gestaltung und Verteilung städtischer Dienstleistungen hinwirken (ebd.). 
In jeder Dienststelle mit regelmäßig mindestens 100 Beschäftigten ist die Wahl einer Gleichstellungsbeauftragte und einer Vertreterin vorgesehen. ${ }^{22}$ Diese werden aus dem Kreis der weiblichen Beschäftigten nach geheimer Wahl durch die weiblichen Beschäftigten für vier Jahre bestellt ( $\$ 19$ Abs. 1 BGleiG nF). Männer dürfen bisher weder an der Wahl teilnehmen noch kandidieren, was jedoch immer öfter hinterfragt wird und auch von den GBAs sehr unterschiedlich bewertet wird. ${ }^{23}$

Gleichstellungsbeauftragte haben grundsätzlich einen Anspruch auf Entlastung von anderweitigen dienstlichen Tätigkeiten von mindestens der Hälfte der regelmäßigen Arbeitszeit bzw. der vollen Arbeitszeit in Behörden mit mindestens 600 Beschäftigten ( $\$ 28$ Abs.2 BGleiG nF). Die Gleichstellungsbeauftragte gehört der Personalverwaltung an, ist aber weisungsfrei in der Ausübung ihrer Tätigkeit. Dieses Amt ist aufgrund der Vermeidung von Interessenkonflikten mit der Mitgliedschaft in einer Personalvertretung unvereinbar.

Die GBA verfügt über ein weitgehendes und frühzeitiges Beteiligungsrecht (z.B. bei der Erstellung des Gleichstellungsplans, bei Personalmaßnahmen). Zur Klärung von Fragen zum BGleiG kann sich die Gleichstellungsbeauftragte unmittelbar (d.h. ohne Einhaltung eines formalen Dienstweges) an das BMFSFJ wenden. Mit dem BGleiG erhielt sie 2001 ein umfassendes Einspruchsrecht (§ 33) und ist damit mit einer starken Kontrollfunktion bei der tatsächlichen Umsetzung der Chancengleichheit von Frauen und Männern im Bundesdienst ausgestattet. Sie verfügt über das Recht, gegen gleichstellungsrelevante Entscheidungen und Maßnahmen ihrer Dienststelle, die sie für rechtswidrig hält, Widerspruch einzulegen, und kann notfalls das Verwaltungsgericht einschalten ( $\$ 34 \mathrm{BGleiG}$ ). Dieser Rechtsweg steht jedoch nur in einigen wenigen sehr restriktiv definierten Situationen offen, etwa wenn die Dienstelle die Rechte der GBA verletzt hat oder wenn kein bzw. ein inhaltlich mangelhafter Gleichstellungsplan vorliegt. Beteiligungsrecht und Einspruchsrecht der GBA wurden mehrmals per Rechtsprechung der Verwaltungsgerichte bestätigt und konkretisiert, so etwa das Recht zur verpflichtenden Teilnahme an Führungsklausuren der Dienststellen (Urteil des BVerwG vom 08.04.2010 - 6 C 3/09) oder an Entscheidungsprozessen bei der Besetzung von Spitzenpositionen (mit ,politischen“ Beamten - Urteile der 5.

22 Mit wachsender Zahl der Beschäftigten (inkl. in nachgeordneten Behörden) ist die Bestellung zusätzlicher Vertreterinnen möglich. Dieses Prinzip wird in der am 06.03.2015 abgeschlossenen Novellierung des BGleiG formalisiert.

23 Die Expertinnen des Deutschen Juristinnenbundes fürchten, dass die Ausweitung der Gleichstellungsarbeit um die „Männerförderung“ durch die Novellierung des BGLeiG diese Wahlregeln angreifbarer machen und die Trennlinie zwischen den Aufgaben der GBA und des Personalrats auflösen würde (Deutscher Juristinnenbund 2014). Die Einführung der Männerförderung wurde in der dem Bundestag vorgelegten Version jedoch zum wesentlichen Teil fallengelassen. 
Kammer VerwG Berlin vom 08.05.2014 - VG 5 K 50.12, VG 5 K 141.12, VG 5 K 412.12).

Uneinheitliche Umsetzung der Rechte der Gleichstellungsbeauftragten: Ein Gesamtbild

Die Gleichstellungsbeauftragte hat ein breit gefächertes Aufgabenspektrum: Sie ist sowohl Förderin, Wissensmanagerin und Beraterin als auch Kontrolleurin bei der Umsetzung des Bundesgleichstellungsgesetzes. Entscheidend für die Aufgabenwahrnehmung ist jedoch die Art und Weise, wie die gesetzlich definierte Beteiligung der GBA an den personellen, sozialen und organisatorischen Maßnahmen, die die Gleichstellung zwischen Männern und Frauen oder die Vereinbarkeit von Beruf und Familie betreffen, tatsächlich umgesetzt wird.

Eine Befragung der GBAs der Bundesverwaltung (im Rahmen des Zweiten Erfahrungsberichts von 2010) ergab, dass dieses Beteiligungsrecht nicht flächendeckend umgesetzt wird und zudem themenabhängig ist: Am häufigsten involviert sind GBAs in Fragen der Personalauswahl oder der Vereinbarkeit, sehr viel seltener allerdings in Fragen der Beförderung oder der Gremienbesetzungen (Deutscher Bundestag 2010a: 81f.). Unterstrichen wird in dem Bericht, dass diese fehlende Beteiligung ,besonders kritisch [gerade] in den Bereichen ist, in denen Benachteiligungen deutlich nachweisbar sind", etwa bei der Höhergruppierung oder der Übertragung von Leitungsfunktionen. Hierfür verantwortlich sei hauptsächlich ein Mangel an Information der Personalverantwortlichen über die Rechte und Zuständigkeiten der GBAs (ebd.: 84).

Interessant ist auch, dass die Gleichstellungsbeauftragten ihre Initiativrechte sehr unterschiedlich nutzen. Am intensivsten werden das Vortragsrecht und das Initiativrecht gebraucht, wohingegen das außergerichtliches Einigungsverfahren oder das verwaltungsgerichtliche Einspruchsrecht kaum zur Anwendung kommt (ebd.: 84). Die Analyse zeigt, dass die GBA ungern einen offenen Konflikt provozieren, da die Wirksamkeit ihrer Arbeit vollständig vom guten Willen der Leitung des Hauses abhängt (ebd.).

Praxis in zwei Ministerien: Eine immer aufs Neue hart erkämpfte Funktion

Aus den Interviews in den beiden Bundesministerien ergibt sich eine allgemeingültige und strukturelle Implikation in der alltäglichen Praxis der GBAs: Über den gesetzlich vorgegebenen Rahmen hinaus ist die konkrete Ausgestaltung der Funktion der Gleichstellungsbeauftragten im Alltag stark umkämpft. Ausstattung und Position müssen in den einzelnen Häusern jeweils hart verhandelt werden und stellen dann die Voraussetzung für die Erfüllung der gesetzlich verankerten Ziele dar. 
Sich in der Hierarchie behaupten: Arbeitsbeziehungen streng definieren

Beide in Mai 2013 interviewten Gleichstellungsbeauftragten mussten sich erst gegenüber der geltenden Hierarchie behaupten. Symbolisch dafür ist die Auseinandersetzung um einen Platz ,oben“ im Organigramm des Bundesministeriums. In der Regel sind die GBAs in der Leitungsebene angesiedelt, genauer bei der Leitung der Personalabteilung (bzw. in der Zentralabteilung). Ein weiterer Aushandlungsgegenstand sind regelmäßige und routinisierte Arbeitsbeziehungen zur Hausleitung. Hierdurch wird ein institutionalisierter Raum geschaffen, in dem gleichstellungsrelevante Fragen unter den Hauptverantwortlichen thematisiert werden können. So wird z.B. das wöchentliche Treffen der GBA mit der Leitung der für Personalfragen zuständigen Zentralabteilung im BMAS als eine gute Einrichtung betrachtet, wenngleich diese Praxis immer wieder bekräftigt und verteidigt werden muss.

Die Tatsache, dass Gleichstellungsbeauftragte nicht als Führungskräfte anerkannt werden, spielt im Informationsaustausch über personalpolitische Angelegenheiten eine Schlüsselrolle: Die GBA gehört de facto nicht zu dem Personenkreis, in dem strategische personalpolitische Orientierungen debattiert werden, wie das Treffen aller Referatsleitungen oder die Führungsklausuren. Trotz gerichtlicher Befassung mit dem Beteiligungsrecht der GBAs, ${ }^{24}$ werden diese nicht systematisch, sondern eher sporadisch zu solchen Treffen eingeladen (Interviews BMAS). Eine Ausnahme bildet hier das Bundesfinanzministerium, in dem die GBA regelmäßig zu den entsprechenden Gremien eingeladen wird. Allerdings wurde hierüber von der GBA einer nachgeordneten Behörde des BMF über einen Zeitraum von sieben Jahren eine gerichtliche Auseinandersetzung erfolgreich bis zur obersten Instanz geführt, womit sich dieses vorbildlichen Verhalten erklären lässt.

Die Vorzimmer-Frage: Kampf um eine angemessene Ausstattung

Weniger strategisch, aber entscheidend für das Alltagsgeschäft einer GBA ist deren materielle Ausstattung. In beiden Bundesministerien wurde die Frage des Vorzimmers, d.h. ob einE MitarbeiterIn zur Verfügung gestellt wird, im Vorhinein ausgehandelt und von der Hausleitung angenommen. Im Fall der Bundesfinanzverwaltung, die mit allen nachgeordneten Behörden 50.000 MitarbeiterInnen und 78 GBAs umfasst, wurde beispielsweise ein MitarbeiterInnenteam von drei Vollzeitkräften eingesetzt, das für das Ministerium und für die Koordinierung der GBAs verantwortlich ist. Nicht nur die Personalausstattung der GBAs bleibt weitgehend (gesetzlich) ungeregelt ${ }^{25}$, hinzu kommt, dass die GBAs bis 2015 über keinen eigenen Haushaltstitel verfüg-

24 Zum Beispiel das - schon erwähnte - Urteil des BVerwG vom 08.04.2010 - 6 C 3/09.

$25 \mathrm{Ab} 1.000$ Mitarbeitern ,ist der Gleichstellungsbeauftragten mindestens eine Mitarbeiterin oder ein Mitarbeiter zuzuordnen“ (§ 29 Abs. 3 des im Jahr 2015 novellierten BGleiG). 
ten, etwa für die Organisation von Informationsveranstaltungen. Vielmehr musste jede Ausgabe einzeln beantragt werden (Interview BMF). ${ }^{26}$

Schlüsselrolle der Information über „Gleichstellung in der Praxis“

Informationsaustausch ist somit das „A und O der Funktion“ der GBA und zwar in beide Richtungen (Interview BMAS): Es geht einerseits darum, der Leitungsebene, den Vorgesetzten und KollegInnen die Funktion der GBA zu erklären. Es geht anderseits aber auch darum, der GBA Informationen aus der Leitungsebene über die strategische Orientierung der Personalpolitik des Hauses zu Verfügung zu stellen. Ein gutes Beispiel hierfür ist die gemeinsame gleichstellungspolitische Reflexion über die jährlich durchgeführte Führungskräftebefragung im BMAS. Herstellung und Erhalt dieser Kommunikationswege ist für die Sichtbarkeit und die Funktionsfähigkeit der GBA zentral.

Wohlbesonnene Kooperation bis zu strategischer Allianz mit dem Personalrat

In keinem der beiden Ministerien entsprechen die Arbeitsbeziehungen zwischen Personalrat und GBA dem aus der Literatur zu erwartenden Muster, nämlich dem der konkurrierenden Funktionen im Bereich Personalpolitik (z.B. Bednarz-Braun 2000: 128). In beiden Fällen hat sich eine gegenteilige Praxis entwickelt: GBAs und Personalräte handeln in einer strategischen Allianz, um das Interesse der MitarbeiterInnen in Sachen Gleichstellung am besten zu vertreten. Dass diese Kooperation nicht selbstverständlich ist, unterstreicht allerdings die Aussage, dass ,diese Zusammenarbeit von der Verwaltung toleriert wird“ (Interview BMF). Strategisch gesehen, erscheint diese Allianz zwischen den BeschäftigtenvertreterInnen als ein Ausgleich für die Mängel der Funktion der GBA, die über kein Vertretungsrecht für benachteiligte MitarbeiterInnen verfügt.

\section{GBA: Karriere oder nur kurzer Karriereabschnitt?}

Das Amt der GBA kann von den gewählten Beschäftigten sehr unterschiedlich in die eigene Laufbahn integriert werden. Eine GBA kann ihr Amt entweder als einen kurzen Abschnitt ihrer Karriere oder als eine neue Karriere wahrnehmen. Im zweiten Fall beabsichtigt sie ein längeres Verbleiben im Amt. Beide Varianten sind mit unterschiedlichen Risiken verbunden. In beiden Sichtweisen kann aber die Funktion der GBA einem „Karriereknick“ gleichkommen. Als erster Grund dafür wird die starre Statusregel der Funk-

26 Die am 06.03.2015 abgeschlossene Novellierung des BGleiG ändert es marginal: Eine MitarbeiterIn ab 1.000 Beschäftigten und ein Verfügungsfonds von 26 Euro pro Monat sind vorgesehen. Beide Maßnahmen wurden von der IMA als völlig unzureichend betrachtet (Rose-Möhring 2015: 6). 
tion genannt: Ein beruflicher Aufstieg durch das Amt ist schwierig - man tritt in die Funktion ein und scheidet mit dem vorherigen Status (Sachbearbeiterin oder Referentin, je nach dem) wieder aus. Zudem werden die im Amt gewonnenen beruflichen Kompetenzen (Konfliktmanagement, Personalführung etc.) nur begrenzt als aufstiegsrelevante Leistungskriterien anerkannt. Für das zweite Profil, bei dem die GBA lange im Amt verbleibt, birgt dieses Amt ein zusätzliches Risiko: Abgewählt zu werden, kann zu einer persönlich schwierigen Situation führen und als Statusverlust empfunden werden, selbst wenn eine „fiktive Nachzeichnung“ des beruflichen Werdegangs (§ 28 Abs. 3 BGleiG $\mathrm{nF}$ ) vorgesehen ist. Zwar werden anhand einer Vergleichsgruppe vergangene Beurteilungen (d.h. vor der Freistellung zur GBA) nachgezeichnet, eine formale Anerkennung der im Amt spezifischen fachlichen Qualifikationen und Kompetenzen würden die verschiedenen, jedoch offensichtlichen Nachteile des Amtes jedoch sehr viel angemessener kompensieren und $\mathrm{zu}$ einer besseren Verbindung von Laufbahn und temporärer Funktion führen. ${ }^{27}$

\section{Ressortübergreifende Vernetzung der GBA im IMA}

Bemerkenswert ist, dass die GBA im Bundesdienst (816 Frauen insgesamt, inkl. nachgeordnete Behörden) sehr wenig systematisch kooperieren. Der auf 24 GBAs begrenzte interministerielle Arbeitskreis der Gleichstellungsbeauftragten des obersten Bundesdienstes (IMA) besitzt kaum Sichtbarkeit. Der IMA besteht seit 1991 und wurde auf Initiative der damaligen Frauenministerin (Angela Merkel) gegründet. Dank der Weisungsfreiheit seiner Mitglieder setzt er seine Arbeitsschwerpunkte und Gleichstellungsakzente selbst. Seine Aufgaben sind, wenngleich nirgendwo offiziell definiert, ,gemeinsame Ziele zu entwickeln, Gespräche mit einflussreichen Persönlichkeiten zu Gleichstellungsfragen zu führen und Verbesserungen auch bei einzelnen Rechtsfragen zu erreichen“. ${ }^{28}$ Da die Arbeit des IMA ,nach innen“" in die Bundesministerien gerichtet ist, wird ihr keine Öffentlichkeitsarbeit gestattet - wie es auch der Fall bei den einzelnen GBAs ist. Die Beziehung zum Parlament wird ebenfalls als defizitär beschrieben: Bislang haben zwei Anhörungen des IMA im Rahmen einer Sitzung eines Bundestagausschusses stattgefunden (Inter-

27 In einer Stellungnahme zum Thema empfiehlt das BMI 2010 auch in diesem Sinne: „Vielmehr ist im Rahmen der fiktiven Nachzeichnung individuell an den Leistungsstand vor der Amtsübernahme anzuknüpfen, der je nach erreichtem Niveau unter Berücksichtigung der üblicherweise durch eine Fortsetzung der Arbeit erworbenen zusätzlichen Fähigkeiten fortzuschreiben ist“" (http://www.bmfsfj.de/BMFSFJ/gleichstellung,did=164566.html; Zugriff: 26.10.2015).

28 Aus dem nicht offiziellen Protokoll einer entsprechenden Anhörung im Bundestag (siehe Fn. 29). 
view BMF). ${ }^{29}$ Die große Koalition hat aber im Rahmen der Novellierung des BGleiG diesen Arbeitskreis mit einer gesetzlichen Grundlage ausgestattet (BMFSFJ 2014). Hierdurch wurde die Vernetzung der ministerialen GBAs und ihre Position als fachliches Gremium bestätigt.

\section{Zwischenfazit: Ausdifferenzierte Rechte, kaum Sanktionen bei Missachtung}

Die Funktion der Gleichstellungsbeauftragten in den obersten Bundesbehörden wurde zwar im Lauf der Zeit durch neue gesetzliche Regelungen weiter gestärkt, ${ }^{30}$ ihre faktischen Einflussmöglichkeiten sind in der ministerialen Praxis jedoch eine hart erkämpfte Angelegenheit - von der Ausgestaltung jeder einzelnen MitarbeiterInnenstelle bis zur gerichtlichen Erstreitung präziserer Beteiligungsrechte und der Mitwirkung an strategischen personalpolitischen Entscheidungen. Hiermit erweist sich die gesetzliche Ausgestaltung des Amtes der GBA als relativ ausdifferenziert hinsichtlich der Definition ihrer Rechte, wenngleich eine genaue Festlegung der notwendigen Ressourcen und Beteiligungsrechte die reale Machtposition der GBA klar definieren und Konflikte über deren Aushandlung vermeiden könnte. Ihr restriktives Einspruchsrecht gilt als Merkmal einer minimalen Verbindlichkeit. Die Nichteinhaltung der Rechte der GBA wird kaum kontrolliert und noch seltener angefochten. Das Verbot jeder Öffentlichkeitsarbeit schränkt die Verbindlichkeit der Mitwirkungsrechte zusätzlich ein. Dennoch spielt die Rechtsprechung eine wesentliche Rolle in der Spezifizierung und der Erweiterung der Geltungsbereich der Rechte der GBA. Allerdings wird die Erweiterung der Reichweite der Institution GBA durch langwierige und karrierepolitisch riskante Prozesse letztlich von einzelnen Personen individuell erkämpft.

29 Bei der ersten (nicht öffentlichen) Anhörung ging es um ein „Gespräch mit der Vorsitzenden und zwei weiteren Mitgliedern des Interministeriellen Arbeitskreises der Gleichstellungsbeauftragten der obersten Bundesbehörden (IMA) über den Stand der Gleichstellung und die Entwicklungschancen für Frauen " in den Bundesministerien. Dieses Gespräch fand während der 62. Sitzung des Ausschusses für Familie, Senioren, Frauen und Jugend am 21.03.2012 statt. Zu dem Gespräch existiert jedoch kein öffentlich zugängliches Protokoll. Die zweite Anhörung fand am 23.02.2015 im Rahmen der Beratung zur Novellierung des BGleiG statt und war öffentlich (siehe Rose-Möhring 2015).

30 Eine wesentliche Ausnahme bildet in dieser Hinsicht die in März 2015 durchgeführte Novellierung des BGleiG - die von manchen ExpertInnen eher als ein „Bärendienst für die Gleichstellung im Bund“ betrachtet wird (http://www.rehmnetz.de/gleichstellungsrechtblog/trauer-muss-die-gleichstellung-tragen/; Zugriff: 26.10.2015), da sie die Rechte der GBAs kaum erweitert und zum Teil schwächt. 


\subsection{3 „, Gleichstellungscontrolling“ durch Gleichstellungspläne: Eine Führungsfrage}

Gleichstellungspläne gehören zu den klassischen Instrumenten betrieblicher Gleichstellungspolitik (Bothfeld et al. 2010: 41f.): Auf die Veränderung betrieblicher Strukturen zielend, dienen Gleichstellungspläne der Erfassung und Offenlegung von Geschlechterungleichheiten in der Beschäftigungsstruktur einer Organisation. Sie fordern somit den Arbeitgeber zu gleichstellungspolitischen Aktivitäten auf, um diese Ungleichheiten zu beheben. Eine solche Planerstellungspflicht gilt für die obersten Bundesbehörden seit dem Frauenfördergesetz (1994).

Strategisches Instrument zu personalpolitischen Zwecken

Die Gleichstellungspläne sind von sämtlichen Dienststellen des Bundes alle vier Jahre zu erstellen ( $\$ 12$ BGleiG $\mathrm{nF}$ ). Zum einen wird - wie im früheren FFG - eine Situationsbeschreibung der Personalstruktur der Dienstelle anhand einer geschlechterdifferenzierenden quantitativen Darstellung und einer qualitativen Auswertung der bisherigen Förderungsmaßnahmen erstellt, um einen systematischen Zusammenhang zwischen Fördermaßnahmen und zeitlichen Variationen in der Personalstruktur (vor allem Frauenanteile in den einzelnen Bereichen) abzubilden. Kernstück des Gleichstellungsplans sind dann konkrete und verbindliche Zielvorgaben zur Beseitigung der Unterrepräsentanz von Frauen in allen Bereichen, die mit einem Maßnahmenkatalog und einem zeitlichen Umsetzungsplan zu verbinden sind. Vorgesehen ist die Besetzung mindestens der Hälfte der offenen Mitarbeiterstellen mit Frauen in allen Bereichen, in denen Frauen unterrepräsentiert sind. Diese Quotierung bindet die Dienststelle nicht unmittelbar bei der Besetzung einer bestimmten Stelle, jedoch ist die Nichterfüllung der Zielvorgaben gegebenenfalls nach zwei Jahren bzw. im darauffolgenden Plan zu begründen. Ein fester Zeitraum zur Erfüllung der Quote wird im Gesetz jedoch nicht benannt.

Der Gleichstellungsplan gilt laut Gesetz als strategisches Instrument der Personalplanung und der Personalentwicklung ( $§ 11$ BGleiG), da im Zuge der Verwaltungsmodernisierung das BGleiG die Integration von Personalpolitik und Gleichstellungspolitik vorsieht (Schiek et al. 2002: 133, 169). Seine Umsetzung ist deswegen nicht nur für die Personalverwaltung der einzelnen ministeriellen Ressorts verpflichtend, sondern auch für alle Vorgesetzten (z.B. ReferatsleiterInnen).

Der Gleichstellungsplan unterliegt einer Veröffentlichungspflicht, die sich jedoch nur auf die interne Öffentlichkeit der Dienstelle bezieht. Die Begründungspflicht gilt ebenfalls nur nach innen. Gegen eine unzureichende, fehlende oder nicht rechtzeitige Umsetzung des Gleichstellungsplans kann laut 
Gesetz die Gleichstellungsbeauftragte Einspruch erheben. Auch ist die GBA frühzeitig an der Erstellung des Gleichstellungsplans zu beteiligen.

Bemerkenswert ist, dass bisher kein Leitfaden zur Erstellung des Gleichstellungsplans durch das BMFSFJ zu Verfügung gestellt wurde. Das Gesetz sieht auch bisher keine Sammlung, Übersicht oder Auswertung der Gleichstellungspläne der Gesamtheit der Dienststellen der Bundesverwaltung vor. Ohne eine zentralisierte Kontrolle und ohne jede externe Sanktionsmöglichkeit bei Nichterfüllung umgehen die Bundesministerien leichthin diese gesetzliche Verpflichtung. Die im März 2015 abgeschlossene Novellierung des BGleiG füllt diese Lücke jedoch in keiner Weise.

\section{Von unkoordinierter Erstellung bis zu ineffektiver Umsetzung}

Dementsprechend gibt es keine vergleichende Auswertung der Gleichstellungspläne, aus der sich Hinweise auf gute Praktiken ablesen ließen, auch nicht durch das BMFSFJ als koordinierende Instanz. Der zweite Erfahrungsbericht enthält vielmehr klare Hinweise auf ein allgemeines Umsetzungsdefizit: 2010 hatten nur etwas über die Hälfte der Personalreferate einen Gleichstellungsplan erstellt, zudem wurden viele der Pläne nicht aktualisiert. Obwohl die inhaltliche Ausgestaltung des Gleichstellungsplans gesetzlich geregelt ist, enthielt nur die Hälfte der Pläne konkrete Maßnahmen und Zielvorgaben; in über zwei Drittel der Pläne fehlte außerdem ein Zeitplan für die Realisierung der Maßnahmen, obwohl schon im vorherigen Berichtszeitraum (2004-2009) die Zielvorgaben in mehr als der Hälfte der Fälle nicht erreicht worden waren. Aus diesem Grunde wird die Wirksamkeit der Gleichstellungspläne als Instrument der Steuerung der beruflichen Gleichstellung in der Bundesverwaltung von den GBAs als insgesamt gering eingeschätzt (Deutscher Bundestag 2010a: 89-92).

Führung der Personalpolitik als Angelpunkt

Im Rahmen dieser empirischen Untersuchung offenbarte sich ein sehr unterschiedlicher Umgang mit dem Gleichstellungsplan. Während im BMAS die Broschüre des aktuellen Gleichstellungsplans und später die elektronische Version des vorherigen Plans sowie in beiden Fällen ihre Überarbeitung gut zugänglich waren, wird der Gleichstellungsplan des BMF sehr strikt nur intern verwandt. Als strategisches Instrument zur Planung, Steuerung und Kontrolle der gleichstellungsrelevanten Aspekte der Personalpolitik in der jeweiligen Behörde wird die Planpflicht somit sehr unterschiedlich gehandhabt und umgesetzt.

Im BMAS wurden die zwei letzten Gleichstellungspläne (2006-2010, 2010-2014) musterhaft umgesetzt: Sie enthalten eine (statistisch untermauerte) Situationsbeschreibung, eine kurze Auswertung der Ursachen für die Unterrepräsentanz von Frauen sowie eine umfassende Übersicht über die 
Ergebnisse der in der Vergangenheit ergriffenen Maßnahmen sowie auch neue, teilweise quantifizierte Zielvorgaben und schließlich die entsprechenden Maßnahmen zum Erreichen dieser Ziele. Der Gleichstellungsplan gilt hier als wirksames Instrument, weil er im Rahmen eines kontinuierlichen Monitorings durch die Zentralabteilung des BMAS erstellt wird (Interview BMAS). Die enthaltenen personalpolitischen Statistiken werden regelmäßig aktualisiert und bei Einstellungs-, Beförderungs- und Beurteilungsprozessen von der Personalverwaltung ,im Auge behalten“ und als Orientierungsmaßstab bei den betroffenen Entscheidungen angewandt (ebd.).

Im Bundesfinanzministerium wird dem Gleichstellungsplan eine untergeordnete Rolle zugeschrieben: Die Personalverwaltung erfüllt ihre gesetzliche Berichtspflicht, indem sie sich zu bestimmten Maßnahmen und $\mathrm{zu}$ nicht quantifizierten Ziele bekennt. Sie verkündet lediglich ,ihre Bemühung“, sich diesem Ziel zu nähern (Interview BMF). Somit spielt der Gleichstellungsplan keine wesentliche strategische Rolle in übergeordneten personalpolitischen Planungsprozessen.

Die Art der Nutzung des Gleichstellungsplans variiert zwischen personalpolitischem Steuerungsinstrument und formaler Regelerfüllung - im zweiten Fall, ohne dass strategische oder personalpolitische Folgen abgeleitet werden. Als erklärender Faktor für diesen Unterschied wird das geschlechterpolitisch sensible Profil der Leitung der Personalabteilung der Dienstelle herangezogen: Im Fall des BMAS wurde die Leiterin der Personalabteilung u.a. Mitglied des für Gender Mainstreaming verantwortlichen Arbeitsstabes im Kanzleramt (Interview BMAS).

Zwischenfazit: Der Gleichstellungsplan - ein verbesserungsbedürftiges Instrument

Die Verbindlichkeit des Gleichstellungsplans wird im Gesetz minimal definiert: Seine Umsetzung wird von keiner zentralen Instanz kontrolliert oder sanktioniert; er muss lediglich innerhalb der einzelnen Ministerien veröffentlicht werden. Allerdings kann die GBA gegen seine gesetzwidrige Umsetzung Einspruch einlegen. Da es keine einheitliche vorgegebene Struktur für den Gleichstellungsplan gibt - nur die Angabe von quantifizierten Zielen und Fristen wird gefordert -, bleibt seine Ausdifferenziertheit im Gesetz begrenzt. Obwohl seine Reichweite anspruchsvoll ist - grundsätzlich soll er auf jeder Führungsebene bis hin zum Referat umgesetzt werden -, sind die Wirkungsvoraussetzungen des Instruments insgesamt unzureichend.

Dennoch glauben die im BMFSFJ für Gleichstellungspolitik verantwortlichen Personen, dass eine breite Veröffentlichung der Gleichstellungspläne der direkteste und wirksamste Weg sei, die Bundesministerien zur Umsetzung der Gleichstellung zu aktivieren (Interview BMFSFJ). Eine solche Veröffentlichung, selbst wenn sie nur innerhalb der Bundesverwaltung erfolgte, würde die einzelnen Ministerien dazu ermutigen, aktiver zu werden, um nicht 
als Nachzügler zu erscheinen. Ein „Ranking“ der einzelnen Bundesministerien anhand zentraler gleichstellungsrelevanter Daten und Maßnahmen ${ }^{31}$ und die Veröffentlichung des Rankings im Rahmen des Erfahrungsberichts wäre dann die ideale Ergänzung zum Gleichstellungsplan. Durch diese Transparenz wären die einzelnen Bundesministerien gezwungen, aufgrund eines „blaming and shaming“-Effekts zu agieren (Bothfeld/Rouault 2015). Um dies durchzusetzen, bedarf es ohne Zweifel der Unterstützung des Innenministeriums, das die Federführung für die Personalpolitik im Bundesdienst innehat; das in der Vergangenheit jedoch eher durch die Verzögerung weiterer Maßnahmen als durch aktive Förderung der Gleichstellung in der Bundesverwaltung in Erscheinung getreten ist (Interview BMAS).

Tatsächlich sah der erste Entwurf für die Novellierung des BGleiG vor, die Gleichstellungspläne der Bundesministerien künftig zu veröffentlichen, um Transparenz zu schaffen, eine bessere Kontrolle zu ermöglichen und den „Wettbewerb als attraktiver Arbeitgeber“ zu fördern. Die Veröffentlichung der Gleichstellungspläne sollte durch einen Gleichstellungsindex flankiert werden, der die wichtigsten Gleichstellungsindikatoren der obersten Bundesbehörden jährlich abbilden und vergleichbar machen soll (BMFSFJ/BJV 2014: 10). Die am 06.03.2015 beschlossene Novellierung beschränkt sich jedoch auf die Erstellung des Index und enthält die Veröffentlichungspflicht nicht mehr - ein Beleg für die starken Widerstände innerhalb der Bundesverwaltung gegen eine größere Transparenz.

\subsection{Substanzielle Gleichstellungspolitik in der deutschen Bundesverwaltung: Auf der Suche nach dem kleinsten gemeinsamen Nenner}

Die Vereinbarkeit von Beruf und Familie und die Förderung von Frauen in Führungspositionen stellen den Kern der gleichstellungspolitischen Aktivitäten der untersuchten Bundesministerien dar. Beide Ansätze konnten bislang nur in recht unterschiedlichem Maße mit der Personalpolitik der Bundesverwaltung verknüpft werden: Während die Instrumente zur Vereinbarkeit sich in hohem Maß diversifizieren, bleiben die Instrumente zur Karriereförderung von Frauen spärlich und ungenutzt. Die unterschiedliche Gewichtung (gemessen an der Diversität und Inanspruchnahme der Maßnahmen) dieser zwei Kernthemen spiegelt sowohl die thematischen Prioritäten der vorherigen konservativ-liberalen Bundesregierung als auch die fortgesetzte Fragmentie-

31 Ein Ranking schließt eine Gewichtung und die Addition einzelner gleichstellungspolitisch relevanter Indikatoren ein. Zwar wurden diese im letzten Erfahrungsbericht nach Ressort (Anhand der Gleichstellungstatistik) erhoben, waren aber nur in Form einer unkommentierten Tabelle zu finden (Deutscher Bundestag 2010a: 101). 
rung des deutschen Geschlechterregimes durch die als prioritär eingestufte geschlechterrollenbezogene Familienpolitik wider (Bothfeld 2008).

\subsubsection{Breit gefächerte Instrumente der Vereinbarkeit von Beruf und Familie im Bundesdienst}

Die drei Hauptinstrumente der Vereinbarkeitspolitik im Bundesdienst sind dieselben wie in der Privatwirtschaft, jedoch mit einer anderen Gewichtung. Das am weitesten verbreitete und am längsten erprobte Instrument ist die Teilzeitarbeit, während die sog. ,mobile Arbeit" erst an Bedeutung gewinnt, wohingegen sich die Frage der Kinderbetreuung im Berliner Kontext als weniger zentral erweist.

\section{Universaler, aber ambivalenter Anspruch auf Teilzeit}

Die Inanspruchnahme von Teilzeitarbeit hat gerade im Bundesdienst in den vergangenen Jahren aufgrund der wachsenden Frauenbeschäftigung zugenommen und sich über alle Funktionsebenen hinweg in ihrem Umfang angeglichen (siehe Abschnitt 5.2). Die hohe Akzeptanz dieses Instruments erklärt sich auch aus der zunehmenden Inanspruchnahme durch hoch qualifizierte Frauen (Deutscher Bundestag 2010a: 62). Diese Praxis steht in einem starken Kontrast zur Privatwirtschaft, in der der Umgang mit den Teilzeitrechten der Beschäftigten - besonders bei Führungskräften - restriktiv bleibt (Koch 2008).

Teilzeitarbeit spielt nämlich in der Geschichte der Geschlechtergleichstellung im öffentlichen Dienst eine Schlüsselrolle, nachdem das Teilzeitverbot für Beamtinnen im Bundesdienst 1969 aufgehoben wurde (für männliche Beamte 1971). Zuvor mussten Frauen nach der Mutterschutzfrist aus dem öffentlichen Dienst ausscheiden, wenn sie keine Kinderbetreuung fanden, die ihnen eine vollzeitige Tätigkeit ermöglichte. Über die Aufhebung des Teilzeitverbots wurde mehr als drei Jahre lang im Bundestag verhandelt (von Oertzen 1999b). Mittlerweile gilt das Beamtenrecht bei der Gewährung von Flexibilität als vorbildlich (Schiek et al. 2002: 223), vor allem hinsichtlich der Möglichkeiten, die Arbeitszeit individuell an die Bedürfnisse der MitarbeiterInnen anzupassen - durch Festsetzung des Teilzeitvolums, Gleitzeit sowie Kontingente für mobile Arbeit. Die Gleichstellungsgesetzgebung hat zu dieser Entwicklung wesentlich beigetragen. Durch das Bundesgleichstellungsgesetz (2001) haben auch die Beschäftigten mit Vorgesetzten- und Leitungsaufgabeneinen Anspruch auf Teilzeitbeschäftigung (§ 16 Abs. 1 BGleiG $\mathrm{nF})$. 
Heute können nämlich alle BeamtInnen Teilzeitarbeit ${ }^{32}$ beantragen, aber es sind verschiedene Kategorien zu unterscheiden: der „normale“ Antrag (§ 91 BBG), der Antrag aus familiären Gründen (§ 92 BBG) oder auf Altersteilzeit ( 993 BBG). Im ersten Fall muss die Teilzeitbeschäftigung mindestens die Hälfte der regulären Arbeitszeit betragen. Der Antrag kann nur abgelehnt werden, wenn ihm dienstliche Belange entgegenstehen. Die familiäre Teilzeitbeschäftigung kann in Anspruch genommen werden, wenn der/die Betroffene mindestens ein Kind unter 18 Jahren oder eine/n pflegebedürftige/n sonstige/n Angehörige/n betreut. In diesem Fall hat der Gesetzgeber die Hürde für eine Ablehnung höher gelegt: Es müssen zwingende dienstliche Belange entgegenstehen. Die Höhe der Arbeitszeit kann hier auch weniger als die Hälfte der regulären Arbeitszeit betragen. Teilzeitarbeit wird dann jedoch längstens bis zu einer Dauer von 15 Jahren gewährt.

Teilzeitbeschäftigte müssen tatsächlich dienstlich entlastet werden, und Ihnen sind zudem Telearbeitsplätze oder besondere Arbeitszeitmodelle (Sabbatjahr, Arbeitszeitkonten) anzubieten. Aufgrund der Fürsorgepflicht des Dienstherrn müssen die Beschäftigten über die Folgen dieser Teilzeitbeschäftigung informiert werden - seien es beamten-, arbeits-, versorgungs- oder rentenrechtliche Folgen oder die Möglichkeit einer befristeten Gewährung der Teilzeitarbeit mit der Option einer Verlängerung. Im Fall einer unbefristeten Teilzeitstelle gibt es nämlich keinen unbedingten Anspruch auf Rückkehr zu einer Vollzeitbeschäftigung. Aus einer Geschlechtergleichstellungsperspektive sind einige dieser Reformen daher ambivalent. So wurde z.B. im Fall des Teilzeitantrags aus familiären Gründen der Mindestumfang der wöchentlichen Arbeitszeit, der vorher bei 50\% der regulären Wochenarbeitszeit lag, abgeschafft.

Problematisch ist daher die fehlende statistische Erfassung des Stundenumfangs der Teilzeitbeschäftigungsverhältnisse, die eine genauere Analyse des Instruments verhindert, z.B. bei der Frage, ob es möglicherweise eine Tendenz zu längeren Teilzeitformen nach dem ,30-Stunden-Modell““ gibt. Das Fehlen der Daten ist umso kritischer zu bewerten, weil Teilzeitarbeit ein anerkanntes Hauptmerkmal bei der Benachteiligung von Frauen im Rahmen der Beurteilungsprozesse sowie der eng damit verkoppelten Benotungs- und Beförderungsprozesse darstellt: Während 4,9\% der Vollzeitkräfte im Durchschnitt zwischen 2004 und 2009 befördert oder höhergruppiert wurden, waren es bei den Teilzeitbeschäftigten nur 2,1\% (Deutscher Bundestag 2010a: 64).

Die Bewertung von Teilzeitarbeit stellt bei Beurteilungsprozessen ein von den beteiligten Akteuren anerkanntes Problem dar. In den ressortspezifischen Vereinbarungen zu Thema Beurteilung ${ }^{33}$ wird zwar ein Benachteiligungsver-

32 Im Bundesdienst beträgt die reguläre Wochenarbeitszeit 41 Stunden.

33 Auch wenn das BMI den Ministerien Anleitungen für die Durchführung von Beurteilungsprozessen liefert, ist die konkrete Ausgestaltung der Beurteilungssysteme jedem Ministe- 
bot wegen Teilzeitbeschäftigung formuliert, aber die Gründe einer möglichen Diskriminierung erweisen sich als schwer identifizierbar, weil sie als kultureller Natur diagnostiziert werden. So wird die Arbeitsleistung und -bereitschaft mehr oder weniger unbewusst in Abhängigkeit von Präsenz bzw. Dienstbefreiung bewertet, sodass eine relative Abwertung von Teilzeitarbeit oder mobiler Arbeit unausweichlich ist. ${ }^{34}$ Der Einsatz eines Zweitbeurteilenden und die Herstellung eines Bewertungsspiegels (mit gesondertem Anteil von Männern und Frauen, Teilzeitbeschäftigten und flexibel Arbeitenden) sind zwei weiteren Maßnahmen, die eingesetzt werden, um diesem Diskriminierungspotenzial entgegenzuwirken. Auch wenn in manchen Bundesministerien entsprechende Schulungen für Führungskräfte angeboten werden, bei denen die Gestaltung vorurteilsfreier Beurteilungsprozesse vermittelt werden soll, wird das Fehlen eines einheitlichen Standards bei solchen Beurteilungsprozessen zwischen den Bundesministerien bemängelt (ebd.: 63f.).

\section{Kinderbetreuung: Keine Priorität dank dem DDR-Erbe}

Im Bereich Kinderbetreuung werden widersprüchliche Ansichten vertreten. Im Erfahrungsbericht von 2010 wurde durch die Gleichstellungsbeauftragten noch ein Mangel an Initiative auf Seiten der Bundesministerien bedauert (Deutscher Bundestag 2010a: 71). Im Sommer 2013 berichteten die MitarbeiterInnen jedoch von einer relativ großen Zufriedenheit mit der Situation (Interview BMAS). Die Unzufriedenheit mit den Bedingungen der Kinderbetreuung läge eher an den hohen Ansprüchen der Eltern; die hohe Zufriedenheit mit den Betreuungsmöglichkeiten insgesamt hingegen sei eher mit dem „Berliner Erbe“ zu erklären (Interview BMAS). Tatsächlich ist das Angebot an externen Betreuungsplätzen in Berlin überdurchschnittlich hoch, während die Situation der MitarbeiterInnen in Bonn eher unbefriedigend sei (ebd.). Das als Notlösung konzipierte „Eltern-Kind-Zimmer“35 wird in beiden Ministerien kaum in Anspruch genommen, da sich inzwischen eine hohe Toleranz für die sporadische Anwesenheit von Kindern am Arbeitsplatz entwickelt hat (Interview BMAS/BMF).

rium überlassen. So schließt jedes Haus mit dem Personalrat eine Dienstvereinbarung über die geltende Beurteilungsrichtlinie ab und entwickelt dann einen praxisorientierten Leitfaden zur Erstellung der Beurteilungen.

34 Die Elemente dieser Diagnose stammen aus der Vereinbarung und dem Leitfaden eines Bundesministeriums, das nicht Gegenstand dieser Studie ist, das aber als vorbildlich für seine Gleichstellungspolitik gilt. Vergleichbare Zusammenhänge sind aber aus der Interviews im BMAS und in deren Vereinbarung zu finden.

35 In beiden Bundesministerien wird ein Arbeitszimmer mit Spielecke für solche Notfälle zu Verfügung gestellt. 
Zentraler Stellenwert der mobilen Arbeit

Auf der Vereinbarkeitsagenda der Bundesverwaltung kommt der Flexibilisierung des Arbeitsortes eine zentrale Bedeutung zu: Telearbeit ermöglicht abwechselnde Arbeitsplätze in einem festgesetzten wöchentlichen Rhythmus, während mobile Arbeit eine sowohl örtliche als auch zeitliche Flexibilität ermöglicht. Beide Formen der flexiblen Arbeitsgestaltung tragen zur progressiven Schwächung der für viele Benachteiligungen verantwortlich gemachten „Präsenzkultur“ bei.

Nach Erprobung des Modells der Telearbeit, das oft auf MitarbeiterInnen mit Familienpflichten begrenzt war, wird derzeit in beiden untersuchten Ministerien das Modell „mobile Arbeit" durch Dienstvereinbarungen eingeführt. Mobile Arbeit richtet sich an alle MitarbeiterInnen und zielt langfristig auf eine volle Flexibilisierung des Arbeitsortes. Vereinbarungen zur mobilen Arbeit werden individualvertraglich auf Antrag getroffen. Die Spielräume für die Anwesenheitspflicht am Arbeitsplatz sind weit gefasst: So legt die Dienstvereinbarungen z.B. im BMF lediglich fest, dass alle MitarbeiterInnen an einem bestimmten Tag der Woche im Ministerium gleichzeitig anwesend sein sollen. Die Vereinbarungen unterscheiden zwischen privaten Zwecken und dienstlichem Interesse an mobiler Arbeit; das BMF sieht z.B. ein Kontingent von 200 Stunden pro Jahr für private Zwecke vor. Für MitarbeiterInnen in Führungspositionen (ab Besoldungsgruppe B6 bzw. Unterabteilungsleitungen) gilt die Vereinbarung im BMF allerdings nicht.

Mittelfristig richtet sich mobile Arbeit nach den Präferenzen der kommenden Generation von MitarbeiterInnen (der sog. „Generation $Y^{\prime)}$, die sich von der „Präsenzkultur“ im öffentlichen Dienst distanziert (Interview BMF). Die Politik des flexiblen Arbeitsortes nimmt in diesem Fall starke antizipative Züge an.

Neue Tendenzen entlang der neuen Beschäftigtenpräferenzen?

In beiden untersuchten Ministerien zeichnen sich nach Angaben der Interviewten neue Tendenzen bei der Umsetzung der Vereinbarkeitspolitik ab, die statistisch jedoch nicht erfasst werden. Unter den jüngeren MitarbeiterInnen verbreiten sich sowohl längere Teilzeitmodelle (mit 30 Stunden Wochenarbeitszeit) als auch die vorzeitige Rückkehr aus dem Elternurlaub. Jüngere Männer machen nun auch mehr Gebrauch von vereinbarkeitspolitischen Maßnahmen, vor allem jedoch durch die wachsende Inanspruchnahme der Partnermonate der Elterngeldregelung. 


\subsubsection{Vereinbarkeit von Beruf und Familie oder Reduktion von Gleichstellungspolitik auf konsensuale Maßnahmen}

Wie erklären die Akteure in den zwei Bundesministerien die Zentralität der Vereinbarkeitsfrage in der Gleichstellungspolitik und ihre breite Akzeptanz, sowohl bei den Führungskräften als auch bei den Beschäftigten beider Geschlechter?

Familienfreundlichkeit als Pflicht: Audit „berufundfamilie“

Die hohe politische Priorität, die der Vereinbarkeitspolitik zugeschrieben wird, spiegelt sich in dem Kabinettbeschluss vom 20. August 2008 wider, der als verbindlich vorsah, dass sich alle Bundesministerien bis Ende 2009 dem Audit berufundfamilie der Hertie-Stiftung unterziehen und sich als familienbewusste ArbeitgeberInnen zertifizieren lassen sollen (Deutscher Bundestag 2010a: 57). Mittlerweile haben einige Bundesministerien schon die dritte Runde der Zertifizierung durchlaufen.

Das Audit berufundfamilie soll als strategisches Managementinstrument der Verbesserung einer familienbewussten Personalpolitik dienen. Es wurde vor 15 Jahren aus der Initiative der gemeinnützigen Hertie-Stiftung entwickelt. ${ }^{36}$ Beide untersuchten Ministerien sind schon durch zwei (BMF) bzw. drei (BMAS) Audit-Runden gegangen. Wenngleich die Auszeichnung als ein wenig direkt wirksames, eher nach außen orientiertes Werbeinstrument betrachtet wird, gilt der Austauschprozess unter Mitgliedern der thematischen Arbeitsgruppen doch als wichtiger Raum, in dem die Vereinbarkeitspolitik des Hauses reflektiert und weiterentwickelt wird (Interviews BMAS/BMF). Allerdings wird der Audit-Prozess selbst (d.h. die Vorbereitung des Berichts an die Gutachterkommission) als komplex, langwierig und nicht immer konfliktfrei beschrieben (ebd.).

\section{Hohes Konsenspotenzial der Vereinbarkeitspolitik}

Für die überragende Bedeutung der Vereinbarkeitspolitik für die Gleichstellungsproblematik lassen sich drei zusätzliche Argumentationsmuster identifizieren. Das Motto „man bietet allen [d.h. Männern und Frauen] etwas an niemandem wird etwas weggenommen" (Interview BMAS) betont die konsensuale Kraft der Vereinbarkeitsthematik der Gleichstellungspolitik. Das Thema passt sowohl zu den ,traditionellen Einstellungen der älteren männlichen Führungskräfte“ als auch zu den moderneren Erwartungen jüngerer hoch qualifizierter Frauen (Interview BMF). Zweitens wird die Legitimität der Vereinbarkeitsfrage durch deren Ökonomisierung noch verstärkt: Ein

36 Aus der Internetseite der Initiative der Hertie-Stiftung: http://www.beruf-und-familie.de/ index.php?c=15 (Zugriff: 03.12.2015). 
individualisiertes Vereinbarkeitsangebot führe zu einer größeren Arbeitszufriedenheit und Motivation der MitarbeiterInnen und somit zum Anstieg ihrer Produktivität (Interview BMAS/BMF). Solche Kausalitäten entsprechen dem Geist der Modernisierung der Verwaltung und der Sprache des „,new public management" und setzen in der Vereinbarkeitspolitik willkommene, zeitgemäße Akzente - ganz im Gegensatz zum gerechtigkeitsorientierten Frauenförderungsdiskurs. Schließlich werden Breite und Vielfalt des Vereinbarkeitsangebots (weit jenseits der Teilzeitarbeit) zum wichtigsten „Wettbewerbsvorteil" des öffentlichen Dienstes gegenüber der Privatwirtschaft (Interview BMAS/BMF). Daher ist die Bundesverwaltung bemüht, dieses Angebot weiter zu verbessern, um die Folgen des demografischen Wandels und den kommenden Fachkräftemangel für den öffentlichen Dienst abzumildern (BMI 2010).

Zwischenfazit: Vereinbarkeit als Alleskönner der Gleichstellungspolitik

Maßnahmen zur Vereinbarkeit von Berufs- und Familienleben haben im Bereich beruflicher Gleichstellung einen zentralen Stellenwert erlangt. Abweichend von oder ergänzend $\mathrm{zu}$ den anderen Tendenzen lassen sich für beide Bundesministerien drei wesentliche Punkte identifizieren. Die Zentralität und hohe Akzeptanz des Vereinbarkeitsthemas in der Gleichstellungspolitik wird durch ein breites und heterogenes Bündel von Argumenten untermauert, das das Anliegen und die Problemlösung von einem breiten Konsens getragen erscheinen lässt. Die Vereinbarkeitspolitik nimmt rund um das zentralen Instrument der Teilzeitarbeit ausdifferenzierte Formen an, die den aktuellen Wünschen der Beschäftigten entsprechen: In Antizipation der zunehmenden Schwierigkeit der Rekrutierung von Fachkräften gewinnt die Flexibilisierung des Arbeitsortes auf den personalpolitischen Agenden an Bedeutung.

In diesem Sinn sind die Instrumente der Vereinbarkeitspolitik weit ausdifferenziert und individuell gestaltbar. Ihre Reichweite hat sich mit der Zeit erweitert, wie die Universalisierung des Teilzeitanspruchs deutlich macht: Während die Teilzeitregelungen ursprünglich für Frauen mit Kindern gedacht war, richten sie sich mittlerweile gleichermaßen an Männer wie an Frauen mit oder ohne Kinder. Bleibt das ungelöste Problem der Koordination mit der Personalpolitik. Während die Rechtsansprüche zumeist unter europäischem politischen Druck aufgrund vermuteter Diskriminierungsanfälligkeit starrer Arbeitszeitregelungen überprüft und überarbeitet wurden, bleibt der für Frauen diskriminierende Zusammenhang zwischen Teilzeitarbeit, Beurteilungsund Beförderungsmechanismen eine offene Baustelle. Damit sind Vereinbarkeitsmaßnahmen in ihren potenziellen gleichstellungspolitischen Auswirkungen weiterhin zwiespältig: Sie entsprechen den individuellen Bedarfen, bergen aber ein beträchtliches Diskriminierungspotenzial. 
Die Vereinbarkeitspolitik der Bundesverwaltung beinhaltet damit ein Paradox: Sie wird als Hauptstrategie der beruflichen Gleichstellung von Männern und Frauen dargestellt, ist in der Praxis jedoch eine Quelle für anerkannte Benachteiligungen der im Bundesdienst beschäftigten Frauen. Die Auswahl der Vereinbarkeitsinstrumente spiegelt nur bedingt die Reflexion der einzelnen Bundesministerien über diese Zwiespältigkeit bzw. einen eher unreflektierten Umgang mit diesen Instrumenten wider.

\subsubsection{Mehr Frauen in Führungsposition: Unzureichende Quotenregelungen}

Als zweites zentrales Problemfeld der Gleichstellungspolitik im Bundesdienst zeigt der Frauenanteil bei den Leitungsfunktionen eine stetige und positive Entwicklung über den Zeitraum der Berichterstattung. Von 19\% im Jahr 1999 ist der Anteil an Frauen in Leitungsfunktionen 2009 auf 30\% angestiegen, wenngleich er sich dann bis 2012 nicht mehr verändert hat (BMFSFJ/Kienbaum 2014a: 13). ${ }^{37}$ Im europäischen Vergleich befindet sich der deutsche Bundesdienst auf den untersten Rängen: In der zweithöchsten Position der Abteilungsleitung waren 2009 14\% Frauen in Deutschland vertreten, während es in Frankreich 25\% und in Schweden 44\% waren (Deutscher Bundestag 2010a: 19-24).

Die Abschaffung der Unterrepräsentanz von Frauen in Führungspositionen im Bundesdienst wird gesetzlich durch die sog. „leistungsabhängige Entscheidungsquote“, nämlich das Gebot der Bevorzugung von Frauen bei gleicher Qualifikation, geregelt. Das Problem wird aber auf Regierungsebene durch keine gezielteren Maßnahmen angegangen, obwohl das Thema im Koalitionsvertrag zwischen CDU, CSU und FDP 2009 als prioritär eingestuft wurde. Damals wurden in den einzelnen Bundesministerien Initiativen angekündigt, die das Problem schrittweise lösen sollten. Als Ursache der Ungleichheit werden vor allem ,bestimmte Arbeitskulturen und Arbeitsstrukturen wie etwa sehr lange Arbeitszeiten, häufige Dienstreisen oder ständige Verfügbarkeit" erwähnt, die auch in der Privatwirtschaft zu finden sind (Deutscher Bundestag 2010a: 27).

Entscheidungsquote: Konzept der „gleichen Qualifikation“

Das Gesetz sieht vor, dass offene Stellen mit dem Ziel der Erhöhung des Frauenanteils ausgeschrieben werden sollen, wenn in einem Bereich Frauen

37 Seit 2009 sind in den obersten Bundesbehörden und im mittelbaren Bundesdienst Zuwächse des Frauenanteils zu erkennen. Allerdings ist in der Gruppe der nachgeordneten Bundesbehörden ein Rückgang des Frauenanteils zu verzeichnen. Der Rückgang des Frauenanteils in den nachgeordneten Bundesbehörden gleicht den positiven Trend der obersten Bundesbehörden und des mittelbaren Bundesdienstes aus (ebd.). 
unterrepräsentiert sind, ${ }^{38}$ wenngleich die Ausschreibung in jedem Fall geschlechtsneutral formuliert sein muss ( $\$ 6$ BGleiG). Eine Teilzeitoption muss in jedem Fall auch bei Vorgesetzten- und Leitungsfunktionen ermöglicht werden. Die Ausschreibung soll dann öffentlich erfolgen, sofern nach einer internen Ausschreibung nicht genauso viele Bewerbungen von Frauen wie Männer vorliegen.

In den Bereichen, in denen Frauen unterrepräsentiert sind, sind zu den Vorstellungsgesprächen mindestens ebenso viele Frauen wie Männer einzuladen, sofern Bewerbungen von Frauen in entsprechender Zahl vorliegen $(\S 7$ BGleiG). Diese Quotierungsvorgabe gilt jedoch (anders als in manchen Bundesländern) nur in Fällen von deren Unterrepräsentanz. Auswahlkommissionen sollen geschlechterparitätisch besetzt sein; andernfalls sind die Gründe aktenkundig zu machen.

In Bereichen, in denen sie unterrepräsentiert sind, sind Frauen bei gleicher Eignung hinsichtlich Befähigung und Leistung bevorzugt zu berücksichtigen, ,sofern nicht in der Person eines Mitbewerbers liegende Gründe überwiegen“ ( $\S \S 8,9$ BGleiG). ${ }^{39}$ Diese 2001 eingeführte ,einzelfallbezogene Quote“ („Entscheidungsquote“) gilt bei Ausbildung, Besoldung, Einstellung, Anstellung und Beförderung. Entsprechend der europäischen Rechtsprechung $^{40}$ legt das BGleiG auch ein ausdrückliches Frageverbot zu privaten familienrelevanten Themen (geplante oder bestehende Schwangerschaft, Sicherung der Betreuung von Kindern oder der Pflege von Angehörigen) bei Bewerbungsgesprächen fest.

Die Einführung einer „Härtefallklausel“ führte auf der Bundesebene dazu, dass es eigentlich nur bei der Personalauswahl zu einem „harten“"Vergleich von gegebenen Qualifikationen zwischen den BewerberInnen kommt (Deutscher Bundestag 2010a: 34). Bei der Beförderung gilt nach wie vor die „Besten-Auslese-Strategie“ (ebd.), die dazu führt, dass im Auswahlprozedere individuellen und persönlichen Merkmalen der BewerberInnen der Vorrang gegeben wird, während der Aufgabenbezug des Arbeitsplatzes und die formale Qualifikation in den Hintergrund treten (ebd.). Bednarz-Braun (2000:

38 Frauen gelten als unterrepräsentiert, wenn ihr Anteil in einer Dienstelle in dem in Betracht gezogenen Bereich (Besoldungs-, Vergütungs- und Lohngruppen, Laufbahngruppen und Fachrichtungen sowie zusätzliche Funktionen mit Vorgesetzten- und Leitungsfunktionen) bei unter 50\% liegt. Die Bevorzugung sollte ab 2015 auch für Männer gelten, aber nur wenn deren Unterrepräsentanz in einem Bereich auf eine strukturelle Benachteiligung zurückzuführen ist. So lautet das novellierte BGleiG, das nach heftiger Kritik von ExpertInnen vom Bundestagausschuss für Familienpolitik geändert wurde. Der Gesetzentwurf sah ursprünglich keinen Bedarf vor, solche Benachteiligung zu beweisen.

39 Diese zusätzliche Formulierung wurde eingefügt, um den Vorzug von Frauen nicht zu automatisieren - was vom EuGH als diskriminierend bewertet wurde (siehe das „KalankeUrteil“" vom 17.101995; das „Marschall-Urteil“" von 11.11.1997, etc.; siehe dazu Schiek et al. 2002). Im Einzelfall soll auch die Einstellung von Männern möglich sein. Dies wird im EU-Jargon als „Härteklausel“ bezeichnet.

40 Siehe dazu das „Dekker-Urteil“ vom 08.11.90 und das „Mahlburg-Urteil“ vom 03.02.2000. 
139-142) betont diesbezüglich, dass ,eine dominante Meinung lautet [dass] es (...) gar keine Fälle gleicher Qualifikation geben“ könne. Verschiedene Studien und Berichte verweisen auf den Befund der systematischen Nichtanwendung sowohl der Entscheidungsquoten als auch der Ergebnisquoten: "Ausnahmen werden öfter in Anspruch genommen als das Regelverfahren“ (Schimeta 2012: 52). Zusätzliche Gründe für die Nichtanwendung der Entscheidungsquote seien deren Unverbindlichkeit und das Fehlen von Kontrolloder Sanktionsmechanismen (ebd.).

Ein Gutachten des ehemaligen Bundesverwaltungsgerichtsvorsitzenden Hans-Jürgen Papier für das Land Nordrhein-Westfalen rückte in Mai 2014 schließlich die Frage nach der Ausgestaltung der Entscheidungsquote ins öffentliche Licht: Entgegen der seit 2002 wiederholten Rechtsprechung des Bundesverwaltungsgerichts ${ }^{41}$ befürwortet das Gutachten eine gesetzliche Beschränkung der Ausdifferenzierung der Qualifikationsmerkmale, um die praktizierte Vermeidungs- oder Umgehungsstrategie außer Kraft zu setzen und die Anwendbarkeit der Quotenregelung wiederherzustellen. Obwohl das Gutachten eine wesentliche Rolle in der politischen Debatte und in der öffentlichen Anhörung des Bundestags um die Novellierung des BGleiG spielte, wurde eine eher noch weniger präzise Definition der Bewertung gleicher und ungleicher Qualifikation ${ }^{42}$ ins Gesetz geschrieben, was zu heftiger Kritik führte. ${ }^{43}$

Ergebnisquoten bei der Besetzung aller offenen Stellen und von Bundesgremien werden auch umgegangen

Das BGleiG enthielt in seinen Bestimmungen zum Gleichstellungsplan (§ 11) eine zweite Quotenart - die sog. „Ergebnisquote“ (Schiek et al. 2002: 122, 247), nach der ,mindestens die Hälfte der zu besetzenden Personalstellen eines Bereichs, in dem Frauen unterrepräsentiert sind, zur Besetzung durch Frauen vorzusehen" ist. Jedoch fehlte im Gesetz ein zeitlicher Rahmen für die Erfüllung dieses Ziels - eine gravierende Fehlkonstruktion für eine verbindliche Quote (Bothfeld/Rouault 2015), die dazu führte, dass diese Quote in der jüngsten Novellierung des BGLeiG verschwand. Als einziges wesentliches Novum enthält die 2015 durchgeführte Novellierung nun die Pflicht zur Festlegung von Zielgrößen für jede Leitungsebene und alle Teilbereiche der Verwaltung.

41 Das BVerwG bevorzugt nämlich seit seinem Urteil vom 19.12.2002 (BVerwG 2 C 1.02) eine weitere Ausdifferenzierung der Qualifikationsmerkmale im Falle gleicher Qualifikation.

42 Bisher bestimmte die Qualifikation sich ausschließlich nach den Anforderungen des konkret zu besetzenden Arbeitsplatzes. Zukünftig soll nach dem Gesetzentwurf die Qualifikation lediglich anhand der Anforderungen ermittelt werden.

43 Die schärfsten Kritiken kamen aus dem Deutschen Juristinnenbund (2015) und wurden in der Anhörung von der Vorsitzenden des IMA (Rose-Möhring 2015) geäußert. 
Die zweite eher implizite und unverbindliche „Ergebnisquote“ wurde durch das Bundesgremienbesetzungsgesetz 1994 eingeführt mit dem Ziel, mittelfristig eine paritätische Besetzung aller Bundesgremien zu erreichen. Die Parität sollte durch ein Doppelbenennungsverfahren ${ }^{44}$ erreicht werden. Diese Quote fand aber 15 Jahre später kaum mehr Anwendung. 2009 lag der Frauenanteil in den Bundesgremien im Durchschnitt bei 25\%, wobei sich der Anteil bis 2013 kaum geändert hatte und bei 25,7\% lag (BMFSFJ 2015b). Nur ein Ressort erreichte 2013 das gesetzliche Ziel der Parität in den von ihm beaufsichtigten Gremien, nämlich das BMFSFJ, während der Frauenanteil in den Gremien des BMF bei 22\% und des BMAS bei 31\% lag (Deutscher Bundestag 2010b: 14; 34). Eine positive aber langsame Entwicklung war anfangs zu beobachten, die sich später jedoch verlangsamte. Erst die Debatte ab 2011 um die Einführung einer gesetzlichen Quote für die Besetzung von Aufsichtsräten privater Unternehmen beschleunigte diese Entwicklung wieder und lie $\beta$ den Frauenanteil in den Bundesgremien ansteigen: Der Anteil an Gremien mit einem Frauenanteil von mindestens 30\% stieg ab 2010 um zehn Prozentpunkte und lag 2013 bei 41\% (BMFSFJ/Kienbaum 2014b: 1). Verantwortlich für die zähe Entwicklung in den Jahren davor waren unspezifische gesetzliche Ziele und Ernennungsentscheidungen, die dezentral und unkoordiniert getroffen wurden und als informell und intransparent gelten (Deutscher Bundestag 2010b: 31). Die Doppelbenennung wurde von der Regierung 2015 abgeschafft.

Demografische Entwicklung: Verlässliche Triebfeder für die Gleichstellung

Wie aufgrund früherer Analysen zu erwarten (Schimeta 2012; Bednarz-Braun 2000), kommen die einzelfallbezogenen Quoten in den zwei untersuchten Bundesministerien kaum zur Geltung. Im BMF verlässt man sich auf einen „Generationeneffekt", der langfristig den Frauenanteil in Führungspositionen automatisch erhöhen würde, gemäß dem Motto: „Die besseren Abschlüsse machen heutzutage sowieso die Frauen." 45 (Interview BMF) Erfahrene und qualifizierte Frauen befänden sich ,in der Warteschlange“ und würden auf die Leitungsfunktionen befördert, wenn sie an der Reihe seien (Interview BMF). Im BMAS stellt sich die Problematik der Rekrutierung und Beförderung völlig anders dar, da die meisten Bewerbungen ohnehin von Frauen kommen.

Von den GesprächspartnerInnen in beiden Bundesministerien wird der geringe Frauenanteil an Führungspositionen als momentan unlösbares Problem der Bundesverwaltung dargestellt und mit einem strukturellen Argument begründet: In Zeiten der Budgetkonsolidierung gebe es kaum Fluktuation auf

44 Für jede freie Gremienposition sollten (vor 2015) ein Kandidat und eine Kandidatin benannt oder vorgeschlagen werden.

45 Vor allem unter JuristInnen und BWLerInnen. 
dieser Leitungsebene, deren Posten von einer Generation von 55- bis 60jährigen Männern besetzt seien (Interview BMF).

Gleichstellungsbeauftragte und manche weibliche Vorgesetzte bevorzugen aber andere Praktiken, wenn Posten zu besetzen sind: Frauen werden informell, aber gezielt angesprochen und ermutigt, sich auf die offene Stelle $\mathrm{zu}$ bewerben. Zuweilen wird jedoch von potenziellen Kandidatinnen argumentiert, dass ein männlicher Kollege ,schon lange drauf wartet" (Interview BMAS), sodass sie sich nicht bewerben mögen, um nicht den Kollegen etwas wegzunehmen. Anstelle einer offiziellen Aufstiegsförderung der Frauen wird dann gleichsam ,im Schatten“ der Gleichstellungspolitik der Bundesministerien eine Strategie der individualisierten Förderung praktiziert.

\section{In der Zwischenzeit: Den Komplex Teilzeit - Beurteilung - Beförderung entflechten}

Wenn das Thema Lohngleichheit in den geführten Interviews angesprochen wurde, wurde in beiden Ministerien die Neutralität des Laufbahnrechts betont, die jede Geschlechterdiskriminierung bei der Entlohnung und der Beförderung verhindere. Allerdings belegt die einzige statistische Auswertung der Lohnstrukturen des öffentlichen Dienstes einen Gender Pay Gap von 8\% (BMFSFJ 2009). ${ }^{46}$ Auf Nachfrage wird jedoch die komplexe Interaktion zwischen Teilzeitarbeit, dienstlichen Beurteilungen, Notenkontingenten, Leistungsprämien und Beförderung skizziert und als diskriminierungsanfällig erkannt. Das Diskriminierungspotenzial wird aber nicht immer entsprechend in systematische Gegenmaßnahmen übersetzt. Allerdings unterscheiden sich hier die zwei Bundesministerien in ihren Praktiken.

Im BMAS wird vor allem über die Merkmale und Verfahren der dienstlichen Beurteilungen mit der Personalvertretung verhandelt. Zudem wurden sowohl neue Verfahren (Zweitbeurteilungen) und Kontrollindikatoren (Bewertungsspiegel) als auch Leitfäden und Schulungen für beurteilende Vorgesetzte eingeführt (vgl. Abschnitt 5.4.1). Diese Verhandlungen können sich über Jahre hinziehen - im BMFSFJ wurde etwa über vier Jahre lang verhandelt. Trotzdem würde die „Beurteilung aber immer noch subjektiv bleiben“ (Interview BMFSFJ). Auch besteht trotz Schulungen und wachsendem Bewusstsein für das Diskriminierungspotenzial der Beurteilungsverfahren das Problem der Benachteiligung von Teilzeitkräften fort. Die Existenz von Notenkontingenten erschwert eine geschlechtergerechte Bewertung zusätzlich. ${ }^{47}$

46 Allerdings liegt die Einkommensdifferenz bei den Beamtinnen bei nur 1,7\% und bei den Tarifbeschäftigten bei 7,8\%. Im Bundesdienst ist die Differenz mit nur 1\% gering im Vergleich zu $8 \%$ auf der Landesebene und 11\% auf der kommunalen Ebene (ebd.).

47 Das Prinzip der Notenkontingente sorgt dafür, dass nur eine bestimmte Zahl von Spitzennoten (,,erst- und zweitbeste Noten“) vergeben werden, die über dem Durchschnitt liegen. Die Kontingente beziehen sich jeweils auf Besoldungs-, Entgelt- oder Gehaltsgruppen in einem bestimmten Berichtszeitraum (Interview BMAS). 
Zwischenfazit: Unzureichend ausdifferenzierte Quotenregelungen

Die einzigen gesetzlich definierten Instrumente zur Beseitigung der Unterrepräsentanz von Frauen in Führungspositionen sind die Entscheidungsquote und die Ergebnisquote. Aussagen über ihre zum Teil systematische Nichtanwendung über die Zeit sind der Beweis für ihre Ineffektivität. Eine Ausnahme bildet hier die Phase des Beginns der Amtszeit der rot-grünen Regierung. Das Design beider Quoten erweist sich zudem als unzureichend. Ihre Reichweite wurde zwar breit angelegt, da sie bei Ausbildung, Besoldung, Einstellung, und Beförderung und Gremienbesetzung angewandt werden sollten. Allerdings sind die Quotenregelungen zu wenig an die spezifischen Kontexte angepasst: Es fehlen begleitende Maßnahmen, um ihre Umsetzung zu erleichtern. Hier bietet die norwegische Quotenregelung für die Privatwirtschaft ein interessantes Beispiel, da dort eine Datenbank für potenzielle Kandidatinnen für Aufsichtsratsposten eingerichtet wurden (Bothfeld/Rouault 2015). Zudem leidet die Quotenregelung an ihrer Fehlkonstruktion und büßt damit ein Mindestmaß an Verbindlichkeit ein: Zwar soll eine gleiche Präsenz auf allen Funktionsebenen erreicht werden, doch wurden für diesen Zweck keine Mindeststandards oder Zwischenziele und vor allem keine Fristen festgelegt, sodass jede Form von Erfolgskontrolle schwierig ist. Die Einführung von Zielvorgaben in die Gleichstellungspläne ab 2015 könnte sich als hilfreich erweisen, besonders wenn der gleichzeitig eingeführte Gleichstellungsindex der obersten Behörden deren Fortschritte regelmäßig misst und vergleicht.

\subsection{Fazit zur deutschen Bundesverwaltung: Ein normativ kohärentes, aber technisch inkonsistentes Steuerungsregime zur beruflichen Gleichstellung}

Nach Aussage unserer GesprächspartnerInnen in beiden untersuchten Ministerien sind für die gesamte Bundesverwaltung weder einheitliche Instrumentensets zur beruflichen Gleichstellung noch eine einheitliche gleichstellungspolitische Praxis zu erkennen: Ein erkennbarer politischer Impuls auf Regierungsebene - z.B. in Form eines Gleichstellungsprogramms für die gesamte Bundesverwaltung - fehlt seit Jahren und die jüngste Novellierung des BGleiG hat sich als eher enttäuschend entpuppt, da sie keine systematische Verbesserung der Instrumente und sogar einige Verschlechterungen beinhaltet (vgl. u.a. Deutscher Juristinnenbund 2014). Stattdessen gibt es einige Themen, die einen „kleinsten gemeinsamen Nenner“ im Bereich beruflicher Gleichstellung in allen Bundesministerien bilden. Dazu gehört einerseits die Vereinbarkeit von Beruf und Familie und anderseits die Förderung von Frauen in Führungspositionen. Trotz dieser Unzulänglichkeiten verfügt der Bun- 
desdienst jedoch über stark institutionalisierte gleichstellungspolitische Instrumente: Die Gleichstellungsbeauftragten und das Instrument der Gleichstellungspläne stellen seit über 30 Jahren die Pfeiler einer institutionellen Gleichstellungspolitik dar, die sich inkrementell in der alltäglichen Verwaltungspraxis verwurzelt haben.

Die Instrumente zur Herstellung der beruflichen Gleichstellung in der Bundesverwaltung sind in ihrer gesetzlichen Ausgestaltung mit einer relativ hohen Verbindlichkeit ausgestattet und stellen im internationalen Vergleich einen imposanten Pflichtenkatalog dar - mit einer wichtigen Ausnahme: Das zentrale Instrument des Gleichstellungsplans, das zur Integration gleichstellungspolitisch relevanter Merkmale in der strategischen Planung der Personalpolitik beitragen sollte, bleibt trotz wiederholter Kritik und Reformversuche faktisch unverbindlich.

Nicht alle gleichstellungspolitischen Instrumente sind gleichermaßen ausdifferenziert. Während die Rechte der Gleichstellungsbeauftragten und die Instrumente zur Vereinbarkeit von Familie und Berufsleben gesetzlich weit aufgefächert sind und sich für sehr unterschiedliche Fälle gut anwendbar zeigen, sind die Maßnahmen zur Aufstiegsförderung von Frauen und die Regeln zur Erstellung der Gleichstellungspläne eher unterentwickelt, was die Implementationsdefizite im Wesentlichen erklärt. Die Reichweite der einzelnen Instrumente erstreckt sich hingegen stets auf die Gesamtheit der Beschäftigten der jeweiligen Bundesministerien und ihrer nachgeordneten Behörden. In Tafel 5.2 sind die einzelnen Instrumente zur Herstellung beruflicher Gleichstellung in der Bundesverwaltung vergleichend zusammengefasst, und sie illustriert die gesetzliche Ausgestaltung eines Steuerungsregimes, das insgesamt nur mäßig verbindlich, hingegen aber teilweise recht ausdifferenziert und in den meisten Bereichen recht weitreichend ausgestaltet ist. Insgesamt jedoch bietet es nur mäßig gute Voraussetzungen für eine effektive Integration gleichstellungspolitischer Instrumente mit der Personalpolitik der obersten Bundesbehörden.

Die produktive Verknüpfung von Gleichstellungspolitik und Personalpolitik, d.h. die Einbeziehung gleichstellungspolitischer Instrumente (Gleichstellungspläne) oder Akteure (Gleichstellungsbeauftragte) in die alltäglichen Routinen der Personalpolitik, erfolgt in den zwei beobachteten Bundesministerien tatsächlich nur bedingt. Im BMAS werden zwar wiederholt einzelne Initiativen ergriffen, im BMF verbleiben die Aktivitäten oft bei der Abarbeitung der gesetzlichen Anforderungen - was individuelle Initiativen aber nicht verhindert - z.B. das gezielte Coaching potenzieller Kandidatinnen zur Beförderung. Die Verknüpfung von beidem, Personal- und Gleichstellungspolitik, hängt schließlich auch stark von der personellen Besetzung der Führungsebene $\mathrm{ab}$ (Geschlecht, Alter, Einstellungen der Führungskräfte) insbesondere in der Personalverwaltung. Auch die Legitimität und Einbeziehung der Gleichstellungsbeauftragten in strategische personalpolitische Entschei- 
Tafel 5.2: Vergleichende Bewertung der Instrumente zur beruflichen Gleichstellung in der deutschen Bundesverwaltung

\begin{tabular}{|c|c|c|c|c|}
\hline $\begin{array}{l}\text { Gleichstel- } \\
\text { lungspolitische } \\
\text { Instrumente/ } \\
\text { Bewertung (mit } \\
\text { entsprechen- } \\
\text { den Merkmalen) }\end{array}$ & Verbindlichkeit & $\begin{array}{l}\text { Ausdifferen- } \\
\text { ziertheit }\end{array}$ & Reichweite & $\begin{array}{l}\text { Voraussetzun- } \\
\text { gen zur Integra- } \\
\text { tion in der } \\
\text { Personalpolitik } \\
\text { des Ressorts/ } \\
\text { Bewertung }\end{array}$ \\
\hline $\begin{array}{l}\text { Gleichstel- } \\
\text { lungsbeauftrag- } \\
\text { te }\end{array}$ & $\begin{array}{l}\text { Stark } \\
\text { Bestellung per } \\
\text { Wahl (++); Mit- } \\
\text { wirkung an indi- } \\
\text { viduellen (++)/ } \\
\text { strategischen (-) } \\
\text { personalpoliti- } \\
\text { schen Entschei- } \\
\text { dungen } \\
\text { Klagemöglichkeit } \\
(+)\end{array}$ & $\begin{array}{l}\text { Mittel } \\
\text { Interne Vernet- } \\
\text { zung (++); } \\
\text { Anerkennung der } \\
\text { fachlichen } \\
\text { Kompetenzen (-) }\end{array}$ & $\begin{array}{l}\text { Sehr stark } \\
1 \text { Diskriminie- } \\
\text { rungsmerkmal } \\
(+) \text {; Weisungs- } \\
\text { freiheit (++); } \\
\text { Zuordnung zu } \\
\text { Leitung der } \\
\text { Dienststelle (+); } \\
\text { Zeit-/Personal- } \\
\text { budget nach } \\
\text { Mitarbeiterzahl } \\
(++)\end{array}$ & Relativ stark \\
\hline $\begin{array}{l}\text { Gleichstel- } \\
\text { lungspläne }\end{array}$ & $\begin{array}{l}\text { Schwach } \\
\text { Keine externe } \\
\text { Veröffentlichung } \\
\text { (--); keine Sank- } \\
\text { tion (--); Klage- } \\
\text { recht der GBA } \\
(+)\end{array}$ & $\begin{array}{l}\text { Relativ schwach } \\
\text { Turnus/Aktuali- } \\
\text { sierung (+); } \\
\text { wenige Anwei- } \\
\text { sungen (-); Tiefe/ } \\
\text { Umfang (+/-) }\end{array}$ & $\begin{array}{l}\text { Schwach } \\
\text { Kein standardi- } \\
\text { sierter Vergleich } \\
(-)\end{array}$ & Schwach \\
\hline $\begin{array}{l}\text { Vereinbar- } \\
\text { keitsmaßnah- } \\
\text { men }\end{array}$ & $\begin{array}{l}\text { Stark } \\
\text { Gesetzliche } \\
\text { Verankerung } \\
(++) \text {; begleitende } \\
\text { Maßnahmen } \\
\text { (Gleichstellungs- } \\
\text { plan: +); politi- } \\
\text { scher Impuls } \\
\text { (Zertifizierung: +) }\end{array}$ & $\begin{array}{l}\text { Sehr stark } \\
\text { Bandbreite von } \\
\text { Arbeitszeitarran- } \\
\text { gements (++); } \\
\text { Urlaubsregelung }\end{array}$ & $\begin{array}{l}\text { Mittel } \\
\text { An Männer und } \\
\text { Frauen gerichtet } \\
(+) ; \\
\text { aber bisher gro- } \\
\text { ße Geschlech- } \\
\text { terspezifizität } \\
\text { (Teilzeit, Eltern- } \\
\text { geld) (-) }\end{array}$ & Stark \\
\hline $\begin{array}{l}\text { Aufstiegsförde- } \\
\text { rung }\end{array}$ & $\begin{array}{l}\text { Relativ stark } \\
\text { Gesetzliche } \\
\text { Verankerung } \\
(++) ; \text { gesetzliche } \\
\text { Quote (+); neue } \\
\text { begleitende } \\
\text { Maßnahme } \\
\text { (Zielvorgabe, } \\
\text { Index: +) } \\
\text { Diskriminie- } \\
\text { rungsverbot (+) }\end{array}$ & $\begin{array}{l}\text { Mittel } \\
\text { Prozessregelun- } \\
\text { gen von Perso- } \\
\text { nalverfahren (+); } \\
\text { gezielte individu- } \\
\text { elle Förderung } \\
(-)\end{array}$ & $\begin{array}{l}\text { Mittel } \\
\text { Von Rekrutie- } \\
\text { rung bis Beförde- } \\
\text { rung (+); } \\
\text { neue Inklusion } \\
\text { von Männerför- } \\
\text { derung (-) }\end{array}$ & Mittel \\
\hline Steuerungsreg. & Mittel & Mittel & Mittel & Mittel \\
\hline
\end{tabular}

Quelle: eigene Zusammenstellung 
dungen sind entscheidende Momente, die sich in der Praxis jedoch selten finden lassen.

Das Steuerungsregime zur beruflichen Gleichstellung im deutschen Bundesdienst zeichnet sich somit vor allem durch den Mangel eines starken Monitorings aus. Dort, wo keine materiellen Sanktionen möglich sind, würde allein das Sichtbarmachen der Nichterfüllung gesetzlicher Vorgaben durch Prüfung und Vergleich einen Handlungsdruck für die Bundesministerien entstehen lassen. Die Autonomie der Bundesministerien enthebt diese jedoch der Rechenschaftspflicht gegenüber dem BMFSFJ. Die nach innen gerichtete und von der Verwaltungshierarchie abhängige Funktion der GBA kann nur bedingt eine kompensierende Kontrollfunktion ausüben, sodass das Steuerungspotenzial der im Gesetz vorgesehenen Instrumente nicht ausgeschöpft werden kann.

Die Politik zur beruflichen Gleichstellung im Bundesdienst ist somit durch ein normativ relativ kohärentes, aber zum Teil technisch inkonsistentes Steuerungsregime gekennzeichnet. Dieses Steuerungsregime bleibt wiederum in einem inkonsistenten (weil nur begrenzt modernisierten) und inkohärenten (weil völlig normativ gespaltenen) Gleichstellungsregime eingebettet.

Inwiefern zeichnet sich mit der Novellierung des BGleiG nun ein Kurswechsel unter der zweiten Großen Koalition ab? Abwarten ist hier angebracht. Das novellierte BGleiG soll zunächst seinen Geltungsbereich erweitern, indem es erstmals auch für jene Unternehmen gilt, an denen der Bund mehrheitlich beteiligt ist. Außerdem soll der Gleichstellungsplan zukünftig eine quantifizierte Zielvorgabe für jede einzelne Vorgesetzten- und Führungsebene enthalten. Gekoppelt an die Veröffentlichung eines jährlichen Gleichstellungsindex der obersten Bundesbehörden, werden Bundesministerien möglicherweise erstmals zum Handeln gezwungen, unter der Androhung, sich andernfalls als widerständige Arbeitgeber öffentlich zu blamieren. Die Novellierung des BGleiG scheint auf etwas mehr Vergleichbarkeit von ministerialen gleichstellungspolitischen Praktiken zu setzen (BMFSFJ 2014): Ein erhöhter Druck wird dadurch auf die Bundesverwaltung ausgeübt, sodass sie ihre Vorbildfunktion weiterentwickelt - umso mehr, als das im März abgeschlossene „Frauenquotengesetz“ den Druck auf private Unternehmen wesentlich erhöht und den Wettbewerbseifer zwischen privaten und öffentlichen Arbeitgebern schürt. 


\section{$6 \quad$ Gleichstellungspolitik im österreichischen Bundesdienst}

\section{Andrea Leitner}

Betriebliche Gleichstellungspolitik nimmt eine Schlüsselrolle beim Abbau struktureller Benachteiligungen von Frauen im Erwerbsleben ein. Die Einführung und Umsetzung von Maßnahmen in privatwirtschaftlichen Betrieben wird aber in Österreich als „quantitativ zurückhaltend“ und „qualitativ oft mangelhaft" eingeschätzt (Auer/Welte 2009: 193). Dementsprechend kommt dem öffentlichen Dienst eine wichtige Vorbildfunktion für die Etablierung einer effektiven Gleichstellungspolitik zu. Als Erwerbsorganisation und Arbeitgeber verfügt der Bundesdienst über einen großen Gestaltungsspielraum mit direktem Durchgriffsrecht der PolitikerInnen für gleichstellungspolitische Interventionen und ist dabei in doppelter Weise von der politischen Kultur beeinflusst: Zum einen durch die Mitwirkung von PolitikerInnen, zum anderen ist er, wie alle anderen Betriebe und Sektoren auch, in die allgemeine Gleichstellungspolitik eingebunden. Forschungsleitend für die Analyse der österreichischen Fallstudie war, wie weit sich die Gleichstellungspolitik im öffentlichen Dienst von jener in der Privatwirtschaft unterscheidet. Nimmt die Gleichstellungspolitik des Bundesdienstes eine Funktion als „Erzieher des Volkes“" (Göhler 1997) ein, indem diese stärker an egalitätsorientierten Ansätzen zur Gleichstellung von Frauen und Männern orientiert ist als die auf Vereinbarkeitspolitik konzentrierte, differenzorientierte Gleichstellungspolitik Österreichs?

Die Expertise folgt im Aufbau den Fragestellungen des Ländervergleichs zwischen der Schweiz, Deutschland und Österreich. Für die Umsetzung war aber der unterschiedliche Forschungs- und Informationsstand zur Gleichstellungspolitik im Bundesdienst mit entscheidend: In Österreich werden Planungsdokumente und Monitoringberichte zur Umsetzung von Gleichstellung in den Ministerien regelmäßig publiziert. Hingegen besteht wenig wissenschaftlicher Diskurs über Gleichstellungspolitik im öffentlichen Sektor, abgesehen von den nunmehr ausgelagerten Institutionen des Arbeitsmarktservice und der Hochschulen. Auer et al. haben bereits im Jahr 1977 ein Vakuum hinsichtlich der Problemwahrnehmung des Beschäftigungsfeldes Frauen im öffentlichen Dienst festgestellt. Mit der Evaluierung des Förderprogramms für Frauen im Bundesdienst (Kreisky/Walther 1990) wurde eine umfassende Bestandsaufnahme über Gleichstellung geschaffen, aber eine aktuelle Analyse der Gleichstellungspolitik im Bundesdienst fehlt und kann im Rahmen dieser Expertise nur begrenzt geboten werden. Die vorliegende Analyse ist auf Wirkungsvoraussetzungen des Steuerungsregimes konzentriert, d.h. auf die Ausgestaltung von Regeln, Institutionen und Maßnahmen sowie ihre 
Kohärenz und Konsistenz im Kontext des Gender- und Policy-Regimes, während die Umsetzung, die soziale Praxis der Gleichstellungspolitik, im Rahmen dieser Expertise offen bleiben muss.

Die Effektivität dieses Steuerungsregimes wird für Österreich - wie in den beiden anderen Ländern - vor allem durch Aufarbeitung verfügbarer Daten zur Beschäftigungssituation im Bundesdienst, öffentlich zugänglicher Dokumente der Ministerien (wie Frauenförderpläne und Gleichbehandlungsberichte) analysiert und dabei auf zentrale Aspekte aus älteren Analysen Bezug genommen (vor allem Kreisky/Walther 1990). Zum Verständnis der vorliegenden Dokumente und Daten wurden im Sommer 2013 Gespräche mit VertreterInnen des Sozialministeriums, des Finanzministeriums sowie des Bundeskanzleramtes, die den Vorsitz der interministeriellen Arbeitsgruppe für Gleichstellungspolitik ausüben, durchgeführt. ${ }^{1}$

\subsection{Kontextbedingungen im Spannungsfeld von Familien- und Fraueninteressen}

Die innerpolitische Diskussion der Gleichstellungspolitik ist in Österreich durch die Konfliktlinie „Fraueninteressen versus Familieninteressen“ geprägt, was dazu führt, dass Fraueninteressen teilweise mit Familieninteressen gleichgesetzt werden (Appelt 2009: 31). Damit wirkt die Tradition eines differenzorientierten Wohlfahrtssystems, das eine klare Geschlechtertrennung der Erwerbs- und Versorgungsökonomie vorsieht, trotz punktueller gleichheitsorientierter Reformen im Ehe- und Familienrecht und im System der sozialen Sicherheit weiter (Dackweiler 2005). Das damit verfolgte Geschlechterregime wurde durch die Frauen- und Gleichstellungspolitik nicht aufgehoben, sondern hat zu einem Policy-Mix beigetragen, der durch Ambivalenzen und Inkonsistenzen geprägt ist (Wroblewski/Leitner 2005). Politische Strategien zur erwerbsarbeitsvermittelten Individualisierung von Frauen gehen mit einer familienorientierten Unterhaltslogik im Steuer- und Sozialrecht einher (Mairhuber/Sardadvar 2012). Besonders deutlich werden die widersprüchlichen Logiken des Geschlechterregimes im Zusammentreffen politischer Maßnahmen zur diskriminierungsfreien Arbeitsmarktintegration mit der konservativen Familienpolitik, die weiterhin eine traditionelle Arbeitsteilung der Geschlechter durch die finanzielle Unterstützung langer Ka-

1 Ich möchte mich an dieser Stelle sehr herzlich bei den GesprächspartnerInnen bedanken, die ihr Wissen und ihre Einschätzungen über Gleichstellungspolitik im Bundesdienst zur Verfügung gestellt und erste Hypothesen diskutiert haben. Darüber hinaus möchte ich mich auch bei Anna Dibiasi, die viele Daten und Informationen zum öffentlichen Dienst zusammengetragen hat, und Viktoria Englmair, die in der Endphase zur Fertigstellung des Buchprojekts beigetragen hat, bedanken. 
renzierungen von Müttern und unzureichende Kinderbetreuungsangebote unterstützt. Dies wirkt sich nicht nur auf die Kosten des Sozialsystems aus, indem beschäftigungspolitische Maßnahmen notwendig sind, um nicht intendierte arbeitsmarktpolitische Effekte der Familienpolitik zu korrigieren, sondern zeigt sich auch an der begrenzten Arbeitsmarktintegration von Frauen.

Entsprechend den Zielsetzungen der EU ist es in Österreich gelungen, den Anteil der erwerbstätigen Frauen wesentlich zu erhöhen: Die Erwerbsquote der Frauen ist in Österreich von 62\% (1995) über 64\% (2002) auf 71\% im Jahr 2013 gestiegen, während jene der Männer im Vergleichszeitraum annähernd gleich geblieben ist (1995 und 2013: 81\%). Doch dabei handelt es sich um eine begrenzte Integration, denn Frauenerwerbstätigkeit ist angesichts der Doppelrolle der Frauen in Beruf und Familie weiterhin durch lange Berufsunterbrechungen und in zunehmendem Maße durch Teilzeitbeschäftigung charakterisiert. Der Anstieg der Frauenerwerbstätigkeit ist vor allem auf den Zuwachs von Teilzeitbeschäftigten zurückzuführen (Abb. 6.1).

Abb. 6.1: Erwerbsquote und Teilzeitquote (1995-2013, in \%)

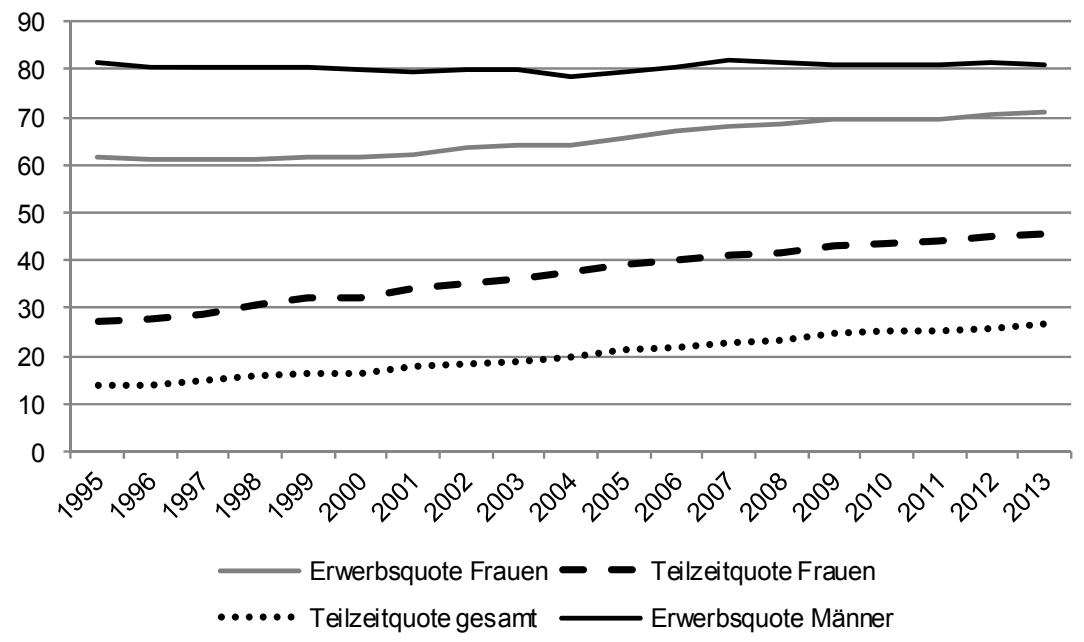

Quelle: eigene Darstellung auf Grundlage der Daten von Statistik Austria: bis 2003: Mikrozensus, 2004 bis 2013: Mikrozensus-Arbeitskräfteerhebung

Die Teilzeitquote der Frauen stieg in diesem Zeitraum von 27\% auf $46 \%$ (Männer 1995: 4\%, 2013 10\%). Teilzeitbeschäftigung betrifft insbesondere Frauen mit Kindern: $71 \%$ der Frauen im Alter von 25 bis 49 Jahren und mit Kindern unter 15 Jahren waren im Jahr 2012 teilzeitbeschäftigt (Statistik Austria: Mikrozensus-Arbeitsmarkterhebung 2012). Wird bei der Erwerbsbeteiligung das Beschäftigungsausmaß berücksichtigt, d.h. diese in Vollbeschäftigungsäquivalenten gemessen, so verringert sich die Erwerbsquote der 
Frauen auf 41\% (2013). Werden die Erwerbspersonen, um jene Frauen korrigiert, die sich in Elternkarenz befinden, sinkt die Erwerbsquote um weitere 2 Prozentpunkte (70.200 der erwerbstätigen Frauen sind in Elternkarenz; Statistik Austria: Abgestimmte Erwerbsstatistik 2012).

Wie die Evaluationen der Karenzregelungsreformen (2002, 2008, 2010) und die Einführung des Anspruchs auf Elternteilzeit (2004) gezeigt haben, werden die Erwerbsmuster von Frauen wesentlich durch wohlfahrtsstaatliche Leistungen und Regelungen zur Vereinbarkeit von Familie und Beruf bestimmt (Lutz 2004; Riesenfelder et al. 2006; Dörfler et al. 2009; RillePfeiffer/Kapella 2012). Mit 16 Wochen Mutterschutz (bei vollem Einkommensausgleich), der Möglichkeit der Elternkarenz bis zum zweiten Geburtstag des Kindes, dem Bezug von Kinderbetreuungsgeld bei fehlender bzw. eingeschränkter Erwerbstätigkeit von bis zu 30 Monaten $^{2}$ sowie dem Anspruch auf Elternteilzeit bis zum siebenten Geburtstag des jüngsten Kindes weist Österreich Anreize zu langen Erwerbsunterbrechungen und Teilzeitbeschäftigung von Frauen auf. 46\% der Österreicherinnen unterbrechen ihre Erwerbstätigkeit nach der Geburt eines Kindes für mehr als fünf Jahre. Bei Frauen, die bereits vor der Geburt längere Beschäftigungslücken aufweisen, erhöht sich dieser Anteil auf 59\% (Riesenfelder 2013). ${ }^{3}$ Trotz Reservierung eines Sechstels der Zeit für den zweiten Elternteil sind nur 7\% der KarenzgeldbezieherInnen Männer; d.h. Männer beteiligen sich nach wie vor nur in einem geringen Ausmaß an der Elternkarenz, was auch mit der geringen finanziellen Unterstützung zusammenhängt (Reidl et al. 2013).

Der vergeschlechtlichte Wohlfahrtsstaat setzt auf die familiäre Rolle von Frauen und trägt somit dazu bei, die traditionelle geschlechtsspezifische Arbeitsteilung und die damit verbundene Geschlechterhierarchie zu zementieren (Appelt 2009). Angesichts der gegebenen Kinderbetreuungsmöglichkeiten von Vorschul-, aber auch von Schulkindern ist eine Vollzeitbeschäftigung von Eltern - primär wieder Müttern - kaum möglich. Der Ausbau von Kinderbetreuungseinrichtungen wurde in den letzten Jahren forciert. Damit ist der Anteil der in Kindertagesheimen betreuten Kinder bei den bis Zweijähri-

2 Beim Kinderbetreuungsgeld kann zwischen fünf Varianten gewählt werden, die sich nach Bezugsdauer und Unterstützungshöhe unterscheiden, aber entsprechend der gesamten Geldleistung längere Varianten unterstützen. Das Kinderbetreuungsgeld kann als Pauschalleistung (vier Varianten: $30+6$ Monate mit 14,53 Euro Taggeld, $20+4$ Monate mit 20,80 Euro Taggeld, $15+3$ Monate mit 26,60 Euro Taggeld, $12+2$ Monate mit 33,00 Euro Taggeld) oder als einkommensabhängige Variante $(12+2$ Monate mit $80 \%$ des Letzteinkommens bzw. maximal 66,00 Euro Taggeld) bezogen werden. Mit Ausnahme des einkommensabhängigen Kinderbetreuungsgeldes ist der Bezug unabhängig von einer vorhergehenden Erwerbstätigkeit der Eltern sowie von arbeitsrechtlichen Karenzregelungen. Zudem existiert eine Zuverdienstgrenze, die eine Teilzeitbeschäftigung begünstigt (Arbeiterkammer: http://www.arbeiterkammer.at/beratung/berufundfamilie/kinderbetreungsgeld/Kinder betreuungsgeld-Varianten.html; Zugriff: 13.02.2015).

3 Diese Analyse erfolgte auf Basis von Administrativdaten für alle Frauen in Österreich, die im Jahr 2006 eine Geburt aufwiesen. 
gen zwischen 9\% im Jahr 2002 auf 21\% im Jahr 2012 gestiegen; bei den Drei- bis Fünfjährigen von $81 \%$ auf $91 \%$. Das Angebot ist allerdings regional sehr unterschiedlich, vor allem bei Kinderbetreuungseinrichtungen mit Öffnungszeiten, die eine Vollzeitbeschäftigung erlauben. Mehr als ein Viertel der Kindergärten bietet keine Nachmittagsbetreuung an, oft kein Mittagessen, und mit durchschnittlich 35 Schließtagen sind die Ferienzeiten häufig deutlich länger als die Urlaubszeiten von Erwerbstätigen (Statistik Austria 2014). Mangelnde Nachmittagsbetreuungsmöglichkeiten und lange Ferienzeiten charakterisieren auch die Betreuungssituation von Schulkindern.

Beschäftigungspolitische Maßnahmen für Frauen durch Qualifizierungen und Beratungen adressieren in einem hohen Ausmaß Wiedereinsteigerinnen, d.h. Frauen mit längerfristigen Berufsunterbrechungen wegen Kinderbetreuung: Im Jahr 2012 wurde knapp ein Fünftel der für Frauen verausgabten Fördermittel der aktiven Arbeitsmarktpolitik für Wiedereinsteigerinnen verwendet (Lutz et al. 2013). Dies ist angesichts der mit langen Berufsunterbrechungen verbundenen Wiedereinstiegsrisiken auch notwendig, weist aber auf die Ambivalenz der Politik hin. Die Vereinbarkeit von Familie und Beruf führt unter den gegebenen Rahmenbedingungen fehlender Kinderbetreuungsmöglichkeiten und verlängerten Karenzzeiten zu langen Berufsunterbrechungen, die dem Ziel der Beschäftigungspolitik, der Erhöhung der Erwerbsbeteiligung, entgegenstehen.

Mit der Verankerung von Gender Mainstreaming und Gender Budgeting, mit punktuellen Maßnahmen zum Abbau der beruflichen Segregation (z.B. Förderung von Mädchen in technischen Ausbildungen) sowie Ansätzen zur Erhöhung des Frauenanteils in Führungspositionen und zur Verringerung von Einkommensunterschieden bestehen Versuche, auch die vertikale und horizontale Segregation am Arbeitsmarkt zu mindern. Doch dabei zeigen sich kaum Fortschritte. Die berufliche Segregation hat sich über die letzten Jahrzehnte nur wenig verringert, die Konzentration von Frauen in pädagogischen und Pflegeberufen ist eher noch gestiegen (Leitner/Dibiasi 2015). ${ }^{4}$ Auch bei der vertikalen Segregation zeigt Österreich einen großen Nachholbedarf. Beim Anteil der Frauen in Führungspositionen liegt Österreich mit 30\% im Jahr 2013 ebenfalls deutlich hinter den nordeuropäischen Ländern und unter dem EU-Schnitt (European Commission 2014).

Die großen Geschlechterunterschiede in der beruflichen Positionierung gipfeln in hohen Einkommensunterschieden. Österreich weist im EU-

4 Der Dissimilaritätsindex ist - wie auch andere Segregationsmaßzahlen - in Österreich zwischen 1995 und 2012 nur wenig gesunken: von 0,56 auf 0,54. Das heißt, im Jahr 2012 mussten 54\% der beschäftigten Frauen und Männer ihren Beruf wechseln, um eine berufliche Gleichverteilung der Geschlechter zu erreichen. Die Veränderung geht mit einem steigenden Beschäftigtenanteil in integrierten Berufen, d.h. geschlechtsspezifisch ausgeglichenen Berufen einher, während aber die Dominanz von Frauen in pädagogischen und Pflegeberufen steigt. Beispielsweise ist der Frauenanteil bei Vorschul- und Primarlehrkräften von 88\% im Jahr 1998 auf 96\% im Jahr 2012 gestiegen. 
Vergleich mit 23\% den zweithöchsten Gender Pay Gap auf (ebd.), er ist gegenüber 26\% im Jahr 2012 nur wenig gesunken. Einen wesentlichen Einfluss hat neben der begrenzten Integration von Frauen durch Berufsunterbrechungen und Teilzeitbeschäftigung die branchen- und berufsspezifische Segregation des Arbeitsmarktes. Die Geschlechtersegregation bestimmt in einem deutlich stärkeren Ausmaß als in den nordeuropäischen Ländern - die ebenfalls eine hohe Segregation aufweisen - die Einkommensunterschiede zwischen Frauen und Männern mit (Biffl 2010). Ein Viertel des Gender Pay Gap wird durch unterschiedliche Branchen und Berufe erklärt (Geisberger/Glaser 2014). Maßnahmen zur Erhöhung der Einkommenstransparenz, wie die verpflichtende Angabe von Mindesteinkommen in Stelleninseraten und die Einkommensberichtspflicht für größere Unternehmen, zeigen bislang noch wenig Wirkung auf den Pay Gap.

\subsection{Frauen im österreichischen Bundesdienst}

Die Bundesverwaltung in Österreich stellt nicht nur aufgrund der direkten Durchgriffsmöglichkeiten der Politik für betriebliche Gleichstellung ein interessantes Beschäftigungssegment dar, sondern ist zugleich einer der größten Arbeitgeber des Landes. Im Jahr 2013 sind 4\% aller Beschäftigten dem Bundesdienst zuzurechnen - wenngleich der Personalstand des Bundes im Zeitraum von 1990 bis 2013 durch Ausgliederung von Trägerorganisationen und Stelleneinsparungen beinahe halbiert wurde. ${ }^{5}$

Von den rund 139.000 Bundesbediensteten sind im Jahr 2013 41\% Frauen. Die Feminisierung des Bundesdienstes fand vor allem in den 1980er Jahren statt, als der Frauenanteil zwischen 1980 und 1988 von 23\% auf 37\% stieg. Nach Kreisky und Walther (1990) ist diese Feminisierung wesentlich auf die innovative Schubkraft des Förderungsprogramms für Frauen im Bundesdienst zurückzuführen, das im Jahr 1981 implementiert wurde. Wären die durchschnittlichen Wachstumswerte aus den Jahren 1980 bis 1988 fortgeschrieben worden, hätte der Frauenanteil schon 1995 die 50\%-Marke überschritten. Der Frauenanteil ist danach aber in einem deutlich geringeren Ausmaß gestiegen, und es würde nach den aktuellen Wachstumsraten noch weitere 40 Jahre dauern, um eine Frauenquote von 50\% zu erreichen.

Verglichen mit der Privatwirtschaft ist der Anteil der Teilzeitbeschäftigten im Bundesdienst mit 17\% relativ gering (gegenüber $27 \%$ in der Gesamtwirtschaft). Das Charakteristikum von Teilzeitbeschäftigung als überwiegend weibliche Beschäftigungsform gilt aber auch für den Bundesdienst. Der Anteil der teilzeitbeschäftigten Frauen ist von knapp 20\% 1995 auf 33\% im Jahr

5 Laut Stellenplan 2012 sank der Personalstand des Bundes von 306.000 Beschäftigten im Jahr 1990 auf 161.000 Beschäftigte 2013. 
2013 gestiegen (vgl. Abb. 6.2). Dieser Anstieg verläuft damit sichtbar langsamer als jener für Gesamtösterreich. Bei Männern ist der Anteil der Teilzeitbeschäftigten hingegen kaum gestiegen. Diese Entwicklungen weisen darauf hin, dass im Bundesdienst Vollzeitarbeit stärker die Norm bleibt als in anderen Sektoren.

Abb. 6.2: Entwicklung des Frauenanteils und der Teilzeitquote im Bundesdienst (1995 bis 2013)

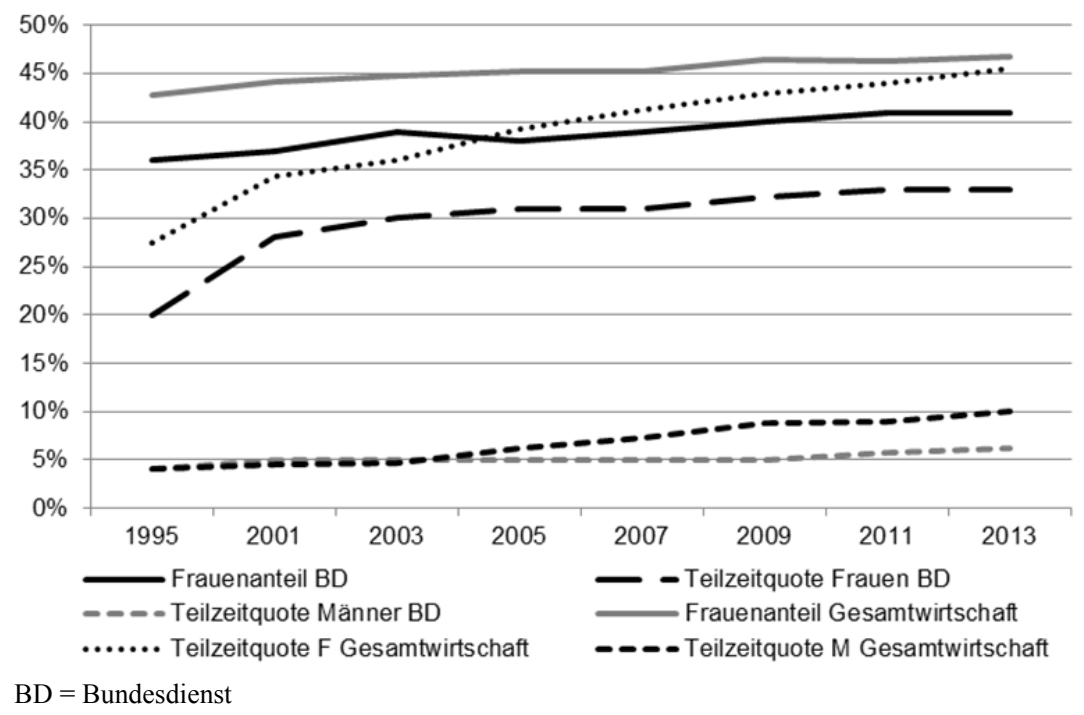

Quelle: eigene Darstellung auf Grundlage folgender Daten: Frauenanteil der Beschäftigten: 2001-2013: Gleichbehandlungsberichte (2002 bis 2014); 1995: BKA (2013a). Teilzeitquote: 1995-2005: BKA (2013a); 2007-2013: BKA Gleichbehandlungsberichte (2010 bis 2014)

Um genauere Aussagen über die Feminisierung des Bundesdienstes treffen zu können, soll im Folgenden seine Struktur näher erläutert werden.

\subsubsection{Feminisierung mit Ausnahme von Militär- und Exekutivdienst}

Wie schon in Kapitel 3 in diesem Buch erwähnt, ist der Anteil der Bundesbediensteten innerhalb des öffentlichen Sektors deutlich größer als in den beiden Vergleichsländern. In Österreich sind $38 \%$ aller Beschäftigten des öffentlichen Dienstes Bundesbedienstete, 40\% Landesbedienstete und 22\% Gemeindebedienstete (BKA 2013a). Dies hängt mit der Aufgabenverteilung von Bund und Ländern zusammen und wirkt sich auf die Beschäftigtenstruktur aus. 34\% der österreichischen Bundesbediensteten sind im Verwaltungsdienst 
tätig. Die zweitgrößte Gruppe stellen mit 30\% BundeslehrerInnen dar - dies sind LehrerInnen, die in Bundesschulen (d.h. in Allgemeinbildenden höheren Schulen sowie jenen der Sekundarstufe II) unterrichten und die rund ein Drittel aller LehrerInnen umfassen. Im Exekutiv- und Militärdienst ist ein weiteres Drittel aller Bundesbediensteten tätig.

Betrachtet man den Frauenanteil der Beschäftigten differenziert nach Berufsgruppen, so übersteigt der Frauenanteil in allen Gruppen außer dem Exekutivdienst und dem Militärischen Dienst die 50\%-Marke. Besonders groß ist der Anteil der Frauen bei den LehrerInnen mit 59\%. Der vergleichsweise hohe Frauenanteil der Bundesbediensteten in Österreich ist also auch auf die Struktur des Bundesdienstes zurückzuführen (vgl. Abb. 6.3).

Abb. 6.3: Bundesbedienstete und Frauenanteil nach Berufsgruppen (2012)
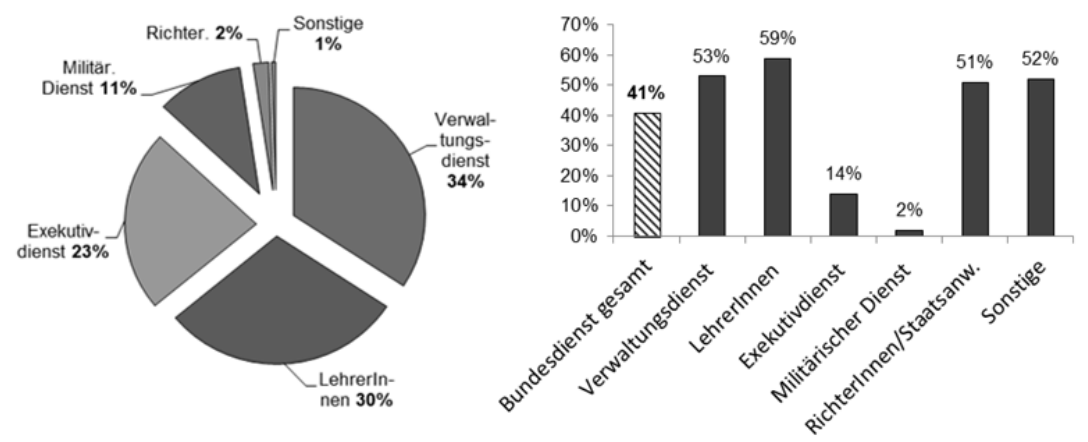

Quelle: eigene Darstellung auf Grundlage der Daten aus BKA (2013a: 25ff.)

Der Vergleich des Frauenanteils zwischen 2012 und 1995 nach Verwendungsgruppen zeigt deutliche Unterschiede bei der Feminisierung (Abb. 6.4). Während bei RichterInnen, StaatsanwältInnen, LehrerInnen, im Verwaltungsdienst und auch im Exekutivdienst der Frauenanteil deutlich gestiegen ist, zeigt sich wenig Veränderung im Militärdienst, der als nahezu reine Männerbastion mit nur 2\% Frauen weiterbesteht. Der überproportional hohe Anstieg bei den LehrerInnen weist darauf hin, dass Frauen nun auch verstärkt in Lehrberufen mit Universitätsabschluss tätig sind. ${ }^{6}$ Ein besonders hoher Anstieg ist in der Berufsgruppe „RichterInnen/StaatsanwältInnen“ (+20 Prozentpunkte) zu verzeichnen. Der Frauenanteil ist lediglich im Krankenpflegedienst gesunken, der aber kaum ins Gewicht fällt $(0,2 \%$ aller Bundesbediensteten).

6 In den Landes- und Gemeindeschulen, die Ausbildungen auf Ebene der Pädagogischen Akademien bzw. der Fachhochschulen voraussetzen, war der Frauenanteil schon immer deutlich höher. 
Abb. 6.4: Frauenanteil nach Beschäftigungsverhältnissen und Berufsgruppen im Bundesdienst (1995 und 2013)

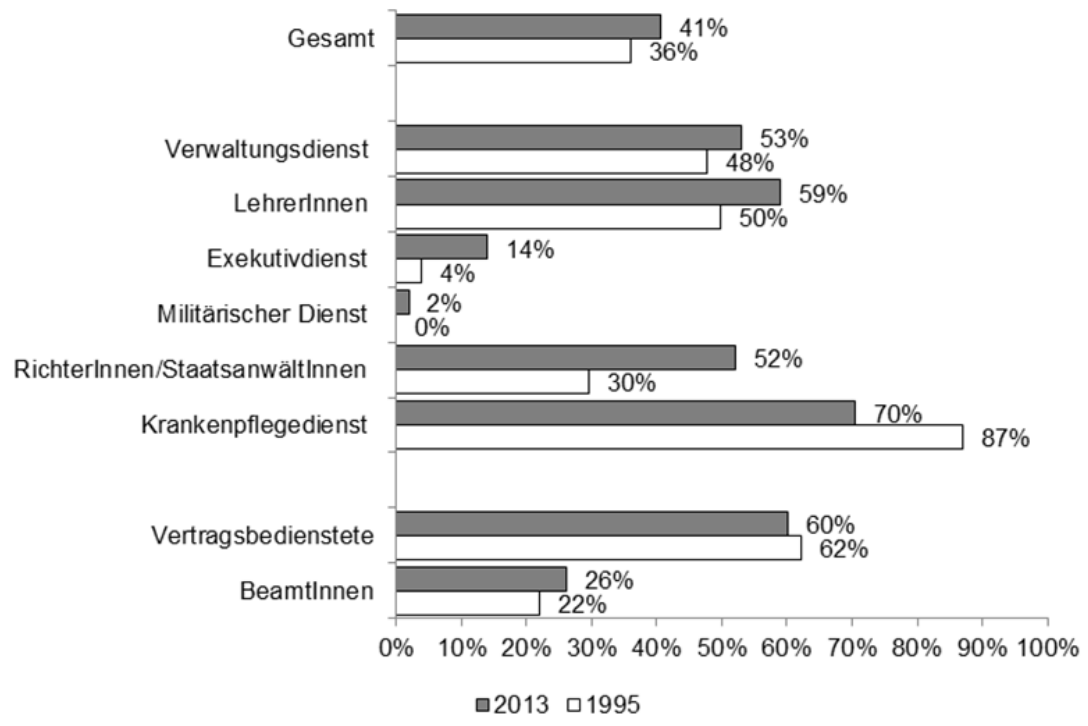

Quelle: eigene Darstellung auf Grundlage der Daten für 1995 aus BKA (2012: 50), für 2013 Verwendungsgruppen aus BKA (2014a: 18); Vertragsbedienstete und Beamte BKA (2013a: 57)

Frauen sind auch nach Beschäftigungsverhältnissen unterschiedlich verteilt: $60 \%$ der Vertragsbediensteten sind Frauen, aber nur 26\% der BeamtInnen. Auch dies hängt zumindest teilweise mit der unterschiedlichen Partizipation der Frauen nach Verwendungsgruppen zusammen. Vertragliche Dienstverhältnisse bestehen vor allem im Verwaltungsdienst und bei LehrerInnen, zwei Bereiche, in denen Frauen überproportional beschäftigt sind. Gegenüber 1995 ist der Frauenanteil bei BeamtInnen von $22 \%$ auf $26 \%$ gestiegen, während er bei den Vertragsbediensteten etwas gesunken ist.

\subsubsection{Auflösungs- und Beharrungstendenzen der vertikalen Segregation}

Der Bundesdienst stellt ein Beschäftigungsfeld mit einem großen Anteil von hoch qualifizierten Tätigkeitsbereichen dar (Abb. 6.5). Insgesamt ist das Qualifikationsniveau im Bundesdienst mit 33\% AkademikerInnenanteil und 18\% MaturantInnenanteil sehr hoch. Gerade in den höheren Qualifikationsklassen haben Frauen stark aufgeholt: Im Jahr 1995 waren sie mit 32\% Frauenanteil unter AkademikerInnen noch in der Minderheit, erreichten aber 2013 
einen Anteil von 55\%. Der geringe Frauenanteil im Bereich des Fachdienstes/Mittleren Dienstes (29\%) ist mit dem geringen Frauenanteil im Exekutivund Militärdienst verknüpft, die zwei Drittel dieser Qualifikationsgruppe umfassen. Im niedrigsten Qualifikationssegment ist der Frauenanteil mit 57\% nach wie vor überdurchschnittlich hoch. Dieser Gruppe kommt aber mengenmäßig - mit 4\% der Bundesbediensteten - eine geringe Bedeutung zu.

Abb. 6.5: Bundesbedienstete und Frauenanteil nach Qualifikationen (2013)
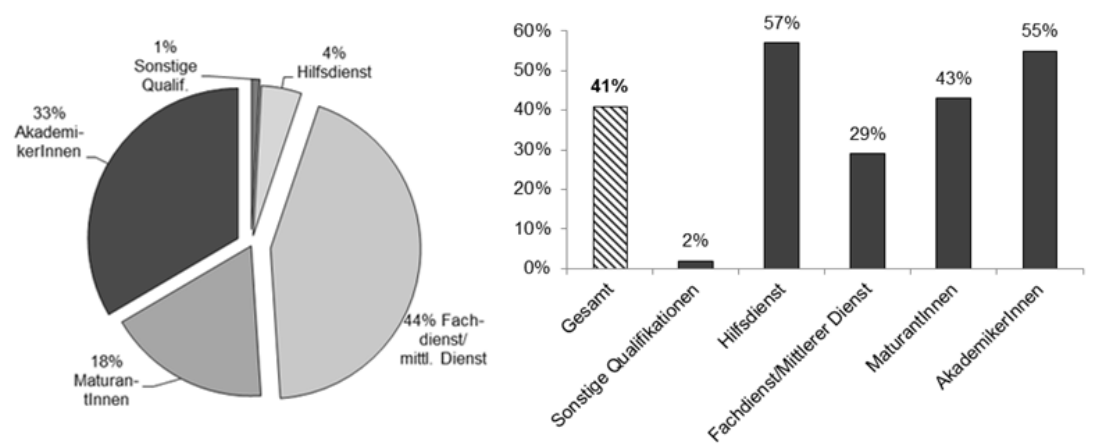

Quelle: eigene Darstellung auf Grundlage der Daten aus BKA (2014a: 41)

Die Gliederung der Tätigkeiten nach dem formalen Qualifikationsniveau der Bediensteten bildet entsprechend der engen Koppelung von formalen Bildungsabschlüssen, Stellenbesetzungen und Entlohnungssystem im Bundesdienst einen Indikator für die unterschiedliche Positionierung von Frauen und Männern. Aussagen zur Partizipation von Frauen in Führungspositionen und deren längerfristige Entwicklung erfordern einen genaueren Blick auf die Organisationsstruktur des Bundesdienstes. Prinzipiell gliedern sich die österreichischen Bundesministerien in Sektionen und diese in Abteilungen. Abteilungen können zu Gruppen zusammengefasst und in Referate untergliedert werden. Seit dem Jahr 2000 können zusätzlich GeneralsekretärInnen eingesetzt werden, die den Sektionen vorstehen und eine Zwischenebene nach der Ressortleitung (MinisterIn) bilden.

Nach dem Gleichbehandlungsbericht 2014 (BKA 2014a) ist keine der fünf Generalsekretariate mit einer Frau besetzt. Bei den SektionsleiterInnen (insgesamt 70 Stellen) liegt der Frauenanteil bei 23\%, er steigt bei den Gruppen- bzw. BereichsleiterInnen (insgesamt 111 Stellen) auf 28\% und bei den AbteilungsleiterInnen (insgesamt 522 Stellen) auf 37\%. Entsprechend der Analyse von Hackl und Steger-Mauerhofer (2012) ist der Frauenanteil in Führungspositionen (Sektions-, Gruppen-/Bereichs- und Abteilungsleitungen) in den Zentralstellen der Bundesministerien zwischen 1990 und 2010 
von $9 \%$ auf $28 \%$ gestiegen. ${ }^{7}$ Bei den Abteilungsleitungen betrug der Anstieg knapp 20 Prozentpunkte (von 10\% auf 30\%), bei den Sektionen und Gruppen rund 15 Prozentpunkte (von 3\% auf 18\%). Damit ist es innerhalb von 20 Jahren gelungen, den Frauenanteil in den Organisationsleitungen der Zentralstellen deutlich zu erhöhen.

Mit den genannten Positionen sind aber lediglich die Zentraleinheiten erfasst, während die nachgeordneten Dienststellen aufgrund ihrer organisatorischen Flexibilität schwerlich vergleichbar sind. Dementsprechend wird der Frauenanteil in Führungspositionen des Bundesdienstes gewöhnlich nach besoldungsrechtlichen Verwendungsgruppen ausgewiesen, die die unterschiedliche Größe von Sektionen, Gruppen, Bereichen und Abteilungen berücksichtigen und nachgeordnete Dienststellen beinhalten - dabei aber auch subjektive Bewertungsdimensionen enthalten.

Abb. 6.6: Frauenanteil in den höchsten besoldungsrechtlichen Einstufungen $(2006-2013$, in \%)

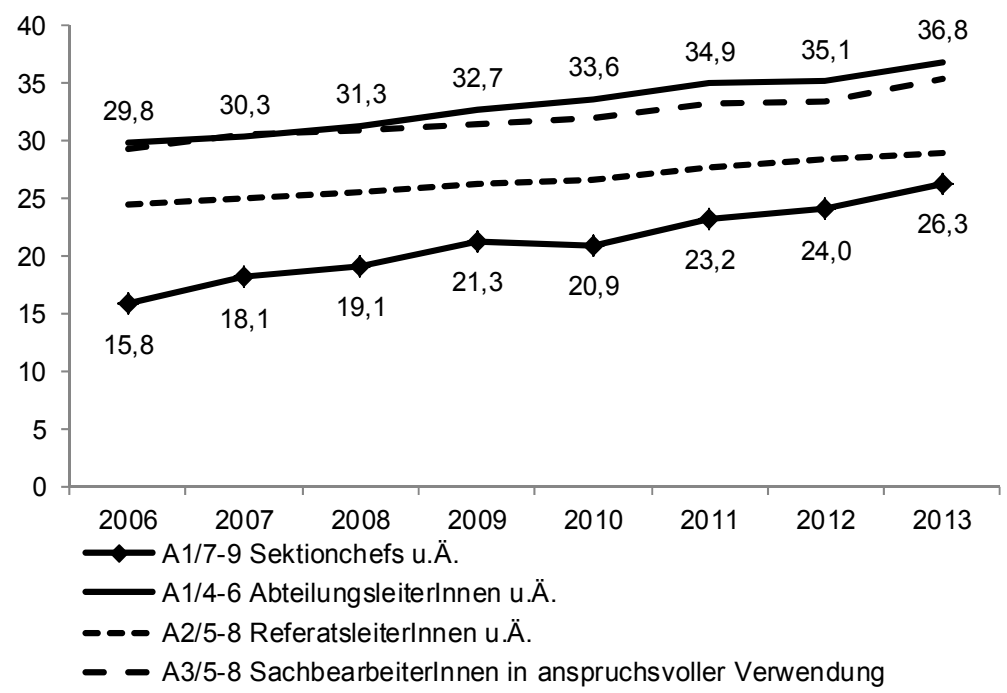

Quelle: BKA (2013a: 55)

Nach den besoldungsrechtlichen Einstufungen liegt der Frauenanteil in den höchsten Positionen, die 13\% der Bundesbediensteten erfassen, im Jahr 2013 bei 34\% (Abb. 6.6). Darin sind nicht nur Sektions-, Gruppen- und Abteilungsleitungen enthalten, sondern auch ReferatsleiterInnen, LeiterInnen größerer nachgeordneter Dienststellen sowie SachbearbeiterInnen ,in an-

7 Hackl und Steger-Mauerhofer verwendeten dafür die organisationsrechtlichen Positionen des österreichischen Amtskalenders. 
spruchsvoller Verwendung“". In den obersten Verwendungsgruppen A1/7-9 (Sektionsleitungen, GruppenleiterInnen und LeiterInnen großer nachgeordneter Dienststellen mit 0,3\% der Beschäftigten) liegt der Frauenanteil 2013 bei $26 \%$ und ist damit seit $2006 \mathrm{um}$ mehr als 10 Prozentpunkte gestiegen. Der Frauenanteil in den nachfolgenden Verwendungsgruppen A1/4-6 (AbteilungsleiterInnen, AbteilungsleiterInnen-StellvertreterInnen, ReferatsleiterInnen und LeiterInnen größerer nachgeordneter Dienststellen mit 3\% der Beschäftigten) ist mit $37 \%$ deutlich höher, auch höher als die nachfolgenden Verwendungsgruppen A2/5-8 (Referatsleitungen u.Ä. mit 4\% der Beschäftigten) mit $28 \%$ sowie A3/5-8 (SachbearbeiterInnen in anspruchsvoller Verwendung mit 6\% der Bediensteten) mit 33\% (BKA 2013a: 55).

Während die Karrieremöglichkeiten von Frauen in der Bundesverwaltung einen zentralen Stellenwert in der Gleichstellungsdiskussion einnehmen, waren Einkommensunterschiede zwischen Frauen und Männern bislang kaum ein Thema. Aufgrund der gesetzlichen Besoldungsschemata galt der öffentliche Dienst im Hinblick auf Einkommensunterschiede als geschlechtsneutral. Die Feststellung des Entgelts erfolgt sowohl bei BeamtInnen als auch bei Vertragsbediensteten auf Grundlage ihrer Verwendung, die die Aufgaben der Arbeitsplätze nach einem einheitlichen Schema bewertet. Leistungsorientierte Gehaltsbestandteile spielen mengenmäßig eine geringe Rolle und werden in den Interviews eher in ihrer symbolischen Bedeutung thematisiert. Insgesamt gilt sowohl für BeamtInnen als auch Vertragsbedienstete ein relativ starres Besoldungssystem, das jedoch durch die Bewertung des jeweiligen Arbeitsplatzes, durch Prämien und Zulagen und nicht zuletzt durch unterschiedliche Karrierewege zu unterschiedlichen Einkommensentwicklungen führen kann.

Mit Einführung der Einkommensberichtspflicht im Jahr 2011 ist die Diskussion über Einkommensunterschiede auch im öffentlichen Dienst gestiegen. ${ }^{8}$ Durch die Novellierung des Bundes-Gleichbehandlungsgesetzes im Jahr 2011 ist der Bundeskanzler bzw. die Bundeskanzlerin verpflichtet, jährlich einen Bericht zur Einkommensanalyse der DienstnehmerInnen des Bundes zu veröffentlichen. Er muss die Anzahl der Frauen und Männer in den jeweiligen Verwendungs-, Entlohnungs- und Gehaltsgruppen und das jeweilige Medianeinkommen enthalten. ${ }^{9}$

8 Parallel wurde auch eine zweijährige Einkommensberichtspflicht für privatwirtschaftliche Unternehmen ab einer gewissen Größe (seit 01.01.2014 Unternehmen mit mindestens 150 Beschäftigten) eingeführt. Dieser Bericht ist an den Betriebsrat zu übermitteln bzw., wenn kein Betriebsrat eingerichtet ist, den ArbeitnehmerInnen vorzulegen (vgl. BKA 2013a).

9 Der im Bericht zu verwendende Einkommensbegriff schließt Zulagen, Renumerationen, Sachbezüge und andere Entgeltbestandteile wie Bonuszahlungen ein. Das Arbeitsentgelt von Teilzeitbeschäftigten ist auf Vollzeitbeschäftigung und das von unterjährig Beschäftigten auf Jahresbeschäftigung hochzurechnen. Analog dazu werden Mehrstunden auf Vollzeit hochgerechnet. Der Einkommensbericht des Bundes enthält zusätzlich Informationen über 
Bislang liegen zwei Einkommensberichte für den Bundesdienst vor, die sich auf die Jahre 2013 und 2012 beziehen. Der um das Beschäftigungsausmaß bereinigte Gender Pay Gap betrug im Jahr 2013 13\% (Abb. 6.7). Er ist damit deutlich geringer als der Gender Pay Gap in der Privatwirtschaft (20\%). ${ }^{10}$ Gegenüber dem Jahr 2012 (13\%) ist der Gender Pay Gap um 0,5\%Punkte gesunken. ${ }^{11}$

Abb. 6.7: Gender Pay Gap im Bundesdienst nach Berufsgruppen (2013)

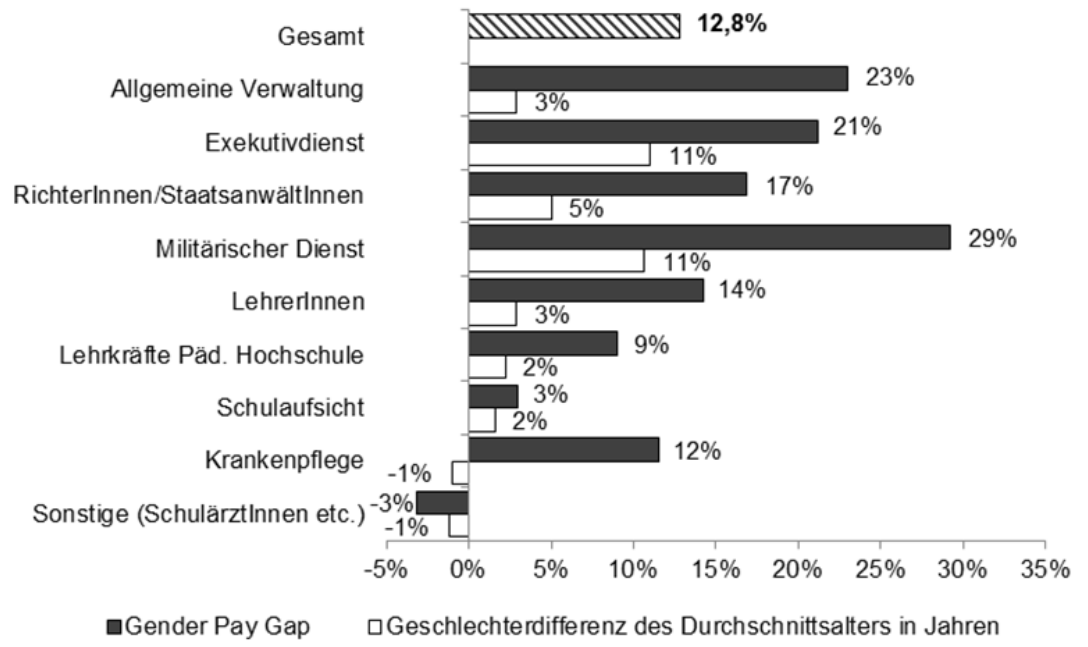

Quelle: BKA (2014b: 4ff.)

Vergleicht man den Gender Pay Gap nach Berufsgruppen zwischen Frauen und Männern, liegt der ausgewiesene Gender Pay Gap im Militärischen Dienst, in der Allgemeinen Verwaltung, dem Exekutivdienst, bei den RichterInnen und StaatsanwältInnen sowie den LehrerInnen über dem Durchschnittswert des Bundesdienstes. Die großen Einkommensunterschiede im Militär- und Exekutivdienst gehen mit einem relativ großen Unterschied des Durchschnittsalters von elf Jahren einher. Berufsgruppen, in denen der Gender Pay Gap unter 10\% liegt (Lehrkräfte der Pädagogischen Hochschulen, der

das biologische Durchschnittsalter von Frauen und Männern als Erklärung für Einkommensunterschiede.

10 Gender Pay Gap nach Stundenlöhnen der Voll- und Teilzeitbeschäftigten in Österreich auf Basis der Mikrozensus-Arbeitskräfteerhebung 2014 (Einkommensbericht des Rechnungshofes 2014).

11 Der im Gleichbehandlungsbericht 2012 ausgewiesene Einkommensunterschied für 2011 von $15 \%$ enthält lediglich vollzeitbeschäftigte Bedienstete und ist damit nicht unmittelbar vergleichbar. 
Schulaufsicht, und Sonstige wie z.B. ÄrztInnen), betreffen weniger als $2 \%$ der Bundesbediensteten.

Ähnlich wie der Frauenanteil des Bundespersonals wird auch der relativ geringe Gender Pay Gap wesentlich durch den hohen Anteil von LehrerInnen beeinflusst. Denn im frauendominierten Lehrdienst ist der Gender Pay Gap relativ niedrig, und die Einkommen sind überdurchschnittlich hoch. Deutlich höher, nämlich auch höher als in der Gesamtwirtschaft, ist der Gender Pay Gap in der Allgemeinen Verwaltung; er hat sich gegenüber 2012 auch nicht verringert. Die Erklärung dafür ist nicht in der Altersdifferenz zwischen Frauen und Männern zu finden (die weniger als drei Jahre beträgt), sondern ist auf unterschiedliche Qualifikationen und Einstufungen von Frauen und Männern in dem an sich fixen, auf Seniorität ausgerichteten Besoldungssystem und die weiterhin niedrige Präsenz von Frauen in Führungspositionen zurückzuführen.

\subsubsection{Unterschiede zwischen Ressorts}

Die Zusammensetzung der Bediensteten der einzelnen Ressorts ist so unterschiedlich wie die Aufgabenstellungen, die die verschiedenen Teile der Verwaltung haben. Deutlich wird dies schon an der unterschiedlichen Größe der 13 Bundesministerien (Tab. 6.1). ${ }^{12}$ Traditionell große Ressorts sind jene, die sich mit Bildungs- und Sicherheitsfragen befassen und dabei einen großen nachgeordneten Bereich aufweisen: Das Unterrichtsministerium beschäftigt mit rund 43.000 LehrerInnen 36\% aller Bundesbediensteten, das Innenministerium mit rund 27.000 Exekutivbediensteten $23 \%$, das Verteidigungsministerium mit knapp 14.000 Militärkräften 16\%. Justizministerium und Finanzministerium umfassen jeweils $8 \%$ der Bediensteten. Die weiteren acht Ressorts betreffen zwischen $0,3 \%$ (Gesundheitsministerium) und 2\% (Lebensministerium) der Bediensteten.

Entsprechend den unterschiedlichen Aufgabenfeldern variiert die Geschlechterstruktur zwischen den Ressorts erheblich. Während in BKA, BMASK, BMG, BMUKK und BMWF Frauen mit rund $60 \%$ eine deutliche Mehrheit der Bediensteten stellen, liegt ihr Anteil im BMLVS lediglich bei

12 Diese Ressortzusammensetzung ist eine Bestandsaufnahme von Mitte 2013. Sie hat sich mit den Regierungswechseln immer wieder verändert. Beispielsweise war das Bundesministerium für Wissenschaft und Forschung zwischen 2000 und 2007 dem Bundesministerium für Unterricht und Kunst zugeordnet. Frauenangelegenheiten waren über die Zweitzuständigkeit der MinisterInnen in unterschiedliche Ressorts eingebunden. Im Erhebungszeitraum waren Frauenangelegenheiten mit dem öffentlichen Dienst gekoppelt und im Bundeskanzleramt beheimatet. Davor waren sie Medien und Regionalpolitik, Gesundheit und vor der Auflösung des Frauenministeriums (2000 bis 2003) dem Konsumentenschutz beigeordnet. Ende 2013 sind die Frauenangelegenheiten dem Unterrichtsministerium zugeordnet worden. 
Tab. 6.1: Bedienstete und Frauenanteil nach Ressorts (2013)

\begin{tabular}{|c|c|c|c|c|c|c|c|}
\hline & 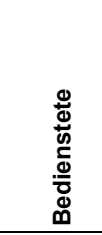 & 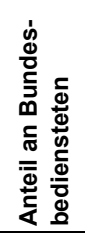 & 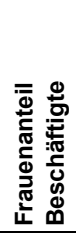 & 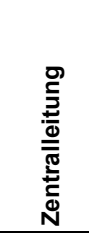 & 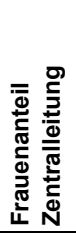 & 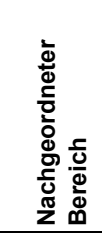 & 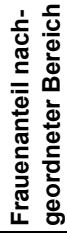 \\
\hline $\begin{array}{l}\text { Bundeskanzleramt } \\
\text { (BKA) }\end{array}$ & 1.001 & $1 \%$ & $61 \%$ & 681 & $64 \%$ & 320 & $56 \%$ \\
\hline $\begin{array}{l}\text { BM für europäische und } \\
\text { internationale Angele- } \\
\text { genheiten (BMeiA) }\end{array}$ & 1.171 & $1 \%$ & $48 \%$ & 539 & $47 \%$ & 632 & $50 \%$ \\
\hline $\begin{array}{l}\text { BM für Arbeit, Soziales } \\
\text { und Konsumenten- } \\
\text { schutz (BMASK) }\end{array}$ & 1.625 & $1 \%$ & $59 \%$ & 576 & $56 \%$ & 1.049 & $60 \%$ \\
\hline BM für Finanzen (BMF) & 11.065 & $8 \%$ & $48 \%$ & 729 & $47 \%$ & 10.336 & $48 \%$ \\
\hline $\begin{array}{l}\text { BM für Gesundheit } \\
\text { (BMG) }\end{array}$ & 367 & $0,3 \%$ & $62 \%$ & 367 & $62 \%$ & - & - \\
\hline BM für Inneres (BMI) & 32.085 & $23 \%$ & $20 \%$ & 1.763 & $37 \%$ & 30.322 & $20 \%$ \\
\hline BM für Justiz (BMJ) & 11.586 & $8 \%$ & $52 \%$ & 221 & $49 \%$ & 11.365 & $52 \%$ \\
\hline $\begin{array}{l}\text { BM für Landesverteidi- } \\
\text { gung und Sport } \\
\text { (BMLVS) }\end{array}$ & 21.535 & $15 \%$ & $11 \%$ & 924 & $32 \%$ & 20.611 & $10 \%$ \\
\hline $\begin{array}{l}\text { BM für Land- und } \\
\text { Forstwirtschaft, Umwelt } \\
\text { und Wasserwirtschaft } \\
\text { (BMLFUW) }\end{array}$ & 2.772 & $2 \%$ & $49 \%$ & 908 & $58 \%$ & 1.864 & $44 \%$ \\
\hline $\begin{array}{l}\text { BM für Unterricht, Kunst } \\
\text { und Kultur (BMUKK) }\end{array}$ & 50.667 & $36 \%$ & $61 \%$ & 664 & $65 \%$ & 50.003 & $61 \%$ \\
\hline $\begin{array}{l}\text { BM für Verkehr, Innova- } \\
\text { tion und Technologie } \\
\text { (BMVIT) }\end{array}$ & 828 & $1 \%$ & $43 \%$ & 582" & $47 \%$ & 246 & $36 \%$ \\
\hline $\begin{array}{l}\text { BM für Wirtschaft, } \\
\text { Familie und Jugend } \\
\text { (BMWFJ) }\end{array}$ & 2.299 & $2 \%$ & $39 \%$ & 933 & $59 \%$ & 1.366 & $26 \%$ \\
\hline $\begin{array}{l}\text { BM für Wissenschaft } \\
\text { und Forschung (BMWF) }\end{array}$ & 797 & $1 \%$ & $57 \%$ & 334 & $64 \%$ & 463 & $52 \%$ \\
\hline Bundesdienst Gesamt & 138.980 & $100 \%$ & $41 \%$ & 9.221 & $50 \%$ & 128.577 & $40 \%$ \\
\hline
\end{tabular}

Bedienstete auf Basis von Vollbeschäftigungsäquivalenten. Bundesdienst inkl. Oberste Bundesbehörden

Quelle: BKA (2014a: 28ff.) 


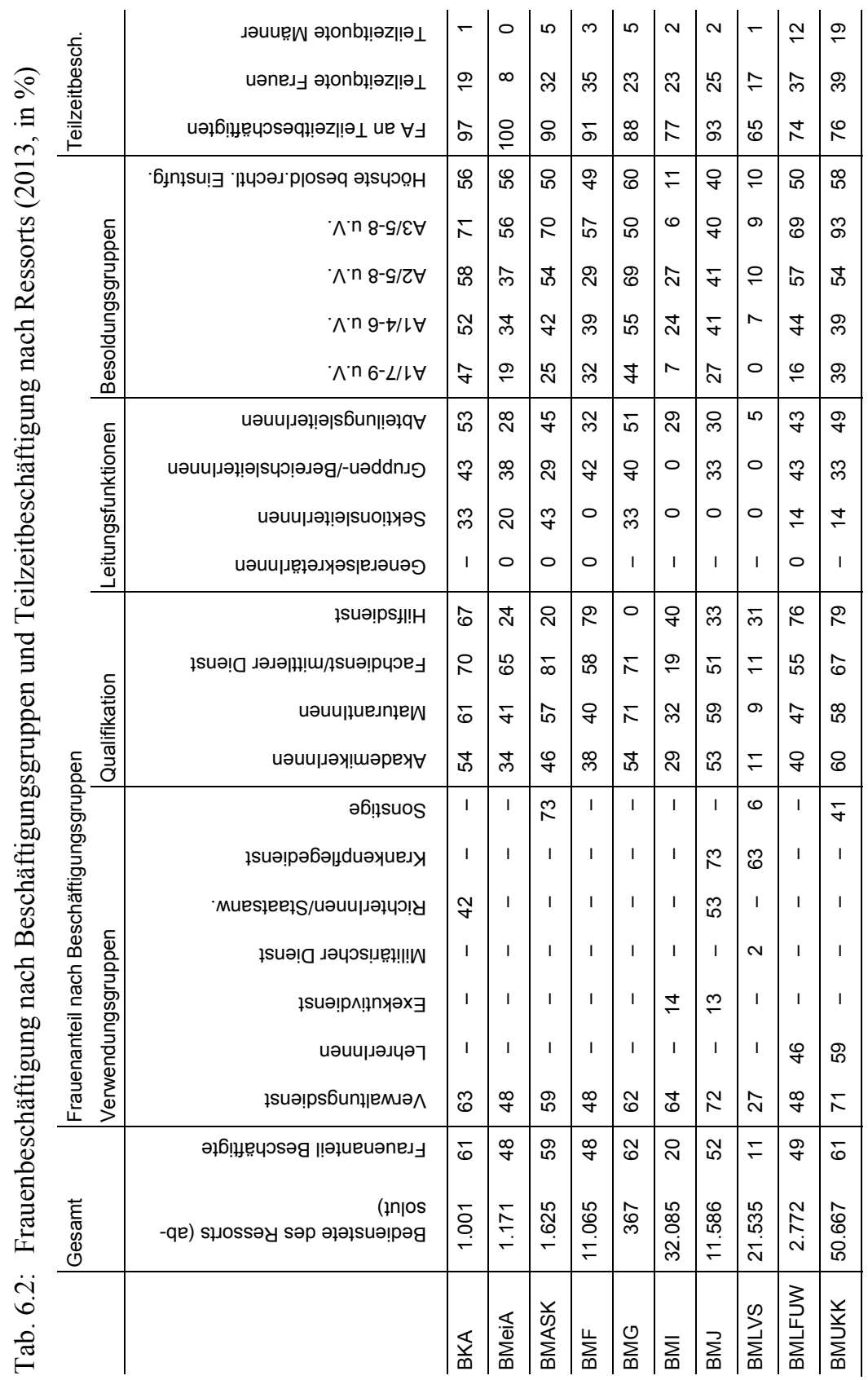




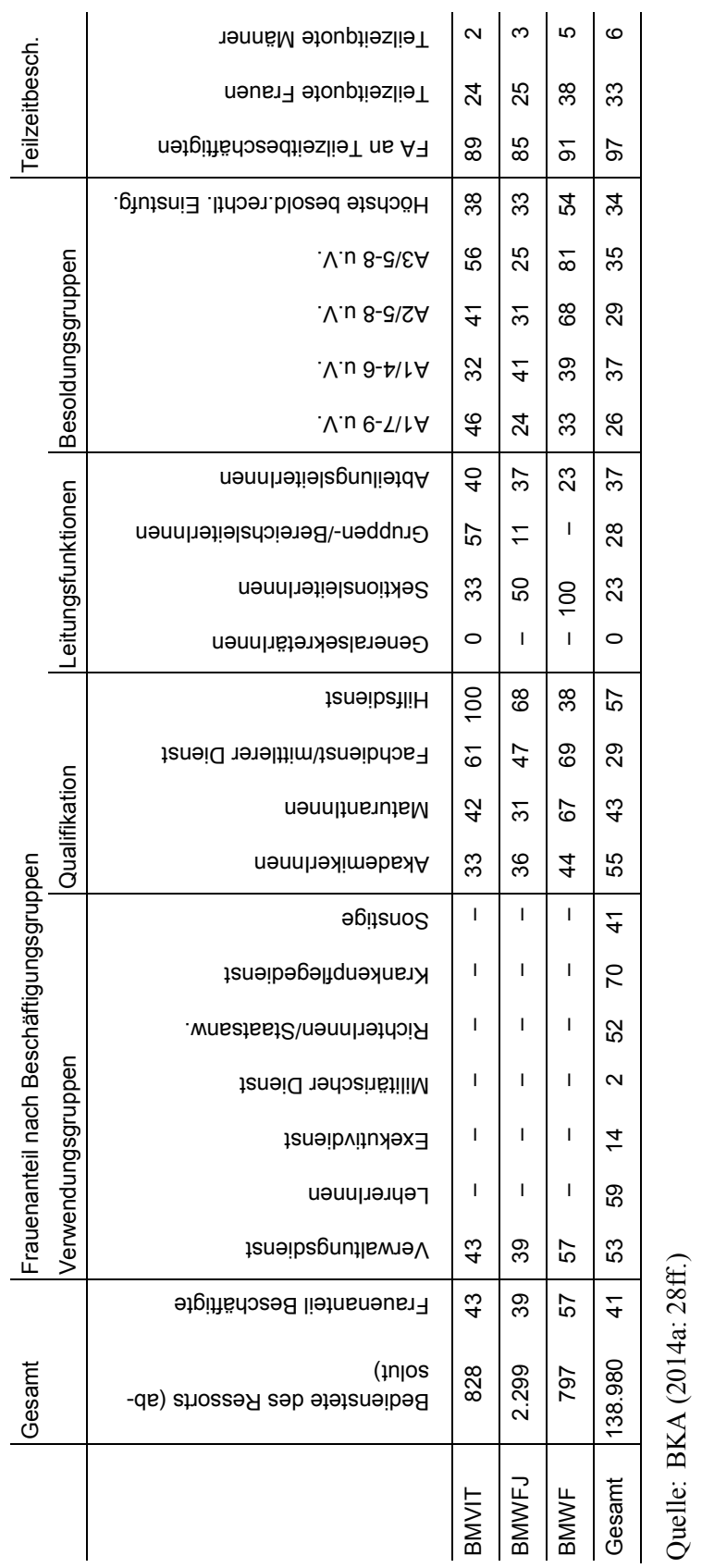


$11 \%$ und im BMI bei $20 \%$. In fast allen Ressorts liegt der Frauenanteil über jenem der Gesamtbeschäftigten Österreichs (46\% Frauenanteil), lediglich im Verteidigungsministerium wie auch im Innenministerium sind Frauen unterrepräsentiert. Dies betrifft nicht nur die nachgeordneten Stellen, sondern auch die Zentralleitung (BMI 37\%, BMLVS 32\% Frauenanteil in Zentralleitung).

Insgesamt unterscheiden sich die Geschlechteranteile in den nachgeordneten Bereichen deutlich stärker als in den Zentralleitungen. Das Wirtschaftsministerium weist neben dem Verteidigungs- und Innenministerium einen sehr geringen Frauenanteil in den nachgeordneten Dienststellen auf: Mit nur 23\% Frauenanteil im Bundesamt für Eich- und Vermessungswesen, dem größten nachgeordneten Bereich mit mehr als der Hälfte der Beschäftigten des Ressorts, bleibt der Frauenanteil sowohl im nachgeordneten Bereich (26\%) wie auch insgesamt im Wissenschaftsministerium (39\%) niedrig.

Wie die Differenzierung nach Ressorts zeigt, ist auch der hohe Frauenanteil bei AkademikerInnen durch den großen Anteil von LehrerInnen geprägt (Tab. 6.2). Im BMUKK ist der Frauenanteil bei AkademikerInnen mit $60 \%$ am größten, während nur noch in vier weiteren Ressorts Frauen die Mehrheit unter AkademikerInnen stellen (BKA, BMG, BMJ). In sechs Ministerien liegt der Frauenanteil unter AkademikerInnen hingegen unter 40\%. Frauen dominieren indessen mit Ausnahme des Innen- und Verteidigungsministeriums in allen Ressorts den Fachdienst bzw. mittleren Dienst. Das heißt, die Ressorts unterscheiden sich auch im Hinblick auf das Qualifikationsniveau von Frauen sehr stark.

Ein hoher Frauenanteil bei AkademikerInnen geht aber nicht unbedingt mit einer hohen Frauenpartizipation in Leitungsfunktionen einher. Nur im BMVIT ist der Frauenanteil an den Leitungspositionen höher als der von AkademikerInnen. In allen anderen Ressorts liegt der Frauenanteil von AkademikerInnen über, meist deutlich über jenem in Leitungspositionen. Diese Differenz steigt über die Hierarchien, denn der Frauenanteil in den Sektionsleitungen und Bereichsleitungen ist fast durchwegs geringer als bei Abteilungsleitungen - eine Ausnahme stellt auch hier das BMVIT dar. In den obersten Leitungsfunktionen der Ministerien, den Sektionsleitungen, gibt es in vier Ministerien keine Frau, in drei Ministerien einen Frauenanteil von maximal 20\% und nur in zwei Ministerien ist das Geschlechterverhältnis ausgeglichen bzw. stellen Frauen die Mehrheit.

Der Vergleich der Ressorts zeigt aber auch, dass der Frauenanteil in den höchsten besoldungsrechtlichen Einstufungen auf Ressortebene deutlich vom Anteil an den Leitungsfunktionen abweichen kann. In drei Ministerien beträgt der Frauenanteil in der obersten Besoldungsgruppe A1/7-9 über 40\%. Die betreffenden Ministerien (BKA, BMG und BMVIT) weisen zugleich hohe Frauenanteile in Leitungspositionen auf. Aber ein hoher Frauenanteil in den Leitungspositionen führt nicht unbedingt zu einem hohen Frauenanteil bei den höchsten Besoldungsgruppen, wie dies das Sozialministerium und 
Wirtschaftsministerium zeigen. Hingegen ist der Frauenanteil in den höchsten Besoldungsgruppen trotz relativ geringer Frauenpartizipation in Leitungsfunktionen im Finanzministerium und im Unterrichtsministerium hoch. Dies verweist auf die unterschiedliche Bewertung von Ressorttätigkeiten und nochmals mehr auf die Schwierigkeiten, den Frauenanteil in Entscheidungspositionen innerhalb der unterschiedlichen Organisationsstrukturen der Ministerien einheitlich zu definieren.

Die Teilzeitquoten korrelieren mit dem unterschiedlichen Frauenanteil der Ressorts, denn auch im Bundesdienst ist, wie bereits gezeigt, Teilzeitbeschäftigung überwiegend weiblich. Die höchsten Teilzeitquoten gibt es im Unterrichtsministerium (29\%), Wissenschaftsministerium (24\%), Lebensministerium (24\%) und Sozialministerium (21\%). Obwohl das Lebensministerium keinen überdurchschnittlich hohen Frauenanteil aufweist, ist die Teilzeitquote höher als z.B. im Bundeskanzleramt. Im Lebensministerium wie auch im Unterrichtsministerium sind auch die Teilzeitquoten bei Männern hoch, während im Sozialministerium, Finanzministerium und Wissenschaftsministerium die Teilzeitquote zwischen Frauen und Männern sehr stark variiert. In den männlich dominierten Ressorts, dem Verteidigungs- und Innenministerium, sind nur $1 \%$ bzw. $3 \%$ teilzeitbeschäftigt.

Die Auswahl der für Arbeit und Finanzen zuständigen Ressorts als Schwerpunktbereiche der Analyse erfolgte auf Basis der Schweizer Vorarbeiten mit dem Ziel, möglichst unterschiedliche Ministerien zu erfassen. In Österreich sind damit das Bundesministerium für Arbeit, Soziales und Konsumentenschutz (BMASK) und das Bundesministerium für Finanzen (BMF) betraut. Die beiden Ministerien unterscheiden sich deutlich hinsichtlich der Gleichstellungsrelevanz der inhaltlichen Tätigkeiten wie auch der Repräsentanz von Frauen:

Das Bundesministerium für Arbeit, Soziales und Konsumentenschutz ist mit seinen Kernbereichen Arbeitsrecht, Arbeitsschutz Arbeitsmarktpolitik, Sozialpolitik, Sozialversicherung, Seniorenpolitik sowie Pflege und Initiativen für Menschen mit Behinderung für originäre Bereiche der Gleichstellungspolitik zuständig. Rund ein Drittel der Beschäftigten sind der Zentralleitung zugeordnet. $\mathrm{Zu}$ den nachgeordnete Bereichen zählen das Arbeitsinspektorat (rund 400 Beschäftigte) und die Bundessozialämter (rund 600 Beschäftigte). Zwischen 2000 und 2009 war der Bereich Arbeit dem Ressort für Wirtschaft zugeordnet, während das Sozialressort mit dem Bereich Generationen und später mit dem Konsumentenschutz zusammengelegt wurde. Die Repräsentanz von Frauen ist in diesem Ressort sowohl auf Ebene der PolitikerInnen als auch der Verwaltung hoch. 1997 wurde nach mehreren Staatssekretärinnen für Soziales die erste Sozialministerin berufen und das Ressort seither für ein Drittel der Zeit von Ministerinnen geleitet. Mit 59\% bilden Frauen eine deutliche Mehrheit der Ressortbeschäftigten. Insbesondere im Fachdienst bzw. mittleren Dienst sind Frauen stark vertreten (81\%), während 
der Frauenanteil unter AkademikerInnen relativ gering ist (46\%). Das Sozialministerium weist einen der höchsten Frauenanteile bei Sektionsleitungen auf und einen überdurchschnittlich hohen Anteil bei Abteilungsleitungen. Der Frauenanteil in den höchsten Besoldungsgruppen liegt hingegen lediglich im Durchschnitt des Bundesdienstes und ist zwischen 2007 und 2013 weniger stark gestiegen als der Frauenanteil insgesamt (Abb. 6.8).

Das Bundesministerium für Finanzen übt mit Aufgaben der Budget- und Steuerpolitik zentrale Funktionen der öffentlichen Verwaltung aus. Gleichstellung ist in den Aufgaben des Ressorts eher ein Randthema, auch wenn das Thema durch Gender Budgeting und verpflichtende Gleichstellungsziele in der wirkungsorientierten Haushaltsführung an Bedeutung gewonnen hat. Mit den Zoll- und Finanzämtern weist das BMF einen großen nachgeordneten Bereich mit über 10.000 Beschäftigten auf, der sich hinsichtlich der Geschlechterstruktur aber kaum von der Zentralleitung unterscheidet. Zwischen 2011 und 2013 wurde das Finanzministerium erstmals von einer Frau geleitet, es gab aber bislang keine Sektionsleiterin im Finanzressort. Der Frauenanteil des BMF ist mit Ausnahme der Gruppen- bzw. BereichsleiterInnen auf allen Ebenen geringer als im BMASK. Der Anteil der Frauen in den höchsten Besoldungsgruppen ist in allen Kategorien höher als im Bundesdienst insgesamt und liegt in den obersten Verwendungsgruppen A1/7-9 aufgrund eines starken Anstiegs zwischen 2007 und 2009 nunmehr auch deutlich über dem Frauenanteil im BMASK (Abb. 6.8).

Abb. 6.8: Veränderung des Frauenanteils in den höchsten Besoldungsgruppen im Bundesdienst gesamt, BMASK und BMF (2007-2013)

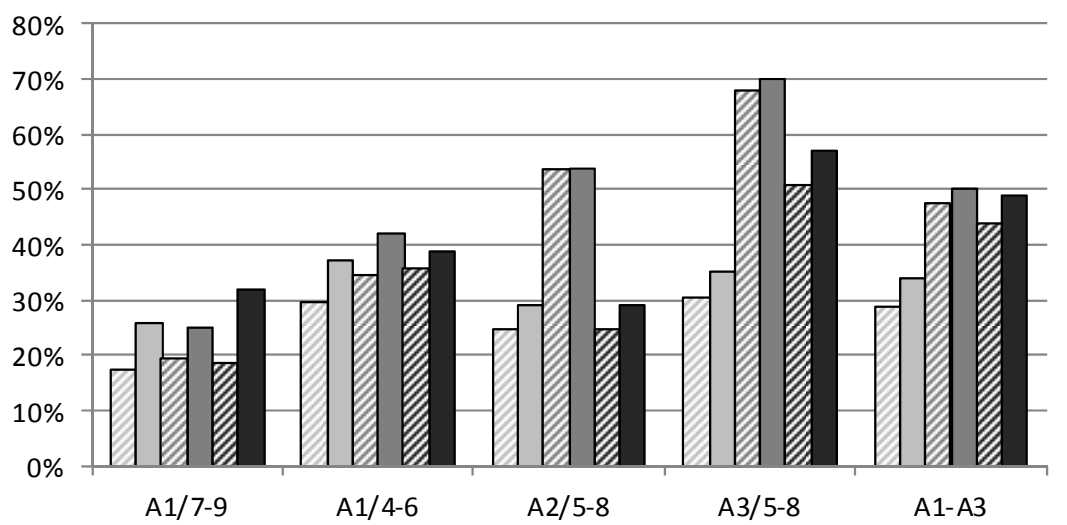

Gesamt $2007 \square$ Gesamt 2013 — BMASK 2007

BMASK 2013 BMF 2007 aMF 2013

Quelle: eigene Darstellung auf Grundlage der Daten aus BKA (2014a: 55, 2010: 20) 
Im Hinblick auf gleichstellungspolitische Maßnahmen und Aktivitäten zeigen sich aber beide Ministerien sehr engagiert, wie in den folgenden Abschnitten noch näher beschrieben.

\subsubsection{Zwischenfazit: Segregierte Feminisierung}

Der Frauenanteil der Beschäftigten im Bundesdienst ist in Österreich in den letzten Jahrzehnten kontinuierlich gestiegen. Doch diese Feminisierung erfolgte nicht in allen Bereichen des Bundesdienstes gleichermaßen. Zwischen Ressorts und Verwendungsgruppen, vor allem zwischen den Hierarchieebenen, bestehen erhebliche Unterschiede der Frauenpartizipation, es kam also zu einer segregierten Feminisierung.

Die Bandbreite des Frauenanteils der Bundesbediensteten liegt zwischen $11 \%$ und $61 \%$ in den einzelnen Ressorts. Diese Spaltung hängt mit den unterschiedlichen Aufgabenbereichen der Ressorts und der Segregation nach Berufsgruppen zusammen. Der im Vergleich zu Deutschland und der Schweiz deutlich höhere Frauenanteil in Österreich von $41 \%$ sinkt auf $33 \%$, wenn LehrerInnen, die in keinem der anderen Länder dem Bundesdienst angehören, herausgerechnet werden. Hingegen ist der Frauenanteil mit 14\% im Exekutivdienst und nur 2\% im Militärdienst deutlich geringer als in den beiden anderen Ländern (vgl. Kapitel 3 in diesem Buch).

Beharrlich bleibt die Spaltung vor allem in den obersten Hierarchiestufen. Frauen sind in den Leitungsfunktionen der Ministerien mit 34\% deutlich weniger vertreten als in den anderen Positionen der Zentraleinheiten (51\%). Generalsekretariate, die in fünf Ressorts als oberste Ebene bestehen, sind durchgehend männlich besetzt, und in einigen Ressorts, im Finanz-, Innenund Verteidigungsministerium gibt es nach wie vor keine weiblichen Sektionsleitungen. In den darunter liegenden Leitungsebenen, den Gruppen- und Bereichsleitungen, sind im Innenministerium, Verteidigungsministerium, Wirtschafts- und Familienministerium keine Frauen vertreten. Höher ist die Repräsentanz von Frauen auf Ebene der Leitungsfunktionen hingegen im Bundeskanzleramt, im Gesundheitsministerium sowie im Verkehrsministerium.

Im Sozialministerium hat sich die Frauenpartizipation gegenüber den 1980er Jahren erheblich verändert, die Rowhani (1991: 196) in den obersten Hierarchien noch als weitgehend „frauenfrei“" beschreibt und dies auf Tradition oder auch offen zur Schau getragene Frauenverachtung zurückführt. Frauen haben im Sozialressort auch in den Leitungsfunktionen mit $41 \% \mathrm{mehr}$ als eine kritische Masse erreicht. Im Finanzressort liegt der Frauenanteil bei $32 \%$, also etwas unter dem durchschnittlichen Frauenanteil aller Ministerien von 34\%. Aber die unterschiedliche Repräsentanz von Frauen lässt vermuten, dass Tradition, vielleicht in Einzelfällen auch „Frauenverachtung“ (ebd.) in 
Teilen weiterwirken, sodass Frauen trotz - des Bekenntnisses zu einer geschlechtergerechten Partizipation - auf allen Ebenen und Positionen gänzlich fehlen oder eine kleine Minderheit bleiben.

Offensichtlich wird die unterschiedliche Positionierung von Frauen und Männern im Gender Pay Gap. Im Kernbereich des Bundesdienstes, der allgemeinen Verwaltung, ist der Einkommensunterschied zwischen Frauen und Männern mit 23\% gleich hoch wie in der Gesamtwirtschaft. Der durchschnittliche Gender Pay Gap im gesamten Bundesdienst ist mit 13\% auch deshalb niedriger, als die großen Männerbastionen des Exekutiv- und Militärdienstes niedrigere Qualifikationen und Einkommen aufweisen als der frauendominierte Bereich der Lehrkräfte. Interessant ist aber auch der $\mathrm{Zu}$ sammenhang zwischen der horizontalen Segregation und der Bewertung der Tätigkeiten. Denn der Vergleich des Frauenanteils in Leitungspositionen und in den höchsten besoldungsrechtlichen Verwendungsgruppen zeugt davon, dass innerhalb des Bundesdienstes eine differenzierte Bewertung von Fachgebieten stattfindet, indem die wirtschaftlichen Bereiche, zu denen auch der Finanzbereich zählt, einen größeren Anteil in den höchsten Besoldungsgruppen aufweisen. Damit ist im BMF trotz der geringeren Frauenpräsenz in Leitungspositionen der Frauenanteil in den obersten Besoldungsgruppen mit $32 \%$ größer als im BMASK (25\%).

Die 1991 von Rowhani beschriebenen offenen Bekenntnisse von Vorgesetzten, „dass sie keine Frauen aufnehmen oder höchstens solche, die keine Kinder mehr bekommen", gibt es heute kaum mehr. Frauendiskriminierung ist subtiler geworden, aber es werden bei der Gleichbehandlungskommission jedes Jahr eine ganze Reihe von Anträgen wegen Ungleichbehandlung von Frauen und Männern eingebracht (zwischen März 2012 und Februar 201450 Anträge von Frauen).

\subsection{Institutionelle Politik im österreichischen Bundesdienst}

Maßnahmen und Institutionen für die Gleichstellung von Frauen und Männern sind im Bundesdienst in Österreich stark formalisiert. Die Institutionalisierung der Gleichstellungspolitik des Bundes erfolgte in mehreren Schritten, die durch internationale Organisationen, allen voran die EU, mitbestimmt und/oder von nationalen AkteurInnen getragen wurde. Mit der Ratifizierung internationaler Abkommen zur Gleichstellung, wie der Konvention zur Beseitigung jeder Form von Diskriminierung der Frau (CEDAW), der Europäischen Sozialcharta oder den ILO-Übereinkommen zur Verwirklichung von Gleichbehandlung No. 100, wurden Rahmenbedingungen festgelegt. Der Europäisierungsprozess, d.h. die Implementierung von gemeinschaftlichen Verträgen und Gesetzen, hat in Österreich zur Institutionalisierung der 
Gleichstellungspolitik beigetragen (Rosenberger 2009). Dies betrifft auch Regelungen im Vorfeld des EU-Beitritts im Jahr 1995, in dessen Phase auch die Installierung des Gleichbehandlungsgesetzes des Bundes fällt. Vor allem nach 2000 gehen fast alle institutionellen Einrichtungen auf Bundesebene auf Initiativen der EU zurück. Beispielsweise wurden die Ministerratsbeschlüsse zu einem Gender-Mainstreaming-Arbeitsprogramm für alle Ressorts (2002), zur Umsetzung von Gender Budgeting (2004) oder das Gleichbehandlungsgesetz (2004) für Diskriminierungsschutz bei den erweiterten Diskriminierungsgründen in Reaktion auf EU-Bestimmungen umgesetzt. Von Rosenberger (2009) wird die Gleichstellungspolitik zwischen 2000 und 2005 als „Umsetzungs- oder Befolgungspolitik“ beschrieben, indem europäische Initiativen die Institutionalisierung auf administrativer Ebene bestimmten. Erst ab dem Jahr 2009 werden mit den Diskussionen zur Einkommenstransparenz und dem Frauenanteil in Führungspositionen neue Initiativen zur nationalen Gleichstellungspolitik implementiert, die auch für den Bundesdienst relevant sind.

Zentrale Weichenstellungen für die Gleichstellungspolitik im Bundesdienst wurden in Österreich bereits sehr früh veranlasst und maßgeblich von der Staatssekretärin und späteren Frauenministerin Johanna Dohnal vorangetrieben. Dem Bundes-Gleichbehandlungsgesetz ist ein Frauenförderprogramm für den Bundesdienst (1981) vorausgegangen, das eine Grundlage für ressortspezifische Aktionspläne darstellte und erste strukturelle Maßnahmen wie die Installierung von „Kontaktfrauen“ als Vorläuferinnen der Gleichbehandlungsbeauftragten implementierte. In der Evaluierung dieses Programms wird die ,innovative Kraft“ des Frauenförderprogramms als zentrale Erklärung für den rasch zunehmenden Frauenanteil vor allem in den Jahren 1983 bis 1985 gesehen und eine zunehmende Akzeptanz von Frauenförderpolitik attestiert (Kreisky/Walther 1990). Es werden aber auch große Unterschiede zwischen den Ministerien thematisiert, die sich sowohl in der Formulierung der Aktionspläne als auch in den damit erreichten Ergebnissen niederschlagen. Als Handlungsbedarf für eine Verbesserung der Frauenförderung im Bundesdienst wird u.a. die gesetzliche Institutionalisierung der Frauenförderung aufgezeigt, um damit mehr Realisierungskraft im Durchsetzungsprozess zu erreichen. Des Weiteren wird eine grundsätzliche Reformbedürftigkeit der Rolle der Kontaktfrauen konstatiert. Ihre Verrechtlichung und Institutionalisierung wird grundsätzlich in zwei Richtungen gedacht, entweder in Richtung einer Verwaltungsaufgabe, d.h. in Richtung eines rechtlichen oder organisatorischen Status von Personalabteilungen, oder in Richtung einer weiblichen Interessensvertretung, also dem Modell der Personalvertretung folgend. 


\subsubsection{Bundes-Gleichbehandlungsgesetz als Rahmen der Gleichstellungspolitik}

Aufbauend auf den Zielsetzungen und der Bewertung des Förderprogramms für Frauen wurde im Jahr 1993 das Bundes-Gleichbehandlungsgesetz (B-GlBG) implementiert, das wegweisend für die Gleichstellungspolitik im Bundesdienst ist (BGBl. Nr. 100/1993 idgF). Das Bundes-Gleichbehandlungsgesetz wurde durch die Erfordernisse des EU-Rechts bzw. nationale Schwerpunktsetzungen mehrfach novelliert, indem beispielsweise 2004 eine Ausweitung der Gleichbehandlung neben dem Geschlecht auch in Bezug auf ethnische Zugehörigkeit, Religion und Weltanschauung, Alter oder sexuelle Orientierung erfolgte und 2011 der Bund verpflichtet wurde, jährlich einen Einkommensbericht zu erstellen, um die Einkommenstransparenz zu erhöhen.

Im Gleichbehandlungsgesetz sind vor allem Vorgaben zu institutionellen Maßnahmen der Gleichstellungspolitik enthalten. Durch die Einrichtung von Gleichbehandlungsbeauftragten, der Interministeriellen Arbeitsgruppe für Gleichbehandlungsfragen und der Bundes-Gleichbehandlungskommission sowie der Verpflichtung zur regelmäßigen Berichterstattung über den Stand der Verwirklichung der Gleichstellung soll eine Gleichbehandlung von Frauen und Männern sowie eine Frauenförderung bei bestehender Benachteiligung gesichert werden.

Inhaltliche Vorgaben zur Frauenförderung bestehen nur hinsichtlich der Beseitigung der Unterrepräsentanz von Frauen. Um ein ausgeglichenes Geschlechterverhältnis in allen Funktionen zu erreichen, ist ein Frauenfördergebot, d.h. eine Frauenquote im Sinne einer „Vorrangregel für Frauen“ definiert, die bis zum Erreichen der angestrebten Quote gilt. Konkret sind Frauen so lange bevorzugt aufzunehmen, wie sie in der jeweiligen Funktion unterrepräsentiert sind, d.h. der Frauenanteil unter 40\% bzw. 50\% liegt. ${ }^{13}$ Die Ausgestaltung substanzieller Maßnahmen erfolgt hingegen dezentral, d.h. obliegt den einzelnen Ressorts, die schwerpunktmäßig in deren Frauenförderplänen spezifiziert werden sollen.

\subsubsection{Frauenförderpläne als , soft laws“}

Mit den Frauenförderplänen der Ressorts sollen laut B-GlBG verbindliche Vorgaben für die Erhöhung des Frauenanteils in Verwendungs- und Besoldungsgruppen mit niedrigem Frauenanteil geschaffen werden. Die Beseitigung der Unterrepräsentanz der Frauen in spezifischen Funktionen (in höherwertigen Verwendungsgruppen wie auch in männerdominierten Berufsgruppen) ist damit als zentrale Zielsetzung definiert, die sich auf eine Verän-

13 Mit einer Novelle des Bundesgleichbehandlungsgesetzes im Jahr 2009 erfolgte zunächst eine Anhebung auf 45\% und 2011 auf 50\%. 
derung der Situation aller Frauen niederschlagen soll. Die Frauenförderpläne werden für jeweils sechs Jahre auf Vorschlag der Arbeitsgruppe für Gleichbehandlungsfragen von der Leitung der Zentralstelle erlassen und im Bundesgesetzblatt veröffentlicht. Mittels qualitativer Zielsetzungen und Vorgaben werden Schwerpunkte der Gleichstellungspolitik der Ressorts formuliert, um Benachteiligungen im Zusammenhang mit den Dienstverhältnissen entgegenzuwirken. Darüber hinaus werden für jeweils zwei Jahre quantitative Vorgaben für die Erhöhung des Frauenanteils nach Besoldungsgruppen, Funktionsgruppen oder Verwendungen formuliert. Wesentlich für seine Wirkungsmacht ist ein für alle Ressorts einheitliches Monitoring, das alle zwei Jahre in Form des Gleichbehandlungsberichts des Bundes veröffentlicht wird. Neben dem Monitoring von quantitativen Indikatoren zur Partizipation von Frauen sind darin auch Vorschläge zum Abbau der Benachteiligungen von Frauen sowie ein Bericht über die Tätigkeiten der Gleichbehandlungskommission des Bundes enthalten.

Die Ausgestaltung der Frauenförderpläne erfolgt in den einzelnen Ministerien sehr unterschiedlich. Dies betrifft sowohl den Umfang und damit die Ausdifferenziertheit der Regelungen als auch die inhaltlichen Schwerpunkte. Neben Themen zur Vereinbarkeit von Familie und Beruf, die beispielsweise Arbeitszeitregelungen, Kontakte und Rückkehr nach Berufsunterbrechungen betreffen, liegt ein weiterer Schwerpunkt der Maßnahmen bei der Karriereförderung von Frauen, d.h. auf Frauenquoten und Laufbahnplanung. Weiterhin werden Maßnahmen zur sprachlichen Gleichbehandlung und zur Vermeidung von sexueller Diskriminierung angeführt. In den Frauenförderplänen sind aber auch ressortspezifische Festlegungen zur Verankerung von Frauenförderung in der Personalplanung und Organisationsentwicklung und zur Unterstützung der Arbeit der Gleichbehandlungsbeauftragten sowie Informationsrechte enthalten.

Offensichtliche Unterschiede bestehen zwischen den männerdominierten Ressorts (BMI, BMVS) und den Ressorts mit stärkerem Gleichstellungsbezug wie BMASK oder BMUKK. Während Erstere durch Regelungen für die Vereinbarkeit von Familie und Beruf, insbesondere auch für die Beschäftigung in den nachgeordneten Dienststellen geprägt sind (spezifische Arbeitszeitregelungen durch Schicht- und Wechseldienst im Hinblick auf Familienverträglichkeit), werden bei den Letzteren stärker Vorschläge zur Karriereförderung und die Einbindung der Gleichbehandlung und Frauenförderung in der Organisationsentwicklung thematisiert. Die Unterschiede bestehen dabei sowohl im Hinblick auf die angesprochenen Themen als auch auf die Ausdifferenziertheit der Vorgaben in den Formulierungen. Trotz der Veröffentlichung der Frauenförderpläne erfolgte aber bislang keine vergleichende Analyse zwischen den Ressorts, und selbst für die Gleichbehandlungsbeauftragten sind die Unterschiede zwischen den Ressorts schwer fassbar, wie sich in den Interviews mit ExpertInnen gezeigt hat. 
Die für die Analyse ausgewählten Ressorts, das Finanzministerium und das Sozialministerium, zeichnen sich durch sehr ausdifferenzierte Frauenförderpläne aus. Im Frauenförderplan des Finanzministeriums werden Zielvorgaben zur Erhöhung des Frauenanteils entsprechend dem bestehenden Frauenanteil unabhängig von konkreten Beschäftigungspositionen definiert. ${ }^{14} \mathrm{Als}$ konkrete Vorschläge zum Abbau der Benachteiligung von Frauen werden Förderungen beim Wiedereinstieg (z.B. durch die Maßnahme „KarriereKompassKarenz") sowie Maßnahmen zur Qualifizierung (Erhöhung des Frauenanteils bei Qualifizierungen, genderkompetente Bildungseinrichtungen, Sensibilisierung von Führungskräften für Genderaspekte) angesprochen. Sowohl in den Zielsetzungen als auch bei den Maßnahmen werden Gleichbehandlung und Frauenförderung als Teil der Organisations- und Personalentwicklung postuliert.

Im Frauenförderplan des Sozialministeriums sind die Zielvorgaben zur Erhöhung des Frauenanteils für unterschiedliche Funktionsgruppen entsprechend der erwarteten Fluktuation definiert. ${ }^{15}$ Bei den substanziellen Maßnahmen werden Qualifizierungen von Teilzeitbeschäftigten und Wiedereinsteigerinnen sowie der (gleitende) Wiedereinstieg ebenfalls explizit als Förderziele hervorgehoben. Stärker als in anderen Ressorts wird bei der Vereinbarkeit von Familie und Beruf die Rolle von Männern thematisiert, um diese verstärkt einzubeziehen. Konkret geregelt ist im Frauenförderplan des BMASK aber auch das Informationsrecht der Arbeitsgruppe für Gleichbehandlungsfragen. Zudem sind Gleichbehandlung und Frauenförderung in der Organisations- und Personalentwicklung verankert, und es werden Prozessaspekte bei Begutachtungskommissionen, Mentoring als integrativer Teil der Personalplanung und -entwicklung, personelle und organisatorische Maßnahmen zur Unterstützung von Frauen und Männern mit Betreuungsverantwortung und die Vorbildfunktion der VertreterInnen der Personalabteilung hervorgehoben. Die Integration von Gleichbehandlung und Frauenförderung in der Organisations- und Personalentwicklung ist außer im Finanz- und Sozialministerium nur noch im Gesundheitsministerium verankert. In allen drei Ressorts sind Gleichbehandlungsbeauftragte zur Teilnahme an Bewerbungsverfahren einzuladen.

14 Beispielsweise wird für Positionen mit einem Frauenanteil bis 35\% im Dezember 2013 eine Erhöhung auf 50\% im Jahr 2017 angestrebt.

15 Zum Beispiel soll von den erwarteten Besetzungen eine Stelle in den obersten Verwendungsgruppen a1/7-a1/9 mit einer Frau besetzt werden, die zumindest gleich geeignet ist wie der bestgeeignete männliche Bewerber, in den Verwendungsgruppen A1/5, A1/6, v1/4 soll von zwei Fluktuationen eine Stelle mit einer Frau besetzt werden. 


\subsubsection{Gleichbehandlungsbeauftragte als individuelle AkteurInnen}

Pro Ressort gibt es mindestens drei und maximal sieben Gleichbehandlungsbeauftragte, die für fünf Jahre direkt von der Ressortleitung bestellt werden und gemeinsam die Arbeitsgruppe für Gleichbehandlungsfragen des Ressorts bilden. Die/der Vorsitzende der Arbeitsgruppe ist Mitglied der Interministeriellen Arbeitsgruppe für Gleichbehandlungsfragen, der neben den Vorsitzenden der Ressorts auch Mitglieder bundesnaher Organisationen wie Arbeitsmarktservice Österreich, Rechnungshof, Verfassungsgerichtshof, Verwaltungsgerichtshof, Volksanwaltschaft, Österreichische Post AG, Gewerkschaft öffentlicher Dienst, Statistik Austria, Bundesrechenzentrum, Bundestheater oder ARGE GLUNA angehören. Die Arbeitsgruppe untersteht der Frauenministerin. Darüber hinaus gibt es an allen Dienststellen des Bundes Kontaktfrauen bzw. Frauenbeauftragte. ${ }^{16}$

De jure sind Gleichbehandlungsbeauftragte für alle Fragen betreffend Gleichbehandlung von Frauen und Männern, Frauenförderung, Gleichbehandlung ohne Unterschied bezüglich der ethnischen Zugehörigkeit, der Religion oder der Weltanschauung, des Alters oder der sexuellen Orientierung im Ressort zuständig (B-GlBG § 29). Mit der Ausweitung der Gleichbehandlungsfragen auf weitere Diskriminierungsaspekte hat sich das Aufgabengebiet der Gleichbehandlungsbeauftragten stark vergrößert. Der Schwerpunkt ihrer Arbeit bleibt aber die Benachteiligung aufgrund des Geschlechts. ${ }^{17}$

Gleichbehandlungsbeauftragte erstellen im Auftrag der Ressortleitung den Frauenförderplan und stehen den Ressortleitungen beratend zur Seite. Die Schwerpunkte ihrer Tätigkeit sind in den Frauenförderplänen konkretisiert, wobei sich erhebliche Unterschiede zwischen den Ressorts zeigen. So ist in den beiden untersuchten Ressorts vorgesehen, dass Gleichbehandlungsbeauftragte als beratende Stimme (BMF) bzw. nicht stimmberechtige Sachverständige (BMASK) an Bewerbungsgesprächen und Auswahlhearings teilnehmen. Diese Ressorts nehmen damit - gemeinsam mit dem Gesundheitsministerium - eine Sonderstellung ein.

Eine Klärung des Verdachts auf Verletzung des Gleichbehandlungsgebots kann über die Gleichbehandlungskommission beantragt werden. Die Gleich-

16 An jeder Dienststelle des Bundes mit mindestens fünf Dienstnehmerinnen kann eine Kontaktfrau (Frauenbeauftragte) bestellt werden; und zwar von der bzw. dem Vorsitzenden der Arbeitsgruppe. Die Kontaktfrauen behandeln alle Anfragen, die Gleichbehandlung und Frauenförderung betreffen, sowie Wünsche oder Beschwerden der Dienstnehmerinnen. Auch sie sind in Ausübung ihrer Tätigkeit selbstständig und unabhängig. Die Zahl der Kontaktfrauen übersteigt jene der Gleichbehandlungsbeauftragten. So sind beispielsweise im BMASK neben der Vorsitzenden des Arbeitskreises für Gleichbehandlungsfragen sieben Gleichbehandlungsbeauftragte und 21 Kontaktfrauen tätig.

17 Beschwerden wegen Ungleichbehandlungen aufgrund des Alters werden nach Angaben der Interviewten zunehmend eingebracht. 
behandlungskommission stellt eine auch vom Arbeitskreis für Gleichbehandlungsbeauftragte unabhängige Beschwerdekommission dar, die aber deren Arbeit wirksam unterstützt. Der Großteil der Anträge (123 der 157 Gutachten) zwischen 2005 und 2012 bezieht sich auf die Gleichbehandlung von Frauen und Männern. Das heißt, auch nach Ausweitung des Gleichbehandlungsbereichs auf ethnische Zugehörigkeit, Religion und Weltanschauung, Alter sowie sexuelle Orientierung bleiben Benachteiligungen aufgrund des Geschlechts der zentrale Arbeitsbereich der Beauftragten. Probleme der Ungleichbehandlung werden aber nach Möglichkeit im Vorfeld gelöst. Nichtsdestotrotz kommt der Kommission eine zentrale Funktion als „Rute im Fenster" zu, die sowohl die Rolle des Arbeitskreises für Gleichbehandlungsfragen stärkt als auch die Prozessabläufe in Personalverfahren beeinflusst.

Welche Agenden in der Praxis verfolgt werden, für welche Belange sie sich engagieren, bleibt aber immer ein Stück weit in der persönlichen Verantwortung. Dies umso mehr, als mit Ausnahme einzelner Ressorts (z.B. Justizministerium) kein konkretes Zeitbudget für die Tätigkeit vorgesehen ist. Wieweit eine eigens dafür reservierte Zeit ihre Arbeit unterstützen würde, wird von den Gleichbehandlungsbeauftragten kontrovers diskutiert. Einerseits wird argumentiert, dass ein festgelegtes Zeitbudget den Beauftragten eher schaden könnte, weil sie damit auch formell weniger in ihren sonstigen Funktionen involviert wären und ein negativer Anreiz für die Bestellung von Gleichbehandlungsbeauftragten in der eigenen Abteilung bestünde. Andererseits kann ein fehlendes Zeitbudget für Gleichbehandlungsagenden dazu führen, dass diese Aufgaben weniger Aufmerksamkeit erhalten.

Das persönliche Engagement der Gleichbehandlungsbeauftragten ist nicht nur abhängig vom Zeitbudget und von ihrer Persönlichkeit, sondern auch von ihrer Position in der Organisation. Nach Ansicht der Gleichbehandlungsbeauftragten steigt ihr Handlungsspielraum mit der Position: Je höher ihre Funktion ist, desto mehr Gewicht wird ihnen zugesprochen und desto eigenständiger können sie Tätigkeiten und Zeit für Gleichbehandlungsagenden planen.

Hinsichtlich Interessensvertretung und Positionierung innerhalb der Organisation nehmen Gleichbehandlungsbeauftragte eine Doppelfunktion ein. Als Unterstützung der Ressortleitung, von der die Gleichbehandlungsbeauftragten auch bestellt werden, vertreten sie die Interessen der Arbeitgeber und sind teilweise auch in strategische Funktionen der Personalpolitik eingebunden. Mit dieser Zuordnung werden Gleichbehandlung und Frauenförderung in der Ressortleitung verankert. Als VertreterInnen benachteiligter Gruppen übernehmen sie aber auch ArbeitnehmerInneninteressen in Personalverfahren, in einzelnen Fällen in Koalition mit der Arbeitnehmervertretung. Dies führt in der Praxis mitunter zu Konflikten und kann negative Konsequenzen für ihre eigene Karriere haben - auch wenn in den Frauenförderplänen festgehalten ist, dass den Gleichbehandlungsbeauftragten keine Nachteile aus 
ihrer Tätigkeit entstehen dürfen. In der Wahrnehmung anderer Bundesbediensteter werden Gleichbehandlungsbeauftragte weniger mit Arbeitgeberinteressen assoziiert, sondern eher als unabhängige Einrichtung wahrgenommen. Damit ist einerseits Unabhängigkeit verbunden, andererseits wird dies aber auch als Mangel beurteilt. Denn ohne Bündnispartner wie Arbeitgeber oder Arbeitnehmervertretung bleibt ihr Einflussbereich gering.

Von den Gleichbehandlungsbeauftragten werden weitere Punkte thematisiert, die ihre Einflussmöglichkeiten entscheidend begrenzen: So hat die Arbeitsgruppe für Gleichbehandlungsfragen zwar ein Vorschlagsrecht für die Formulierung des Frauenförderplans, aber dieser muss von der Ressortleitung genehmigt werden und wird in der Praxis laut Berichten der ExpertInnen mitunter stark beschnitten. Durch die temporäre Bestellung der Gleichbehandlungsbeauftragten ist eine große Abhängigkeit von der Ressortleitung gegeben. In der Praxis gibt es einige Frauen, die seit langem als Gleichbehandlungsbeauftragte fungieren, doch die Kontinuität der Beauftragten ist in den einzelnen Ressorts unterschiedlich. Im Erhebungszeitraum wurde beobachtet, dass ein großer Teil der Beauftragten nicht wiederbestellt wurde, was die Effektivität der Arbeitskreise wegen fehlender Kontinuität und Verlust von Know-how einschränkt. Auch die Durchschlagskraft der Gleichbehandlungskommission wird als begrenzt wahrgenommen, insbesondere was die Lösungsmöglichkeiten für die Betroffenen bei Personalverfahren oder sexueller Belästigung betrifft. Doch auch wenn die Chancen auf Aufhebung einer festgestellten Ungleichbehandlung in Personalverfahren von den Gleichbehandlungsbeauftragten als eher gering eingeschätzt werden, verändern sie Personalverfahrensprozesse und tragen damit wesentlich zu längerfristigen Veränderungen bei.

\subsubsection{Zwischenfazit: Trotz starker Institutionalisierung wenig Verbindlichkeit}

In Österreich ist die institutionelle Gleichstellungspolitik des Bundes stark formalisiert. Mit den gesetzlichen Regelungen des B-GlBG werden einheitliche Gleichstellungsinstitutionen durch Gleichbehandlungsbeauftragte und deren Vernetzung in ressortinternen Arbeitskreisen und in der interministeriellen Arbeitsgruppe für Gleichbehandlungsbeauftragte, die Gleichbehandlungskommission sowie Frauenförderpläne und die darüber eingerichtete Berichtspflicht in Form der Gleichbehandlungspläne festgelegt. Bemerkenswert ist die frühe Institutionalisierung von Gleichstellungspolitik im öffentlichen Dienst in Österreich: Bereits im Jahr 1981 wurde in einem Ministerratsbeschluss ein Frauenförderprogramm für den Bundesdienst geschaffen, das zentrale Aspekte des Gleichbehandlungsgesetzes beinhaltete. Mit der Verankerung von Gleichbehandlungsbeauftragten und Frauenförderplänen wurden 
Zuständigkeiten sowie Gleichstellungsschwerpunkte und mit den initiierten Evaluationen die Basis für eine kontinuierliche Reflexion der Gleichstellung geschaffen.

Durch die gesetzliche Verankerung weisen die Vorgaben zum Aufbau von Gleichstellungsstrukturen eine hohe Verbindlichkeit für den gesamten Bundesdienst auf, sind aber hinsichtlich ihrer inhaltlichen Ausgestaltung wenig ausdifferenziert. Die inhaltliche Konkretisierung liegt in der Verantwortung der Ministerien, indem gleichstellungspolitische Schwerpunktsetzungen und konkrete Maßnahmen in Form von Verordnungen, den Frauenförderplänen, festgelegt werden. Der Vergleich der Frauenförderpläne und der Informationen im Gleichbehandlungsbericht des Bundes zeigt eine große Vielfalt von Schwerpunkten und eine sehr unterschiedliche Ausdifferenziertheit der Maßnahmen.

Das Sozialministerium und das Finanzministerium weisen sehr ambitionierte Frauenförderpläne auf, die im Vergleich zu anderen Ressorts differenzierte Regelungen für die Integration von Eltern in Karenz und Teilzeitbeschäftigung sowie für die Einbindung von Männern in Vereinbarkeitsmaßnahmen aufweisen und Gleichstellung als Teil der Personalstrategie festlegen. Sie räumen den Gleichbehandlungsbeauftragten auch die Möglichkeit zur Teilnahme an Personalverfahren ein. Die Unterschiede in den Frauenförderplänen, die als Ausdruck eines unterschiedlichen Engagements für Gleichstellungspolitik interpretiert werden können, sind auf mehrere Faktoren zurückzuführen: Zum einen hängen sie mit dem Gegenstand der Ressorts und seiner Nähe zu Gleichstellungspolitik zusammen. In den sozialkulturellen Bereichen wie Arbeit oder Wissenschaft und Bildung, die Kernbereiche der Gleichstellungspolitik ansprechen, sind sowohl die Repräsentanz als auch das Engagement für betriebliche Gleichstellungspolitik traditionell stark. Zum anderen hängt dies aber auch mit dem persönlichen Engagement der Akteurinnen zusammen. Es besteht ein großer Gestaltungsspielraum für den Einflussbereich der Gleichbehandlungsbeauftragten, der im Finanzministerium trotz des an sich männlich dominierten Bereichs zu einer ausdifferenzierten Gleichstellungspolitik geführt hat.

Die Gleichbehandlungsbeauftragten bilden nach wie vor das Rückgrat der Gleichstellungspolitik, indem sie als Personen Maßnahmen zur Gleichstellungspolitik einfordern, entwickeln, umsetzen und auch kontrollieren. Dies hat sich seit Implementierung des Frauenförderprogramms nicht verändert auch nicht durch die gesetzliche Verankerung des Gender MainstreamingAnsatzes, der Gleichstellungspolitik als Top-down-Strategie und in der Umsetzungsverantwortung aller AkteurInnen definiert. Ihr Einfluss im Bundesdienst weist Parallelen zur Tätigkeit der Gleichbehandlungsbeauftragten an Universitäten oder in der Arbeitsmarktpolitik auf. Sie prägen die Personalpolitik durch Förderung von Transparenz und Objektivität von Verfahren und sind entscheidend in die Ausgestaltung von Frauenfördermaßnahmen invol- 
viert (Wroblewski/Leitner 2011; Leitner 2007). Schon im Frauenförderprogramm des Bundesdienstes wurde den sog. Kontaktfrauen eine zentrale Rolle für die Sensibilisierung für Gleichstellungsfragen und die Unterstützung von Gleichstellung zugeschrieben, ihre Verankerung in der Organisation wie auch ihr Aufgabengebiet wurden jedoch als schwach ausgebaut und unklar beschrieben (Kreisky/Walther 1990). Mit der Implementierung des Gleichbehandlungsgesetzes wurden grundlegenden Rahmenrichtlinien für ihr Aufgabengebiet definiert und Gleichbehandlungsbeauftragte als Unterstützung der Ressortleitung installiert.

Gleichbehandlungsbeauftragte sind nicht nur für Frauenförderungs- und Gleichbehandlungsagenden im engen Sinne zuständig, sondern mit dieser Thematik in die gesamte Personalpolitik involviert, freilich kaum als Entscheidungsträgerinnen, sondern in ihrer Rolle als beratende Unterstützung für Entscheidungsgremien. So können sie als Arbeitsgruppe für Gleichbehandlungsfragen einen Vorschlag für den Frauenförderplan erstellen, die Entscheidung über die darin enthaltenen Ziele und Schwerpunkte bleibt aber bei der Ressortleitung. In der Organisation werden sie aber oft als „Feministinnen" wahrgenommen, indem sie personalpolitische Strategien und Verfahren kritisch reflektieren, damit auch häufig in Opposition zu Bereichsverantwortlichen und Leitungspersonen stehen. Für die Gleichbehandlungsbeauftragten selbst bedeutet dies, dass sie mitunter primär als Kritikerinnen des Systems wahrgenommen und ihre fachlichen Kompetenzen nicht honoriert werden.

Die Situation der Gleichbehandlungsbeauftragten im österreichischen Bundesdienst unterscheidet sich sowohl hinsichtlich ihrer Bestellung als auch der nicht geregelten Zeitbudgets für Gleichbehandlungsagenden von der Schweiz und Deutschland: Mit der Zuständigkeit für Gleichbehandlungsagenden als Nebentätigkeit ohne fixes Zeitbudget sind ihre Möglichkeiten wesentlich von ihrer beruflichen Position abhängig. Durch die zeitlich befristete Bestellung ist eine größere Abhängigkeit von der Ressortleitung gegeben als bei der Wahl durch die Belegschaft (Deutschland) oder unbefristete Bestellung (Schweiz). Ihre Doppelfunktion als Vertretung der Ressortleitung und als Interessensvertretung von benachteiligten Gruppen beinhaltet ein Konfliktpotenzial, das ihrer Position als eigenständige Vertretung von Fraueninteressen förderlich sein kann, die Gleichstellungsbeauftragten aber mitunter in ein „Fraueneck“ verweist, in dem sie wenig Mitwirkungskraft besitzen und schwerlich Bündnispartner für ihre Anliegen finden.

\subsection{Substanzielle Politik im österreichischen Bundesdienst}

Wie in den beiden anderen Ländern stellen auch im österreichischen Bundesdienst Maßnahmen zur Vereinbarkeit von Beruf und Familie eine, wenn nicht 
die zentrale Säule der Gleichstellungspolitik dar. Ein zweiter Schwerpunkt liegt bei Maßnahmen zur Karriereförderung von Frauen, die durch das Frauenfördergebot und die Frauenquote unterstützt werden. Darüber hinaus sind gesetzliche Maßnahmen zur Gleichbehandlung gegeben, wie die sprachliche Gleichbehandlung oder die Vermeidung von sexueller Diskriminierung, auf die hier aber nicht näher eingegangen wird.

\subsubsection{Maßnahmenvielfalt zur Vereinbarkeit von Familie und Beruf}

Maßnahmen zur Vereinbarkeit von Beruf und Familie sind primär über das Beamtendienstrecht bzw. Vertragsbedienstetengesetz geregelt. Ähnlich den gesetzlichen Bestimmungen für die Privatwirtschaft besteht auch im Bundesdienst

- ein Beschäftigungsverbot für werdende Mütter jeweils acht Wochen vor und nach der Geburt;

- ein Anspruch auf Karenz sowohl für Mütter als auch Väter bis zum Ablauf des zweiten Lebensjahres des Kindes;

- die Wahl zwischen fünf Modellen des Kinderbetreuungsgeldes mit unterschiedlicher Bezugsdauer zwischen zwölf und 36 Monaten, wobei jeweils eine Beteiligung beider Elternteile vorgesehen ist;

- ein Anspruch auf Herabsetzung der wöchentlichen Arbeitszeit bis zum Schuleintritt der Kinder; und

- die Möglichkeit eines Karenzurlaubs (ohne Bezüge) zur Pflege eines im selben Haushalt lebenden behinderten Kindes (bis zu dessen Vollendung des 40. Lebensjahrs) sowie zur Pflege eines pflegebedürftigen Angehörigen.

Bezüglich der gesetzlichen Bestimmungen unterscheidet sich der öffentliche Dienst von der Privatwirtschaft vor allem durch den sog. „Papamonat“, der Vätern die Möglichkeit einer frühzeitlichen Karenz von bis zu vier Wochen, auch bereits während des Beschäftigungsverbots der Mutter, einräumt ( $\S 75 \mathrm{~d}$ BDG, § 29o VBG). Die Forderung nach einem Vaterschaftsurlaub konnte damit bislang nur für den öffentlichen Dienst umgesetzt werden.

Im B-GlBG kommt der Vereinbarkeit von Beruf und Familie wenig Bedeutung zu und wird lediglich über ein Verbot der unmittelbaren Diskriminierung von Personen aufgrund von Schwangerschaft, Beschäftigungsverbot unmittelbar vor oder nach der Geburt eines Kindes, Berufsunterbrechung, Teilbeschäftigung oder Herabsetzung der Wochendienstzeit angesprochen. Alle Bestimmungen zur Gleichbehandlung und Frauenförderung beziehen sich auf die Kategorie Geschlecht bzw. sonstige Ungleichheitsdimensionen wie Alter, ethnische Zugehörigkeit etc. 
In den Frauenförderplänen sind über die gesetzlichen Bestimmungen hinausgehende Regelungen zur Förderung von Eltern und WiedereinsteigerInnen geregelt. Die Schaffung von Rahmenbedingungen für die Vereinbarkeit von Familie und Beruf sowie die Förderung der Akzeptanz der Inanspruchnahme von Karenz und Teilzeit im Ressort werden in allen Frauenförderplänen der Ministerien als grundlegende Ziele definiert. Weitere Schwerpunktsetzungen zur Vereinbarkeit von Familie und Beruf in den Frauenförderplänen (respektive in jenen des Sozialministeriums und des Finanzministeriums) betreffen Maßnahmen zur Erleichterung der Rückkehr nach der Karenz, für eine flexible Arbeitszeitgestaltung, zur Einbeziehung der Väter bei Karenz und Teilzeit sowie zur Unterstützung von Kinderbetreuung.

Insgesamt besteht eine Vielzahl von Maßnahmen, die in den Frauenförderplänen zur Unterstützung der Vereinbarkeit von Familie und Beruf angeführt sind. Wie die Daten und Einschätzungen der ExpertInnen darlegen, sind diese Maßnahmen in unterschiedlicher Weise wirksam.

\section{Maßnahmen zur Erleichterung der Rückkehr nach der Karenz}

Neben der gesetzlichen Verankerung eines Kündigungsverbots nach der Karenz und der Rückkehrmöglichkeit auf den früheren Arbeitsplatz besteht eine Reihe von Maßnahmen, die den Wiedereinstieg nach der Karenz erleichtern sollen:

- Informationsangebote für Eltern über die Karenz und die Verwendung nach der Rückkehr sind in allen Frauenförderplänen der Ressorts vorgesehen.

- Spätestens vier Wochen vor dem Wiedereinstieg soll ein Gespräch über die künftige Verwendung, über Möglichkeiten eines gleitenden Wiedereinstiegs sowie einer flexiblen Arbeitszeitgestaltung erfolgen. Im Frauenförderplan des Sozialministeriums wird darauf hingewiesen, dass in MitarbeiterInnengesprächen die Übernahme von Betreuungspflichten durch Männer zu unterstützen ist.

- Im Frauenförderplan des Finanzministeriums wird die Möglichkeit einer freiwilligen Teilnahme von karenzierten Bediensteten an Dienstbesprechungen sowie die Übermittelung von Unterlagen und Informationen eingeräumt.

- Karenzierte MitarbeiterInnen sind über Weiterbildungsmöglichkeiten zu informieren, die zeitlich nach der Karenz liegen. Der Frauenförderplan des Finanzministeriums sieht vor, dass MitarbeiterInnen auf Ersuchen auch früher über interne und externe Weiterbildungsmöglichkeiten sowie über andere Angelegenheiten wie Organisationsänderungen, Änderungen im Tätigkeitsbereich oder Funktionsausschreibungen in Kenntnis gesetzt werden müssen. 
- Einige Ressorts sehen eine geeignete Nachschulung von wieder eintretenden karenzierten Bediensteten vor. Die vorrangige Zulassung von Karenzierten zu Weiterbildungsmaßnahmen ist im Frauenförderplan des Sozialministeriums vorgesehen, darüber hinaus soll für Wiedereinsteigerinnen auch Coaching und Einzelsupervision angeboten werden.

Die Rückkehrmöglichkeiten nach einer Karenz sind im Bundesdienst nach Einschätzung der ExpertInnen nicht nur gesetzlich verankert, sondern auch in der Praxis gegeben. Im Gegensatz zur Privatwirtschaft können Eltern nach Berufsunterbrechungen gewöhnlich damit rechnen, an ihren früheren Arbeitsplatz zurückzukehren. Als wesentliche Voraussetzung für den problemlosen Wiedereinstieg wird der Kontakt zu den Karenzierten angeführt. Dieser wird in den Interviews mit unterschiedlichen Aktivitäten sowohl auf informeller Ebene (z.B. Elterncafes) wie auch auf formeller Ebene (z.B. Dienstbesprechungen) illustriert. Dabei wird auch erwähnt, dass es möglich ist und in Einzelfällen vorkommt, sich während der Karenz erfolgreich für höhere Positionen zu bewerben.

Flexible Arbeitszeitgestaltung und Telearbeit

Grundlage dafür sind die dienstrechtlichen Regelungen sowie spezifischen Fördermaßnahmen für flexible Arbeitszeitgestaltung. Hinsichtlich der Möglichkeit zur Reduktion der Arbeitsstunden besteht ein Unterschied zwischen Beamten und Vertragsbediensteten, indem Vertragsbedienstete die Zahl der Arbeitsstunden mit dem Dienstgeber grundsätzlich frei vereinbaren können. Für BeamtInnen ist hingegen die Herabsetzung der Wochendienstzeit nach Beendigung des Bezuges von Kinderbetreuungsgeld auf höchstens 50\% der Vollbeschäftigung möglich (§50b BDG). Darüber hinaus sind ergänzende Bestimmungen in den Frauenförderplänen enthalten:

Für einen gleitenden Wiedereinstieg kann ein zweites, gesondertes Beschäftigungsverhältnis auf privatrechtlicher Basis für eine geringfügige Beschäftigung bzw. die Beschäftigung über der Geringfügigkeitsgrenze für maximal 13 Wochen abgeschlossen werden.

In einzelnen Frauenförderplänen, u.a. des Sozialministeriums, ist festgelegt, dass bei einem Umstieg auf Teilzeitbeschäftigung auf die entsprechende Reduzierung der Aufgabenbereiche zu achten ist. Im Zusammenhang mit Überstunden und Mehrarbeit wird darauf hingewiesen, dass Vorgesetzte dafür Sorge zu tragen hätten, dass die Aufgaben ihrer MitarbeiterInnen in der Regel in der Normalarbeitszeit zu bewältigen sind.

Auf die vorrangige Berücksichtigung von betreuungspflichtigen Personen bei flexiblen innovativen Arbeitsformen, insbesondere Telearbeit, wird in den Frauenförderplänen des Finanzministeriums und Sozialministeriums ausdrücklich hingewiesen. 
Im Bundesdienst wurde Teilzeitarbeit bis in die 1990er Jahre hinein wenig gefördert. Erst im Jahr 1979 wurde auch BeamtInnen die Möglichkeit eingeräumt, ihre Arbeitszeit auf bis zu 50\% ihrer Wochenarbeitszeit zu reduzieren (BDG 1979, § 50b). Während das Beamtendienstrecht keine Teilzeit unter 20 Wochenstunden zulässt, ist das Ausmaß der Arbeitszeit bei Vertragsbediensteten frei vereinbar. Weiterhin ist die Teilzeitquote der BeamtInnen mit $8 \%$ deutlich unter jener von Vertragsbediensteten $(30 \%)$. Wie in Abschnitt 6.2 angeführt, ist Teilzeit im Bundesdienst weniger stark verbreitet als in der Privatwirtschaft, aber auch hier gibt es eine steigende Tendenz.

Telearbeit wurde im Bundesdienst im Jahr 1999 zunächst als Pilotprojekt eingeführt. Seine Evaluierung zeigte eine hohe Motivation der Teilnehmenden sowie einen Nettonutzen für den Dienstgeber (Doppel et al. 2004). Die Möglichkeit zur Telearbeit wurde im Personalrecht verankert; es liegen aber keine Daten über das Ausmaß der Nutzung von Telearbeit vor. Nach Einschätzung der ExpertInnen wird Telearbeit im Verwaltungsdienst stark genutzt, insbesondere von Personen mit Betreuungspflichten. Dies werde aber in den Ressorts unterschiedlich gehandhabt.

\section{Einbeziehung von Männern bei Karenz und Väterteilzeit}

Die Einbeziehung der Väter erfolgt zum einen dadurch, dass viele Vereinbarkeitsmaßnahmen explizit an Frauen und Männer adressiert werden. Darüber hinaus wird eine Erhöhung der Akzeptanz von Väterkarenz und Väterteilzeit in den Frauenförderplänen des Sozialministeriums und des Finanzministeriums angeführt.

Die Versuche Väter zu Karenz und Väterteilzeit zu bewegen, werden von den ExpertInnen allerdings als wenig erfolgreich beurteilt, und Väter in Karenz oder Teilzeit werden auch im Bundesdienst nach wie vor als Ausnahme wahrgenommen. Dies zeigt sich ebenfalls in den dazu verfügbaren Daten (Pressemeldung der Frauenministerin; vgl. http://oe1.orf.at/artikel/322596; Zugriff: 14.02.2015): Zwischen 2011 und 2012 hat nur etwa jeder neunte Vater die Möglichkeit der frühkindlichen Karenz genutzt. Der Anteil der Väter in Karenz im Bundesdienst liegt mit 12\% zwar deutlich über ihrem Anteil in der Privatwirtschaft (4\%), betrifft damit aber nur ein Achtel der Väter.

\section{Unterstützung von Kinderbetreuung}

Auf die Sicherung der Kinderbetreuung wird in allen Frauenförderplänen eingegangen. Konkret wird meistens eine Überprüfung des Bedarfs an Kinderbetreuungseinrichtungen in der Nähe der jeweiligen Dienststelle angeführt. Darüber hinaus werden institutionelle Kinderbetreuungsangebote über den Betriebskinderkarten für Bundesbedienstete mit einem hohen Qualitäts- 
niveau (bezüglich Betreuungsverhältnis und pädagogischer Angebote) bereitgestellt und auch ausgebaut.

Nach Einschätzung einzelner ExpertInnen wird der Unterstützung von Kinderbetreuung aber zu wenig Aufmerksamkeit und Bedeutung eingeräumt. Die Organisation des Alltags mit Kindern ist ohne die Schaffung institutioneller Infrastrukturen, die sowohl pädagogischen Bedürfnissen der Kinder wie auch organisatorischen und zeitlichen Entlastungsbedürfnissen der Eltern Rechnung tragen, schwer zu bewältigen. In diesem Kontext erscheint die institutionelle Gewährleistung von entsprechenden Strukturen, welche eine Vollzeitbeschäftigung von Frauen ermöglichen, als besonders wichtig, sie wird auch im Bundesdienst in den Interviews als nicht ausreichend beurteilt.

\subsubsection{Versuche zur Vermeidung von Karriererisiken durch Vereinbarkeitsmaßnahmen}

Gerade im Bereich der Vereinbarkeitspolitiken sind die gleichstellungspolitischen Maßnahmen sehr ausdifferenziert. Dies gilt sowohl für die personalrechtlichen Regelungen zu Karenz, Teilzeit- oder Telearbeit als auch für darüber hinausgehende Maßnahmen. In den Frauenförderplänen sind zum einen proaktive Verfahren zur Integration von Karenzierten und Teilzeitbeschäftigten sowie Maßnahmen verankert. Damit werden die Risiken eines Karriereknicks oder des Arbeitsplatzverlustes durch lange Berufsunterbrechungen, die in der Privatwirtschaft verstärkt gegeben sind, eingeschränkt. Zum anderen werden Maßnahmen implementiert, um mehr Männer in Betreuungsaufgaben einzubeziehen - auch wenn sich dies als ein langwieriger Prozess mit bislang geringen Erfolgen erweist. Die Verbindlichkeit der Regelungen ist dadurch eingeschränkt, dass diese in den Frauenförderplänen als Kann-Bestimmungen formuliert sind, d.h. kein grundsätzlicher Anspruch auf Teilzeit oder einen flexiblen Arbeitsort besteht bzw. die Gewährung von der organisatorischen Umsetzung abhängig ist.

Die Frauenförderpläne des Finanzministeriums wie auch des Sozialministeriums enthalten weitreichende Maßnahmen zur Vereinbarkeit von Beruf und Familie, die über Regelungen der anderen Ressorts hinausgehen: Klare Richtlinien zur Information von Karenzierten über zukünftige Verwendung und Weiterbildungsmöglichkeiten sollen den erfolgreichen Wiedereinstieg nach der Karenz erleichtern. Karenzierten Bediensteten wird die Teilnahmemöglichkeit an Besprechungen formell eingeräumt. Eine weitere Besonderheit, die das Finanz- und das Sozialministerium mit dem Innen- und dem Wirtschaftsministerium teilen, ist die spezifische Förderung der Akzeptanz von Väterkarenz. Hingegen zeigen sich das Finanz- und Sozialministerium bei der Förderung flexibler Arbeitszeitgestaltung im Vergleich zu den anderen Ressorts eher zurückhaltend. 
In den Gesprächen mit den AkteurInnen der Ministerien zeigt sich ein hohes Problembewusstsein für die Zweischneidigkeit der Vereinbarkeitsförderung durch Teilzeit- und Telearbeit sowie Berufsunterbrechungen. Die Förderung einer vorübergehenden Arbeitszeitreduktion ermögliche zwar unter den gegebenen Bedingungen der Arbeitsteilung der Geschlechter und des Angebots an Betreuungseinrichtungen mitunter überhaupt eine Erwerbstätigkeit, aber die Wirkung auf die Karrierechancen und Existenzsicherung sei negativ. Damit sehen vor allem Gleichbehandlungsbeauftragte die Tendenz zu zunehmender Teilzeiterwerbstätigkeit eher skeptisch. Die Möglichkeiten zur Arbeitszeitreduktion seien zwar in Phasen mit kleinen Kindern wichtig, sollten aber nicht verstärkt gefördert werden. Es sei jedenfalls keine Fördermaßnahme, durch die die Frauenproblematik allein gelöst werden kann. Insofern wird auch davor gewarnt, diese Vereinbarkeitsmaßnahmen in den Mittelpunkt der Frauenförderung zu stellen.

Neben den Karriererisiken wird auch auf die individuellen Belastungen durch Teilzeit hingewiesen. Dies wird besonders deutlich, wenn AbteilungsleiterInnen stolz berichten, dass Teilzeitbeschäftigte das Arbeitspensum von Vollzeitbeschäftigten erfüllen (vgl. auch Mitsching-Viertel 2012). Zugleich werde dadurch, dass eine Arbeitszeitreduktion während der Familienphase primär von Frauen in Anspruch genommen wird, die private Arbeitsteilung in Richtung der weiblichen Verantwortung für Betreuungs- und Haushaltsarbeit verstärkt.

Ähnliche Risiken werden auch bezüglich der Telearbeit gesehen. Die Akzeptanz von Teilzeit wurde in den Interviews unterschiedlich wahrgenommen. Mehr noch als Teilzeitarbeit wird die unzureichende gesetzliche Reglung bzw. der mangelnde rechtliche Anspruch darauf thematisiert.

Entsprechend den verschiedenen arbeitsrechtlichen Bestimmungen unterscheiden sich die Möglichkeiten der Teilzeitarbeit bei Beamten und Vertragsbediensteten. Teilzeit ist nunmehr auch für Beamte möglich, aber auf mindestens eine 50\%-Beschäftigung begrenzt; nur während des Karenzgeldbezugs ist auch für Beamte eine geringere Arbeitszeit möglich. Dies verweist auf das historische Verständnis von Beamtentum, das eine ausschließliche und lebenslange Dienstpflicht vorsieht. So ist auch in den Bestimmungen zur Karenzzeit festgelegt, dass den Beamten auf Antrag ein Urlaub unter Wegfall der Bezüge (Karenzurlaub) gewährt werden kann, sofern nicht zwingende dienstliche Gründe dagegen sprechen ( $\$ 75$ BDG). Die Verbindlichkeit ist aber insgesamt bei den personalrechtlichen Regelungen höher als bei den Maßnahmen der Frauenförderpläne. Damit bleiben die Maßnahmen, die die negativen Folgen von langen Berufsunterbrechungen und Teilzeitbeschäftigung abfedern sollen, wesentlich von den beteiligten AkteurInnen abhängig. 


\subsubsection{Karriereförderung über Weiterbildung und Prozessregelungen bei Personalverfahren}

Mit Maßnahmen zur Karriereförderung wird explizit Frauenförderung betrieben, d.h. von dem sonst geltenden Grundsatz der Gleichbehandlung, der sich auch in den österreichischen Bezeichnungen Gleichbehandlungsgesetz oder Gleichbehandlungsbeauftragte niederschlägt, abgegangen. Um die Unterrepräsentanz von Frauen an der Gesamtzahl der Beschäftigten und der Funktionen im Bundesdienst zu beseitigen, bedarf es der Frauenförderung. Mit dem Frauenfördergebot ( $\$ 11 \mathrm{~B}-\mathrm{GlBG})$ sind die VertreterInnen des Bundesdienstes verpflichtet, der Unterrepräsentanz von Frauen an der Gesamtzahl der Beschäftigten nach Besoldungsgruppen, Funktionsgruppen und Verwendungen entgegenzuwirken. Es gilt, mittels Frauenfördermaßnahmen eine 50\%Quote für Frauen in allen Beschäftigungsbereichen zu erreichen. Handlungsbedarf ist damit vor allem in den oberen Hierarchiestufen und in den traditionellen Männerbastionen gegeben. Denn wie in Abschnitt 6.2 dargestellt, betrifft die Unterrepräsentanz primär Führungspositionen sowie den stark männlich dominierten Exekutiv- und Militärdienst. Die Förderung von Frauen in höheren Positionen sollte aber auch zur Verbesserung aller im Bundesdienst beschäftigten Frauen beitragen.

Im Bundesgleichbehandlungsgesetz sind eine vorrangige Aufnahme in den Bundesdienst, Vorrang beim beruflichen Aufstieg und Vorrang bei der Aus- und Weiterbildung festgelegt ( $\$ 11 \mathrm{~b}-\mathrm{c}$ B-GlBG). Darüber hinaus sind in den Frauenförderplänen der Ressorts Maßnahmen zu spezifizieren, die die Unterrepräsentanz von Frauen beseitigen. Zudem sind jeweils für zwei Jahre verbindliche Vorgaben zur Erhöhung des Frauenanteils festzulegen $(\S 11 \mathrm{a}$ B-GlBG).

In den Frauenförderplänen der Ressorts sind folgende Maßnahmen zur Karriereförderung von Frauen angeführt:

- Zielwerte für die Beseitigung der Unterrepräsentanz von Frauen: Wie konkret Frauenquoten als Zielwerte für die Beseitigung von Unterrepräsentation definiert werden, wird unterschiedlich gehandhabt. Beispielsweise werden in den Frauenförderplänen des Finanz- und des Sozialministeriums konkrete Zielwerte für unterschiedliche Beschäftigungsbereiche angegeben, in anderen Frauenförderplänen hingegen lediglich allgemeine Richtwerte für Steigerungen.

- Festschreibungen in Bezug auf Stellenausschreibungen und Bewerbungsverfahren, die auf eine größere Transparenz der Verfahren, die Vermeidung von frauendiskriminierenden Fragestellungen oder auch auf die Motivation von Bewerberinnen zielen.

- Zugang zu Aus- und Weiterbildung: Für die Verbesserung der Karrierechancen nimmt die Aus- und Weiterbildung einen hohen Stellenwert ein. 
Dementsprechend werden vorrangige Zugangschancen für Frauen in der Aus- und Weiterbildung festgelegt, sollen Frauen spezifisch für die Teilnahme motiviert werden und wird die Teilnahme nach Geschlecht regelmäßig ausgewiesen.

- Frauenspezifische und gendergerechte Aus- und Weiterbildungen: Neben den allgemeinen Weiterbildungsmaßnahmen werden auch spezifische Qualifizierungen für Frauen in Form von Laufbahn- oder Karriereseminaren angeboten. Zusätzlich werden Bedingungen für gendergerechte Unterrichtsmaterialien oder Richtlinien für die Vortragenden in den Frauenförderplänen vorgegeben. Darüber hinaus führen die Frauenförderpläne des Sozial- und Finanzressorts Mentoringprogramme sowie die Ermöglichung von Coaching bzw. Supervision an.

In den rechtlichen Verordnungen wird also ein breites Angebot an Fördermaßnahmen für Frauen zur Erhöhung ihrer Karrierechancen im Bundesdienst angesprochen. Die Maßnahmen zur Karriereförderung von Frauen betreffen zum einen Qualifizierungsmaßnahmen, die Frauen befähigen, bestimmte Positionen zu bekleiden, zum anderen Vorgaben für Personalverfahren, um Auswahlprozesse transparent und nachvollziehbar zu gestalten und Frauen dabei nicht zu benachteiligen bzw., bei gleicher Qualifikation wie die Männer, bevorzugt zu behandeln.

Die Maßnahmen zielen auf zwei Schwerpunktgruppen. Einerseits geht es dabei um die Förderung von Karenzierten bzw. Teilzeitbeschäftigten, wobei Männer grundsätzlich einbezogen sind, Frauen aber aufgrund der unterschiedlichen Nutzung primär betroffen. Dabei wird also eine konkrete Verbindung zu Vereinbarkeitsregelungen hergestellt, weil es darum geht, die negativen Folgen von Vereinbarkeitsregelungen auf die Karriere auszugleichen. Andererseits sollen Frauen in Führungspositionen gebracht werden, es geht also um Elitenförderung in der Gleichstellungspolitik. Dies wird auch mit der Quotenvorgabe, die eine Frauenförderung für Bereiche mit weniger als $50 \%$ weiblichen Beschäftigten vorsieht, unterstützt. Denn die geringere Repräsentanz bezieht sich vor allem auf die höheren Tätigkeitsbereiche. Qualifizierungsmaßnahmen, die niedrig oder mittel qualifizierte Frauen explizit berücksichtigen, bleiben die Ausnahme; sie werden lediglich im Frauenförderplan des BMASK angesprochen.

Die Verbindlichkeit der für Karriereförderung verfolgten Maßnahmen erweist sich aber entsprechend der Formulierungen in den Frauenförderplänen als gering. Beispielsweise sollen Frauen über Bildungsangebote informiert, sollen für Frauen Plätze reserviert oder Frauen für Weiterbildungen motiviert werden. Für die Besetzung von Führungspositionen werden Prozessregelungen vereinbart wie z.B. Regelungen für Ausschreibungen. Über Festlegungen für MitarbeiterInnengespräche (z.B. Ansprechen von flexibler Arbeitszeitgestaltung auch bei Männern) sollen Entwicklungsmöglichkeiten 
aufgezeigt werden. Es geht vielfach um Strategien, die die Karrieremotivation von Frauen heben sollen.

\subsubsection{Frauenquoten als Schlüsselinstrument der Gleichstellung}

Diese Verankerung von Frauenförderung bei Rekrutierung, Aufstieg und Weiterbildung wäre nach Angaben der GesprächspartnerInnen ohne die gesetzliche Frauenquote nicht durchsetzbar. Die Quotenvorgaben zur Vermeidung der Unterrepräsentanz von Frauen und die damit verbundenen kontinuierlichen Berichte über den Frauenanteil werden sowohl für die Karriereförderung als auch für Maßnahmen zur Vereinbarkeit von Familie und Beruf als essenziell für deren Wirksamkeit in der Praxis angesehen.

Die Frauenquote ist im Bundes-Gleichbehandlungsgesetz nicht als grundsätzliche Entscheidungsregel, sondern als „Vorrangregel“ formuliert, die eine Frauenförderung bedingt, solange sie in der jeweiligen Funktion unterrepräsentiert sind (Jarosch 2001). Durch Verknüpfung der Regel mit der Unterrepräsentanz ist ein enger Konnex der Frauenförderung zur strukturellen Diskriminierung gegeben. Frauenquoten im Bundesdienst werden von den ExpertInnen als weitgehend akzeptiert eingeschätzt. Entscheidend unterstützt wird die Quotenvorgabe durch die regelmäßige Berichterstattung über die Präsenz von Frauen in den unterschiedlichen Verwendungsgruppen und Funktionen. Mit dem Monitoring durch den Gleichbehandlungsbericht wird das Bewusstsein über strukturelle Diskriminierung erhöht, indem diese sichtbar gemacht wird. Es erlaubt grundsätzlich auch einen Vergleich zwischen den Ressorts zur Hervorhebung besonders erfolgreicher oder weniger erfolgreicher Ministerien.

Wie von den ExpertInnen betont wird, führen die Quotenregelungen kaum dazu, dass in Rekrutierungsverfahren bestimmte Frauen durchgesetzt werden können, wenn sie gegenüber Männern gleichwertige Qualifikationen aufweisen, da gleiche oder auch bessere Qualifikationen schwer nachweisbar sind. Doch mit der Veröffentlichung dieser statistischen Berichte zum Geschlechteranteil entsteht für die Ressorts ein Rechtfertigungsdruck. Dies unterstützt Frauenfördermaßnahmen und führt zu einer Veränderung von Personalverfahren, indem diese professionalisiert und transparenter gemacht werden.

Wie Hackl und Steger-Mauerhofer (2012) zeigen, ist ein Anstieg der Frauen in den Führungspositionen innerhalb der letzten 20 Jahre erkennbar. Die Tatsache, dass der Frauenanteil in Führungspositionen im Bundesdienst stärker gestiegen ist als in anderen Berufsfeldern von AkademikerInnen, wie z.B. bei RechtsanwältInnen, spricht aus ihrer Sicht für die Wirksamkeit des Bundes-Gleichbehandlungsgesetzes bzw. der Frauenquoten. Seine Wirksamkeit für eine Gleichstellung von Frauen und Männern wird von ihnen aber mit 
dem Argument hinterfragt, dass gerade die Leitungsfunktionen, die Frauen erreichen konnten - die Sektionsleitungen -, durch eine Expansion der Ministerbüros, durch Einrichtung von Generalsekretariaten und die Befristung der Sektionsleitungen auf fünf Jahre eine Entmachtung erfahren haben. Entsprechend den Frauenanteilen von Verwendungs- bzw. Besoldungsgruppen wird aber auch hier sichtbar, dass mit diesen Maßnahmen zwar der Frauenanteil in den höherwertigen Verwendungsgruppen erhöht werden konnte, aber Top-Positionen, nämlich wichtige Sektionsleitungen oder auch Ministerbüros, primär Männern vorbehalten bleiben. Dies wird von den GesprächspartnerInnen auch darauf zurückgeführt, dass in diesen Bereichen Rekrutierungsverfahren weniger transparent sind, es sich dabei oft um politische Entscheidungen handelt oder, wie dies im Bundesdienst umschrieben wird, „Personen des Vertrauens" bestellt werden.

Die Quotenregelung, die eine Bevorzugung von Frauen in Personalverfahren bei Unterrepräsentanz vorsieht, weist aufgrund der Voraussetzung, dass Frauen bei gleicher Qualifikation vorzuziehen sind, aber eine eingeschränkte Verbindlichkeit auf. Denn oftmals mangelt es an der Beweisbarkeit der gleichen Qualifikation. Dennoch kommt der Quotenregelung eine zentrale Funktion für die Umsetzung von Gleichstellung zu. Denn mit der Quotenregelung und der Forderung nach Präsenz von Frauen in den Gremien werden die Geschlechterverhältnisse durchsichtiger.

\subsection{Fazit: Vorreiterrolle mit ungenutzten Potenzialen}

Im österreichischen Bundesdienst ist es nicht zuletzt durch die frühen und engagierten Schritte der Frauenförderung in den 1980er Jahren gelungen, Gleichstellungsmaßnahmen zu implementieren, die dem Bundesdienst eine Vorreiterfunktion gegenüber der Privatwirtschaft einräumen.

Vereinbarkeitsmaßnahmen werden auch im Bundesdienst als Voraussetzung für die Erwerbsintegration von Frauen gesehen und nehmen damit einen zentralen Stellenwert ein. Zusätzlich werden Maßnahmen implementiert, die die Inkonsistenzen im österreichischen Genderregime - das einerseits lange Berufsunterbrechungen und Teilzeitbeschäftigung von Frauen unterstützt, andererseits aber auch bei Frauen kontinuierliche Vollzeiterwerbskarrieren voraussetzt - abfedern. Dies betrifft Maßnahmen zur stärkeren Einbeziehung von Männern in die Kinderbetreuung (,Papamonat“, Erhöhung der Akzeptanz von Vätern in Vereinbarkeitsmaßnahmen), Informationspolitik für Eltern in Karenz, Förderung von Weiterbildung für WiedereinsteigerInnen, Teilzeitbeschäftigte etc.

Darüber hinaus bestehen zahlreiche Maßnahmen, die Frauenkarrieren unabhängig von Mutterschaft - fördern. Der ansonsten geltende Grundsatz 
der Gleichbehandlung von Frauen und Männern wird angesichts der Unterrepräsentanz von Frauen zugunsten eines Frauenfördergebots aufgehoben. Um ein ausgewogenes Geschlechterverhältnis, d.h. eine 50\%-Quote von Frauen in allen Berufspositionen zu erreichen, sind Frauen bei Weiterbildung (z.B. durch Vorrangregeln bei Weiterbildungsmaßnahmen, frauenspezifische Qualifizierungsmaßnahmen) und Personalverfahren (Prozessregelungen, Vorrangregeln bei Stellenbesetzungen) zu bevorzugen.

Mit dem Bundes-Gleichbehandlungsgesetz werden einheitliche Vorgaben für die Bestellung von Gleichbehandlungsbeauftragten, ihre Vernetzung innerhalb und zwischen den Ressorts, die Gleichbehandlungskommission und die Berichtspflicht festgelegt. Damit ist eine institutionelle Gleichstellungspolitik verankert, durch die eine Reflexion der Gleichstellung stattfinden kann. Die konkrete Ausgestaltung der Gleichstellungsziele und Schwerpunkte von Maßnahmen wie auch der Rechte und Pflichten von Gleichbehandlungsbeauftragten bleibt in der Verantwortung der Ressorts und erfolgt im Rang von Verordnungen. Durch Veröffentlichung der Frauenförderpläne und die Berichtspflicht über die Zielerreichung der Ressorts, die auf die Repräsentanz von Frauen in den unterschiedlichen Verwendungsgruppen und Positionen als zentralen Beobachtungsindikator abzielen, wird die Basis für ein Benchmarking gelegt, das über ,blaming and shaming“-Effekte die Anstrengungen und Verbindlichkeit für Gleichstellung stärken kann. Mit der Präsenz von Gleichbehandlungsbeauftragten in Kommissionen (beispielsweise für Personalverfahren) und den kontinuierlichen Berichten über die Gleichstellung von Frauen und Männern ist die Gleichstellungsthematik sichtbarer.

Frauenquoten und die Berichtspflicht über die Frauenquoten stellen den zentralen Hebel für die umgesetzte Gleichstellungspolitik dar. Durch die Vorgabe und Umsetzungstransparenz dieser Quoten werden Gleichstellungsmaßnahmen in die Diskussion gebracht und ernst genommen, besteht eine Motivation, Frauen in ihren Karrieren zu fördern. Das Netz von Gleichbehandlungsbeauftragten ist in dieses Konzept eingebunden, sieht sich oft zwar machtlos bei der Verhinderung individueller Benachteiligungen von Frauen, erhält aber durch die Quotenvorgaben eine Unterstützung für ihre Aufgaben und die Professionalisierung von Verwaltungsabläufen.

Die Beurteilung der Frage, wieweit der Bundesdienst die Chance seiner Vorreiterfunktion für die betriebliche Gleichstellungspolitik durch ein wirksames Steuerungsregime nutzt, fällt dennoch kritisch aus. Die gesetzlichen Regelungen des B-GlBG weisen zwar eine hohe Verbindlichkeit auf, sind aber wenig ausdifferenziert und werden damit von den Ministerien unterschiedlich interpretiert. Vereinbarkeitsmaßnahmen sind im Hinblick auf ihre Ausdifferenziertheit, Reichweite und Verpflichtung effektiver als Maßnahmen zur Karriereförderung. Denn während die personalrechtlichen Regelungen für Elternkarenz und Teilzeit eine hohe Verbindlichkeit aufweisen, sind die in den Frauenförderplänen verankerten Regelungen und Maßnahmen 
weniger bindend. Die explizite Verhinderung negativer Auswirkungen von Vereinbarkeitsmaßnahmen auf die Berufskarriere durch spezifische Regelungen bezieht sich nur auf Teilbereiche. Der steigende Anstieg der Teilzeitarbeit im öffentlichen Dienst nährt vielmehr die Befürchtung, dass auch in diesem Bereich das Nebeneinander von Beruf und Familie zunehmend über begrenzte Arbeit stattfindet, eine verstärkte Beteiligung der Männer hingegen kaum erreicht wird.

Entgegen dem Grundprinzip der staatlichen Verwaltung, die nur aufgrund der Gesetze ausgeübt werden kann $(\S 18 \mathrm{~B}-\mathrm{VG})$, ist Gleichstellungspolitik stark informell und vom Handeln einzelner Personen abhängig. Dies betrifft vor allem die Arbeit der Gleichbehandlungsbeauftragten, die gesetzlich wenig ausdifferenziert ist. Ihre Rolle wird - was ihre Bestellung betrifft wie auch ihre Rechte und Pflichten - wesentlich durch die Ressortleitung mitbestimmt. Ihre Gestaltungsmöglichkeiten werden aber auch von der Unterstützung anderer Personen, wie Personalverantwortlichen, Gremienvorsitzenden, Personalvertretung, wesentlich beeinflusst. Die Unterschiede der Gleichstellungspläne hinsichtlich Gleichstellungszielen, Schwerpunktsetzungen und Konkretheit von Vorgaben lassen auf unterschiedliche Akzeptanz von Gleichstellungsaktivitäten und Problembewusstsein für Handlungsbedarf schließen. Trotz Veröffentlichung der Frauenförderpläne und der Berichtspflicht zeigen sich wenig Tendenzen einer Annäherung zwischen den Ressorts, starke Differenzen bleiben bestehen. Dies spiegelt sich auch in der unterschiedlichen Frauenrepräsentanz der Ministerien wider: Insgesamt weist der österreichische Bundesdienst mit $41 \%$ einen im Vergleich zu Deutschland und der Schweiz hohen Frauenanteil auf, allerdings mit einer Bandbreite von $11 \%$ bis $61 \%$. Das Potenzial der Steuerungs- und Kontrollinstrumente für eine Standardisierung der Gleichstellungspolitik wird nicht genutzt.

Dementsprechend fällt die Beurteilung zur Entwicklung der Frauenbeschäftigung im Bundesdienst zweigeteilt aus: Zum einen wecken steigende Partizipationsanteile von Frauen - vor allem in den höchsten Verwendungsgruppen - die Hoffnung, dass mit der steigenden Qualifikation von Frauen leitende Stellen gleichermaßen erreichbar werden. Die unterschiedlichen Entwicklungen in den Berufsgruppen und Ressorts verweisen aber zum anderen darauf, dass die Fortschritte in Richtung Gleichstellung partiell erfolgen. Erklärungen durch verschiedene Aufgaben der Ressorts und ihre Nähe zu bzw. Tradition in Gleichstellungsfragen greifen dabei zu kurz.

Die vertikale Segregation, d.h. die geringere Präsenz von Frauen in Führungspositionen, die sich in den letzten Jahren verringert hat, aber einen weiterhin hohen Aufholbedarf aufweist, bestimmt den hohen Einkommensunterschied in den Verwaltungstätigkeiten mit. Trotz des starren, auf Seniorität ausgerichteten Besoldungssystems bewirken die unterschiedlichen Einstufungen von Frauen und Männern bei den Verwaltungstätigkeiten einen höhe- 
ren Gender Pay Gap als bei den unselbstständig Beschäftigten in der österreichischen Gesamtwirtschaft.

Der Frauenanteil in unterschiedlichen Positionen illustriert nicht nur die ungleichen Aufstiegschancen von Frauen und Männern, sondern ist auch ein Indikator dafür, wieweit sich die Kultur im Bundesdienst bezüglich der verfolgten Personalpolitik wie auch der externen Politik verändert hat. Frauenquoten für berufliche Positionen und Gremien bewirken daher auch mehr als „nur“ individuelle Frauen zu fördern: Sie lenken die Aufmerksamkeit auf Ungleichheiten zwischen Frauen und Männern, die statistisch eindeutig messbar sind, und unterstützen damit Maßnahmen und Personen, die solche Ungleichheiten zu beseitigen versuchen.

Selbstverständlich ist, wie dies die frühere Frauenministerin Johanna Dohnal immer wieder argumentiert hat, „Frau sein allein kein Programm“, und selbstverständlich arbeiten nicht alle Frauen, die in den Ressorts tätig sind, aktiv für Frauenbelange oder möchten dies. Und nicht alle Frauen, die für Frauenbelange arbeiten, haben von vornherein viel Frauenbewusstsein. „Schon deshalb nicht, weil sie oft zufällig als einzige Frau bestimmt werden oder um eine engagierte Frau zu verhindern", wie dies Rowhani (1991: 196) beschreibt. Aber trotz des Bekenntnisses zu Gender Mainstreaming, nach dem alle für Gleichstellungsbelange zuständig sind, sind es einzelne Frauen, die die Gleichstellungspolitik vorantreiben.

Mit institutionellen Regelungen der Berichtspflicht oder Bekenntnissen zur systematischen Berücksichtigung von Genderperspektiven in allen Bereichen und Ebenen der Politik sind die Weichen zur Unterstützung des Engagements von Einzelnen gestellt worden. Die Verschränkung von Gleichstellungspolitik und Personalpolitik ist in den Zielsetzungen des Frauenförderplans des Finanzministeriums und als Maßnahme des Sozialministeriums explizit enthalten. Aber in den meisten Frauenförderplänen ist dies kein Thema und die Verschränkung in der Praxis vermutlich noch weniger gegeben.

Wesentlich für die Wirksamkeit der Gleichstellung ist, dass sie auch kontrolliert wird. Dies erfolgt in Österreich durch die Berichtspflicht der Ministerien, setzt also auf die Öffentlichkeit als Kontrolle. Durch „naming and shaming“" soll Veränderung passieren. Aber nachdem das Monitoring primär auf die Partizipation von Frauen konzentriert ist und kein Vergleich der Ministerien unter Berücksichtigung des jeweiligen Kontextes durchgeführt wird, bleibt dies ein sehr ,weiches“ Kontrollinstrument. Zudem werden die Berichte von jenen gemacht, die zugleich zur Verantwortung gezogen werden, werden die Gleichbehandlungsberichte stärker als Beweis für die engagierte Arbeit verwendet, als dass diese genutzt werden, um Handlungsbedarf aufzuzeigen und Ressortverantwortliche zum Handeln zu bewegen. Dies betrifft sowohl die Umsetzungsanalysen als auch die enthaltenen Zielwerte der Frauenquoten. Wenn Ziele, nach denen die Ressorts bewertet werden, von ihnen 
selbst definiert sind, besteht ein Anreiz, die Ziele für eine hohe Chance der Zielerreichung möglichst niedrig zu halten oder aber die Ziele unkonkret $\mathrm{zu}$ formulieren. Mit den Gleichstellungsberichten erfolgt eine regelmäßige Berichterstattung an die PolitikerInnen, die aber ihre direkten Zugriffsmöglichkeiten für eine nachhaltige Gleichstellungspolitik im Bundesdienst nicht nutzen. Aber auch in den Ressorts resultieren aus den Berichten wenig praktische Konsequenzen; die Berichte werden teilweise nicht beachtet. Dementsprechend könnte das Potenzial der Gleichstellungspolitik im Bundesdienst durch Evaluation der Umsetzungspraxis oder auch durch ein verbessertes Monitoring, das auf die unterschiedliche Performance der Ressorts Bezug nimmt, verbessert werden.

Insgesamt muss aber hier nochmals betont werden, dass die Beurteilung der betrieblichen Gleichstellungspolitik hinsichtlich der Wirkungsmöglichkeiten des formalen Steuerungssystems erfolgte. Auch wenn staatliche Verwaltung wesentlich an gesetzlichen Regelungen und Verordnungen orientiert ist, ist davon auszugehen, dass in der Umsetzungspraxis formell verankerte Maßnahmen teilweise nicht relevant sind, die Erfolge der Gleichstellungspolitik auch informellen Normen und persönlichem Engagement zu verdanken sind. Doch welche Mechanismen dafür entscheidend sind, wie Gleichstellung in der Organisationskultur der Verwaltung integriert ist, wie Gleichstellungs- und Personalpolitik inhaltlich und personell verschränkt sind, wären spannende weiterführende Fragestellungen. 



\title{
$7 \quad$ Vorstellungen zu Gleichstellung und Geschlecht bei der Genese und Implementation betrieblicher Gleichstellungspolitik am Beispiel der Schweiz
}

\author{
Gesine Fuchs, unter Mitarbeit von Yvonne Debrunner
}

\section{1 $\quad$ Einleitung}

Dieses Kapitel nutzt die breitere Datenerhebung aus der schweizerischen Fallstudie sowie Daten zum gleichstellungspolitischen Agenda-Setting aus dem NFP60-Projekt, um beispielhaft Vorstellungen zu Gleichstellung und Geschlecht zu analysieren, wie sie sich in der Politik und in der Organisation „Bundesverwaltung“ zeigen. Solche Vorstellungen bestimmen Handlungsorientierungen und vorgeschlagene Lösungen mit: Welche Probleme werden als relevant, welche Ansätze als dringend und nützlich angesehen? Wann und wodurch ist betriebliche Gleichstellung erreicht? Innerhalb der ,vergeschlechtlichten Organisation“ (Acker 1990; Müller et al. 2013; vgl. Kapitel 7.2) prägen implizite Leitbilder und vorhandene organisationale ,vergeschlechtlichte Substrukturen" die Implementation der Politik und die Setzung von Schwerpunkten innerhalb der betrieblichen Politik mit. Zudem ist gemäß unserem in Kapitel 2 eingeführten „Schalenmodell“ das konkrete Steuerungsregime betrieblicher Gleichstellungspolitik in das Policy-Regime beruflicher Gleichstellungspolitik eingebettet. Beide sind wiederum durch das umfassendere nationale Geschlechterregime geprägt, das ökonomische, soziale, symbolische und kulturelle Normen, Werte und Praktiken des Geschlechterverhältnisses umfasst. Policy- und Gender-Regime nehmen also Einfluss auf die politisch vorgeschlagenen und durchsetzungsfähigen Programmatiken betrieblicher Gleichstellungspolitik. Um also Chancen und Restriktionen betrieblicher Gleichstellungspolitik fundierter einzuschätzen, werden hier gleichsam zwei Phasen betrieblicher Gleichstellung betrachtet: Erstens werden für Agenda-Setting und Politikformulierung die Entwicklung parlamentarischer Debatten und Vorstöße (= parlamentarischer Anträge) zur Gleichstellungspolitik in der Bundesverwaltung untersucht. Zweitens werden aus einigen Politikdokumenten und vor allem aus den ExpertInneninterviews in der Bundesverwaltung verschiedene Vorstellungen zur Gleichstellung der Geschlechter und von den Elementen einer entsprechenden betrieblichen Politik rekonstruiert. Welche Ideen zeigen sich und inwiefern schlagen sie sich sowohl im politischen Entstehungsprozess als auch in der Implementation nieder? Dabei geht es im engeren Sinne um Vorstellungen zur Gleichstellung der Geschlechter als Ziel der Organisation - wie Geschlecht sich in 
der Organisation konstituiert und wirkt, wurde nicht untersucht (vgl. Wilz 2013: 152).

Der folgende zweite Abschnitt behandelt die Entstehung und Weiterentwicklung des neuen Bundespersonalrechts von Ende der 1990er Jahre bis 2011: Wo werden in der politischen Debatte Geschlechterverhältnisse und betriebliche Gleichstellung angesprochen? Dafür werden parlamentarische Debatten und Vorstöße beschrieben und ausgewertet. Im dritten Abschnitt werden Vorstellungen zu Geschlecht und Gleichstellung vornehmlich aus den ExpertInneninterviews herausgearbeitet und diskutiert. Bei beiden Quellenarten - Debatte und Vorstöße einerseits und Interviews andererseits - wurde inhaltsanalytisch vorgegangen. ${ }^{1}$

\subsection{Geschlecht und Gleichstellung bei der Genese betrieblicher Gleichstellungspolitik}

\subsubsection{Betriebliche Gleichstellung und neues Bundespersonalrecht}

Eine erste Annäherung an im politischen Prozess wirksame Vorstellungen von Gleichstellung bietet der Blick auf die entsprechenden rechtlichen Grundlagen, hier das Bundespersonalrecht: Gesetze postulieren stets das normativ Gewünschte und „Normale“ und geben einen verbindlichen Interpretationsrahmen vor. Dabei sind sie selbst Produkt von Wahrnehmungen und Auseinandersetzungen (vgl. Baer 2004: 71f.). Das neue Bundespersonalgesetz (BPG) ersetzte 2002 das alte Beamtengesetz von 1927. An ihm ist zweierlei bemerkenswert: Erstens steht es ganz in der Tradition des New Public Management und zweitens enthält es nur wenige allgemeine Aussagen zur betrieblichen Gleichstellung.

Die Arbeiten zu einem neuen Bundespersonalrecht begannen 1993, und im Mai 1998 wurde die Vernehmlassung zum Entwurf des Bundesrates eröffnet, also die vorgeschriebene Konsultation bei Kantonen, Organisationen und weiteren Interessengruppen. Bereits im Dezember wurde die Botschaft des Bundesrates an die Eidgenössischen Räte geleitet. Das BPG formuliert allgemeine Prinzipien, während die Umsetzung durch Verordnungen und exekutive Weisungen geregelt ist. Es verschiebt Kompetenzen von der Legislative zur Exekutive - bzw. entflicht diese Kompetenzen. Es steuert vor allem mit Geboten und Zielformulierungen und macht nur wenige materielle Vorschriften. Die politischen Debatten um das neue Bundespersonalrecht waren heftig; von gewerkschaftlicher und linker Seite wurden vor allem die Abschaffung des Beamtenstatus, d.h. des umfassenden Kündigungsschutzes,

Vgl. Mayring (2010); die Interviews wurden mit maxqda kodiert und ausgewertet. 
und die Einführung von Leistungslöhnen scharf kritisiert. Zum Jahresanfang 2000 wurde das Gesetz verabschiedet. Dagegen wurde das Referendum ergriffen; in der entsprechenden Abstimmung im November 2000 wurde es allerdings mit einer Zweidrittelmehrheit gutgeheißen.

$\mathrm{Zu}$ den allgemeinen Zielformulierungen im Bundespersonalgesetz gehört das explizite Fördergebot für Gleichstellung und Chancengleichheit (Art. 4 Abs. 2d BPG). Das Gesetz anerkennt die Tatsache, dass Mitarbeitende auch Sorgearbeit leisten, mit der geschlechtsabstrakten Formulierung, die Arbeitgeber träfen Maßnahmen ,zur Schaffung von Arbeitsbedingungen, die dem Personal erlauben, seine Verantwortung in Familie und Gesellschaft wahrzunehmen" (Art. 4 Abs. 2i BPG). Damit sind beide Geschlechter angesprochen. Gleichstellung bedeutet aber auch besondere Förderung von Frauen. So nennt die Bundespersonalverordnung in der einzigen ,asymmetrischen“ Formulierung das Ziel, den Anteil von Frauen in Kaderpositionen zu erhöhen (Art. 5 Abs. 2f BPV). In der Botschaft des Bundesrates (also dem Text der Regierung, welcher den Konsultationsprozess einleitete) wurden die Gleichstellungsvorschriften des Entwurfs kaum begründet. Eventuell waren die Beratungen zum Gleichstellungsgesetz 1995 noch gegenwärtig. Grundsätzlich stützte das Parlament das Bestreben nach einem Rahmengesetz, das die meisten konkreten Regelungen dem Verordnungsweg überlässt, also Kompetenzen zur Exekutive verschob. Neben Änderungsanträgen zu Teilzeitarbeit, Betreuung kranker Kinder und Bereitstellung von Kinderbetreuungseinrichtungen sind zwei Anträge besonders erwähnenswert:

Die Sozialdemokratin Vreni Hubmann forderte, inspiriert von Vorschlägen der Schwulenorganisation Pink Cross, ein Bekenntnis ,für die Chancengleichheit der Lebensformen“" im Artikel zu den Grundsätzen der Personalpolitik (vgl. Amtliches Bulletin 1999, N 2055-2059). Damit sollten Diskriminierungen verhindert werden, die im ganzen Erwerbsleben von der Anstellung bis zur Beförderung immer wieder auftauchten. Zudem sollten die Lebenspartnerschaften von Mitarbeitenden anerkannt werden. Der Zusatz stelle die folgerichtige Umsetzung des Diskriminierungsverbots in Artikel 8 der neuen Bundesverfassung auf Gesetzesebene dar. Die bürgerlichen Gegenargumente tauchen fast identisch auch in späteren Debatten um eine Ausweitung des Diskriminierungsschutzes auf (vgl. Fuchs 2008b: 41f.): Die verfassungsrechtlichen Vorschriften reichten aus, und sexuelle Orientierung sei eine intime Privatangelegenheit, die den Arbeitgeber nichts angehe. Man sprach sich gegen eine Liste von schutzbedürftigen Personen aus; dies werfe nämlich die Frage auf, warum gerade jene und nicht eine andere benachteiligte Gruppe erwähnt wird. Einen solchen Zusatz könne man gar „mit Fug und Recht wieder als Diskriminierung anderer Gruppen betrachten." (Eugen David, CVP, Amtliches Bulletin 1999 N 2057) Ein solches Argument wurde auf die ebenfalls im Gesetz erwähnten Behinderten oder sprachlichen Minderheiten allerdings nicht angewendet. 
Ein weiterer Minderheitenantrag wollte in Artikel 14 zum Lohn die Bestimmung einfügen, der Bundesrat solle darüber wachen, dass weibliche Beschäftigte den gleichen Lohn erhalten und die Arbeitsbewertungsinstrumente nicht geschlechterdiskriminierend seien. ${ }^{2}$ Cécile Bühlmann von den Grünen begründete ihren Antrag mit der Erfahrung, dass bei der Lohneinstufung indirekte Diskriminierung vorkomme, weil übliche Bewertungsverfahren und die Entscheidungsspielräume von Vorgesetzten Kompetenzen von Frauen und wichtige Merkmale typischer Frauentätigkeiten unter- und solche von Männern überschätzten. Gegen solche Mechanismen sei auch die Bundesverwaltung nicht gefeit (Amtliches Bulletin 1999 N 2075). Dies gelte in besonderem Maße auch für Leistungslohnbestandteile, wurde in der Debatte mehrmals geäußert. Auch dieser Antrag wurde ,im Windschatten“ weiterer Minderheitsanträge abgelehnt, ohne dass es im Plenum Gegenargumente gab.

Die Debatte zeigt gewisse heteronormative Züge: Während sexuelle Orientierung als eine Privatangelegenheit, ja als ,intim“ definiert wird und des Diskriminierungsschutzes nicht bedarf, sind Bekenntnisse zur Chancengleichheit von Frauen und Männern hingegen grundsätzlich akzeptiert. Wenn diese allgemeinen und normativen Bekenntnisse aber konkretisiert werden sollten, war sich das Parlament uneinig und lehnte alle Änderungen ab. Diese Tendenz lässt sich auch in anderen nationalen Kontexten beobachten (vgl. Fuchs/Bothfeld 2011: 18).

\subsubsection{Konkretisierung der betrieblichen Gleichstellungspolitik: Parlamentarische Anträge 1996-2011}

Nach dem Inkrafttreten des Gesetzes wurden die dort enthaltenden Gleichstellungspostulate von Seiten des Bundesrates in Verordnungen und Weisungen konkretisiert. Kontinuierlich versuchen auch Parlamentsmitglieder, die Arbeitsbedingungen in der Bundesverwaltung über parlamentarische Vorstöße zu beeinflussen. Parlamentarische Vorstöße sind Anträge von einem oder mehreren Parlamentsmitgliedern bzw. Fraktionen, der Bundesrat möge ein Gesetz ausarbeiten oder den Handlungsbedarf in einem bestimmten Bereich prüfen und Bericht erstatten. Eine Parlamentsmehrheit muss einen Vorstoß überweisen, d.h. dem Bundesrat einen Auftrag erteilen. Dieser muss wiederum innerhalb einer bestimmten (zuweilen nicht eingehaltenen) Frist Bericht erstatten und das weitere Vorgehen vorschlagen. Vorstöße nehmen im schweizerischen Konkordanzsystem mit einer Allparteienregierung eine wichtigere Stellung ein als in den parlamentarischen Systemen der Nachbar-

2 Der Bundesrat, ,veille tout particulièrement à ce que les employés de sexe féminin perçoivent le même salaire et vérifie en permanence que les outils qu'il utilise pour l'évaluation du travail ne sont pas sexuellement discriminatoires.“ (Amtliches Bulletin 1999, N 2075) 
länder (Lüthi 2014: 175f.). ${ }^{3}$ Die gleichstellungsrelevanten Vorstöße beziehen sich bemerkenswerterweise zum größten Teil auf die betriebliche Gleichstellung. Diese Anträge und ihre Diskussion bieten einen guten Einblick in die Vorstellungen des Parlaments über eine angemessene Gleichstellungspolitik. Im Rahmen des Schweizer Forschungsprojekts wurde eine Analyse des gleichstellungspolitischen Agenda-Settings in den Eidgenössischen Räten zwischen 1996 und 2011 vorgenommen und dabei die Vorstöße zur Personalpolitik in der Bundesverwaltung genauer untersucht (Fuchs/Debrunner 2014).

Zwischen 1996 und 2011 nahm das gleichstellungspolitische Agenda-Setting in den Eidgenössischen Räten relativ und absolut zu: In jeder Legislatur wurden mehr Vorstöße eingereicht. Der Anteil der gleichstellungsrelevanten Vorstöße wuchs aber auch im Verhältnis zur Gesamtzahl dieser Anträge. Von den insgesamt 338 erfassten gleichstellungsrelevanten Geschäften waren etwa drei Viertel persönliche Vorstöße, die ebenfalls zu drei Viertel von Frauen eingebracht wurden (der Männeranteil stieg im Zeitverlauf immerhin von $16 \%$ auf $28 \%$ ). Ebenfalls ist typisch, dass etwa zwei Drittel der Initiativen aus dem linken Lager stammten (d.h. Sozialdemokratische Partei, Grüne und in geringem Maße die Partei der Arbeit). Dabei machten die Themen Erwerbsarbeit, Vereinbarkeit und Kinderbetreuung fast 50\% aller Vorstöße aus. Jedoch wurden auch „klassische“ Themen, die die Zweite Frauenbewegung seit ihren Anfängen beschäftigen, auf der Agenda, etwa häusliche Gewalt oder Abtreibung. ${ }^{4}$ Die Hälfte der Vorschläge bewegte sich im Bereich regulativer Politik, also rechtlicher Änderungen, was an sich eine große Spannbreite aufweist. Sie reicht von der Gewährung individueller Rechtsansprüche über Handlungspflichten bis zur Installierung von Berichtspflichten oder rechtlicher Vereinheitlichung. Ein knappes Fünftel setzte vor allem auf Überzeugung und jeweils etwa jeder zehnte Vorstoß wollte mit institutioneller Politik oder Vorbildwirkungen arbeiten.

Zwischen 1996 und 2011 gab es 18 parlamentarische Vorstöße und zwei Vorlagen des Bundesrates zur Gleichstellungspolitik in der Bundesverwaltung. Diese Geschäfte unterschieden sich von den restlichen gleichstellungsrelevanten Vorstößen vor allem im vorgeschlagenen Steuerungsprinzip, denn überwiegend wird hier auf die Vorbildwirkung gesetzt - Politikerinnen sehen

3 Mit einer Parlamentarischen Initiative kann der Entwurf zu einem Erlass oder dessen Grundzüge vorgeschlagen werden, wobei die Gesetzgebungsarbeiten in den zuständigen parlamentarischen Kommissionen erfolgen. Parlamentarische Initiativen werden in den Kommissionen vorberaten, bevor das Parlament über eine Auftragserteilung entscheidet. Motionen verlangen Erlass oder Änderung eines Gesetzes. Das unverbindlichere Postulat verlangt vom Bundesrat zu prüfen und zu berichten, ob ein Erlass oder eine Maßnahme getroffen werden soll. Mehr Informationen unter http://www.admin.ch.

42002 wurde die Fristenregelung beim Schwangerschaftsabbruch in einer Referendumsabstimmung angenommen. Seit 2004 sind typische Delikte im Bereich häuslicher Gewalt Offizial- und keine Antragsdelikte mehr. 


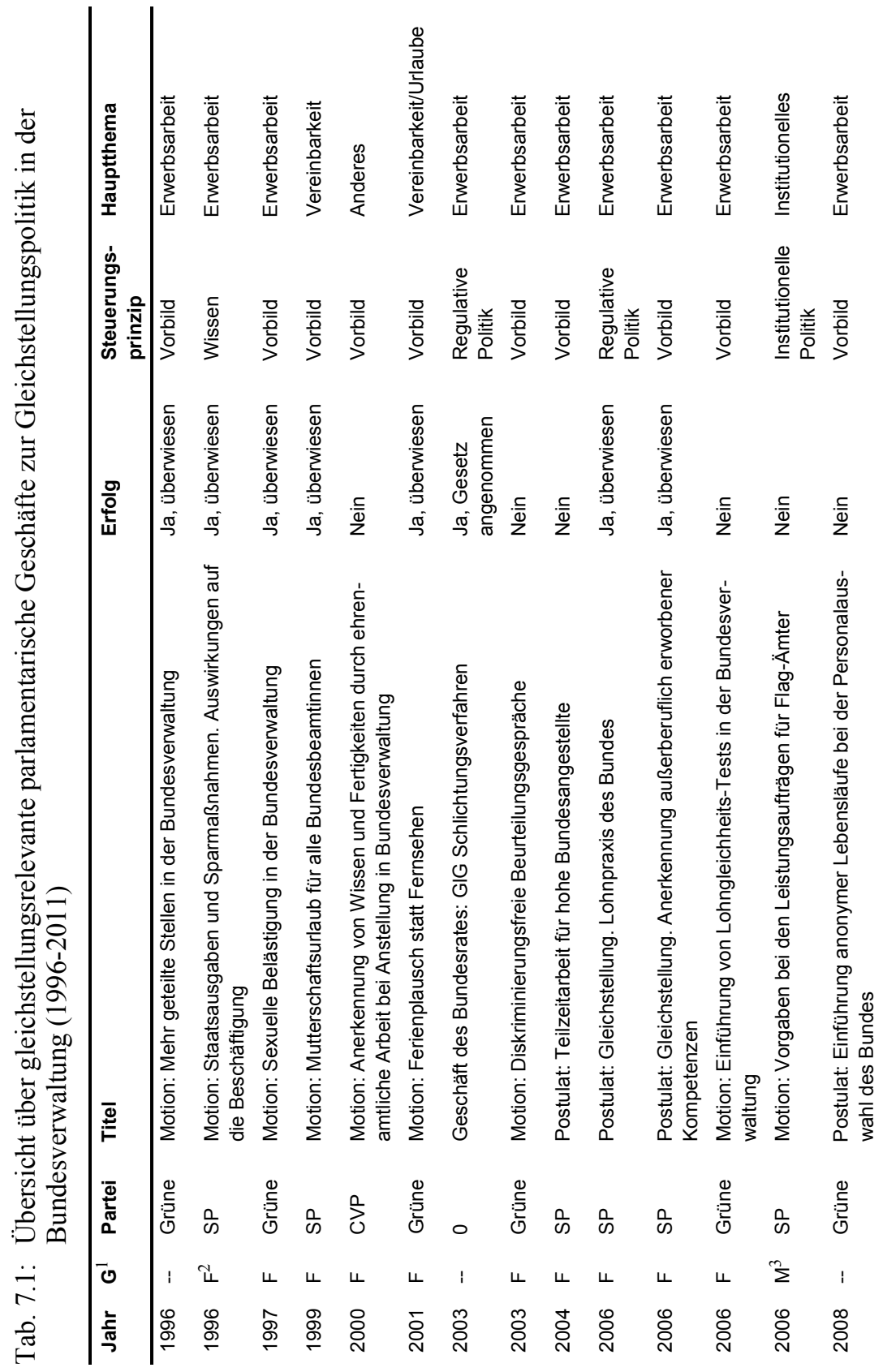




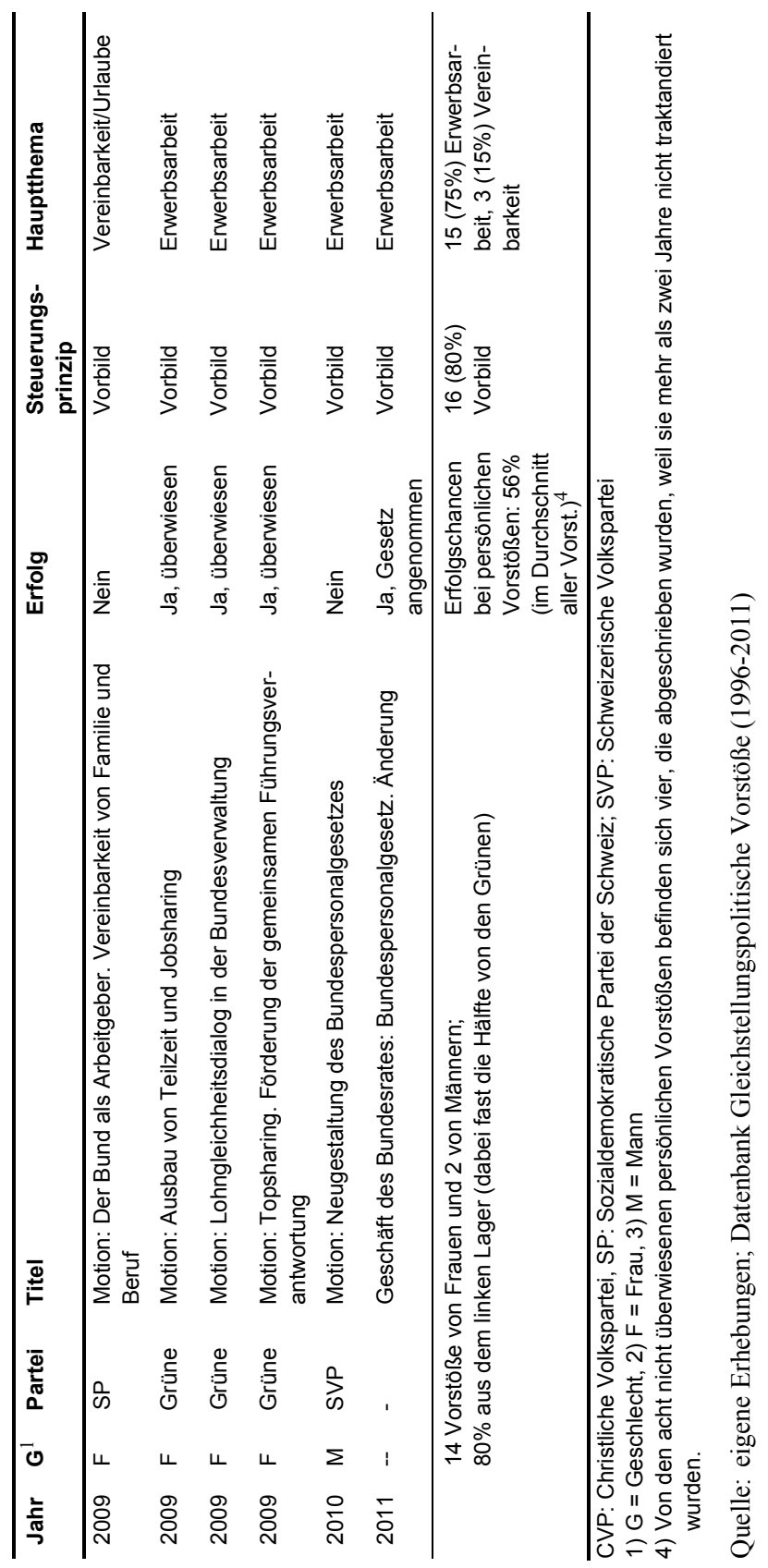


die Bundesverwaltung als Möglichkeit, gleichstellungspolitisch Sinnvolles zu erproben. Naturgemäß überwog thematisch die Erwerbsarbeit mit acht Anträgen. Hiervon beschäftigten sich vier Vorstöße mit Lohngleichheit, was die zentrale Stellung dieses Themas in der schweizerischen Gleichstellungspolitik unterstreicht.

\subsubsection{Frames für und gegen Gleichstellung in den Debatten zu betrieblichen Gleichstellungsmaßnahmen}

Welche Argumente für oder gegen Gleichstellung, welche Vorstellungen von Geschlecht lassen sich aus den eingereichten Texten und Begründungen, aus den Stellungnahmen des Bundesrates oder der Kommissionen sowie aus der parlamentarischen Diskussion herausarbeiten? Welche argumentativen Bezugsrahmen (Frames) verwenden die Politiker und Politikerinnen?

Die Methode der Frame-Analyse schöpft aus den Arbeiten des Soziologen Erving Goffman: Danach verwenden Menschen zur Organisation von Erfahrung und zur Orientierung in einer komplexen Welt Frames (= Rahmen) als grundlegende Orientierungsmuster. Frames sind ,schemata of interpretation that assist individuals in locating, identifying, receiving and labelling occurrences" (Goffman 1986: 21). Frames organisieren sozusagen die Wahrnehmung der Wirklichkeit. Welche Frames in einer Auseinandersetzung vorkommen und dominieren, wie sie transformiert und neue zusammengesetzt werden, hat Auswirkungen auf die Debatte und die als realistisch eingeschätzten Lösungsmöglichkeiten. Somit können politische AkteurInnen auch bewusstes Framing betreiben (Überblick bei Snow 2004). Kurz gesagt haben alle Menschen Frames, und Framing ist eine kollektive strategische Aufgabe einer sozialen oder politischen Bewegung, diese Frames zu verändern. Im Folgenden sind mit Frames diese Deutungsmuster gemeint. Einzelne Argumente und Aussagen in den analysierten Quellen wurden solchen Mustern zugeordnet. Die Aggregation der Argumente resultierte in fünf Pro-Frames und fünf Contra-Frames. ${ }^{5}$

Die Analyse der erfassten Vorlagen zeigte, dass der Diskurs über gleichstellungsrelevante Themen in eingespielten Bahnen verläuft. Der Rahmen der Pro-Argumente ist ebenso klar abgesteckt wie derjenige der Contra-Argumente. Dies zeigte sich insbesondere daran, dass so gut wie alle Argumente problemlos einem der hier aufgeführten Frames zugeordnet werden konnten. Argumente, die andere Aspekte ansprechen, wurden nicht gefunden. Auffällig ist zudem, dass wenig normative Argumente verwendet werden. In vielen der untersuchten Fälle läuft die Debatte auf Fragen der Finanzierung oder der Zuständigkeit hinaus. So antwortet der Bundesrat auf gleichstellungsrelevan-

5 Vgl. auch im Folgenden Fuchs/Debrunner (2014: 24-38). Die Frameanalyse hat Yvonne Debrunner durchgeführt. 
Tafel 7.1: Übersicht über Pro-Frames bei gleichstellungspolitischen Geschäften

\begin{tabular}{l}
\hline Pro-Frames und einzelne Argumente \\
\hline Gleichstellung bringt ökonomischen Nutzen \\
\hline Frauen stellen heute schon die Mehrheit der Studierenden, durch den demographischen Wandel \\
müssen Frauen in den Arbeitsmarkt integriert werden, dies auch anstatt zusätzlicher politisch \\
brisanter Einwanderung. Mehr Frauen in Erwerbsarbeit bringen volkwirtschaftlichen Nutzen auch \\
in den Sozialwerken, Teilzeitarbeit erhält Qualifikationen und erhöht die Produktivität.
\end{tabular}

Die Vereinbarkeit von Familie und Beruf, die Work-Life-Balance, muss gefördert werden

Eltern sollen sich nicht für Beruf oder Familie entscheiden müssen und gemeinsam Verantwortung für den Familienunterhalt übernehmen. Es entspricht neuen Lebensentwürfen, gibt weniger Stress und bringt gleichberechtigte Väter hervor.

\section{Der Bund soll Vorbild in seiner Gleichstellungspolitik sein}

Der Bund hat eine Vorbildfunktion und soll damit Unternehmen zur Nachahmung ermuntern. Er muss hinsichtlich seiner politischen Forderungen (z.B. Lohngleichheit) glaubhaft wirken.

\section{Der Bund soll ein attraktiver Arbeitgeber sein}

Der Bund muss wegen des Arbeitskräftemangels aufgrund des demografischen Wandels Anstrengungen unternehmen, um ein attraktiver und familienfreundlicher Arbeitgeber zu bleiben. Eine funktionierende Verwaltung mit qualifiziertem Personal ist im Interesse der Bevölkerung.

\section{Gleichstellung ist noch nicht erreicht}

Gleichstellung muss auch tatsächlich umgesetzt werden, etwa im Bereich Lohngleichheit oder bei der Leistungsbeurteilung. Außerberufliche Kompetenzen werden bisher kaum berücksichtigt, Frauen sind durch Hausarbeit stärker belastet; Maßnahmen gegen sexuelle Belästigung fehlen.

Quelle: eigene Zusammenstellung

te Vorstöße häufig mit dem Argument, dass er dem Anliegen zwar grundsätzlich zustimme, dass das Thema aber nicht (nur) in seine Zuständigkeit falle. Insbesondere die Gegner gleichstellungsrelevanter Vorstöße wagen sich nicht auf das dünne Eis normativer Debatten. Es wurde kein Fall gefunden, in welchem Gleichstellung normativ in Frage gestellt wurde. Die am häufigsten angewendeten Frames der Gegner sind stattdessen, dass Gleichstellung bereits erreicht sei und der Vorstoß daher unnötig, oder dass das Anliegen zu teuer sei. Des Weiteren werden die Forderungen als illusorisch oder als schlecht für die Privatwirtschaft zurückgewiesen. Auch die Befürworter legen, vermutlich um Mehrheiten zu gewinnen, einen Fokus auf ökonomische Argumente. Das Framing von gleichstellungsrelevanten Vorstößen als volkswirtschaftlich sinnvoll ist weit verbreitet. Des Weiteren wird argumentiert, der Bund solle ein Vorbild bzw. ein attraktiver Arbeitgeber sein. Noch am ehesten in eine normative Richtung gehen die Frames, welche die Vereinbarkeit von Familie und Beruf betonen oder herausstreichen, dass Gleich- 
Tafel 7.2: Übersicht über Contra-Frames bei gleichstellungspolitischen Geschäften

\begin{tabular}{l}
\hline Contra-Frames und einzelne Argumente \\
\hline Gleichstellung ist bereits erreicht \\
\hline Die Forderungen sind überholt und längst erreicht; es gibt bereits viele Maßnahmen. Heute \\
werden Frauen nicht mehr diskriminiert, sondern allenfalls Männer. Lohngleichheit ist bereits \\
rechtlich festgeschrieben, und das Lohnsystem des Bundes lässt keine Diskriminierung zu. \\
Transparenz im Lohnsystem oder Berücksichtigung von außerberuflichen Kompetenzen gibt es \\
bereits.
\end{tabular}

\section{Die Maßnahmen sind zu teuer}

Hoher finanzieller Mehraufwand für den Steuerzahler, kein Nutzen, nur zusätzlicher Aufwand. Jobsharing ist ökonomisch ineffektiv. Ein Vaterschaftsurlaub wirkt preistreibend und ist daher nur auf freiwilliger Basis einzuführen. Parlamentarische Budgetbeschränkungen erlauben keine Umsetzung gewünschter Maßnahmen.

\section{Die Maßnahmen sind illusorisch}

Der Staat kann nicht für alle persönlichen Bedürfnisse verantwortlich sein. Forderungen sind illusorisch, denn beispielsweise kann Führungsverantwortung nicht geteilt werden.

\section{Die Maßnahmen fallen nicht (nur) in die Zuständigkeit des Bundes}

Die Umsetzung liegt auch in der Zuständigkeit der Departemente und Ämter, diese müssen selbst entscheiden, ob sie Teilzeitstellen anbieten. Ebenso sollte innen die Entscheidung über Lohngleichheitstests überlassen bleiben. Der Bundesrat will nicht in die Arbeitsorganisationen öffentlicher Unternehmungen eingreifen.

\section{Schlecht für die Privatwirtschaft}

Ein Vaterschaftsurlaub könnte Begehrlichkeiten bei Kantonsangestellten und in der Privatwirtschaft wecken. Wenn sich Klein- und Mittelunternehmen inn nicht leisten können, führt das zu ungleichen Rekrutierungsbedingungen.

Quelle: eigene Zusammenstellung

stellung nach wie vor nicht erreicht sei. Diese Frames enthalten gewissermaßen eine implizite Normativität, welche sich im Wunsch nach Vereinbarkeit und Gleichstellung äußert.

$\mathrm{Ob}$ sich der Gebrauch der erwähnten Frames über die Zeit verändert, ist wegen der geringen Zahl der Vorstöße schwierig zu beurteilen. Am ehesten lassen sich Verschiebungen im Gebrauch von Argumentationen mit dem Aufkommen neuer Themen in Verbindung bringen - so erscheint das Argument, ein Vorstoß sei schlecht für die Privatwirtschaft, erst mit der Forderung eines Vaterschaftsurlaubs. Innerhalb des sehr wichtigen Frames zum wirtschaftlichen Nutzen hat eine Verschiebung bei den einzelnen Argumenten stattgefunden. Während zu Beginn des untersuchten Zeitraums der Fokus auf dem generellen volkswirtschaftlichen Nutzen von Teilzeitarbeit lag (z.B. die Motion der Grünen Fraktion 1996), scheint unterdessen die berufliche In- 
tegration hoch qualifizierter Frauen zentral zu sein, etwa bei der Förderung des sogenannten Top-Sharings, also geteilter Führungsverantwortung (Motion von Brigit Wyss, Grüne 2009). Argumentationen, die mehr Frauen in Führungspositionen fordern, betonen auch, dass Frauen schon die Mehrheit an den Studierenden stellen oder Teilzeitarbeit die Qualifikationen erhält. Diese Beobachtung reiht sich ein in den allgemeinen Trend der Ökonomisierung der Gleichstellungsfrage und der Fokussierung auf gut gebildete Frauen (vgl. dazu Leitungsgruppe 2014: 32f.; Widmer et al. 2014).

Beim Vereinbarkeits-Frame ist ein deutlicher Wandel feststellbar. Vereinbarkeit von Familie und Beruf ist durchgängig seit Mitte der 1990er Jahre ein wichtiges Thema. Obwohl es sich per se nicht auf ein bestimmtes Geschlecht bezieht, wurde es früher jedoch oftmals implizit im Zusammenhang mit Frauen verwendet. Dabei wurde häufig betont, dass dasselbe Argument für Männer ebenfalls gelte, diese wurden aber gewissermaßen nachträglich in die Argumentation eingeschlossen. ${ }^{6}$ Ein neues Element des VereinbarkeitsFrames stellt daher die explizite Betonung von Vereinbarkeit für Männer dar. Diese äußert sich insbesondere in Zusammenhang mit dem Vaterschaftsurlaub: Väter gewinnen eine eigenständige Bedeutung in der Debatte.

Bezüglich der Contra-Argumente fällt zudem auf, dass sich aktiver Widerstand aus den Reihen der Parlamentarier gegen Gleichstellungsmaßnahmen in der Bundesverwaltung erst seit 2006 regt. Zuvor stimmten die Gegner in den Abstimmungen zwar mit „Nein“, meldeten sich aber in der parlamentarischen Debatte nicht zu Wort. So wird erst ab 2006 offen in der Debatte davon gesprochen, dass die Forderungen überholt seien, die Anliegen bereits umgesetzt oder mittlerweile Männer diskriminiert würden. Ebenfalls Kennzeichen des aufkommenden Widerstands ab 2006 ist der neue Kosten-Frame.

Vermutlich sind die aufkommenden Gegenargumentationen ein Zeichen dafür, dass gerade auch Vereinbarkeitsfragen größere Sympathien im Parlament genießen und die Gegner eine Überweisung an den Bundesrat nun aktiv verhindern müssen. Tatsächlich sind von allen gleichstellungsrelevanten Vorstößen im Zeitraum 2006/11 ganze 49 zu Vereinbarkeit und Urlauben (verglichen mit acht Vorstößen 1996/2000 und 14 2001/05). Auch die FDP (Freisinnig-demokratische Partei) und die CVP (Christliche Volkspartei) nehmen sich nun dieses Themas an: Von den gleichstellungspolitischen Vorstößen der CVP sind 16 oder $42 \%$ zur Vereinbarkeit, bei der FDP sind es zehn oder 59\% ihrer Vorstöße, während es im linken Lager ein deutlich geringerer Anteil ist.

6 Exemplarisch zeigt dies das folgende Zitat auf: „Doch die meisten berufstätigen Frauen und auch zunehmend Männer, die Kinder betreuen oder Eltern pflegen, möchten diesen wichtigen Aufgaben mithilfe einer Teilzeitstelle oder dank Jobsharing besser gerecht werden.“ (Christine Egerszegi-Obrist, Parl. Debatte SR zu Geschäft 09.3066, 25.11.2009, verfügbar unter www.parlament.ch/d/suche/seiten/curia-vista.aspx) 
2006 und 2009 wurden jeweils Lohngleichheitstests in der Bundesverwaltung gefordert. Während 2006 der grüne Vorstoß diskussionslos vom Nationalrat abgelehnt wurde, erhielt die praktisch identische Version 2009 eine Mehrheit in beiden Räten. Dabei wurde nur 2009 im Plenum gegen den Vorstoß argumentiert: Die Forderung wurde im Wesentlichen als überholt und zu teuer zurückgewiesen. Die Überweisung erfolgte mutmaßlich wegen der positiven Empfehlung des Bundesrates. Dieser hatte im Nachgang der Evaluation des Gleichstellungsgesetzes (AG 2005 und Vorlage 07.031 des Bundesrates) die freiwillige Maßnahme des Lohngleichheitsdialogs propagiert und stand so unter Druck, dies auch ,in den eigenen Reihen“ durchzuführen. Ebenfalls von Relevanz könnte die breitere Argumentation der BefürworterInnen gewesen sein.

Generell müssen jedoch die BefürworterInnen von Vorstößen mehr als GegnerInnen argumentieren. Tabelle 7.2 gibt einen Überblick, welche Argumente wie häufig verwendet wurden und wie häufig die so debattierten Vorstöße überwiesen oder abgelehnt wurden.

Tab. 7.2: Frames: Anzahl Verwendungen und Erfolgsrate in debattierten Vorstößen zur Bundesverwaltung

\begin{tabular}{|c|c|c|c|c|}
\hline Pro-Frames & $\begin{array}{l}\text { \# Verwendun- } \\
\text { gen dieses } \\
\text { Frames }\end{array}$ & $\begin{array}{l}\text { \# überwiesene } \\
\text { Vorstöße mit } \\
\text { diesem Frame }\end{array}$ & $\begin{array}{l}\text { \# nicht über- } \\
\text { wiesene } \\
\text { Vorstöße mit } \\
\text { diesem Frame }\end{array}$ & $\begin{array}{l}\text { \% überwiese- } \\
\text { ner Vorstöße } \\
\text { mit diesem } \\
\text { Frame }\end{array}$ \\
\hline $\begin{array}{l}\text { Gleichstellung noch } \\
\text { nicht erreicht }\end{array}$ & 11 & 6 & 5 & 54,5 \\
\hline Vorbild & 9 & 5 & 3 & 55,6 \\
\hline Vereinbarkeit & 6 & 4 & 2 & 66,7 \\
\hline Volkswirtsch. Nutzen & 5 & 3 & 2 & 60,0 \\
\hline Attrakt. Arbeitgeber & 4 & 2 & 2 & 50,0 \\
\hline Contra-Frames & $\begin{array}{l}\text { \# Verwendun- } \\
\text { gen dieses } \\
\text { Frames }\end{array}$ & $\begin{array}{l}\text { \# überwiesene } \\
\text { Vorstöße mit } \\
\text { diesem Frame }\end{array}$ & $\begin{array}{l}\text { \# nicht über- } \\
\text { wiesene } \\
\text { Vorstöße mit } \\
\text { diesem Frame }\end{array}$ & $\begin{array}{l}\text { \% nicht über- } \\
\text { wiesener } \\
\text { Vorstöße mit } \\
\text { diesem Frame }\end{array}$ \\
\hline $\begin{array}{l}\text { Gleichstellung } \\
\text { bereits erreicht }\end{array}$ & 8 & 3 & 5 & 62,5 \\
\hline Zu teuer & 6 & 3 & 3 & 50,0 \\
\hline $\begin{array}{l}\text { Nicht nur Zuständig- } \\
\text { keit des Bundes }\end{array}$ & 5 & 1 & 4 & 80,0 \\
\hline $\begin{array}{l}\text { Illusorische Forde- } \\
\text { rungen }\end{array}$ & 1 & 1 & 0 & 0,0 \\
\hline
\end{tabular}

Quelle: eigene Zusammenstellung 
Die Tabelle veranschaulicht, dass die BefürworterInnen am häufigsten den Gleichstellung-noch-nicht-erreicht-Frame verwenden, der bei elf der 18 Parlamentsgeschäfte erwähnt wird. Auffällig ist, dass er in sechs dieser elf Fälle allein, also als einziger Pro-Frame, vorkommt. Dass Gleichstellung noch nicht erreicht ist, scheint also für die Initiantinnen in vielen Fällen eine ausreichende Begründung für die Forderung von Maßnahmen zur ihrer Erreichung zu sein. Es zeigt sich aber, dass dies nicht für das gesamte Parlament zutrifft. Indizien dafür liefert die Tatsache, dass der Gleichstellung-nochnicht-erreicht-Frame in Kombination mit anderen Frames viel mehr Erfolg bringt als allein. So wurden von den sechs Vorstößen, bei denen er alleine vorkommt, nur zwei überwiesen, d.h. der Bundesrat beauftragt. Von den fünf, in welchen er in Kombination mit anderen Frames erscheint, wurden vier überwiesen. Die zwei beliebtesten Frames der GleichstellungsgegnerInnen sind der Gleichstellung-bereits-erreicht-Frame und der Zu-teuer-Frame. Die letzte Spalte stellt die Erfolgsrate der unterschiedlichen Frames dar. Dabei ist der Volkswirtschaftlicher-Nutzen-Frame am stärksten mit Erfolg verknüpft. Das mag daran liegen, dass mit diesem Argument breite Kreise im Parlament angesprochen werden können; auch solche, welche nicht primär an Gleichstellung als normativem Ziel interessiert sind. Von den Contra-Frames ist der Gleichstellung-bereits-erreicht-Frame mit einer Rate von $62,5 \%$ am erfolgversprechendsten. ${ }^{7}$

\subsubsection{Zusammenfassung: Eingespielte Debatten ohne offene normative Konflikte}

Die hier analysierten Debatten zur Gleichstellung in der Bundesverwaltung verlaufen in eingespielten Bahnen mit klaren Pro- und Contra-Argumenten. Normative Argumente werden so gut wie keine verwendet - erst recht nicht von der Contra-Seite. Verglichen mit dem gesamten Korpus der gleichstellungspolitischen Vorstöße haben die Bundesverwaltungsvorstöße überdurchschnittliche Chancen, überwiesen und weiter bearbeitet zu werden. Dabei aktiviert die Pro-Seite durchschnittlich doppelt so viele Frames wie die Contra-Seite; tendenziell sind die Chancen auf Überweisung höher, wenn mehr Argumente in Begründung und Debatte verwendet werden. Erst ab 2006 wird aktiver Widerstand sichtbar: Vorher wurden Vorstöße häufig einfach diskussionslos nicht überwiesen (oder nicht traktandiert). Ab 2006 wird besonders prominent gegen eine Überweisung eingewendet, die Gleichstellung sei schon erreicht oder die Kosten seien zu hoch. Diese Begründungsbedürftigkeit kann ein Hinweis darauf sein, dass mehr Parlamentsmitglieder solchen

7 Zwar hat der Nicht-nur-Zuständigkeit-des-Bundes-Frame mit $80 \%$ die höchste Erfolgsrate, doch kommt er hauptsächlich dadurch zustande, dass der Bundesrat seinen ablehnenden Antrag damit begründet und das Parlament dem Antrag häufig folgt. 
gleichstellungspolitischen Vorlagen zugeneigt sind und auch im bürgerlichen Lager eine Ablehnung kein „Selbstläufer" mehr ist. Im Parlament ist offenbar umstritten, inwieweit Gleichstellung erreicht ist bzw. ob dieses Ziel bereits bei formaler Gleichbehandlung als umgesetzt angesehen werden kann oder ob die faktische Gleichstellung als Maßstab dienen soll.

Gleichstellung in der Bundesverwaltung ist vor allen Dingen als Frage der Arbeitsbedingungen und ihrer Verbesserung definiert. Damit ist betriebliche Gleichstellungspolitik ein klar linkes und gewerkschaftliches Anliegen, das von niemandem aus dem bürgerlichen Lager aktiv aufgegriffen wird. Die Vorstöße weisen dem Bundesrat die Umsetzungsverantwortung für die Gleichstellung zu. Langsam werden auch Männer explizit in die Argumente einbezogen, und zwar beim Vereinbarkeitsframe. Intersektionalität wird weder implizit noch explizit aufgeworfen; Fragen sexueller Orientierung oder sozialer Herkunft spielen keine Rolle. Die Bearbeitung von Vereinbarkeitsfragen hat zudem einen heteronormativen Fokus der Gleichstellungsfrage in der Bundesverwaltung zur Folge. Zudem lässt sich zeigen, dass die ökonomischen Begründungen für mehr Gleichstellungsmaßnahmen im Zeitverlauf tendenziell an Bedeutung gewinnen, sie mit parlamentarischem Erfolg verknüpft sind und dass dadurch der Fokus auch noch stärker auf Eliten - wie Frauen in Führungspositionen - gelegt wird. Auch innerhalb der Bundesverwaltung sind die ökonomischen Bezugsrahmen stark präsent, wie im nächsten Abschnitt zu sehen ist.

\subsection{Ziele der Gleichstellung in Programmen und Vorstellungen der Beschäftigten}

Bei der Schweizer Länderstudie wurden insgesamt vier Ämter näher untersucht. Eine Vorannahme bei der Auswahl der Ämter war, dass der Einschluss gleichstellungspolitischer Arbeiten oder Projekte in ein politisches Tätigkeitsfeld sich stärkend auf die zur Verfügung gestellten Ressourcen und die Verbreitung konkreter Maßnahmen auswirkt: Gleichstellungsrelevante Tätigkeiten oder Projekte in einem Amt liefern Akteuren und Einzelpersonen diskursive und legitimatorische Anknüpfungspunkte an eine interne Chancengleichheitspolitik sowie Anlässe zur Reflexion. Somit wäre bei ,gleichstellungsrelevanten“ Politikinhalten eine stärkere Chancengleichheitspolitik zu erwarten. Wo solche Tätigkeiten oder Projekte fehlen, wäre das Engagement entsprechend schwächer oder hätte höhere Hürden zu überwinden. Die Direktion für Entwicklung und Zusammenarbeit (DEZA) und das Staatssekretariat für Wirtschaft (SECO) wurden als Ämter mit viel gleichstellungsrelevanter inhaltlicher Arbeit identifiziert: In der DEZA ist Geschlechtergerechtigkeit seit langem ein Ziel der entwicklungspolitischen Arbeit; im SECO werden 
zahlreiche relevante Fragen behandelt. So war das Amt am Zustandekommen des Lohngleichheitsdialogs beteiligt oder an Präventionsprogrammen zu sexueller Belästigung ebenso wie an der Erarbeitung von Handreichungen zur Vereinbarkeit in Klein- und Mittelunternehmen (KMU).

Pointiert kann davon gesprochen werden, dass Gleichstellung in den Ämtern vor allem als Funktion guter Personalpolitik gefasst wird, was Vereinbarkeit von Familie und Beruf sowie mehr Frauen in Führungspositionen umfasst. Eine Ausnahme ist die untersuchte DEZA, die deshalb in Abschnitt 7.5 näher analysiert wird. Dort ist ein breiteres Verständnis vorhanden, bei dem auch die Organisation und ihre Kultur als wichtiges Feld von Gleichstellung genannt und reflektiert werden. Anders als in der politischen Debatte wird die Bundesverwaltung kaum als Vorbild für gute Gleichstellungspolitik gesehen, sondern mit den Maßnahmen geht es eher darum, als Arbeitgeber attraktiv zu bleiben und ,mithalten zu können“. Dabei sind auch fast keine negativen Einschätzungen der umgesetzten Maßnahmen zu verzeichnen.

Sehr pointiert tritt die Fokussierung von Gleichstellung als Vereinbarkeitsfrage in den ExpertInneninterviews hervor: Die Hälfte der Interviewten, unabhängig von ihrer Funktion, nennt Vereinbarkeitsmaßnahmen und besonders förderliche Arbeitszeitarrangements wie Teilzeitarbeit oder Telearbeit als wichtig für die Gleichstellung der Geschlechter. Gerade der technologische Fortschritt, der die Verbreitung der Telearbeit gefördert hat, wird auch für den Kulturwandel und die bessere Akzeptanz von Teilzeit- und Telearbeit verantwortlich gemacht. Einige erwähnen explizit, dass beide Geschlechter in der Lage sein sollten, Sorgearbeit zu übernehmen, vor allem die Betreuung kleiner Kinder. In einigen Überlegungen werden Vereinbarkeitsregelungen darauf zugespitzt, dass sie eine gute Maßnahme sind, um qualifiziertes Personal zu rekrutieren und zu halten. Damit ist Gleichstellungspolitik tendenziell kein Wert an sich, sondern eine Funktion guten Personalmanagements. Diese Argumentation findet sich auch in den Weisungen des Bundesrates zur Chancengleichheit wieder:

„1.2 Ziel dieser Weisungen ist es, die Chancengleichheit und die tatsächliche Gleichstellung von Frau und Mann am Arbeitsplatz zu verwirklichen. Das weibliche und männliche Human- und Wissenspotenzial soll in allen Bereichen optimal gefördert, eingesetzt und genutzt werden. Dadurch sollen die Konkurrenzfähigkeit und die Attraktivität des Bundes als Arbeitgeber gestärkt werden“ (vgl. http://www.admin.ch/ch/d/ff/2003/1435.pdf; Zugriff: 12.01.2013).

Ein weiterer wichtiger Bezugspunkt für erfolgreiche Gleichstellungspolitik ist der Anteil von Frauen in Führungspositionen. Ganz allgemein sprechen die ExpertInnen von Gleichstellung als einem Thema, das vor allem qualifizierte Beschäftigte und „High Potentials“ mit Universitätsausbildung betrifft. Beschäftigte in niedrigeren Lohnklassen wurden kaum je erwähnt: Die Weiterqualifizierung in Sekretariatsberufen wurde Mitte der 1990er Jahre noch in 
Programmen erwähnt, etwa im Frauenförderprogramm 1996-1999 des Personalsamts oder im Entwicklungsplan der DEZA 1997. Heute taucht dieses Ziel in Dokumenten und in den Interviews nicht mehr auf. Dieser Klassen-Bias ist unbewusst. Nur GewerkschaftsexpertInnen und eine ehemalige Gleichstellungsbeauftragte, die heute für eine Gewerkschaft arbeitet, erwähnen Beschäftigte ohne Universitätsabschluss als Zielgruppe für Gleichstellungsmaßnahmen. Sie sei schon damals allerdings mit ihren Vorgesetzten uneins gewesen: Gleichstellung solle keine elitäre Angelegenheit sein, sondern Maßnahmen für alle zugänglich. In diesem Zusammenhang seien noch das Reinigungspersonal im Bundesdienst erwähnt - eine typische Frauentätigkeit: Es ist im Unterschied etwa zum Deutschen Bundestag nicht an private Firmen ausgelagert. Es ist in der untersten Lohnklasse eingereiht, hat aber schlechtere Lohnsteigerungsmöglichkeiten als andere Lohnklassen und nur einen eingeschränkten Zugang zu Leistungsprämien. ${ }^{8}$ Putzen ist also eine Tätigkeit zweiter Klasse, auch wenn die Verordnung Ausdruck eines Kompromisses zwischen Arbeitgebern und Gewerkschaften ist, der als Alternative zur Auslagerung der Reinigung an private Firmen geschlossen worden sein mag.

Neben Vereinbarkeitsregelungen und Frauenförderung in Führungspositionen wurde in den vier untersuchten Ämtern nur in der DEZA auf die Wichtigkeit von Organisationsstrukturen und -routinen für die Gleichstellung hingewiesen. Die dortigen Gleichstellungsbeauftragten definierten betriebliche Gleichstellungspolitik als Organisationsentwicklung; die Programme von DEZA und später vom Gesamt-Außenministerium sind aus dieser Perspektive geschrieben.

\subsection{Gleichstellung durch Angleichung?}

Wodurch kann Gleichstellung am Arbeitsplatz erreicht werden? Unisono geteilt wird die Auffassung, dass Nichtdiskriminierung die Grundvoraussetzung ist, auf die sich alle Beteiligten auch verständigen können. Sodann gibt es unterschiedliche Meinungen. Weit verbreitet ist die Überlegung, dass Geschlechtergleichheit oder Chancengleichheit Gleichbehandlung bedeute:

„Also wie gesagt, es ist eben wirklich Chancengleichheit und nicht die Bevorzugung eines
Geschlechts. Wir haben einen Frauenanteil von rund $45 \%$; wir haben im unteren, im mittle-
ren und im oberen Kader Frauen; selbst die Staatssekretärin, welche das SECO leitet, ist
eine Frau. Für uns spielt es also einfach wirklich keine Rolle, ob jemand Mann oder Frau
ist. Das war noch nie ein Thema. Wir haben auch die Lohnvergleiche mitgemacht: Für
gleiche Arbeit gibt es - bei gleichen Voraussetzungen - auch gleiche Löhne, unabhängig
vom Geschlecht.“ (Interview)

Verordnung über das Unterhaltsreinigungspersonal (SGS 172.220.111.7). 
In dieser Sichtweise wird sich Gleichstellung daher auch gleichsam „organisch" entwickeln, wenn sich Personalabteilungen auf Gleichbehandlung und Nichtdiskriminierung verpflichten und wenn sie gesunden Menschenverstand und die anerkannten Prinzipien des Personalmanagements nutzen. Geschlechterungleichheiten werden nicht als ,riesiges Problem“ wahrgenommen: Der Personalbestand werde allmählich immer ausgewogener werden, auch weil Frauen heute wesentlich besser ausgebildet seien als vor zehn Jahren und weil der Arbeitsmarkt ohne weibliche Beschäftigte gar nicht mehr funktionieren könne.

Nur zwei Personen erwähnten klassische Differenz-Argumente, vertraten also die Auffassung, dass Frauen und Männer jeweils anders denken und andere persönliche Eigenschaften haben. Einige ExpertInnen äußerten darüber hinaus, dass Frauen benachteiligt sein könnten, weil sie weniger selbstbewusst seien und weil sie weniger als Männer bereit seien, den Preis für eine Karriere zu bezahlen. Damit kommt implizit die Vorstellung zum Ausdruck, dass Frauen nicht diskriminiert werden, wenn sie bereit sind, Karriere zu machen. Karrierewege oder -anforderungen werden im Zusammenhang mit Gleichstellung nicht in Frage gestellt. Diese Vorstellung könnte als „Gleichstellung durch Angleichung" bezeichnet werden.

Bessere Ausbildung von weiblichen Fachkräften, Nichtdiskriminierung und Gleichbehandlung reichen jedoch nicht aus; durchgängig in allen Ämtern wurden weitere wichtige Faktoren für eine erfolgreiche Gleichstellungspolitik genannt. Vor allem kultureller Wandel und die Work-Life-Balance der Beschäftigten sind relevant: Dazu brauche es zur Umsetzung der Maßnahmen im Bereich der Arbeitszeit Vertrauen. Vereinbarkeit und mehr Frauen im Kader seien heute gesellschaftlich gut akzeptiert, was es einfacher mache, eine starke „Gleichstellungskultur“ auch zu leben. Im Alltagsbewusstsein der Befragten ist zudem die Sicht verankert, dass ein Bekenntnis der Führungsebene - einer Amtsleitung oder der Bundesrätin -, dem auch Taten folgen, etwa bei der Besetzung von Stellen, unbedingt notwendig sind. Mehrere Befragte erwähnten eine gute Durchmischung bzw. eine gewisse Repräsentativität des Personals hinsichtlich Geschlecht, Sprachgruppe oder Behinderung als sehr nützlich für die Arbeit im Team. ${ }^{9}$

\subsection{Direktion für Entwicklung und Zusammenarbeit (DEZA) als Ausnahme}

Die DEZA wird im Folgenden separat analysiert, da sich dort ein Gleichstellungsverständnis zeigt, welches sich deutlich von den anderen schweizeri-

9 Bezeichnenderweise wurde dabei die Migrationsbevölkerung nie erwähnt. 
schen Ämtern unterscheidet. Hier wird die These vertreten, dass sich dies auf eine lange Tradition von Gleichstellungspolitik und Gender Mainstreaming zurückführen lässt.

\subsubsection{Lange Geschichte des Gender Mainstreaming}

In der Direktion für Entwicklung und Zusammenarbeit wird seit fast 20 Jahren das Gender Mainstreaming in den Programmen und Kooperationen mit ausländischen PartnerInnen propagiert und umgesetzt. Sie folgt damit den Diskussionen über die besten Entwicklungsansätze, die sich im ,gender and development"-Ansatz durchsetzten. Das bedeutet:

„Nur wenn die unterschiedlichen Rollen, Aufgaben und Bedürfnisse von Frauen und Männern berücksichtigt werden, wenn diese in der Projektlogik reflektiert sind und sich die geschlechterspezifischen Unterschiede auch in den Wirkungsberichten manifestieren, kann der Auftrag der Armutsbekämpfung wirkungsvoll umgesetzt werden." (Sancar 2011: 2)

Die DEZA hat dazu 2003 eine Gender Equality Policy verabschiedet und zusätzlich verschiedene Instrumente erarbeitet, wie Anleitungen, Beispiele guter Erfahrungen, Trainings sowie institutionelle Zusammenarbeit mit Kompetenzzentren für Geschlechterfragen in der Entwicklungszusammenarbeit:

„SDC implements therefore a gender mainstreaming approach by increasing the gender knowledge and awareness of staff and partners, the institutionalization of gender as a cross cutting issue and the promoting the gender responsiveness of all interventions."'(Siehe http://www.deza.admin.ch/de/Home/Projekte/Project_Detail?projectdbID=223793; Zugriff: 17.06.2014; siehe dazu auch Bieri 2006 und von Braunmühl 2001.)

Aus der Binnensicht einiger Befragter hat trotz dieser Bekenntnisse Gender Mainstreaming in der inhaltlichen Arbeit keinen großen Stellenwert mehr und ist nicht wirklich verankert.

Die Gleichstellungsbeauftragten von DEZA und EDA betonten im Gespräch, wie wichtig es sei, eine kohärente Politik zu machen - für das nach innen einzustehen, was man nach außen vertrete. In diesem Sinne ist eine umfassende betriebliche Gleichstellungspolitik „Ehrensache“. In der DEZA wurde 1997 ein Gleichstellungsplan mit einem Entwicklungshorizont bis 2010 verabschiedet; die aktuelle EDA-Strategie umfasst den Zeitraum 2010 2020. Als Ziel wurde formuliert, die Bedürfnisse und Anliegen von Frauen und Männern gleichermaßen zu berücksichtigen, und zwar in Programm, Organisation und Personal. Diese Vorstellung von Gleichstellung geht über eine Funktion der Personalpolitik hinaus und hat auch andere Wurzeln. Sie anerkennt, dass auch Organisationsstrukturen und Routinen vergeschlechtlicht sind. Gleichstellung wird explizit unter dem Aspekt der Organisationsentwicklung betrachtet, wie dies als Standard bei betrieblicher Gleichstellung gilt (Jüngling/Rastetter 2011: 32-35): 
„Echte Chancenförderung kann nur gelingen, wenn alle wesentlichen Dimensionen der Organisation (Identität, Konzepte und Strategien, Funktionen, Sachmittel, Prozesse, Menschen und Gruppen, Strukturen) in den Wandel einbezogen werden.“ (DEZA 1997: 3)

Die personalpolitischen Ziele sind ähnlich wie jene in der sonstigen Bundesverwaltung - nämlich paritätische Vertretung von Frauen und Männern, vertikale und horizontale Entwicklungsmöglichkeiten, Vereinbarkeit von Beruf und Familie -, werden aber um eine gleichstellungsfreundliche Unternehmenskultur ergänzt. Die Strategie des EDA von 2010 geht weiterhin von diesem umfassenderen Gleichstellungsverständnis:

„Wir verstehen den Prozess zur Förderung der Chancengleichheit als Teil eines umfassenden Organisations- und Kulturwandels, der alle Dimensionen der Institution betrifft. Im EDA positionieren wir Chancengleichheit als explizites Ziel und als bedeutenden Wert für unsere Organisation.“(EDA 2011: 5)

In der EDA-Strategie erscheint auch ein Diversity-Ansatz, indem die Vielfalt entlang von Geschlecht, Sprache, Nationalität und Alter für den Kompetenzzuwachs geschätzt wird. Diskontinuierlichere Berufsverläufe, die berufliche Situation von (ausländischen) Ehepartnern/Begleitpersonen und der Einbezug der familiären Situation vor Rotationen werden als Herausforderungen benannt; mit dem Anstieg des Frauenanteils in den oberen Hierarchiestufen, flexibleren Arbeitsarrangements und steigenden Arbeitsanforderungen wird die Hoffnung auf einen organisationskulturellen Wandel verbunden (EDA 2011).

\subsubsection{Gleichstellung durch Veränderungen der Institution}

Im Gegensatz zu den anderen Ämtern äußerten alle Befragten in der DEZA die Überzeugung, dass Gleichstellung eine Veränderung in der Institution bedinge, in Routinen, in der Organisationskultur und in besonderen Personalmanagementprozessen, da Geschlechterfragen ,überall“ präsent seien. Die Gleichstellungsbeauftragten erwähnten diese Triade der Gleichstellungsziele - nämlich Vereinbarkeit, mehr Frauen in Kaderpositionen und Transformation der Organisationskultur. In den weiteren untersuchten Ämtern war die Mehrheit hingegen der Meinung, Geschlecht irrelevant in der Organisation oder die Kultur sei bereits gleichstellungsfreundlich. In der DEZA wird Gleichstellung als sehr anspruchsvolle Aufgabe gesehen, die sich nicht von allein löst.

In diesem Zusammenhang wurde die Reorganisation mehrere Male erwähnt, die unter neuer Amtsleitung 2008 stattfand: Führungsstrukturen sollten gestrafft und die Arbeit stärker auf die operationale Tätigkeit auch im Terrain ausgerichtet werden. Dies folgte u.a. nach einem Bericht der ständerätlichen Geschäftsprüfungskommission, die bemängelt hatte, dass die Direktion sich verzettle. Bundesrätin Micheline Calmy-Rey sagte dazu in einem 
Interview, die DEZA sei stark gewachsen und habe wesentlich zum Ansehen der Schweiz in der Welt beigetragen, habe aber ein zu starkes „Eigenleben“ geführt. Sie müsse stärker mit anderen Abteilungen im EDA und mit dem SECO zusammenarbeiten (NZZ am Sonntag 2008). In diesem Rahmen wurden 400 Stellen bei insgesamt etwa 600 Beschäftigten mit einem veränderten Pflichtenheft neu ausgeschrieben, auf die sich die Mitarbeitenden neu bewerben mussten (Neue Zürcher Zeitung 2008). Die Gleichstellungsarbeit wurde für das gesamte EDA zentralisiert und deren Leitung von der damaligen DEZA-Beauftragten übernommen. Die Stabsstelle Chancengleichheit ist dabei sowohl von der DEZA als auch von anderen Direktionen des EDA getrennt im Stadtzentrum von Bern untergebracht. Die Nähe und Möglichkeiten $\mathrm{zu}$ informellen Kontakten werden einhellig vermisst. Zudem habe der Wechsel in der Amtsleitung dazu geführt, dass Chancengleichheit nicht mehr oben auf der Agenda stehe. Die Reorganisation zeige, dass der Frauenanteil nicht einfach automatisch steigt, obschon jetzt eine „Masse von Frauen“ von unten nachwächst.

„Bei den Restrukturierungsprozessen ist aus meiner Sicht nicht sehr gut gelaufen und wurde nie richtig aufgearbeitet: Es haben mehr weibliche Führungskräfte ihren Platz verloren als gewonnen. (...) Wenn man die Gesamtbilanz anschaut und man sagen muss, dass mehr Frauen aus Führungspositionen rausgefallen sind, dann muss man sich als Institution Fragen stellen. Natürlich bin ich schon lange dabei, kenne alle, kenne auch die Schwächen, ich kann es durchaus nachvollziehen, was die Begründungen waren, aber auf der anderer Seite gibt es Männer, die auch Schwachstellen haben und immer noch in Führungspositionen sind. (...) [I]ch würde sagen, das gleichstellungspolitische Gewissen hat keine große Rolle gespielt in dieser Umstrukturierung, oder es ging ein bisschen verloren." (Interview)

Einige Befragte wünschten eine stärkere Präsenz und Position der Gleichstellungsbeauftragten, die mit beratender Stimme in Auswahlkommissionen z.B. bei Rotationen dabei ist. Ihre Aufgabe ist es, auf Prozessgerechtigkeit zu achten, damit eben nicht bestimmte Geschlechterstereotype den Ausschlag für oder gegen eine Kandidatur geben: Wird bei allen gleich gefragt und werden Entscheidungskriterien gleich angewendet? Die beratende Stimme der Gleichstellungsbeauftragten führe dazu, dass auf ihre Einwendungen hin nochmals diskutiert und oft eine andere Entscheidung getroffen wird - ohne Vetorecht der Beauftragten.

Das Ziel der Vereinbarkeit und der guten Work-Life-Balance ist auch in der DEZA als Ziel prominent, allerdings wurden Modelle und Vorstellungen in ihren unterschiedlichen möglichen Auswirkungen reflektiert, was auf eine langjährige Auseinandersetzung mit dem Thema hindeutet.

„Wir müssen aufpassen, dass wir am Schluss nicht in ein früheres Modell zurückfallen: dass wir entweder Männer in klassischen Beziehungssituationen haben, wo die Frau einfach mitgeht; oder Frauen, die ihr Familienleben oder ihren Wunsch nach Kindern aufgegeben haben für ihre berufliche Karriere. In ganz frühen Tagen war das das Frauenbild der DEZA, das hat sich dramatisch geändert, ich würde sagen es sei Dank den Genderpolitiken. Wir haben viele Frauen, die Kinder haben, und auch Arbeit und Familie miteinander 
verbinden wollen, die bereit sind, mit dem Partner Kompromisse einzugehen, damit eben auch ein Auslandsaufenthalt möglich ist. Das sind aber sehr schwierige Gleichgewichte, die es zu suchen heißt, und wenn die Institution nicht hilft, Lösungen zu finden, ist es einfach noch schwieriger. Auch Männer sieht man immer mehr, die bereit sind, ihre Karriere so anzupassen, dass eben alles kombiniert werden kann. Auch da sollten es Lösungen geben, ich würde sagen, es wäre ein personalpolitisches Oberziel für die Gleichstellung, welches ganz entscheidend ist.“" (Interview)

Das Rotationsprinzip ist in der DEZA wie im diplomatischen Dienst ein wichtiges Merkmal der Berufslaufbahn. In der Entwicklungszusammenarbeit wird alle vier bis sechs Jahre der Arbeitsplatz gewechselt. In der Zentrale kann davon abgewichen werden, im Feld ist der Wechsel jedoch obligatorisch. Ein Verzicht auf eine Rotation kann sinnvoll sein, etwa bei Fachspezialisierungen, bedeutet aber einen Verzicht auf Karriereaufstieg. In der Rotation sei man auch auf informelle Netzwerke angewiesen. Eine zusätzliche Schwierigkeit ist genau die Frage, ob Partner oder Partnerin bereit ist, diese Rotation mitzumachen, denn für ihn oder sie ist eine Auslandsmission oft genug ein Bruch in der eigenen Laufbahn. Wenn das alte idealtypische Modell von Rotation mit einem männlichen Ernährer aufrechterhalten werde und einige Frauen es jetzt auch erfüllen können -, so gebe es kaum Lösungen für Double-Career-Paare oder Paare mit geteilter Verantwortung für die Kinder, so die Überlegungen der Beschäftigten. In dieser Denkweise ist Gleichstellung eben nicht durch Angleichung zu erreichen.

Auf der praktischen Seite versucht die Stabsstelle, Bedürfnisse von Begleitpersonen zu erheben und Maßnahmen zu entwickeln, um die Probleme abzufedern - seien es erleichterte Arbeitsbewilligungen, die Anerkennung von Ausbildungen oder Seminare. Neben den auch in anderen Ämtern präsenten Reflexionen über Mixité/Repräsentativität, Förderung der Sprachgruppen und Integration von Behinderten wird auch über die Organisationskultur nachgedacht:

„Und es sind so ganz banale Dinge, dass man denkt, Leute, die Teilzeit arbeiten, seien nicht wirklich interessiert an einer Karriere; oder die wollten gar nicht viel arbeiten. Und dann zeigt man durch stetes Thematisieren in verschiedenen Gremien auf, warum die Leute Teilzeit arbeiten, dass diese ihre Leistung durchaus bringen und es nicht so ist, als würden sie nicht arbeiten wollen oder keine Ambitionen haben. Oder die ganz banale Frage auch, wie gesprochen wird, also dass man die Kommunikation reflektiert.“ (Interview)

Als ein Erfolgsfaktor wurde von DEZA-Beschäftigten ein eindeutiges Bekenntnis der Führungsebene (Bundesrat und Amtsleitung) genannt. Wenn das oberste Management nicht eine klare Position habe und diese mit Überzeugung vertrete, werde es, diplomatisch ausgedrückt, „schwierig“. Weitere Faktoren sind eine langfristige Vision, Vernetzung und Durchhaltevermögen. Gleichstellung als Thema sei jedoch auf der Agenda „nach unten gerutscht“, Gender-Trainings wurden abgeschafft, einige Befragte sprachen von mehreren „Lücken“ bei der Personalpolitik. In der Gesamtschau wird daher die 
betriebliche und bei einigen auch die inhaltliche Gleichstellungspolitik als defizitär und unreflektiert bewertet. Auffällig ist insgesamt, dass in der DEZA am meisten über Geschlecht reflektiert wird, dieses Amt die längste und umfassendste Tradition betrieblicher Gleichstellungspolitik hat und insgesamt die Stimmen zur betrieblichen Politik am kritischsten ausfielen: Je mehr man über Geschlecht nachdenkt, desto umfassender und anspruchsvoller wird die Aufgabe der Gleichstellung wahrgenommen.

Der Fall der DEZA zeigt, dass eine lange Tradition von Gleichstellungspolitik und inhaltliche wie betriebliche Bestrebungen des Gender Mainstreamings Hand in Hand gehen mit einer hohen Sensibilität der Beschäftigten für Gleichstellungsfragen, mit einem hohen Reflexionsniveau in Bezug auf Ziele und unterschiedliche Gleichstellungsverständnisse sowie die Erfolgsbedingungen und Konsequenzen für die Organisation. Am Beispiel der Restrukturierung wird allerdings auch deutlich, wie sehr ein Nachlassen der Bemühungen unmittelbar Rückschritte zur Folge haben kann. Im Bereich der Gleichstellung ,rien n'est jamais acquis“ - nichts ist als dauerhaft und selbstverständlich erreicht anzusehen -, die Gleichstellung der Geschlechter bleibt ein umkämpftes Konzept.

\subsection{Fazit: Gleichstellungspolitischer Individualismus überwiegt}

Bei der Analyse der parlamentarische Geschäfte zur betrieblichen Gleichstellung und der Aussagen der verwaltungsinternen ExpertInnen wurde die zentrale Bedeutung der Anstellungsbedingungen klar: Vereinbarkeit und mehr Frauen in Führungspositionen sind ein gemeinsamer Nenner. Eine enge und gute Verzahnung mit der Personalpolitik ist daher wichtig. Während PolitikerInnen jedoch oft die Vorbildfunktion der Bundesverwaltung herausstreichen, wo Sinnvolles erprobt werden kann, ist die Vorbildwirkung in der Binnensicht nicht relevant; entsprechende Maßnahmen werden eher als Mittel zur Personalgewinnung verstanden (,,attraktiver Arbeitgeber"). In der Verwaltung werden die Maßnahmen insgesamt positiver als im Parlament bewertet.

Der Fokus auf Vereinbarkeit und Frauen in Kaderpositionen ist überwiegend implizit heteronormativ und elitenzentriert. Gleichstellung für weniger gut Qualifizierte wird gegenwärtig nicht mehr thematisiert. Eine integrierte Diversity-Perspektive - Geschlecht, Sprache, kultureller Hintergrund - lässt sich am ehesten im EDA-Programmdokument ausmachen. Im Bewusstsein der ExpertInnen ist ansonsten die Zielvorstellung einer möglichst hohen „Repräsentativität“ vor allem der Geschlechter und der Sprachregionen in der Bundesverwaltung anzutreffen. 
Die Annahme, gleichstellungsrelevante inhaltliche Arbeit erhöhe die Sensibilität und die Umsetzung betrieblicher Gleichstellungsmaßnahmen, muss präzisiert werden. Sie wird durch die klar unterschiedlichen Vorstellungen und Überlegungen zu Geschlecht in der DEZA gegenüber jenen in den anderen Ämtern bestätigt. Es zeigt sich aber auch, dass einige gleichstellungsrelevante Projekte innerhalb eines Amtes wie dem SECO nicht ausreichen, um das Nachdenken in der Organisation zu verändern, sondern offenbar die gesamte Arbeit dabei wichtig ist - und in der DEZA ist Gleichstellung seit über 15 Jahren immer wieder als Ziel der Entwicklungszusammenarbeit gefordert und gefördert worden. Sozialisation der langjährig Beschäftigten und viele Diskussionen scheinen dies befördert zu haben. Betriebliche Gleichstellungspolitik muss diese Bedingungen langfristiger organisationskultureller Veränderungen in ihre Programmatik einbeziehen.

Die Vorstellungen von der Gleichstellung der Geschlechter in der schweizerischen Bundesverwaltung zeigen in einigen Zügen Parallelen zu früheren Forschungsergebnissen. So dominieren universalistische und individualistische Sichtweisen, ähnlich wie es Andresen und Dölling für die Berliner Verwaltung beschrieben haben (Andresen/Dölling 2005: 180-185). Brigitte Liebig (2013) hat in einer Typologie vier Orientierungsmuster zu betrieblichen Gleichstellungskulturen in der Schweiz herausgearbeitet. Der von ihr beobachtete „männliche Traditionalismus“, der auf Homogenität in der Organisation und sexueller Differenz und mithin auf hegemonialer Männlichkeit beruht, konnte in der Bundesverwaltung nicht beobachtet werden. ${ }^{10}$ Auch ,betrieblicher Kollektivismus" mit einer De-Thematisierung betrieblicher Heterogenität und der Externalisierung des Gleichstellungsthemas kommt in der Bundesverwaltung nicht vor; allenfalls wird in vereinzelten Äußerungen, man sei im eigenen Amt schon sehr weit mit dem Thema, ein Widerstreben erkennbar, sich vertieft mit Gleichstellung auseinanderzusetzen. Der von Liebig identifizierte ,normative Individualismus“ ist auch Teil der dominanten Vorstellung von Geschlechtergleichstellung in der Bundesverwaltung; hier wird Gleichstellung auch, aber nicht nur als Ergebnis individueller Leistungen gesehen, sondern auch als Ergebnis individueller Entscheidungen bei entsprechend ausgebauten betrieblichen Rahmenbedingungen. Die Orientierungen in der DEZA gehen über individualistische Muster wie den ,pragmatischen Utilitarismus" hinaus. Sie fußen nämlich auf relativ intensiver Auseinandersetzung mit Geschlechterfragen und beziehen teilweise auch strukturelle Überlegungen mit ein. Dabei wird soziale Vielfalt und Variabilität anerkannt; diese Vorstellungen sind daher sowohl konstruktivistisch als auch strukturalistisch geprägt.

Vorstellungen von Geschlecht und Gleichstellung am Arbeitsplatz sind ganz offensichtlich in Bewegung und sie prägen organisationales Handeln

10 Einschränkend muss gesagt werden, dass Ämter mit überwiegend männlicher Belegschaft, etwa das Verteidigungsdepartement, nicht untersucht wurden. 
und die Organisationskultur. Auf der einen Seite stehen Überlegungen zur Transformation von männlich tradierten und konnotierten Beschäftigungsund Verfügbarkeitsnormen. Auf der anderen Seite wird aber ein radikaler Individualismus stärker: Wenn Frauen so sind wie Männer, Vereinbarkeitsmaßnahmen getroffen sind und Frauen sich an die strukturellen Rahmenbedingungen anpassen, dann klappt es auch mit der Gleichstellung (Jüngling/ Rastetter 2011: 30-32). Das Geschlechterverhältnis wird dabei nicht als ein gesellschaftliches Strukturverhältnis wahrgenommen, das sich eben nicht nur durch individuelle Entscheidungen verändern lässt. Weiterhin ließe sich schlussfolgern, dass im Zuge der Ökonomisierung der Gleichstellungsfrage die Gefahr besteht, dass die Forderung nach Angleichung an bestehende männliche Normen wieder die Oberhand gewinnt. Hieraus könnten aktualisierte Widerstände erwachsen, etwa zur Abschaffung von Quoten oder der Frauenförderung, weil doch beide Geschlechter gleich behandelt werden und scheinbar mit den gleichen Bildungs- und Laufbahnvoraussetzungen in der Organisation arbeiten. Auch dies müsste für eine wirksame betriebliche Gleichstellungspolitik bedacht werden. 


\section{Betriebliche Gleichstellungspolitik der Bundesverwaltungen im Ländervergleich}

\section{Silke Bothfeld, Gesine Fuchs, Andrea Leitner, Sophie Rouault}

Die Analyse der betrieblichen Gleichstellungspolitik in den Bundesverwaltungen der drei Länder hatte den Doppelcharakter der Bundesverwaltung zum Ausgangspunkt: Sie ist Gegenstand politischer Intervention in die Organisation hinein und muss Geschlechtergleichheit nach innen umsetzen. Gleichzeitig ist sie Urheberin politischer Aktivitäten, mit denen die Bundesverwaltung geschlechterpolitische Leitbilder und Modelle „guter Politik“ als Element von Policies im Sinne des Gender Mainstreamings nach außen transportiert. Daher stehen die Bundesverwaltungen unter besonderer Beobachtung im Hinblick auf die Gleichstellung ihrer Beschäftigten. Im Fokus dieser Analyse stand daher das Ensemble konkreter Steuerungsinstrumente (die Steuerungsregime) zur Gleichstellung der im Bundesdienst beschäftigten Frauen und Männer, mit denen der Gleichstellungsgrundsatz in den Organisationen implementiert werden soll. Als betriebliche Organisation ist dieses Steuerungsregime Teil eines größeren Policy-Regimes, das die drei großen Komplexe der allgemeinen Personalpolitik des öffentlichen Dienstes, die allgemeine Gleichstellungs- und Vereinbarkeitspolitik und die speziell auf den öffentlichen Dienst bzw. den Bundesdienst gerichtete Gleichstellungspolitik umfasst. Zudem interagiert sie mit den übergreifenden geltenden Normen und Regelungen des Geschlechterregimes (siehe Kapitel 2 in diesem Buch). In den vorstehenden Länderkapiteln haben wir daher untersucht, wie die Gleichstellungsinstrumente ausgestaltet sind, die in den Ressorts (Arbeit, Finanzen) in der Schweiz, Österreich und Deutschland auffindbar waren. Ein besonderer Fokus lag hier bei den Gleichstellungsbeauftragten, den Gleichstellungsplänen und -berichten als institutionelle Instrumente sowie den Vereinbarkeitsmaßnahmen und der Karriereförderung als substanzielle Maßnahmen. In diesem Kapitel geht es nun darum, die Steuerungsregime in den Bundesministerien der drei Länder systematisch zu vergleichen, um deren potenzielle Effektivität anhand unserer drei „Gütekriterien“ - Verbindlichkeit, Reichweite und Ausdifferenziertheit - einzuschätzen.

Um das Verständnis für die drei Varianzen der konservativen Geschlechterregime Österreichs, der Schweiz und Deutschlands zu schärfen, analysieren wir vergleichend die gleichstellungspolitischen Maßnahmen und ziehen Schlussfolgerungen für die Wirkungsweise von gleichstellungspolitischen Instrumenten. Es wird deutlich, was in den Länderfallstudien bereits anklang: Die Inkohärenz und Hybridität der drei konservativen Geschlechterregime spiegeln sich in den drei Steuerungsregimen wider. Zwar ist das gleichstellungspolitische Instrumentarium inzwischen vielfach recht weit ausdifferen- 
ziert und auf den ersten Blick weitgehend mit der allgemeinen Personalpolitik verknüpft, jedoch fehlt an vielen Stellen die Verbindlichkeit, damit es auch zur Anwendung gelangt. Unter welchen Bedingungen die Wirksamkeit der Instrumente verstärkt werden könnte, diskutieren wir im abschließenden Abschnitt.

\subsection{Bundesdienst - Enklave oder Vorreiter für die Beschäftigung hoch qualifizierter Frauen?}

Gleichstellung im Bundesdienst ist in allen drei Ländern bereits relativ lange gesetzlich verankert. Die für den Bundesdienst spezifischen Regelungen wurden zu Beginn der 1990er Jahre in Österreich (1993/2004), in der Schweiz (1991/2003) und auch in Deutschland (1994/2001) erlassen. Sie zielen einerseits darauf, durch konkrete Maßgaben günstige Bedingungen für die weiblichen Beschäftigten zu schaffen (z.B. durch familienfreundliche Arbeitszeiten), aber auch darauf, durch prozedurale Maßnahmen Einfluss auf die Verfahren der Personalpolitik in den Bundesministerien zu nehmen. Die gesetzlichen Regelungen in Deutschland und Österreich haben dabei eine stärkere Stellung als die schweizerischen Weisungen: Sie sehen verbindliche und konkrete Regelungen für die Bundesverwaltung vor, wie etwa die Einrichtung der Gleichstellungsbeauftragten oder die Berichtspflicht, aber auch eine explizite Gleichbehandlungsvorschrift für Teilzeitbeschäftigte (Deutschland). Im Unterschied zur Schweiz gehen in Deutschland und Österreich die spezifischen Regeln der Bundesgleichstellungsgesetze im Bereich der Vereinbarkeit über das Personalrecht hinaus und machen den öffentlichen Dienst des Bundes somit in beiden Ländern zu einem attraktiven Arbeitgeber für Eltern. Zudem schreiben die Gesetze die Gleichbehandlung bei der Besoldung und den Aufstiegsbeurteilungen vor - was in der Schweiz bereits im Personalrecht angelegt ist und durch die allgemeine Gleichstellungsgesetzgebung kontrollierbar gemacht wird. In allen drei Ländern sieht der rechtliche Rahmen vor, dass das leistungsbezogene Beurteilungswesen und die Aufstiegsverfahren Personen in Teilzeitarbeit nicht benachteiligen dürfen. Im Unterschied zu Deutschland soll die Einhaltung der Gleichbehandlungsvorschriften im österreichischen Bundesdienst von einer unabhängigen Bundesgleichbehandlungskommission überwacht werden, die durch das spezifische Gleichstellungsrecht für den Bundesdienst vorgeschrieben ist.

Die gesetzlichen Grundlagen sind in den drei Ländern somit durch unterschiedliche Prinzipien für die Verwirklichung der Gleichstellung der Geschlechter geprägt. In Deutschland und Österreich wird ein ausgeglichenes Geschlechterverhältnis in allen Funktionen durch gesetzliche Frauenfördergebote und Bevorzugungsregeln bzw. Quotierungsregeln für Bewerbungen 
und Gremienbesetzungen angestrebt. Entsprechende Regelungen sind - im Unterschied zur Schweiz - in den spezifischen Gleichstellungsgesetzen für den Bundesdienst enthalten. Die schweizerische Gesetzgebung folgt hingegen einem reaktiven Ansatz des Diskriminierungsverbots ohne einen expliziten Frauenförderansatz und ist dementsprechend auf das allgemeine Gleichstellungsgesetz und Bundespersonalgesetz beschränkt (siehe dazu ausführlich Kapitel 4 zur Schweiz). Das schweizerische Gleichstellungsrecht weist dafür - wie auch das US-amerikanische - stärkere rechtliche Instrumente zur Rechtsdurchsetzung (Verbandsklage, Untersuchungsgrundsatz) auf, die im deutschen und österreichischen Recht nicht zu finden sind.

In allen drei Ländern besteht der konservative Typus des modifizierten männlichen Ernährermodells fort, der auch das Policy-Regime zur beruflichen Gleichstellung und damit die geschlechterspezifischen Strukturen in allen Bereichen des Beschäftigungssystems prägt. Sowohl die sozialpolitischen Rahmenbedingungen (Elternfreistellungsregelungen) als auch die kulturellen Normen und Praktiken des Geschlechterverhältnisses reflektieren die fortbestehende Hauptverantwortung der Frauen für die Familien- und Betreuungsarbeit. Zwar hat die Erwerbsbeteiligung von Frauen über die vergangenen 20 Jahre an Bedeutung gewonnen, doch gibt es Varianzen innerhalb dieses Modells: In der Schweiz lag die Beschäftigungsquote 2013 bei den Frauen zwischen 15 und 64 Jahren mit 73,6\% um mehr als 5 Prozentpunkte über jener in Deutschland und Österreich, doch ist die Schweiz mit einer Teilzeitquote bei den Frauen von 61\% gemeinsam mit den Niederlanden im Ländervergleich der OECD führend, während Deutschland und Österreich „,nur“ im oberen Mittelfeld liegen. Gemessen in Vollzeitäquivalenten fällt somit das Volumen der Erwerbsbeteiligung in allen drei Ländern sehr ähnlich aus und variiert zwischen 52\% in Deutschland, 54\% in der Schweiz und 55\% in Österreich (OECD 2013b).

Dies zeigt deutlich, dass die schweizerischen Frauen die Vereinbarkeit von Familie und Beruf im hohen Maße über Teilzeitbeschäftigung mit einem recht geringen Stundenumfang realisieren (siehe Kapitel 4 und 7 zur Schweiz). Doch auch unter diesen Bedingungen erscheint die Vereinbarkeit schwierig, weil es gerade in der Schweiz an ausreichenden und bezahlbaren Angeboten außerfamiliärer Betreuung mangelt: Bei den Kindern zwischen drei und fünf Jahren lag der Anteil außerhäuslicher Betreuung 2012 bei nur $46 \%$, in Österreich immerhin bei $84 \%$ und in Deutschland bei $95 \%$. Von den jüngeren (unter drei Jahren) befanden sich $37 \%$ der schweizerischen Kinder in außerhäuslicher Betreuung, aber nur $19 \%$ in Österreich und $29 \%$ in Deutschland (OECD 2012). In der Schweiz ist die arbeitsrechtliche Elternfreistellung zudem auf die gesetzliche 14-wöchige Mutterschutzfrist plus zwei Wochen begrenzt, während in Deutschland und Österreich die Elternzeit bzw. Karenzregelungen bezahlte Freistellungsphasen von 12 bzw. 14 (Deutschland) oder 36 Monaten (Österreich) vorsehen. Erstaunlicherweise 
liegt die tatsächliche Erwerbstätigkeit der schweizerischen Mütter mit Kindern unter drei Jahren mit fast $60 \%$ deutlich über denen in Deutschland (40\%) und Österreich (30\%), wenn man die Freistellungsphasen berücksichtigt (OECD Family Database 2012). Teilzeitarbeit ist somit in allen drei Ländern, und in besonderem Maße in der Schweiz, zentral zur Realisierung von Erwerbstätigkeit überhaupt, sie gilt jedoch als wichtigstes Hindernis beim Zugang zu hoch qualifizierten Tätigkeitsbereichen.

Der öffentliche Dienst hat in allen drei Ländern eine große Attraktivität als Arbeitgeber für Frauen: Trotz des über die 2000er Jahre fortgesetzten Beschäftigungsrückgangs in den öffentlichen Diensten insgesamt (auf 10\% bis $12 \%$ der Gesamtbeschäftigung in den drei hier untersuchten Ländern) ist der Frauenanteil im öffentlichen Dienst kontinuierlich gestiegen und erreicht mindestens den Anteil an der Gesamtbeschäftigung (Tab. 8.1). Im Bundesdienst sind die Frauen jedoch nach wie vor unterrepräsentiert: So liegt der Frauenanteil 2011/12 in Deutschland bei 25\%, in der Schweiz bei 32\% und in Österreich bei 41\% (ohne LehrerInnen 33\%, siehe Kapitel 6 zu Österreich). Die Unterschiede zwischen den Ländern sind teilweise auf die unterschiedliche Abgrenzung des Bundesdienstes von Kommunalaufgaben und damit auf seine unterschiedliche Tätigkeitsstruktur zurückzuführen und lassen somit noch keine gleichstellungsrelevanten Aussagen zu (siehe Kapitel 3 in diesem Buch). Betrachtet man den allgemeinen Verwaltungsdienst und rechnet die Verteidigung, die öffentliche Sicherheit, den Bundesgerichtshof, die Staatsanwaltschaft und den Schuldienst heraus, so liegen die Frauenanteile wiederum bei $41 \%$ für Deutschland und $45 \%$ für die Schweiz, und Österreich liegt mit 53\% vorn. Im Bereich der obersten (politischen) Staatsverwaltung, den obersten Bundesbehörden, erreichen Frauen fast den Anteil des gesamten öffentlichen Dienstes und der Gesamtwirtschaft.

Die eigentliche Ungleichheit zwischen den Geschlechtern im politischen Verwaltungsdienst des Bundes zeigt sich erst bei der Betrachtung der Führungspositionen in den Bundesministerien, des Anteils der Teilzeitbeschäftigten und der geschlechterspezifischen Einkommensdifferenzen. In der Schweiz liegt der Frauenanteil bei den Top-Führungspositionen und im mittleren Management deutlich auf dem letzten Platz des Drei-Länder-Vergleichs (10\% bzw. 12\%). Dementsprechend ist auch die geschlechterspezifische Einkommenslücke zwischen Frauen im Bundesdienst mit 12\% sehr hoch und gemessen an der Gesamtwirtschaft (18\%) immer noch beträchtlich (vgl. Strub/Stocker 2010). Deutschland weist zwar den insgesamt geringsten Frauenanteil im Verwaltungsdienst des Bundes auf, allerdings haben hier Frauen 2010 in den höchsten und mittleren Führungsebenen einen Anteil von etwa 20\% erreicht; Geschlechterunterschiede beim Einkommen sind hingegen nicht nachgewiesen. Auch in Österreich ist der Frauenanteil im Verwaltungsdienst am höchsten, und der Bundesdienst weist zugleich recht hohe Frauenanteile in den höchsten und mittleren Führungsebenen auf (23\% bzw. 36\%). 
Gleichzeitig ist der Verdienstunterschied zwischen den Frauen und Männern im gesamten Bundesdienst im Jahr 2011 mit 15\% am höchsten, vermutlich aufgrund des hohen Frauenanteils im Sekretariatsbereich (siehe Kapitel $6 \mathrm{zu}$ Österreich).

Tab. 8.1: Frauenbeschäftigung der Bundesdienste und in der Gesamtwirtschaft im Ländervergleich - zentrale Indikatoren (in \%)

\begin{tabular}{|c|c|c|c|}
\hline & $\mathrm{CH}$ & D & Ö \\
\hline Beschäftigungsquote Frauen (2013, EU) & 78,0 & 69,0 & 68,0 \\
\hline Frauenanteil Gesamtwirtschaft (2013) & 45,1 & 46,0 & 46,0 \\
\hline Frauenanteil öffentlicher Dienst (2013) & 45,5 & 55,1 & 45,1 \\
\hline Frauenanteil Beschäftigte im Bundesdienst (2011/12) & 31,6 & 25,1 & $40,6(33,0)^{*}$ \\
\hline $\begin{array}{l}\text { Frauenanteile Beschäftigte im Bundesdienst/nur Verwaltungs- } \\
\text { dienst }(2011 / 12)\end{array}$ & 45,0 & 41,0 & 53,0 \\
\hline $\begin{array}{l}\text { Frauenanteile Beschäftigte in den obersten Bundesbehörden } \\
(2011 / 2012)\end{array}$ & n.v. & 51,0 & 50,0 \\
\hline $\begin{array}{l}\text { Frauenanteil oberste Führungsebene des Bundesdienstes } \\
\text { (2010) }\end{array}$ & 10,4 & 19,5 & 22,6 \\
\hline Frauenanteil mittlere Führungsebene des Bundesdienstes (2010) & 12,0 & 19,6 & 35,9 \\
\hline Teilzeitquote Frauen Gesamtwirtschaft (EU, 2013) & 61,0 & 47,0 & 46,0 \\
\hline Teilzeitquote Frauen Bundesdienst insgesamt (D + Ö 2013) & 47,8 & 31,4 & 33,0 \\
\hline Gender Pay Gap Gesamtwirtschaft (2013) & 18,9 & 22,0 & 23,4 \\
\hline Gender Pay Gap Bundesdienst (2011, CH 2010) & 12,1 & 1,0 & 15,0 \\
\hline
\end{tabular}

* ohne Lehrpersonal

Quelle: Zusammenstellung der Autorinnen nach den jeweiligen Bundesstellen für Statistik

Bei der Teilzeitarbeit zeigen sich zwei interessante Befunde: Zum einen wird in den Bundesdiensten aller drei Länder wesentlich weniger Teilzeit gearbeitet als in der Gesamtwirtschaft. Dies illustriert nochmals die Tatsache, dass der Bundesdienst in seiner Tradition in allen drei Ländern männlich geprägt ist und Teilzeitarbeit in diesem Bereich erst spät ermöglicht wurde. Interessant ist allerdings, dass die Frauen im Bundesdienst der Schweiz eine Teilzeitquote von immerhin $48 \%$ aufweisen, während die Teilzeitquote bei den weiblichen Beschäftigten im Bundesdienst in Deutschland und in Österreich bei nur etwa einem Drittel liegt. Möglicherweise ist es die Teilzeitarbeit in der Schweizerischen Bundesverwaltung, die berufliche Fortentwicklung und die Aufstiegschancen für Frauen in Führungspositionen erschwert. Dieses Phänomen ist vermutlich jedoch ebenso in Österreich und Deutschland relevant.

Im Gesamtvergleich sind zwar alle drei Länder dem konservativen Geschlechtermodell zuzurechnen, die Schweiz hebt sich aber dadurch von Deutschland und Österreich ab, dass Vereinbarkeit weitgehend individualisiert über Teilzeitbeschäftigung organisiert ist und die „Folgekosten“ indivi- 
duell von den Frauen getragen werden. Die Stärkung der Teilzeitstrategie unterstützt einen breiteren Zugang zum Bundesdienst, schränkt allerdings die Karrierechancen bei Arbeitszeitreduktion erheblich ein. Für alle drei Länder zeigt sich somit, dass in einer besseren Anerkennung und Integration von Teilzeitbeschäftigung nur eine Teillösung für die berufliche Gleichstellung liegen kann.

\subsection{Substanzielle Maßnahmen der Gleichstellungsförderung: Vereinbarkeit und Karriereförderung}

\subsubsection{Modernisierte Personalpolitik: Vereinbarkeit von Beruf und Familie im Bundesdienst}

Der größte Vorteil der Beschäftigung im öffentlichen Dienst ist ohne Frage die Beschäftigungssicherheit, die gerade für Eltern bzw. Mütter mit familiär bedingten Unterbrechungen attraktiv ist. Hinzu kommt die „Familienfreundlichkeit" der Personalpolitik des öffentlichen Dienstes, die sich die Vereinbarkeit von Beruf und Familie zur Aufgabe macht und einen besonderen Schutz von Teilzeitbeschäftigten einschließt. Durch die Gleichstellungsregelungen in der Bundesverwaltung werden zusätzlich Gleichstellungspläne eingefordert, in denen die einzelnen Bundesministerien Maßnahmen wie etwa flexible Arbeitszeitgestaltung, Kinderbetreuung und zum Teil Beurlaubungen konkretisieren müssen. Über die Weiterentwicklung der Maßnahmen zur Vereinbarkeit besteht ein breiter Konsens zwischen Führungskräften und Beschäftigten, da diese als Verbesserung der Beschäftigungsbedingungen insgesamt und weniger als ein Verteilungskonflikt zwischen den Beschäftigtengruppen (hier: Frauen und Männer) betrachtet werden. Eine explizite politische Zielstellung, die Beteiligung der Männer an der Familienarbeit zu unterstützen („Papamonate“), zeichnet sich seit 2008 in Österreich und in Deutschland etwas später ganz deutlich ab. Die drei Maßnahmenbündel zu Teilzeitarbeit, Beurlaubung und Kinderbetreuung, die Bundesbeschäftigten zur Verfügung stehen, verhalten sich weitgehend komplementär und richten sich, wenngleich sie geschlechterneutral formuliert sind, in der Praxis jedoch überwiegend an Frauen.

\section{Teilzeitarbeit im Bundesdienst}

Die Regelungen zur Teilzeitarbeit, die BeamtInnen erst spät ermöglicht wurde (Deutschland 1969, Österreich 1979, Schweiz 1986) sowie die Möglichkeiten zur Telearbeit sind in den drei Ländern sukzessive ausgeweitet worden 
mit dem Ziel, die Vereinbarkeit von Beruf und Familie zu verbessern. In der Schweiz besteht überraschender Weise erst seit 2013 ein Rechtsanspruch auf Teilzeitarbeit, allerdings nur für Eltern im Anschluss an die Mutterschutzfrist und mit einer Begrenzung der möglichen Reduzierung auf mindestens $60 \%$ einer Vollzeitstelle. In der Schweiz ist für die fortgesetzte Entwicklung der Teilzeitarbeit vor allem der Wandel von Normen und gelebten Werten ursächlich, denn Teilzeitarbeit war nur ganz allgemein als eine Möglichkeit im Personalgesetz geregelt und auf individuelle Vereinbarungen zwischen Beschäftigten und Vorgesetzten angewiesen. Dies tat ihrer Verbreitung keinen Abbruch, auch wurde nur von wenigen Konfliktfällen berichtet. Insofern erscheinen ,ein gezielter organisationskultureller Wandel“" (so ein Interview in der DEZA) bzw. die schlichte Notwendigkeit, Müttern eine Teilzeittätigkeit zu gewähren, als die eigentlichen Triebkräfte für die Verbesserung der Vereinbarkeit von Beruf und Familie und nicht ein gesetzlicher Anspruch.

In Deutschland besteht laut Teilzeit- und Befristungsgesetz ( $\$ 8 \mathrm{TzBfG})$ ein Rechtsanspruch auf Teilzeitarbeit für alle Beschäftigten, der gemäß dem Gleichstellungsgesetz bei Elternschaft nur aufgrund ,Zwingender betrieblicher Gründe" abgelehnt werden kann, was dann schriftlich zu begründen ist. In Österreich besteht ebenfalls ein Rechtsanspruch auf Teilzeitarbeit, die bei BeamtInnen (wie in Deutschland bei BeamtInnen ohne Kinder) mindestens jedoch die Hälfte der regulären Wochenarbeitszeit betragen muss, bei Angestellten jedoch auch darunter liegen kann. Die Rückkehr zur Vollzeitarbeit ist vor Ablauf der beantragten Dauer nicht eindeutig geregelt, jedoch soll zumindest in Deutschland laut $\S 9$ TzBfG Teilzeitbeschäftigten Vorrang bei der Rückkehr zu Vollzeitarbeit gewährt werden. In der Praxis werden die Probleme von Teilzeitarbeit offenbar: Das Problem der Überforderung und Arbeitsintensität ist nicht immer gelöst, weil wegfallende Stellenanteile nicht systematisch in den Arbeitseinheiten ersetzt werden; zudem werden an die Arbeitskontexte höhere Anforderungen an Kooperationsfähigkeit und Informationsprozesse gestellt (siehe Kapitel 6 zu Österreich). Folglich ist die Gewährung von Teilzeitarbeit für Referats- oder ArbeitsgruppenleiterInnen nicht immer attraktiv, weil die Arbeitsprozesse schwieriger zu organisieren sind, während die Menge der zugeordneten Aufgaben sich nicht reduziert. Schließlich verfestigen sich geschlechtsspezifische Arbeitsteilungsmuster und Karrierenachteile für die Teilzeitbeschäftigten (ExpertInneninterview Schweiz, Deutschland). Für Deutschland sind die Nachteile durch Teilzeit bei der Laufbahnentwicklung eindrucksvoll belegt: Hier ist die Beförderungsquote von Vollzeitkräften 2,3-mal höher als jene von Teilzeitbeschäftigten (siehe Kapitel $5 \mathrm{zu}$ Deutschland). Aber auch in der Schweiz ist die Chance für Frauen, zur Führungskraft aufzusteigen, vergleichsweise gering; möglicherweise auch aufgrund des hohen Teilzeitanteils bei Frauen. Insgesamt befördert das Anwachsen der Teilzeit- und der Telearbeit jedoch eine Schwächung von Verfügbarkeits- und Anwesenheitskulturen in den Bundesministerien, 
besonders sichtbar in Deutschland und der Schweiz, wo die Teilzeitquote auch in höheren Positionen angestiegen ist. In der Schweiz ist zudem das Vertrauen in den „kulturellen Wandel durch Technologie“ bei Telearbeit durchgehend hoch.

\section{Beurlaubungen für Elternschaft im Bundesdienst}

In Deutschland und Österreich orientieren sich die Elternfreistellungsregelungen für den Bundesdienst - auch bei den BeamtInnen - an den allgemeinen für alle Beschäftigten geltenden arbeitsrechtlichen Regelungen. Allein im deutschen Beamtenrecht gibt es die Möglichkeit der Freistellung bis zu einer Dauer von insgesamt zwölf Jahren. Im Unterschied zur Privatwirtschaft sieht das österreichische Personalrecht den Frühkarenzurlaub für Väter („Papamonat") vor, der eine vierwöchige bezahlte Freistellung für Väter nach der Geburt eines Kindes vorsieht, der auch während des Beschäftigungsverbots der Mütter genommen werden darf. In Deutschland bietet die allgemeine Regelung der Vätermonate einen besonderen Anreiz für alle Väter, wenigstens zwei Monate der bezahlten Freistellung zu beanspruchen; mit der neuen Regelung zum „Elterngeld plus“ (ab 1. Januar 2015) ergeben sich für Eltern zusätzlich flexible Möglichkeiten zur Vereinbarung von Elterngeld mit einer Teilzeitarbeit; spezifische Regelungen für Bundesbedienstete gibt es hingegen nicht. In Deutschland hat sich dementsprechend im Allgemeinen der Anteil der Väter, die die Freistellungen beanspruchten, sprunghaft erhöht: Bei den 2011 geborenen Kinder nahmen bereits 27,3\% der Väter zumindest einen Teil der Elternzeit (gegenüber 19,2\% bei Vätern von im Jahr 2008 Geborenen); in Österreich war der Anteil der Väter mit 14\% noch geringer.

Die negativen Folgen der durch die langen Elternkarenzmöglichkeiten bedingten Berufsdiskontinuitäten sind im Bundesdienst aufgrund der höheren Arbeitsplatzsicherheit weniger gravierend als in der Privatwirtschaft, jedoch beruflichen Aufstiegen ebenso abträglich. Daher werden sie vor allem in den Frauenförderplänen nachdrücklich thematisiert. So wird etwa in Österreich den negativen Folgen für die Berufslaufbahn mit Maßnahmen zur Information und Einbeziehung von Eltern während der Karenz bei Besprechungen, Beratungsangeboten für Karenz und Wiedereinstieg sowie Qualifizierungsangeboten für WiedereinsteigerInnen präventiv begegnet. In Deutschland sieht das Gleichstellungsgesetz für den Bundesdienst ähnliche Maßnahmen vor, die die Wiedereingliederung von Eltern erleichtern sollen. Die Laufbahnstrukturen des öffentlichen Dienstes sind hier zwar offener und flexibler geworden, sodass bei der Beurteilung und Beförderung Unterbrechungen keine Rolle spielen sollen. Jedoch können sachliche Gründe angeführt werden, um eine Ungleichbehandlung zu rechtfertigen (s.u.). In der Schweiz ist die Situation deutlich anders: Zwar sieht das Personalrecht eine etwas längere Mutterschutzfrist vor (16 statt 14 Wochen), darüber hinaus gibt es aber keine arbeitsrechtliche Freistellung für Eltern. Eine freiwillige Beurlaubung ist 
zwar möglich, wird jedoch selten beansprucht. Da die Frauen nach der Mutterschutzfrist in der Regel eine kurzfristige Teilzeitarbeit aufnehmen, ist der Wiedereinstieg von Eltern in der Bundesverwaltung, anders als in den 1990er Jahren, bei den betrieblichen Maßnahmen kein Thema mehr. Hier bildet sich vielmehr eine Norm der kontinuierlichen Erwerbstätigkeit heraus (vgl. Kapitel 4 und 7 zur Schweiz).

\section{Kinderbetreuung für Eltern im Bundesdienst}

Die Situation der Kinderbetreuung ist für die Beschäftigten der Bundesverwaltung insgesamt günstig, weil die Bundesverwaltungen in den Großstädten angesiedelt sind, wo in der Regel ein größeres Angebot an Kinderbetreuungseinrichtungen vorhanden ist. In Deutschland gibt es keine zusätzlichen Maßnahmen oder eigene Einrichtungen für die Betreuung von Kindern, da Kinderbetreuung durch den allgemeinen Rechtsanspruch (seit August 2013) auf einen Betreuungsplatz geregelt sein sollte. Von österreichischen ExpertInnen werden die Vorteile qualitativ hochstehender betrieblicher Kinderbetreuung betont; es bestehen Betreuungseinrichtungen für Kinder von Bundesbediensteten, die aber nur einen geringen Anteil der Kinderbetreuung abdecken. In der schweizerischen Bundesverwaltung sind eigene Kinderbetreuungseinrichtungen abgebaut worden, doch wurden Subventionen für die vorschulische Kinderbetreuung eingeführt. Sie sind relativ gesehen wichtiger als in den anderen Ländern, weil in der Schweiz der größte Anteil der Betreuungskosten privat getragen wird. Mit dieser Maßnahme geht man über die sonst eher geringen Bemühungen, Vereinbarkeit öffentlich zu fördern, hinaus.

$* * *$

Ohne Zweifel gilt für alle drei Länder, dass die traditionelle Anwesenheitskultur in den Bundesverwaltungen durch das allgemeine klare Bekenntnis zur Vereinbarkeit von Beruf und Familie allmählich geschwächt wird. In Deutschland sind Vereinbarkeitsmaßnahmen mit den - im Vergleich zur Privatwirtschaft etwas stärkeren - Rechtsansprüchen (vor allem auf Teilzeitund Telearbeit) im höheren Maße verbindlich geregelt. In Österreich helfen die Gleichstellungspläne, konkrete Maßnahmen als „,fremdes“ Element in die Personalpolitik einzubringen. In der Schweiz besteht nur ein selektiver Rechtsanspruch auf Teilzeitarbeit, hier ist es vielmehr der allgemeine Wandel von gesellschaftlichen Normen, der zur Verbreitung von Teilzeitarbeit geführt hat.

Am weitesten ausdifferenziert sind die Maßnahmen zur Vereinbarkeit in Österreich: Hier reduzierte die Förderung der Wiedereingliederung und die Einbeziehung der Väter die Hauptlast der familienbedingten Erwerbsunterbrechung, die zuvor allein durch die Mütter getragen wurde. In der Schweiz 
hingegen wird vorwiegend auf private Lösungen auf Basis von Teilzeitarbeit gesetzt.

In den anderen beiden Ländern sorgt hingegen die prinzipiell gute Koordination mit allgemeinen Vereinbarkeitsmaßnahmen (Vätermonate, öffentliche Kindertagesbetreuung) für eine gute Reichweite der Vereinbarkeitspolitik, die wenig selektierende Effekte bei den Beschäftigten erwarten lässt. Interessant und ein Potenzial für zukünftige Entwicklungen ist ohne Frage die Teilzeitarbeit, die in allen drei Bundesverwaltungen deutlich unter dem Gesamtdurchschnitt liegt. Hier wäre vor allem zu klären, wie der Umgang mit teilzeitig besetzen Stellen verbessert werden kann, sodass wegfallende Stellenprozente nicht zu Lasten des konkreten Arbeitskontextes und der Teilzeitbeschäftigten gehen.

\subsubsection{Förderung von Frauen in Führungspositionen: Wie sind direkte und indirekte Diskriminierung zu vermindern?}

In den Bundesverwaltungen aller drei Länder war die Überzeugung wahrnehmbar, der demografische Wandel mit seinem Mangel an qualifizierten Arbeitskräften und die wachsende Anzahl gut ausgebildeter junger Frauen würden über die Zeit automatisch zu einer ausgeglichenen Repräsentation der Geschlechter auch in den Bundesverwaltungen beitragen.

Dem stehen jedoch die noch existierenden beamtenrechtlichen Regulierungen entgegen, die sich aus der Grundidee des Beamtentums mit seinen lebenslangen Karrieren ableitet, nach der weibliche Erwerbsverläufe mit der ganzheitlichen Dienstauffassung des Beamtentums nicht kompatibel sind. Zwar wurden direkte Diskriminierungen beseitigt, doch bestehen etwa in der deutschen Bundesverwaltung strukturelle Gründe für die Ungleichheit bei der Rekrutierung und dem beruflichen Aufstieg von Frauen und Männern fort. Anders als in der Vereinbarkeitspolitik lassen die gleichstellungspolitischen Anforderungen ein Spannungsfeld entstehen: Zum einen wird die stärkere Berücksichtigung von Frauen als ein Verteilungskonflikt zu Lasten der männlichen Beschäftigten empfunden und die „automatische“ Bevorzugung von Frauen kritisiert und rechtlich beanstandet. ${ }^{1}$ Zum anderen müssen die „sachlichen Gründe“, die bei der Beurteilung der Qualifikation und Eignungsfeststellung von BewerberInnen zugrunde gelegt werden, geprüft werden, ob sie wirklich diskriminierungsfrei sind. Die ,sachlichen Gründe“ sollten also nicht auf Kriterien und Kompetenzen abstellen, die eher von Männern erbracht werden (können), die aber für die Eignung und Qualifikation keine Rolle spielen. Daher ist es für die Gleichstellung von Frauen und Män-

1 Wie es das grundlegende Urteil des Europäischen Gerichtshofs vom 17.10.1995 (Kalanke, C-450/93) illustriert. Ein Beamter der bremischen Verwaltung hatte dies Verfahren gegen die Bevorzugung einer weiblichen Kandidatin eingeleitet. 
nern besonders wichtig, die Verfahren der Rekrutierung und Leistungsbeurteilung durch klare Beurteilungskriterien und Prozessabläufe transparent und objektiv zu gestalten.

\section{Rekrutierung von neuen Bundesbediensteten}

In Österreich legt das Gleichbehandlungsgesetz für die Bundesverwaltung klare Quoten- und Bevorzugungsregeln in Kombination mit der Berichtspflicht als ein zentrales Instrumentarium für die gezielte Rekrutierung von Frauen fest. Tatsächlich unterstützen die Quotenregelungen die Arbeit der Gleichbehandlungsbeauftragten, weil sie in den Verfahren für Transparenz und Nachvollziehbarkeit der Verwaltungsabläufe sorgen. So muss entsprechend dem Gesetz die Passung zwischen den Qualifikationen der BewerberInnen und den Anforderungen der offenen Stelle fachlich bewertet und standardisiert protokolliert werden und einen fundierten Vergleich erlauben. Allerdings zeigt die Praxis, dass der Begriff der Qualifikation in vielen Fällen zu unspezifisch ist, sodass die Argumente der Gleichstellungsbeauftragten für die Rekrutierung von Frauen leicht ausgehebelt werden können. Ähnliches gilt für den deutschen Bundesdienst, wo das Kriterium der ,gleichen Qualifikation" oftmals nicht eindeutig zu beurteilen ist: Hier wird zuweilen die Feststellung der Leistungsmerkmale der BewerberInnen durch eine wenig sinnhafte Ausdifferenzierung konterkariert, um die Feststellung „gleicher Eignung, Befähigung und fachlicher Leistung" ( $\$ 8$ BGleiG) zu vermeiden und die Auswahl eines Kandidaten zu begründen (siehe Kapitel 5 zu Deutschland). Zudem fehlen die institutionellen Rahmenbedingungen und Ressourcen für die Implementation von Quotenregelungen: So sind weder in Deutschland noch in Österreich (Zwischen-)Ziele oder Zeitrahmen und auch keine Mechanismen für die Überprüfung ihrer Anwendung (z.B. ein parlamentarischer Kontrollmechanismus) festgelegt. In der Schweiz gibt es zwar lediglich eine wenig verbindliche „Soll-Vorgabe“, nach der das unterrepräsentierte Geschlecht stärker berücksichtigt werden soll. Allerdings sieht die schweizerische Personalstrategie klar bezifferten Zielvorgaben für die Frauenanteile in den verschiedenen Lohnklassen vor, die auch im Rahmen der Berichtspflicht regelmäßig überprüft werden. Davon abgesehen gibt es Einzelfälle, in denen sich die BundesrätInnen oder Vorgesetzten bei Einstellungs- und Beförderungsverfahren besonders für die Rekrutierung von Frauen einsetzen (siehe Kapitel 4 und 7 zur Schweiz). Insgesamt zeigt sich, dass, je klarer das konkrete Vorgehen und die Berichtspflicht in den verschiedenen Verwaltungen geregelt sind, Zielformulierungen und Quoten eher wirksam werden können. Von diesen formalisierten Instrumenten abgesehen, ist die gezielte Ansprache von qualifizierten Frauen durch Gleichstellungsbeauftragte oder engagierte Vorgesetzte, sich auf ausgeschriebene Führungspositionen $\mathrm{zu}$ bewerben, in allen Ländern eine Routinemethode der Frauenförderung 
(Interviews), die jedoch nicht standardmäßig angewandt wird und auch nicht formal vorgeschrieben ist.

Verfahren der Leistungsbeurteilungen und Karriereförderung

Gute Leistungsbeurteilungen bilden in allen drei Ländern die Voraussetzung für den Aufstieg in Führungspositionen und die Gewährung von Prämien und Zulagen. Im öffentlichen Dienst und insbesondere bei den BeamtInnen des Bundesdienstes sind die Leistungsbeurteilungen besonders genormt und standardisiert und besonders anfällig für geschlechterspezifische Diskriminierung (Krell 2011). Die in den drei Ländern interviewten ExpertInnen schätzen das Diskriminierungspotenzial der internen Leistungsbeurteilungen jedoch sehr unterschiedlich ein. In der Schweiz und Deutschland wurde in Anerkenntnis des Geschlechterbias die Berücksichtigung von ,außerberuflichen Erfahrungen“ wie Betreuungsaufgaben oder soziales Engagement festgeschrieben, deren faktische Anwendung jedoch unklar ist. In der Schweiz sticht jedoch das Prinzip der internen Veröffentlichung der Geschlechterverteilung von Prämien und Beurteilungen hervor und macht deutlich, wie wirkungsvoll das Instrument des Benchmarking auch ohne materielle Sanktionsdrohung sein kann, da sich die geschlechterspezifischen Unterschiede tatsächlich verringern. In Deutschland wird das Verfahren der Leistungsbeurteilung hingegen als besonders problematisch beurteilt, weil das Beamtenrecht mit seiner starren Laufbahnorientierung die Möglichkeiten der Karriereförderung stark vorstrukturiert. Dabei ist die Überarbeitung der Leistungskriterien ebenso umstritten wie langwierig. Zudem produziert die in der deutschen Bundesverwaltung praktizierte Kontingentierung der Beurteilungsstufen Verteilungskonflikte, die eine gleichstellungspolitische Korrektur zusätzlich erschweren (vgl. Kapitel 5 zu Deutschland). In Österreich wird das potenzielle Problem eines Geschlechterbias beim Verfahren zur Leistungsbeurteilung hingegen nicht thematisiert; vielmehr setzt die österreichische Bundesverwaltung einen starken Fokus auf die Beteiligung von Frauen an Weiterbildungsveranstaltungen. Dies geschieht vor allem durch die bevorzugte Vermittlung von Frauen in Weiterbildungen, aber auch durch ein spezifisches Kursangebot zu Karriere und Laufbahn. Zusätzlich gibt es Angebote für Mentoring, Coaching oder Supervision. In den anderen beiden Ländern gibt es ähnliche Programme, wobei in einem schweizerischen Departement auch die Möglichkeit der „Potenzialanalyse“ angeboten wird. In der Schweiz sind die Maßnahmen teilweise in ein Programm eingebettet, das auch Vereinbarkeitsmaßnahmen umfasst und aus dem Beschäftigte bzw. ihre Vorgesetzten das passende Instrument auswählen können. In Österreich gibt es weitere Ziele, etwa für geschlechtergerechte Unterrichtsmaterialien in der Weiterbildung. Gemeinsam ist der schweizerischen und österreichischen Bundesverwaltung, dass der Fokus bei der Weiterbildung faktisch auf höher Qualifizier- 
ten liegt, während im deutschen Bundesdienst mehr Wert auf einen breiten und individuellen Zugang zu Weiterbildung gelegt wird.

$* * *$

Insgesamt ist der Einsatz von Maßnahmen zur Karriereförderung - Quotenregelungen und Rekrutierungsmechanismen, Leistungsbeurteilungen, Weiterbildung und individuelle Förderung - zwischen den einzelnen Ministerien bzw. Ämtern sehr heterogen und deren Anwendung stark abhängig von den internen Organisationskulturen. Dabei unterscheiden sich die deutschen und österreichischen Regeln von ihrem schweizerischen Pendant durch gesetzliche Quotenbestimmungen für Rekrutierung und Beförderung, die in höherem Maße verbindlich sind. Die Verbindlichkeit der deutschen und der österreichischen Regulierung wird jedoch durch die fehlende Ausdifferenzierung der Ziele und Zwischenziele sowie eine klare Definition des Qualifikationsbegriffs unterlaufen. In der Schweiz hingegen ist die Bevorzugungsregelung weniger verbindlich, aber es wird differenziert nachgehalten: Hier kommen die weitreichenden und ausdifferenzierten Implementationsregeln und -ressourcen (Planpflicht, Kontrollfunktion der Gleichstellungsbeauftragten) zum Tragen, durch die die Zielvorschriften erst in die Praxis gelangen. Grundsätzlich gilt aber auch, dass die Instrumente zur Laufbahnentwicklung in allen drei Verwaltungen vor allem bei den Hochqualifizierten ansetzen. Besonders in der Schweiz sind sie auf die Förderung von Frauen in Führungsfunktionen verengt, im Gegensatz zu Österreich, wo auch explizit Frauen mit geringeren Qualifikationen auf Weiterbildungen angesprochen werden. Allerdings kommen durch die Regeln für die Rekrutierung wiederum die Arbeitsbedingungen, und somit vor allem die Vereinbarkeit von Beruf und Familie, als Gegenstand der Reflexion auf die Agenda. Insofern ist eine entscheidende Frage, inwiefern bei der Rekrutierung von (jungen) Frauen, die weniger mit dem Berufsbeamtentum kompatible Erwerbsverläufe aufweisen, tatsächlich Erfolge erzielt werden können. Insofern bilden Maßnahmen zur Vereinbarkeit und zur Karriereförderung zwei komplementäre, bislang jedoch gleichzeitig widerläufige Instrumente einer umfassenden und effektiven Strategie der Gleichstellungsförderung.

\subsection{Institutionelle Maßnahmen für mehr Geschlechtergerechtigkeit in der Personalpolitik der Bundesverwaltung}

Während die substanziellen Maßnahmen weitgehend über personalrechtliche Vorschriften institutionalisiert und durch die Gleichstellungsgesetze bzw. -weisungen ergänzt werden, gehen die institutionellen Maßnahmen, die die 
Verfahren bei der geschlechtergerechten Ausgestaltung der Arbeitsbedingungen, der Rekrutierung und der Aufstiegsförderung von Frauen begleiten, in ihrer Mehrzahl auf die allgemeine und spezifische Gleichstellungsgesetzgebung für die Bundesbehörden zurück. Das wichtigste Instrument ist hier das Amt der Gleichstellungsbeauftragten, die an den Personalverfahren beteiligt ist und die Berücksichtigung der substanziellen Ziele und die personalpolitischen Prozesse überwacht. Das zweite zentrale Instrument ist die Pflicht zur Erstellung von Gleichstellungsplänen für die jeweilige Organisation (d.h. Ministerien bzw. Ämter), und schließlich kann die Erstellung von Gleichstellungsberichten der Regierungen, an denen sich die Fortschritte der Gleichstellungsförderung der gesamten Bundesverwaltung ablesen lassen, gesetzlich vorgeschrieben sein.

\subsubsection{Gleichstellungsbeauftragte: Zu schwach institutionalisiert für ihre Umsetzungsaufgaben}

Das Amt der Gleichstellungs- oder Gleichbehandlungsbeauftragten (GBA) wurde in allen drei Bundesverwaltungen bereits in den 1980er Jahren vor der Verabschiedung der Bundesgleichstellungsgesetze bzw. Gleichstellungsweisungen eingerichtet, und ihre Rechte und Pflichten wurden durch die spezifische Gleichstellungsgesetzgebung weiter ausformuliert. In allen drei Bundesdiensten hat die GBA eine Doppelrolle: Sie ist Ansprechpartnerin für Beschäftigte in Diskriminierungsfragen und sie berät Vorgesetzte und Amtsleitung bei der Entwicklung und Umsetzung von Gleichstellungsmaßnahmen. Die genaue Rolle bei der Planung und Kommentierung der Personalpolitik ist jedoch nicht immer eindeutig definiert und akzeptiert, und die Ressourcen der GBA sind nur teilweise gesetzlich vorgegeben. In der Praxis variiert die Effektivität der Arbeit der GBA außerdem, weil ihre Tätigkeit die Aufgeschlossenheit des Arbeitsumfeldes für Gleichstellungsfragen, eine konstruktive Zusammenarbeit sowie ein hohes Maß an persönlicher Initiative und Kompetenz voraussetzt.

In Deutschland sind sowohl die Aufgaben und Kompetenzen der GBA als auch ihre Mitwirkungsrechte im spezifischen Gleichbehandlungsrecht ausführlich festgelegt. In Österreich sind grundlegende Regelungen der Gleichbehandlungsbeauftragten im Gleichbehandlungsgesetz festgelegt und ihre Aufgaben und Rechte in den ressortspezifischen Frauenförderplänen spezifiziert. In beiden Ländern sind sie in jedem Bundesministerium als zentrale Akteurin der Gleichstellungsproblematik sichtbar. Im schweizerischen Bundesdienst ist hingegen keine der deutschen und österreichischen Gleichstellungsbeauftragten vergleichbare konkrete und stabile Institutionalisierung der GBA gegeben: Hier schreiben weder das Gleichstellungsgesetz noch das Bundespersonalgesetz noch die Chancengleichheitsweisungen eine solche 
Funktion verbindlich vor. Vielmehr werden Gleichstellungsfragen in den verschiedenen Ressorts autonom behandelt und sind daher ,situativ institutionalisiert" - als eine zentrale Stabstelle von mehreren Personen oder eine einzelne Beschäftigte mit einem geringen Arbeitszeitanteil durch die Personalkommission (siehe Kapitel 4 und 7 zur Schweiz).

Tafel 8.1: Das Amt der Gleichstellungsbeauftragten im Drei-LänderVergleich

\begin{tabular}{|c|c|c|c|}
\hline & $\begin{array}{l}\text { Schweiz } \\
\text { (Ämter) }\end{array}$ & $\begin{array}{l}\text { Österreich } \\
\text { (Bundesmin.) }\end{array}$ & $\begin{array}{l}\text { Deutschland } \\
\text { (Bundesmin.) }\end{array}$ \\
\hline \multicolumn{4}{|l|}{ Verbindlichkeit } \\
\hline Bestellung & Ernennung & Bestellung & Wahl \\
\hline \multicolumn{4}{|l|}{ Mitwirkungsrechte } \\
\hline $\begin{array}{l}\text { Personalpolitische } \\
\text { Entscheidungen }\end{array}$ & - /o im EDA & + & ++ \\
\hline Strategische Planung & - /o im EDA & + & $(-)$ \\
\hline \multicolumn{4}{|l|}{ Reichweite } \\
\hline Zuständigkeitsbereich & $\begin{array}{l}\text { Geschlecht, Behinde- } \\
\text { rung, Mehrsprachig- } \\
\text { keit }\end{array}$ & $\begin{array}{l}\text { Geschlecht, ethnische } \\
\text { Zugehörigkeit, Religi- } \\
\text { on oder Weltanschau- } \\
\text { ung, Alter und sexuel- } \\
\text { le Orientierung }\end{array}$ & Nur Geschlecht \\
\hline $\begin{array}{l}\text { Hierarchische Zuord- } \\
\text { nung }\end{array}$ & Geschäftsleitung & Ressortleitung & $\begin{array}{l}\text { Leitung der Dienst- } \\
\text { stelle/der Personalab- } \\
\text { teilung }\end{array}$ \\
\hline Weisungsfreiheit & Weisungsgebunden & Weisungsfrei & Weisungsfrei \\
\hline $\begin{array}{l}\text { Budget } \\
\text { (Zeit/Personal) }\end{array}$ & $\begin{array}{l}\text { Geringe Teilzeit (im } \\
\text { EDA mehrere Stellen) }\end{array}$ & $\begin{array}{l}\text { Mehrere Teilzeit- } \\
\text { GBAs }\end{array}$ & $\begin{array}{l}\text { Nach Zahl der Mitar- } \\
\text { beitenden definiert }\end{array}$ \\
\hline $\begin{array}{l}\text { Ausdifferenziertheit } \\
\text { (Interne) Vernetzung }\end{array}$ & $\begin{array}{l}\text { Schwach: keine in der } \\
\text { BV, EPA }\end{array}$ & Stark (IMAG-BIG) & Mittelstark (IMA) \\
\hline $\begin{array}{l}\text { Anerkennung der } \\
\text { fachlichen Kompeten- } \\
\text { zen }\end{array}$ & Gering (hoch im EDA) & Gering & Informell \\
\hline $\begin{array}{l}\text { Voraussetzungen für } \\
\text { die Effektivität des } \\
\text { Amtes }\end{array}$ & $\begin{array}{l}\text { Schwach (mittelstark } \\
\text { im EDA) }\end{array}$ & Mittelstark & Stark \\
\hline
\end{tabular}

Quelle: eigene Darstellung auf Basis der Länderkapitel

Die deutschen GBAs sind nach wie vor allein für die Ungleichheit zwischen Frauen und Männern, für die Themen Vereinbarkeit von Beruf und Familie sowie sexuelle Belästigung zuständig und können ihre knappen Ressourcen auf diese Themenfelder konzentrieren, während die österreichischen GBAs auch für die Gleichbehandlung von Personen unterschiedlicher ethnischer 
Zugehörigkeit, Religion oder Weltanschauung, sexueller Orientierung und Alters zuständig sind. Im schweizerischen Fall wird Geschlechtergleichstellung gleichrangig mit Mehrsprachigkeit und Behindertenförderung unter Diversity Management subsumiert und damit in die Personalpolitik integriert.

Die GBA in der deutschen Bundesverwaltung hat durch ihre Wahl von den weiblichen Mitarbeiterinnen (auf vier Jahre) und ihre unmittelbare $\mathrm{Zu}$ ordnung zur Leitung der Dienststelle eine relativ starke Stellung und einen repräsentativen Charakter; sie kann daher in den Personalverfahren nicht leichthin übergangen werden. Die Beauftragten im schweizerischen und österreichischen Bundesdienst werden hingegen von der Ressortleitung bestellt - für fünf Jahre in Österreich und auf unbefristeter Zeit in der Schweiz. In Österreich sind die GBAs somit formal der Arbeitgeberseite zugeordnet, sie genießen aber in der Ausübung ihrer Aufgaben - genauso wie in Deutschland - eine gewisse Autonomie und können in ihren Anliegen weisungsfrei agieren. In der Schweiz besteht diese Autonomie hingegen nicht. Der starke Status der deutschen GBAs ergibt sich zudem aus der Tatsache, dass die Freistellung von der anderweitigen Arbeit entsprechend der Zahl der Beschäftigten gesetzlich auf die halbe (bis 600 Beschäftigte) bzw. die volle Stundenzahl (ab 600 Beschäftigte) im Bundesgleichstellungsgesetz festgelegt ist; in großen Bundesministerien können der GBA zudem MitarbeiterInnen zugeteilt werden. Die Ressourcenausstattung ist in den beiden anderen Ländern hingegen gänzlich ungeregelt: In Österreich ist die Anzahl der GBAs entsprechend der Größe des Ressorts festgelegt, jedoch ohne Vorgabe eines konkreten Zeitbudgets. Schweizerische GBAs sind mit Ausnahme des AuBendepartements mit einem geringen Zeitkontingent (10\% bis $20 \%)$ ausgestattet und werden je nach Amt von einer Arbeitsgruppe interessierter Beschäftigter unterstützt, wenn nicht die ganze Gleichstellungsarbeit gleich der Personalkommission übertragen wird.

Die Übernahme des Amtes kann Konsequenzen für den weiteren Berufsverlauf der Gleichstellungsbeauftragten haben. Zwar postulieren österreichische und deutsche Gesetze, dass den Gleichbehandlungsbeauftragten keine Nachteile aus ihrer Tätigkeit entstehen dürfen, doch kann die Ausübung des Amtes den weiteren Karriereverlauf beeinträchtigen, da die mit dem Amt verbundenen spezifischen Qualifikationen nicht zwangsläufig anerkannt werden (Interview in Deutschland und Österreich; vgl. Geppert/Lewalter 2012). Obwohl sich die GBAs für die Erfüllung ihrer Aufgaben Grundlagen des Personalrechts, Managementkompetenzen sowie Techniken der Konfliktberatung aneignen müssen, ist eine formale Anerkennung dieser Kompetenzen in keiner der drei Bundesverwaltungen geregelt. Im deutschen Gesetz ist immerhin eine „fiktive Nachzeichnung ihres beruflichen Werdegangs“ vorgesehen, die aber anhand einer Vergleichsgruppe nur eine Fortschreibung vergangener Beurteilungen (d.h. vor der Freistellung als GBA) nachzeichnet. Die Beratung bei der Umsetzung der Gleichstellungspolitik gegenüber Vor- 
gesetzten und Amtsleitung beruht auf den gesetzlich verankerten Mitwirkungsrechten der GBAs bei der Rekrutierung und Beförderung von Beschäftigten. In allen drei Bundesverwaltungen nehmen GBAs beratend an Besetzungsverfahren teil, in der Schweiz allerdings nur sehr begrenzt im Eidgenössischen Departement des Äußeren. Die GBAs der deutschen Bundesverwaltung genießen darüber hinaus ein breites und frühzeitiges gesetzlich verankertes Beteiligungsrecht an der Gestaltung aller gleichstellungsrelevanten Maßnahmen personeller, sozialer und organisatorischer Art. Ihre Beratungsrolle ist also wesentlich breiter gefächert als die der GBAs in der Schweiz und in Österreich, auch wenn sie in der Praxis nicht in allen Bereichen gleichermaßen umgesetzt wird: Bei Einstellungen sind die GBAs häufiger und nach klareren Regeln beteiligt als bei Beförderungen. In Österreich können GBAs jedoch gegebenenfalls die Klärung eines Diskriminierungsverdachts durch die Gleichbehandlungskommission veranlassen.

Die Tätigkeit der GBAs ist in der Regel auf das kurzfristige operative Geschäft konzentriert. An strategischer Personalplanung sind sie weniger beteiligt: Die GBAs des österreichischen Bundesdienstes sind zwar in einzelne strategische Personalentscheidungen eingebunden, ihre Mitgestaltungsmöglichkeiten sind jedoch von ihrer aktuellen beruflichen Position im Amt abhängig. Gleichstellungsbeauftragte des schweizerischen Bundesdienstes können lediglich im Rahmen der Konsultation zur mittelfristigen Personalstrategie des Bundesrates Stellung nehmen, welche das Eidgenössische Personalamt federführend betreut; mit Ausnahme des Außendepartements sind sie aber nicht an der Ausarbeitung und Konkretisierung von Plänen in den jeweiligen Ämtern beteiligt (siehe Kapitel 4 und 7 zur Schweiz). Auch in Deutschland ist eine Beteiligung an der mittelfristigen Personalplanung nicht garantiert und musste mehrmals vor Verwaltungsgerichten eingeklagt werden. Beispielhaft ist der Fall einer GBA im Bundesfinanzministerium, die bis vor das Bundesverwaltungsgericht ging, das schließlich das Recht auf Teilnahme der GBA an personalpolitisch relevanten Führungsklausuren des Amtes bestätigte (siehe Kapitel 5 zu Deutschland).

In Österreich wirkt die Bundesgleichbehandlungskommission, die einen Diskriminierungsverdacht und Konfliktfälle im Bundesdienst unabhängig untersucht und Gutachten erstellt, gewissermaßen als „Rute im Fenster“; sie kann im Konfliktfall von den GBAs angerufen werden. Solche starken rechtlichen Gelegenheitsstrukturen haben eine höhere Mobilisierung des Rechts zur Folge: Allein zwischen 2005 und 2012 wurden 125 Fälle verhandelt. Im Gegensatz dazu gab es bei der schweizerischen Schlichtungskommission, die nur auf Antrag tätig wird, seit Bestehen lediglich fünf Fälle (siehe auch Fuchs et al. 2009).

Eine weitere wichtige Ressource ist schließlich die Vernetzung der GBAs der unterschiedlichen Ämter und Ministerien der Bundesverwaltung. Die ressortübergreifende Koordination ist nicht nur für den Informations- und 
Erfahrungsaustausch über die jeweilige Umsetzungspraxis der betrieblichen Gleichstellung wichtig, sondern sie trägt auch zur Professionalisierung der Arbeit der GBAs bei, weil sich ein gemeinsames Berufsverständnis herausbilden kann. Dabei werden außerdem die spezifischen Kompetenzen der GBAs (z.B. Kenntnisse des Personalrechts, Konfliktlösungskompetenzen) als Expertinnenwissen sichtbar und in einem fachlichen Kontext anerkannt.

In der Bundesverwaltung Österreichs ist die Vernetzung der GBAs offiziell organisiert und breit gefächert: Die Interministerielle Arbeitsgruppe für Gleichbehandlungsfragen, die zweimal im Jahr tagt, versammelt die Vorsitzenden der ministeriellen Arbeitsgruppen, aber auch GBAs vieler bundesnaher Organisationen. In der deutschen Bundesverwaltung war die Koordinierung der GBAs bisher weniger institutionalisiert: Die ressortübergreifende Zusammenarbeit der GBAs der obersten Bundesbehörden ist zwar durch die gemeinsame Geschäftsordnung der Bundesministerien festgelegt, allerdings fehlte der 1991 gegründeten Interministeriellen Arbeitsgruppe der Gleichstellungsbeauftragten (IMA) bis 2015 eine klare gesetzliche Grundlage für ihre Arbeit. Im schweizerischen Bundesdienst wurde diese Koordinierung im Rahmen des sog. Forums, einer interdepartementalen Arbeitsgruppe für alle Gleichstellungsbeauftragten, 2012 nach dem Versuch einer stärkeren Institutionalisierung aufgelöst. Bestrebungen zur Wiederaufnahme scheinen sich zurzeit aber auf Weiterbildungsveranstaltungen zu beschränken.

$* * *$

Die Verbindlichkeit des Instruments der GBA ist in der deutschen Bundesverwaltung am größten, wo sie durch die direkte Wahl eine hohe Legitimität hat und durch ihre quasi-systematische Zuordnung zur Personalabteilung der Bundesministerien einen direkten und zudem rechtlich garantierten Zugang zu den Personalverfahren. Dass die GBAs notfalls ihre Rechte einklagen können (und dies auch zuweilen tun), ist Ausdruck der - im Vergleich zur Schweiz oder zu Österreich - hohen Verbindlichkeit dieses Amtes. Zudem sind die Mitwirkungsrechte der GBAs in Deutschland weitgehend ausdifferenziert (bis hin zur Personalplanung in den Ministerien) und festgeschrieben; dass sie allein auf die Gleichstellung begrenzt sind, ermöglicht eine Fokussierung der Ressourcen und eine Spezialisierung auf Gleichstellungsfragen. Die schweizerische Alternative der vollständigen Integration von Gleichstellungsfragen in die Personalpolitik bringt eine Politik hervor, deren Effektivität ohne ein proaktives Gegengewicht starker GBAs exklusiv von der Leitungsebene abhängt. Sowohl in der Schweiz als auch in Deutschland ist die Reichweite dieses Amtes weitgehend auf das jeweilige Ministerium begrenzt, da ein politischer und ressortübergreifender Austausch nur begrenzt funktioniert und keine größeren Aktivitäten ermöglicht. Dies ist in Österreich tendenziell anders, wo sich die Interministerielle Arbeitsgruppe für Gleich- 
stellungsfragen auch mit darüber hinausreichenden gleichstellungspolitischen Themenstellungen befassen kann und ein Austausch gewährleistet ist.

\subsubsection{Gleichstellungspläne der Bundesministerien und Ämter: Strategisches Instrument für geschlechtergerechte Personalplanung}

Die Gleichstellungspläne, zu deren Erstellung die Bundesministerien und Ämter gesetzlich verpflichtet sind, haben eine Doppelfunktion: Sie sind ein Planungs- und gleichzeitig ein Kontrollinstrument und zielen auf die Veränderung der Personalpolitik in ihrer Substanz. Vor allem konkretisieren sie das allgemeine Gleichstellungsziel in Teilzielen und schreiben Instrumente zu deren Umsetzung fest. Im Rahmen eines Monitoring wird dann die Zielerreichung für jedes einzelne Ministerium oder Amt in bestimmten Zeitabständen überprüft, indem die Gleichstellungspläne z.B. Indikatoren über die Verteilung von Frauen und Männern auf die Status- oder Lohngruppen erfassen und Maßnahmen für die Zielerreichung festgelegen.

Verantwortlich für die Erstellung der Gleichstellungspläne ist in allen drei Ländern die Personalverwaltung, doch ist die GBA an der Erstellung des Gleichstellungplans unterschiedlich beteiligt. Prinzipiell gilt die Beteiligung der Gleichstellungsbeauftragten als eine Garantie für die Angemessenheit von Analyse und Zielformulierung, aber auch als kontrollierender Mechanismus dafür, dass überhaupt ein Plan erstellt wird.

So nehmen die österreichischen Gleichbehandlungsbeauftragten sogar eine führende Rolle bei der Erstellung und Kontrolle des Frauenförderplans in ihrem Ressort ein: Die Frauenförderpläne werden hier alle sechs Jahre von den Arbeitsgruppen für Gleichbehandlungsfragen (die sich aus allen GBAs im Ressort zusammensetzen) erarbeitet und von der Ressortleitung erlassen und alle zwei Jahre angepasst. Allerdings sind die Inhalte der Frauenförderpläne nicht festgelegt, vielmehr können sie zwischen den Ressorts variieren, sodass die Bundesministerien unterschiedliche Schwerpunkte setzen und somit die Vereinbarkeit oder die Karriereförderung in den Blick nehmen können (siehe Kapitel 6 zu Österreich). Sie enthalten immer auch quantitative Zielgrößen zu den Frauenanteilen nach bestimmten Beschäftigten- und Lohngruppen, die als Bewertungskriterium für die im Gleichbehandlungsbericht des Bundes abgebildeten Indikatoren dienen. Beide Instrumente, die Frauenförderpläne und der Gleichbehandlungsbericht des Bundes, sind öffentlich zugängliche Dokumente. Die regelmäßige Publikationspflicht erhöht zwar die Verbindlichkeit für die Planung von Gleichstellungsmaßnahmen, regt aber kaum interne Reflexionsprozesse zur Verbesserung von Maßnahmen oder zur Abstimmung von Maßnahmen zwischen Ministerien an, sondern dient eher der Legitimierung von Gleichstellungsagenden. 
In der schweizerischen Bundesverwaltung obliegt die Entwicklung der Personalstrategie dem zentralen Personalamt, das in diesem Zuge auch ein eigenes Kapitel zur Geschlechtergleichheit verfasst. Zur Kontrolle werden jährlich Berichte zur Chancengleichheit der Bundesverwaltung veröffentlicht, in denen Zielbänder für die Anteile von Frauen in bestimmen Status- und Lohngruppen festgelegt und der jeweils aktuelle Stand berichtet werden. Für die Maßnahmen innerhalb der einzelnen Departemente und Ämter werden die sogenannten Fortschrittsberichte verfasst, die auf Basis der berichteten Kennzahlen die internen Maßnahmen zur Förderung der Gleichstellung festlegen; diese internen Berichte werden jedoch nicht öffentlich gemacht. Die Gleichstellungbeauftragten erstellen zwar diese jährlichen Fortschrittsberichte ihres eigenen Amtes, sind aber am jährlichen Gesamtbericht der Bundesverwaltung nur beratend beteiligt.

In Deutschland ist die Erstellung von Gleichstellungsplänen für jedes Ressort im Bundesgleichstellungsgesetz vorgeschrieben: Die Pläne sollen von der für die Personalpolitik verantwortlichen Zentralabteilung in den Bundesministerien erstellt und der GBA vorgelegt werden. Die GBA einer Dienstelle ergänzt, kommentiert und bewilligt abschließend den Gleichstellungsplan im Rahmen ihrer Mitwirkungsrechte. Sie kann gegen den Plan gegenüber ihren Vorgesetzten (in der Regel die Leitung der für das Personal zuständigen Zentralabteilung der Bundesministerien) Einspruch einlegen, Gegenvorschläge machen und im schlimmsten Fall gerichtlich gegen einen unzureichenden Plan vorgehen (siehe Kapitel 5 zu Deutschland). In Deutschland müssen die Gleichstellungspläne den aktuellen Stand anhand quantifizierter Ziele sowie Maßnahmen und Fristen für deren Umsetzung enthalten. Zwischenberichte sollen im Zweijahrestakt die Fortschritte illustrieren. Die Umsetzung der Pläne wird jedoch nicht zentral kontrolliert, und es besteht dadurch eine erwiesene große Bandbreite von unterschiedlichen Praxen unter den Ressorts: Laut dem zweiten Erfahrungsbericht fehlten etwa im Jahr 2009 bei der Hälfte der Ressorts die Gleichstellungspläne. Ein weiterer Teil genügte lediglich der formalen Pflicht, ein dritter Teil nutzte den Bericht tatsächlich als Instrument des gleichstellungspolitischen Monitorings in der Personalpolitik. Die Wirkungskraft des Instruments der Gleichstellungspläne ist ohne die Bündelung der Befunde zu übergreifenden Berichten sowie eine ressortübergreifende Abstimmung jedoch gering. Daher stellt die Berichterstattung gewissermaßen den „Schlussstein“ der institutionellen Maßnahmen der Gleichstellungspolitik des Bundesdienstes dar.

$* * *$

Der Vergleich zeigt, dass die Inhalte der Frauenförderpläne kaum standardisiert sind und unterschiedliche Schwerpunkte setzen. Zudem wird in keiner der Bundesverwaltungen die konkrete Zielerreichung verbindlich abgeprüft. Das Risiko eines Ausweichverhaltens ist daher hoch. Eine gewisse Verbind- 
lichkeit haben die Frauenförderpläne in Österreich und in der Schweiz durch die Veröffentlichungspflicht, die in Deutschland hingegen fehlt. Hier ist es von der GBA abhängig, inwiefern es ihr gelingt, auf die Erstellung des Plans $\mathrm{zu}$ dringen und seine Inhalte zu beeinflussen.

Eine gewisse Wirksamkeit entsteht zudem in der Schweiz, wo die Gleichstellungspläne in die allgemeine Personalpolitik integriert sind und somit die regelmäßige und verbindliche Umsetzung gewährleistet ist. Allerdings wirkt die GBA kaum an deren Erstellung mit, und die personalpolitischen Evaluationsberichte bieten wenig qualitative Informationen zur Gleichstellung, ganz im Gegensatz zu den amtsinternen Fortschrittsberichten. In Deutschland könnten die internen Gleichstellungspläne grundsätzlich als wirksames Planungs- und Controllinginstrument eingesetzt werden. Aber es besteht doch wenig Verbindlichkeit für deren Erstellung, was die Umsetzung - wie auch in den anderen Ländern - abhängig macht von den Mitwirkungsrechten und dem Engagement der Gleichbehandlungsbeauftragten und deren Durchsetzungskraft sowie dem gleichstellungspolitischen Engagement der Personalabteilung. Günstig ist außerdem, wenn die Ergebnisse für jede Behörde Teil eines ressortübergreifenden Berichts werden, der Stand und Entwicklung und somit den Erfolg der spezifischen Gleichstellungsvorschriften öffentlich sichtbar und vergleichbar macht.

\subsubsection{Berichterstattung zur Erhöhung der Verbindlichkeit von autonomem, aber zielgebundenen Handeln in der Bundesverwaltung}

Die Gleichstellungspolitik für den Bundesdienst wird allen drei Ländern durch ein Ressort koordiniert, das auch für die regelmäßige Berichterstattung über die gleichstellungspolitischen Aktivitäten der Gesamtverwaltung verantwortlich ist. In Deutschland hat das Bundesministerium für Familie, Senioren, Frauen und Jugend (BMFSFJ) - genauer gesagt: seine Gleichstellungsabteilung - diese Koordinierungsaufgabe inne. Diese Abteilung steht zudem den GBAs als Klärungsinstanz zur Verfügung und agiert als Wissensvermittler gegenüber den obersten Bundesbehörden und dem Parlament. In Österreich leitet die Bundesfrauenministerin die „Interministerielle Arbeitsgruppe für Gleichbehandlungsfragen“, die die GBAs aller Ressorts versammelt, die ja für die Erstellung und Evaluierung der Gleichstellungspläne ihrer Ämter verantwortlich sind - hier scheint der Informationsaustausch zumindest punktuell funktional organisiert. Im Fall der Schweiz übernimmt das Eidgenössische Personalamt die Planung und Umsetzung der Personalpolitik der Bundesverwaltung, in der gleichstellungspolitische Vorhaben integriert sind. Somit koordiniert es auch die Gleichstellungsberichterstattung. Allerdings 
verfügt das EPA in der Gesamtverwaltung nur über ein relativ geringes politisches Gewicht.

Die Berichterstattung selbst dient im Idealfall der Abstimmung der Gleichstellungspolitik zwischen den Ministerien und macht die Erfolge und Misserfolge der unterschiedlichen Strategien sichtbar und vergleichbar. Eine regelmäßige Gleichstellungsberichterstattung findet man in allen drei Bundesverwaltungen: In der Schweiz formuliert das Eidgenössischen Personalamt einerseits gleichstellungspolitische Zielsetzungen in einem eigenen Kapitel der jährlichen Berichte zur Personalstrategie, andererseits verfasst es aber auch jährlich eigene spezielle Berichte zur Chancengleichheit. In Deutschland legt das Bundesgleichstellungsgesetz fest, dass die Bundesregierung alle vier Jahre einen sog. „Erfahrungsbericht“ für die gesamte Bundesverwaltung vorlegen muss, der zuletzt mithilfe eines universitären Forschungsinstituts erstellt wurde. In Österreich wird der eigene Gleichbehandlungsbericht des Bundes auf Basis der Frauenförderpläne alle zwei Jahre vom Frauenministerium erstellt und präsentiert den erreichten Stand in den einzelnen Ressorts mittels einheitlicher Indikatoren.

Die in den Berichten präsentierten Zahlen dienen dem Vergleich zwischen Ist- und Soll-Zustand. In Österreich hat die Definition von Frauenquoten und in der Schweiz die quantifizierten "Zielbänder" somit eine wichtige Orientierungsfunktion. Hingegen sind in der deutschen Bundesverwaltung Geschlechterquoten trotz ihrer starken gesetzlichen Verankerung kaum relevant für die Evaluierung der gleichstellungsgesetzlichen Vorhaben, weil die Evaluierung des Bundesgleichstellungsgesetzes nur „ressortübergreifend“ aggregierte Daten und weniger ressortvergleichende Daten präsentiert.

Die Methodik des Benchmarkings der evaluierten Ressorts ist somit sehr unterschiedlich: In Österreich enthalten die Gleichbehandlungsberichte zumindest für die Beschäftigungspartizipation von Frauen vergleichbare Indikatoren für alle Ressorts. Obwohl die allgemein zugängliche Berichterstattung der einzelnen Bundesministerien es erlauben würde, werden die Unterschiede zwischen den Ministerien nicht thematisiert. Durch die Darstellung sind die umgesetzten Maßnahmen mit den dabei getroffenen Schwerpunktsetzungen, spezifischen Frauenförderungen und Verletzungen des Gleichbehandlungsgebots hingegen kaum vergleichbar bzw. weisen wenig Aussagekraft auf. Die deutschen Berichte (von denen schon fünf erschienen sind) sind nicht standardisiert: Der direkte und klare Vergleich der Ressorts ist hier nicht das Ziel. Vielmehr werden sie als allgemeine „Erfahrungsberichte" formuliert, der die gleichstellungsrelevanten Merkmale für alle Bundesministerien zusammenfasst. Ein Benchmarking im engeren Sinne wird in der Schweiz nicht von der Bundesverwaltung, wohl aber in inoffizieller Manier von der Gewerkschaft für den öffentlichen Dienst (VPOD) durchgeführt; diese veröffentlicht jährlich die nach Departementen aufgeschlüsselten Daten zu Frauenanteil, Teilzeitarbeit im Kader, Leistungsbeurteilungen und Prämien. Die öffentliche 
Sichtbarkeit der gleichstellungspolitischen Aktivitäten im Bundesdienst hängt letztlich auch von der medialen Aufmerksamkeit für das Thema ab. In Deutschland lenkte die Debatte zur Einführung einer gesetzlichen Frauenquote für die Aufsichtsräte börsennotierter Unternehmen kurzfristig die Aufmerksamkeit auf die Führungsetagen der Bundesministerien, um erfolgreiche Beispiele der Frauenförderung (Bundesfamilienministerium) zu identifizieren.

$* * *$

Obwohl in allen drei untersuchten Bundesverwaltungen technisch möglich, werden die Vergleiche zwischen den Ressorts kaum zur Durchsetzung des Gleichstellungsziels genutzt. Hierfür ist die Autonomie der Bundesministerien und Ämter in Personalfragen ursächlich, die als ehernes Prinzip der Behörden akzeptiert ist (Interviews Deutschland).

In der Schweiz findet sich eine gute Strategie des öffentlichen Monitorings - ein öffentlich zugänglicher Bericht, der durch das EPA erstellt wird, und das inoffizielle Benchmarking durch die Gewerkschaft. Der Gleichstellungsbericht für die österreichische Bundesverwaltung wäre dahingehend weiterzuentwickeln, dass der einfache Vergleich der Indikatoren durch vergleichende Analysen im Hinblick auf die spezifischen Bedingungen in den Ministerien unter Einbeziehung der Bewertung ergriffener Maßnahmen ergänzt wird. Für die deutsche Bundesverwaltung fehlt gänzlich eine zielgerichtete, standardisierte und analytische Berichterstattung, die auch die vergleichende Bewertung von gleichstellungspolitischen Erfolgen erlaubt. Die für 2015 vorgesehene Novellierung des Bundesgleichstellungsgesetzes sieht aber die Einführung eines Gleichstellungsindex vor, der die Leistungen der obersten Bundesbehörden vergleichbar machen würde.

\subsection{Fazit: Licht und Schatten in der Gleichstellungspolitik der drei Bundesverwaltungen}

Die vergleichende Analyse zeigt, dass das Personalrecht in allen drei Ländern die Basis darstellt und durch allgemeine arbeitsrechtliche Vorschriften ergänzt wird (Teilzeitarbeit, Elternfreistellungen). In den 1990er Jahren (zuerst in der Schweiz und in Österreich, zuletzt auch in Deutschland) entwickelten sich dann spezielle Rechtsvorschriften, die spezifisch die Gleichstellung im Bundesdienst regeln: In allen drei Ländern betonen sie die Notwendigkeit der Förderung der Vereinbarkeit und die Aufstiegsförderung von Frauen. In Österreich und Deutschland institutionalisieren sie zudem Verfahrensregeln, welche die für die Personalpolitik zuständigen Akteure ergänzen. In der Schweiz werden die personalrechtlichen Verfahren von Gleichstellungsvor- 
schriften weniger berührt; die Kontrolle des Gleichstellungsgebots wird weitgehend auf die individuelle Überprüfung der Ämter verlagert. Im Rahmen des Personalcontrollings werden jedoch Verfahren zur Überprüfung der Gleichstellungsfortschritte institutionalisiert. Ohne Zweifel sind die gesetzlichen bzw. untergesetzlichen Regelungen zur Gleichstellungspolitik des Bundesdienstes vorbildlich für die Privatwirtschaft, da die Regelungen hohe formale Standards für die Vereinbarkeit von Beruf und Familie, die Rekrutierung von Frauen und für die Karriereförderung von Frauen etablieren und die Personalverfahren in den Behörden des Bundesdienstes zusätzlichen institutionalisierten Verfahren (Mitwirkung von Gleichstellungsbeauftragten, Erstellung von Förderplänen und Berichten) unterworfen sind. Hiermit signalisieren die obersten Bundesbehörden, dass sie sowohl attraktive als auch um Mitarbeiterinnen bemühte Arbeitgeber sein wollen.

Diese Strategie, die allgemeine modernisierte personalrechtliche Vorschriften mit spezifischen Frauenfördergesetzen und -weisungen verbindet, ist weitgehend kohärent und unterstützt den Anstieg des Frauenanteils an den Beschäftigten im Bundesdienst - auch in den Führungsetagen.

Betrachtet man den Mix der substanziellen Maßnahmen der Gleichstellungspolitik, erweist sich das Policy-Regime als durch normativ kohärent mit dem „modifizierten Ernährermodell“: Frauen wird vor allem durch Teilzeitarbeit und Vereinbarkeitsmaßnahmen eine kontinuierliche Beschäftigung in den Bundesdiensten ermöglicht. Eine konsistente Implementation von verpflichtender - und effektiver - Aufstiegsförderung ist hingegen nicht zu beobachten. Der langfristige Verbleib von Frauen in Teilzeitkarrieren und die langsamen Fortschritte beim Aufstieg in das mittlere und gehobene Management entsprechen somit durchaus den normativen Erwartungen nach diesem Modell, in dem Frauen gewissermaßen als Flexibilitätsreserve bereitstehen, aber noch nicht zu einer gleichen Teilhabe an allen Statuspositionen gefördert werden. Eine umfassende Gleichstellung ist damit nicht gewährleistet.

Zwar sind Maßnahmen zur Laufbahnförderung für Frauen (Quotierung bei Einstellungsverfahren oder Aufstieg) vorhanden, werden aber nicht immer stringent umgesetzt bzw. führen zu Konflikten in der Praxis der GBAs. In allen drei Ländern erweisen sich dabei die Definition des Qualifikationsbegriffs (zu wenig ausdifferenziert) und das Fehlen einer systematischen Anerkennung von Schlüsselqualifikationen als Nadelöhr für eine diskriminierungsfreie vergleichende Bewertung von Qualifikationsprofilen konkurrierender BewerberInnen. Um eine diskriminierungsfreie Anwendung zu erleichtern, wäre eine Weiterentwicklung der Bewertungskriterien unter Einbeziehung von GBA, Vertreterinnen der WissenschaftlerInnen und der zuständigen Fachabteilung ein hilfreicher Schritt.

Das gezielte Angebot von Weiterbildungsmaßnahmen und Coachings oder Mentoring-Programmen für angehende Führungskräfte trägt dem Problem, dass zu wenige kompetente Frauen in die Verfahren gelangen, in vielen 
Häusern bereits Rechnung. Von der fortbestehenden Teilzeitstrategie und den damit verbundenen Problemen einmal abgesehen, erscheinen die einzelnen Maßnahmen zur Karriereförderung gut miteinander koordiniert. Allerdings sind die einzelnen Bundesministerien und -ämter nicht verpflichtet, diese Angebote anzubieten, und es besteht auch keine systematische und vergleichende Auswertung, sodass die aktive Karriereförderung bislang ein wenig verbindliches Instrument ist.

Die Verzahnung von substanziellen mit institutionellen Maßnahmen erweist sich als eine unerlässliche Bedingung für eine gute Umsetzung des Gleichstellungsauftrags. Die starke Autonomie der Verwaltungseinheiten sorgt jedoch wiederum für große Varianzen zwischen den Ressorts, vor allem in der Schweiz, wo das Gleichstellungsgesetz für öffentliche und private Arbeitsverhältnisse gleichermaßen gilt und dementsprechend große Spielräume für die Umsetzung belässt. Die Ausgestaltung der Gleichstellungspolitik ist damit anfällig für „Hauspolitiken“ und persönliche Einflüsse. In den anderen beiden Ländern sind die Vorschriften für die Bundesdienste zwar angepasst, aber auch hier ist die Verbindlichkeit gering und die Kontrolle der Umsetzung nicht gewährleistet, sodass sich auch hier eine große Varianz bei der Umsetzung herausbildet. Eine stärkere Standardisierung, wie sie im Ansatz auch im deutschen Gleichstellungsgesetz für den Bundesdienst festgeschrieben ist (z.B. die Ausstattung und die Rechte der GBA), sowie eine verbesserte personalpolitische Anerkennung der Kompetenzen und Tätigkeiten der Gleichstellungsbeauftragten würden die Mitwirkungs- und Kontrollfunktion erheblich stärken und im Ergebnis zu einer größeren Konsistenz der Gleichstellungstrategien in allen Ressorts führen.

Wenngleich die institutionellen Maßnahmen zur Umsetzung der gleichstellungspolitischen Vorhaben für den Bundesdienst in den drei Ländern relativ ausdifferenziert sind - im Zeitverlauf wurden sie präzisiert und immer enger mit der allgemeinen Personalpolitik koordiniert -, bietet keines der drei Länder ein effektives und rundum konsistentes Monitoringsystem. Dieses müsste auf einer regelmäßigen und weitreichenden Mitwirkung der GBAs basieren, wie sie im Prinzip in der deutschen Verwaltung vorhanden ist. Dynamische Mess- und Berichtsmethoden nach österreichischem Vorbild scheinen geeignet, die Basis für ein standardisiertes Benchmarking zu legen. Die Systematisierung des Vergleichs der einzelnen Ressorts auf Basis einer qualitativen und quantitativen Bewertung, die es nur im Ansatz in Österreich und der Schweiz gibt, erlauben fundierte Schlüsse über die Effektivität der Maßnahmen. Fortschrittsberichte etwa, wie es sie in der Schweizer Bundesverwaltung bereits gibt, sind schließlich ein hilfreiches Instrument, die Lehren aus den Bestandsaufnahmen regelmäßig zu überprüfen. Grundsätzlich sind in allen drei Ländern die Beschwerde- und Einspruchsmöglichkeiten der GBAs zu begrenzt, wenn ihre Rechte entgegen den Regeln nicht berücksichtigt werden. Und schließlich ist auch zu bedenken, dass die professionellen Kom- 
petenzen der Frauen, die das Amt der GBA ausfüllen, oftmals weder angemessen als Beitrag zur Organisationsentwicklung wertgeschätzt noch in nachfolgenden Berufsverläufen berücksichtigt werden.

Ein systematisches Problem der Gleichstellungspolitik in der Bundesverwaltung zeigt sich im Ländervergleich in allen drei Fällen. Zwar erweist sich formal die institutionelle Gleichstellungspolitik als gut verzahnt mit der allgemeinen Personalpolitik der Ämter, insbesondere in der Schweiz, wo die Gleichstellung ein Planungselement der Personalpolitik des EDA ist. Allerdings sind hier Normen und Logiken der Gleichstellungsstrategie der Personalpolitik untergeordnet, ohne dass diese eine Veränderung im Kern ihrer Grundprinzipien erfahren würde. Wie die Untersuchung zu Vorstellungen über Gleichstellung in der schweizerischen Bundesverwaltung gezeigt hat, reflektieren Beschäftigte und Vorgesetzte durchaus männliche tradierte und konnotierte Beschäftigungs- und Verfügbarkeitsnormen. Gleichstellung wird im Verständnis dabei tendenziell auf Vereinbarkeit und Frauen in Führungspositionen reduziert, während Organisationsstrukturen ausgeklammert bleiben. Gleichzeitig wird ein „,meritokratischer Individualismus“ gestärkt: Wenn Frauen den gleichen Normen wie Männer entsprechen und sich an strukturelle Rahmenbedingungen anpassen, haben sie auch die gleichen Aufstiegschancen.

Inkonsistenzen ergeben sich dort, wo bestehende Strukturen vermeintlich geschlechterneutraler Regelungen eben nicht einer systematischen Revision unterzogen und die gleichstellungspolitischen Aspekte als systemfremd empfunden werden. Hierzu gehören die Anwendung von Quotenregelungen, die Verfahren und Kriterien des Leistungsbeurteilungswesens und schließlich die Verfahren zu Beförderungsentscheidungen. Eben gerade die Definition von Qualifikationen und Leistungen sowie die Bewertung von Phasen der Teilzeitarbeit oder die Anerkennung von Schlüsselqualifikationen sind Probleme, die seit Langem in der personalwissenschaftlichen Debatte thematisiert werden. Solange die Verwaltungsmodernisierung personalpolitische Mechanismen ausspart und das Leitbild nicht entsprechend angepasst wird, wird eine geschlechtersensible Anwendung der gleichstellungspolitischen Anforderungen immer kontextabhängig bleiben.

Außerdem erscheint das notwendige Gleichstellungsmonitoring und -controlling tendenziell als schwer vereinbar mit der formalen und traditionell gewachsenen Autonomie der Ämter bei der Umsetzung bundesgesetzlicher Vorhaben. Dabei erweisen sich ausdifferenzierte Monitoringinstrumente, die verfeinerte Indikatoren, kontextgebundene Interpretationen und konkretere Ziele sichtbar und für den politischen Prozess handhabbar machen, als zentrale Instrumente für eine gute Gleichstellungspraxis. Eine politisch gestärkte Koordinierung der gleichheitspolitischen Aktivitäten auf der Ebene der Gesamtbundesverwaltung wäre hier sinnvoll, um die Effektivität des Gleichstellungsgesetzes zu erhöhen und die Wirkungen der Gleichstellungspolitik auch 
deutlich in der Öffentlichkeit sichtbar zu machen. Solange nicht konkrete Ergebnisse den verantwortlichen Einheiten zuzuordnen sind, diese zwischen den Einheiten verglichen werden können und solange auf eine Gesamtbewertung durch eine zentrale Instanz verzichtet wird, bleibt das Monitoringsystem hinter seinem Potenzial als Kontrollinstrument weit zurück. Eine öffentliche Diskussion von Gleichstellungszielen und -erfolgen würde auch dazu beitragen, in den Behörden und Ämtern einen organisationskulturellen Wandel insgesamt anzustoßen.

Gewiss hat der Bundesdienst in allen drei Ländern mit den ausdifferenzierten Regelungen zur Vereinbarkeit von Beruf und Familie, den institutionellen Rahmenbedingungen für die Begleitung personalpolitischer Prozesse und der nach wie vor relativ hohen Beschäftigungssicherheit eine Vorbildfunktion für private Arbeitgeber. Aber auch hier spiegeln sich die gesellschaftlichen Strukturen geschlechterspezifischer Ungleichheit wider - bei der Bezahlung und beim Zugang zu Führungspositionen. Allerdings ist es nicht den Akteuren im öffentlichen Dienst anzulasten, dass die Entwicklung in Richtung einer verbesserten Gleichstellung von Frauen und Männern nicht zügiger verläuft, da die gesetzlichen Rahmenbedingungen weitgehend angewandt werden. Insofern zeigt die vergleichende Analyse Ansatzpunkte für eine Überarbeitung gleichstellungspolitischer Gesetze - sowohl im Bereich substanzieller als auch institutioneller Regelungen. Allerdings ist ein tiefgreifender Wandel nicht allein durch gesetzliche Reformprojekte zu erreichen, wenn diese nicht aus einem übergreifenden gesellschaftspolitischen Prozess resultieren, in dem die öffentliche Verwaltung selbst an der Definition ihres Selbstverständnisses als moderne Personalverantwortliche und gesellschaftliche Akteure mitwirkt.

Mit der Einbettung der Gleichstellungspolitik der Bundesverwaltung in das jeweilige Geschlechterregime wird aber auch deutlich, dass nicht Instrumente allein über Effizienz oder Ineffizienz von Gleichstellungspolitik entscheiden, sondern dass sie sich erst im Kontext mit Umsetzungsregeln entfalten. Selbst wenn sich solche Kontexte stark ähneln wie in unserer Analyse, können sich die Folgen wesentlich unterscheiden. Dementsprechend gibt es einige Schlussfolgerungen aus den Erkenntnissen der vorliegenden Analyse.

Implementationsanalysen sind hoch relevant und beleuchten auch die ,gelebte Praxis“. Solche vergleichenden Analysen und Evaluationen sollten die Literatur zu Policy Design und die Instrumentenwahl mit der Prüfung jener Hypothesen verknüpfen, die aus der bisherigen ,feminist comparative policy"- Forschung abgeleitet werden können (vgl. Mazur o. J.: 13-16), etwa zur politischen Gelegenheitsstruktur, zu den Akteurskoalitionen oder dem Geschlechterregime der jeweiligen Länder. Für eine umfassende Analyse sollten hier auch organisationssoziologische Fragen rund um die ,vergeschlechtlichte Organisation“ integriert werden. 
Für anwendungsorientierte Forschungsfragen und Evaluationen zur betrieblichen Gleichstellung öffentlicher Arbeitgeber gilt es, klare Indikatoren zu entwickeln, welche die Umsetzung der Instrumente in die Praxis überprüfbar und quantifizierbar machen. Das gilt etwa für die hier qualitativ belegte Hypothese, dass stark verbindliche institutionelle Grundlagen ein größeres Ausma $ß$ und effektivere substanzielle Maßnahmen hervorbringen. Auch eine Messung der tatsächlichen Nutzung der Handlungsspielräume von GBAs ist wünschenswert. Die Indikatoren sollten mit Gewinn qualitative und quantitative Aspekte umfassen. So braucht es auf personalwirtschaftlicher Ebene mehr vergleichbare Daten im Zeitverlauf, etwa zu Erwerbsunterbrechungen, um Erfolge und Probleme in der Vereinbarkeitspolitik genauer zu evaluieren.

Implementationsanalysen und die Entwicklung valider Indikatoren würden in einem weiteren Schritt die Messung von Wirkungen und Vorteilen einer guten Gleichstellungspolitik ermöglichen. 


\section{Literatur}

Acker, Joan (1990): Hierarchies, Jobs, Bodies: A Theory of Gendered Organizations. In: Gender and Society, Jg. 4, H. 2, S. 139-158.

Acker, Joan (2012): Gendered organizations and intersectionality: problems and possibilities. In: Equality, Diversity and Inclusion: An International Journal, Jg. 31, H. 3, S. 214-224. Online verfügbar unter DOI 10.1108/02610151211209072.

Andresen, Sünne; Dölling, Irene (2005): Umbau des Geschlechter-Wissens von ReformakteurInnen durch Gender Mainstreaming. In: Behning, Ute; Sauer, Birgit (Hg.): Was bewirkt Gender Mainstreaming? Evaluierung durch Policy-Analysen. Frankfurt/M.: Campus, S. 171-187.

Andresen, Sünne; Dölling, Irene; Kimmerle, Christoph (2003): Verwaltungsmodernisierung als soziale Praxis. Geschlechter-Wissen und Organisationsverständnis von Reformakteuren (Interdisziplinäre Organisations- und Verwaltungsforschung 12). Opladen: Leske + Budrich.

Appelt, Erna (Hg.) (2009): Gleichstellungspolitik in Österreich. Eine kritische Bilanz. Innsbruck: Studienverlag.

Auer, Manfred; Welte, Heike (2009): Betriebliche Gleichstellungspolitik - top oder flop? In: Appelt, Erna (Hg.): Gleichstellung in Österreich - eine kritische Bilanz. Innsbruck: Studienverlag, S. 181-198.

Auer, Peter; Bauer, Christian; Kreisky, Eva (1977): Frauen im öffentlichen Dienst Öffentliche Dienste für Frauen. Endbericht im Auftrag des Magistrats der Stadt Wien. Wien.

Autorinnenkollektiv (2009): Gender Budgeting in Österreich - Möglichkeiten und Grenzen einer politischen Gleichstellungsstrategie. In: Appelt, Erna (Hg.): Gleichstellungspolitik in Österreich. Eine kritische Bilanz. Innsbruck: Studienverlag, S. 79-98.

Baer, Susanne (2004): Geschlecht und Recht - zur rechtspolitischen Steuerung der Geschlechterverhältnisse. In: Meuser, Michael; Neusüß, Claudia (Hg.): Gender Mainstreaming. Konzepte - Handlungsfelder - Instrumente. Bonn: Bundeszentrale für politische Bildung, S. 71-83.

Bambra, Clare (2007): Defamilisation and welfare state regimes: a cluster analysis. In: International Journal of Social Welfare, Jg. 16, H. 4, S. 326-338.

Bednarz-Braun, Iris (2000): Berufliche Gleichstellungspolitik für Frauen in den USA, Großbritannien und Deutschland. Unterschiede, Gemeinsamkeiten, politische Schlussfolgerungen. Das Forschungsjahr 2000. München: DJI.

Benz, Arthur (2008): Der moderne Staat. München: Oldenbourg.

Berghahn, Sabine (2011): Der Ritt auf der Schnecke. In: gender-politik-online.de, Juli 2011.

Bettio, Francesca; Verashchagina, Alina (2009): Gender segregation in the labour market. Root causes, implications and policy responses in the EU. Brussels: European Commission/EGGE.

Betzelt, Sigrid (2007): „Gender Regimes“: Ein ertragreiches Konzept für die komparative Forschung. Literaturstudie (ZeS-Arbeitspapier Nr. 12/2007). Bremen: Zentrum für Sozialpolitik.

Bieri, Sabine (2006): Developing gender, transforming development: Epistemological shifts in debates on gender and development. In: Muller, Christine; Premchander, 
Smita (Hg.): Gender and sustainable development. Berne: National Center of Competence and Research North-South, S. 57-85.

Biffl, Gudrun (2010): Die ökonomische Situation der Frauen in Österreich. In: Bundesministerin für Frauen und Öffentlichen Dienst im Bundeskanzleramt Österreich (Hg.): Frauenbericht. Bericht betreffend die Situation von Frauen in Österreich im Zeitraum von 1998 bis 2008. Wien: BKA, S. 465-502.

BKA (Bundeskanzleramt) (2010): 8. Gleichbehandlungsbericht des Bundes 2010. Wien.

BKA (Bundeskanzleramt) (2012): Das Personal des Bundes 2012. Wien.

BKA (Bundeskanzleramt) (2013a): Das Personal des Bundes 2013. Wien.

BKA (Bundeskanzleramt) (2013b): Der Einkommensbericht. Ein Praxis-Ratgeber. Wien.

BKA (Bundeskanzleramt) (2014a): 10. Gleichbehandlungsbericht des Bundes 2014. Wien.

BKA (Bundeskanzleramt) (2014b): Einkommensbericht 2014 gemäß § 6a BundesGleichbehandlungsgesetz. Wien.

BMFSFJ (Bundesministerium für Familie, Senioren, Frauen und Jugend) (2009): Der Verdienstunterschied von Frauen und Männern im öffentlichen Bereich und in der Privatwirtschaft. Berlin (http://www.bmfsfj.de/BMFSFJ/Service/Publikationen/ publikationen,did=133762.html; Zugriff: 24.02.2014).

BMFSFJ (Bundesministerium für Familie, Senioren, Frauen und Jugend); BJV (Bundesministerium der Justiz und für Verbraucherschutz) (2014): Gesetz für die gleichberechtigte Teilhabe von Frauen und Männern an Führungspositionen in der Privatwirtschaft und im Öffentlichen Dienst - Leitlinien für das Gesetzgebungsverfahren. Berlin (Stand: 25. März 2014).

BMFSFJ (Bundesministerium für Familie, Senioren, Frauen und Jugend); Kienbaum (2014a): Statistischer Datenreport: Durchführung von vorbereitenden statistischen Analysen und Auswertungen zur Umsetzung des Bundesgleichstellungsgesetzes. Berlin: BMFSFJ.

BMFSFJ (Bundesministerium für Familie, Senioren, Frauen und Jugend); Kienbaum (2014b): Datenreport: Vorbereitende statistische Analysen und Auswertungen zur Umsetzung des Bundesgremienbesetzungsgesetzes. Berlin: BMFSFJ.

BMFSFJ (Bundesministerium für Familie, Senioren, Frauen und Jugend) (2009). Der Verdienstunterschied von Frauen und Männern im öffentlichen Bereich und in der Privatwirtschaft. Ergebnisse des Projekts „Verdienstunterschiede zwischen Männern und Frauen" des BMFSFJ und des statistischen Bundesamtes. Berlin.: BMFSFJ.

BMI (Bundesministerium des Innern) (2011): Der öffentliche Dienst des Bundes. Daten zur Personalstruktur 2011. Berlin (http://www.bmi.bund.de/SharedDocs/ Downloads/DE/Broschueren/2011/personalstruktur.pdf?_blob=publicationFile; Zugriff: 08.01.2014).

Bogner, Alexander; Littig, Beate; Menz, Wolfgang (Hg.) (2005): Das Experteninterview. Theorie, Methode, Anwendung. 2. Aufl. Wiesbaden: VS Verlag für Sozialwissenschaften.

Bogumil, Jörg; Jann, Werner (2009): Verwaltung und Verwaltungswissenschaft in Deutschland. Einführung in die Verwaltungswissenschaft (2. Aufl.). Wiesbaden: VS Verlag für Sozialwissenschaften. 
Bothfeld, Silke (2005): Grenzen des Politischen Lernens, Grenzen des Gender Mainstreaming. Was bewirkt Gender Mainstreaming? Evaluierung durch Policy-Analyse. In: Behning, Ute; Sauer, Birgit (Hg.): Was bewirkt Gender Mainstreaming? Evaluierung durch Policy-Analyse. Frankfurt/M.: Campus, S. 149-173.

Bothfeld, Silke (2008): Under reconstruction: Die Fragmentierung des deutschen Geschlechterregimes durch die neue Familienpolitik (ZeS-Arbeitspapier Nr. 1/ 2008). Bremen: Zentrum für Sozialpolitik.

Bothfeld, Silke; Hübers, Sebastian; Rouault, Sophie (2010): Gleichstellungspolitische Rahmenbedingungen für das betriebliche Handeln. Ein internationaler Vergleich. In: Projektgruppe GiB (Hg.): Geschlechterungleichheiten im Betrieb. Arbeit, Entlohnung und Gleichstellung in der Privatwirtschaft. Berlin: edition sigma, S. 2388.

Bothfeld, Silke; Kuhl, Mara (2008): Gleichstellungspolitik und feministische Politikwissenschaft - eine „unsichtbare Avantgarde“ der Governance-Forschung? In: Schuppert, Gunnar Folke; Zürn, Michael (Hg.): Governance-Forschung. Sonderheft der Politischen Vierteljahresschrift. Wiesbaden: VS Verlag für Sozialwissenschaften, S. 330-351.

Bothfeld, Silke; Rouault. Sophie (2015): Was macht eine effiziente Gleichstellungspolitik aus? Zur Koordination und Ausgestaltung von Instrumenten der beruflichen Gleichstellung. In: WSI-Mitteilungen, H. 1, S. 25-34.

Braunmühl, Claudia von (2001): Gender Mainstreaming Worldwide - Rekonstruktion einer Reise um die Welt. In: Österreichische Zeitschrift für Politikwissenschaft, H. 2, S. 183-201.

Bühler, Elisabeth; Heye, Corinna (2005): Eidgenössische Volkszählung 2000: Fortschritte und Stagnation in der Gleichstellung der Geschlechter 1970-2000. Neuchâtel: Bundesamt für Statistik.

Bundesamt für Landwirtschaft (2010): Stellenbeschrieb: Beauftragte/r für Chancengleichheit. Ms., März 2010.

Bundesamt für Statistik (2013): Pendlermobilität in der Schweiz 2011 (BFS aktuell). Neuchâtel: BFS.

Bundesrat (1998): Botschaft vom 14. Dezember 1998 betreffend das Bundespersonalgesetz (BPG), BB1. 1999 I 1597. Bern.

Bütler, Monika (2007): Arbeiten lohnt sich nicht - ein zweites Kind noch weniger. Zum Einfluss einkommensabhängiger Tarife in der Kinderbetreuung. In: Perspektiven der Wirtschaftspolitik, Jg. 8, H. 1, S. 1-19.

Charles, Maria (2005): Entwicklung der beruflichen Segregation nach Geschlecht und nach Staatsangehörigkeit in der Schweiz. Neuchâtel: BFS.

Conley, Hazel; Page, Margaret (2010): The Gender Equality Duty in Local Government: The Prospects for Integration. In: Industrial Law Journal, Jg. 39, H. 3, S. 321-325.

Conley, Hazel; Page, Margaret (2014): Gender equality in public services. Chasing the dream. London: Routledge.

Dackweiler, Regina-Maria (2005): Die Konstruktion von Geschlechter-Wirklichkeit durch den Wohlfahrtsstaat. In: Henninger, Annette; Ostendorf, Helga (Hg.): Die politische Steuerung des Geschlechterregimes. Beiträge zur Theorie politischer Institutionen. Wiesbaden: VS Verlag für Sozialwissenschaften, S. 75-92.

Demmke, Christoph (2011): Die Reform der öffentlichen Dienste im internationalen Vergleich. In: Koch, Rainer; Conrad, Peter; Lorig, Walter H. (Hg.): New Public 
Service. Öffentlicher Dienst als Motor der Staats- und Verwaltungsmodernisierung (2. Aufl.). Wiesbaden: Gabler, S. 55-83.

Destatis (2012): Finanzen und Steuern. Personal des öffentlichen Dienstes 2011. Fachserie 14, Reihe 6. Wiesbaden: Statistisches Bundesamt.

Deutscher Bundestag (2010a): Zweiter Erfahrungsbericht der Bundesregierung zum Bundesgleichstellungsgesetz (Berichtszeitraum: 1. Juli 2004 bis 30. Juni 2009). Drucksache 17/4307 vom 16.12.2010. Berlin.

Deutscher Bundestag (2010b): Fünfter Gremienbericht der Bundesregierung zum Bundesgremienbesetzungsgesetz (Berichtszeitraum: 30. Juni 2005 bis 30. Juni 2009). Drucksache 17/4308 (neu) vom 16.12.2010. Berlin.

Deutscher Bundestag (2014): Entwurf eines Gesetzes für die gleichberechtigte Teilhabe von Frauen und Männern an Führungspositionen in der Privatwirtschaft und im öffentlichen Dienst. Drucksache 18/3784 vom 20.01.2015. Berlin.

Deutscher Juristinnenbund (2014): Stellungnahme zum Referentenentwurf des Bundesministeriums für Familien, Senioren, Frauen und Jugend (BMFSFJ) und des Bundesministeriums der Justiz und für Verbraucherschutz (BMJV) zum Entwurf eines Gesetzes für die gleichberechtigte Teilhabe von Frauen und Männern an Führungspositionen in der Privatwirtschaft und im öffentlichen Dienst vom 7. Oktober 2014. Berlin: DJB.

DEZA (Direktion für Entwicklung und Zusammenarbeit) (1997): Politik zur Chancenförderung von Frauen in der DEZA. Bern.

Doppel, Lena; Brocza, Judith; Haiszan, Romana; Feutl, Manuela; Kofler, Ulrike; Rapf, Martin; Steiner, Karin; Egger, Andrea (2004): Statusbericht - Auf dem Weg von Telearbeit zu eWork. Zum Stand von Telearbeit und eWork in Österreich vor dem Hintergrund der Entwicklungen in der EU. Wien: Bundesministerium für Wirtschaft und Arbeit.

Dörfler, Sonja; Rille-Pfeiffer, Christiane; Buchegger-Traxler, Anita; Kaindl, Markus; Klepp, Doris; Wernhart, Georg (2009): Evaluierung Elternteilzeit. Die Sichtweisen von Eltern, Unternehmen und ExpertInnen zur Neuregelung der Elternteilzeit (ÖIF-Forschungsbericht). Wien: ÖIF.

EBG (Eidgenössisches Büro für die Gleichstellung von Frau und Mann) (2004): Gender Mainstreaming in der Bundesverwaltung. Leitfaden für den Einbezug der Gleichstellung von Frau und Mann in die tägliche Arbeit der Bundesangestellten. Bern.

EBG (Eidgenössisches Büro für die Gleichstellung von Frau und Mann; Sektion für Chancengleichheit und Globale Gender- und Frauenfragen) (2014): Gleichstellung von Frau und Mann. Aktionsplan der Schweiz. Bilanz 1999-2014. Bern.

EDA (Eidgenössisches Departement für auswärtige Angelegenheiten) (2011): Politik Chancengleichheit 2010-2020. Bern.

Ellgut, Peter; Kohaut, Susanne (2011): Der Staat als Arbeitgeber: Wie unterscheiden sich die Arbeitsbedingungen zwischen öffentlichem Sektor und der Privatwirtschaft. In: Industrielle Beziehungen, Jg. 18, H. 1-2, S. 11-38.

Emery, Yves (2013): Neue Politiken und Prozesse im öffentlichen Personalmanagement. In: Ladner, Andreas (Hg.): Handbuch der öffentlichen Verwaltung in der Schweiz. Zürich: Verlag Neue Zürcher Zeitung, S. 479-497.

Engeli, Isabelle (2011): L'évolution de la promotion de l'égalité entre les femmes et les hommes au sein de l'administration: le cas de l'administration fédérale suisse. In: Politiques et management public, Jg. 28, H. 2, S. 181-200. 
EPA (Eidgenössisches Personalamt) (2002): Bericht an den Bundesrat über die zweite Frauenförderungsperiode in der allgemeinen Bundesverwaltung 1996-1999. Bern.

EPA (Eidgenössisches Personalamt) (2004): Chancengleichheit von Frau und Mann in der Bundesverwaltung. Evaluationsbericht. Bern.

EPA (Eidgenössisches Personalamt) (2009): Förderung der Chancengleichheit von Frau und Mann in der Bundesverwaltung 2004 bis 2007. Evaluationsbericht an den Bundesrat. Bern.

EPA (Eidgenössisches Personalamt) (2010): Personalstrategie Bundesverwaltung 2011-2015. Bern.

EPA (Eidgenössisches Personalamt) (2012): Förderung der Chancengleichheit von Frau und Mann in der Bundesverwaltung 2008-2011. Evaluationsbericht an den Bundesrat. Bern.

EPA (Eidgenössisches Personalamt) (2014): Reporting Personalmanagement 2013. Bern.

EPA-Datensatz (2012): Auszug aus den Personaldaten für die Bundesverwaltung 2002-2011, on file with the author. Bern.

European Commission (2014): Report on Progress on equality between women and men in 2013. Report from the Commission to the European Parliament, the Council, the European Economic and Social Committee and the Committee of the Regions. SWD (2014) 142 final, 14.4.2014. Brüssel: European Commission.

EVD (Eidgenössisches Volkswirtschaftsdepartement) (2004): Leitbild für die Chancengleichheit von Frauen und Männern. Bern.

EVD (Eidgenössisches Volkswirtschaftsdepartement) (2007): Konzept Vereinbarkeit von Familie und Beruf. Bern.

Fraser, Nancy (2000): Die Gleichheit der Geschlechter und das Wohlfahrtssystem. Ein postindustrielles Gedankenexperiment. In: Braun, Kathrin; Fuchs, Gesine; Lemke, Christiane; Toens, Katrin (Hg.): Feministische Perspektiven der Politikwissenschaft. München: Oldenbourg, S. 195-215.

Fuchs, Gesine (2008a): Bericht zur Ist-Situation in der Bundesverwaltung, den Kantonen und Städten bezüglich Mutterschaftsentschädigung und parlamentarischen Vorstössen zu Vaterschaft und Elternschaft (inkl. Adoption). Im Auftrag der Schweizerischen Konferenz der Gleichstellungsbeauftragten. Bern.

Fuchs, Gesine (2008b): Suisse: Droits des femmes - un chemin libéral vers l'égalité. In: Chronique internationale de l'IRES, H. 113, S. 32-42.

Fuchs, Gesine (2009): Women in Switzerland: From Backwardness to Uneven Progress. In: Gelb, Joyce; Palley, Marian Lief (Hg.): Women and Politics around the world. A comparative history and survey (Bd. 2). Santa Barbara, Calif.: ABCCLIO, S. 581-603.

Fuchs, Gesine (2011): Interviewstudie zur Wissenssicherung der institutionalisierten Gleichstellungsarbeit in der Schweiz. Im Auftrag der kantonalen Fachstellen für Gleichstellung BL und BE sowie des EBG. Unveröff. Manuskript.

Fuchs, Gesine (2012): Rechtsmobilisierung gegen Geschlechterdiskriminierung im Erwerbsleben - Erfahrungen aus der Schweiz (Kap. 4.10, Lieferung Juli 2012). In: Berghahn, Sabine; Schultz, Ulrike (Hg.): Rechtshandbuch für Frauen- und Gleichstellungsbeauftragte. Recht von A-Z für Frauen- und Gleichstellungsbeauftragte in der Öffentlichen Verwaltung, in Unternehmen und in Beratungsstellen. Hamburg: Dashöfer, S. 1-13. 
Fuchs, Gesine (2013): Recht als feministische Strategie? Überlegungen anhand von Lohngleichheitskämpfen in der Schweiz. In: Streit - Feministische Rechtszeitschrift, Jg. 31, H. 1, S. 3-13.

Fuchs, Gesine (2015a): Substanzielle Repräsentation im Schweizer Parlament: Zum Agenda Setting beruflicher Gleichstellungspolitik 1996-2011. In: femina politica, Jg. 24, H. 1, S. 73-83.

Fuchs, Gesine (2015b): Gleichstellungspolitik in der Schweiz. Entstehung und Steuerung eines umstrittenen Politikfeldes. Opladen: Verlag Barbara Budrich.

Fuchs, Gesine; Bothfeld, Silke (2011): Gleichstellung in Deutschland im internationalen Vergleich. In: Aus Politik und Zeitgeschichte, Jg. 61, H. 37-38, S. 7-18.

Fuchs, Gesine; Debrunner, Yvonne (2014): Entstehung und Steuerung beruflicher Gleichstellungspolitik: Agenda Setting auf Bundesebene 1996-2011. Zürich: Institut für Politikwissenschaft.

Fuchs, Gesine; Hinterhuber, Eva Maria (2015): Komplexe Wechselbeziehungen Geschlechterpolitik in Osteuropa. In: femina politica, Jg. 24, H. 2, S. 8-27.

Fuchs, Gesine; Konstatzky, Sandra; Liebscher, Doris; Berghahn, Sabine (2009): Rechtsmobilisierung für Lohngleichheit: Der Einfluss rechtlicher und diskursiver Bedingungen in der Schweiz, Deutschland und Österreich im Vergleich. In: Kritische Justiz, Jg. 41, H. 3, S. 253-270.

Funder, Maria (Hg.) (2014): The Gender Cage - Revisited. Handbuch zur Organisations- und Geschlechterforschung. Baden-Baden: Nomos.

Geisberger, Tamara; Glaser Thomas (2014): Geschlechtsspezifische Verdienstunterschiede. Analysen zum „Gender Pay Gap“ auf Basis der Verdienststrukturerhebung 2010. In: Statistische Nachrichten 2014, H. 3, S. 2015-2026.

Geppert, Jochen (2009): Gleichstellungspolitische Programme - Ein Rahmen für die Querschnittspolitik Gleichstellung. In: Gleichstellung in der Praxis, H. 3, S. 1317.

Geppert, Jochen; Lewalter, Sandra (2012): Politikfeld Gleichstellung: Institutionalisierungsschritte und Strategien auf Bundesebene. In: Stiegler, Barbara (Hg.): Erfolgreiche Geschlechterpolitik. Ansprüche - Entwicklungen - Ergebnisse. Berlin: Friedrich-Ebert-Stiftung, S. 5-17

Giauque, David (2013): Die Schweizer Bundesverwaltung im internationalen Vergleich: auf der Suche nach einer Verwaltungstradition. In: Ladner, Andreas (Hg.): Handbuch der öffentlichen Verwaltung in der Schweiz. Zürich: Verlag Neue Zürcher Zeitung, S. 47-60.

Goffman, Erving (1986 [1974]): Frame Analysis. An Essay on the Organization of Experience. Boston: Northeastern University Press.

Göhler, Gerhard (1997): Institution, Macht, Repräsentation: Wofür politische Institutionen stehen und wie sie wirken. Baden-Baden: Nomos.

Göhler, Gerhard (2010): Neue Perspektiven politischer Steuerung. In: Aus Politik und Zeitgeschichte, Jg. 60, H. 2-3, S. 34-40.

Gottschall, Karin (1990): Frauenarbeit und Bürorationalisierung. Zur Entstehung geschlechtsspezifischer Trennungslinien in großbetrieblichen Verwaltungen. Frankfurt/M.: Campus.

Gottschall, Karin (2009): Der Staat und seine Diener: Metamorphosen eines wohlfahrtsstaatlichen Beschäftigungsmodells. In: Obinger, Herbert; Rieger, Elmar (Hg.): Wohlfahrtsstaatlichkeit in entwickelten Demokratien. Frankfurt/M.: Campus, S. 461-492. 
Grunow, Dieter (2009): Bürokratieforschung. In: Kaina, Viktoria; Römmele, Andrea (Hg.): Politische Soziologie. Ein Studienbuch. Wiesbaden: VS Verlag für Sozialwissenschaften, S. 353-383.

Gstrein, Michaela; Mateeva, Liliana; Schönpflug, Karin (2013): Bericht betreffend den Abbau der Benachteiligung von Frauen. Wien: BKA.

Hablützel, Peter (2013): Bürokratie - Management - Governance: Schweizer Verwaltung und Verwaltungsführung im Wandel. In: Ladner, Andreas (Hg.): Handbuch der öffentlichen Verwaltung in der Schweiz. Zürich: Verlag Neue Zürcher Zeitung, S. 93-106.

Hackl, Elsa; Steger-Mauerhofer, Hildegard (2012): Das Bundes-Gleichbehandlungsgesetz in Theorie und Praxis. Die Entwicklung des Frauenanteils in den Führungsebenen der Bundesministerien in den Jahren 1990, 2000 und 2010. In: Journal für Rechtspolitik, Jg. 20, H. 3, S. 226-235.

Hagenah, Evelyn (1996): Neue Instrumente für eine neue Staatsaufgabe: Zur Leistungsfähigkeit prozeduralen Rechts im Umweltschutz. In: Grimm, Dieter; Hagenah, Evelyn (Hg.): Staatsaufgaben. Frankfurt/M.: Suhrkamp Taschenbuch Verlag, S. 487-521.

Häusermann, Silja; Zollinger, Christine (2014): Familienpolitik. In: Knoepfel, Peter; Papadopoulos, Yannis; Sciarini, Pascal; Vatter, Adrian; Häusermann, Silja (Hg.): Handbuch der Schweizer Politik. Zürich: Verlag Neue Zürcher Zeitung, S. 911934.

Haussman, Melissa; Sauer, Birgit (Hg.) (2007): Gendering the state in the age of globalization. Women's movements and state feminism in postindustrial democracies. Lanham: Rowman \& Littlefield.

Henninger, Annette; Ostendorf, Helga (2005): Einleitung: Erträge feministischer Institutionenanalyse. In: Henninger, Annette; Ostendorf, Helga (Hg.): Die politische Steuerung des Geschlechterregime. Beiträge zur Theorie politischer Institutionen. Wiesbaden: VS Verlag für Sozialwissenschaften, S. 9-34.

Holland-Cunz, Barbara (2003): Die alte neue Frauenfrage. Frankfurt/M.: Suhrkamp.

Holst, Elke; Kirsch, Anja (2014): Frauen sind in Vorständen großer Unternehmen in Deutschland noch immer die Ausnahme - moderat steigende Anteile in Aufsichtsräten. In: DIW Wochenbericht, H. 3, S. 19-31.

Hood, Christopher; Margetts, Helen Z. (2007): The tools of government in the digital age (new. ed.). Basingstoke: Palgrave Macmillan.

Howlett, Michael; Ramesh, M.; Perl, Anthony (2009): Studying public policy. Policy cycles \& policy subsystems (3. Aufl.). New York: Oxford University Press.

Huber, Evéline; Bergman, Manfred Max (2013): Zwischen Wunsch und Realität: Ausbildungs- und Berufsverläufe von jungen Frauen. In: Schweizerische Zeitschrift für Bildungswissenschaften, Jg. 35, H. 1, S. 181-199.

Jarosch, Monika (2001): Frauenquoten in Österreich. Grundlagen und Diskussion (Demokratie und Gesellschaft im 21. Jahrhundert, Bd. 2). Innsbruck: Studienverlag.

Jüngling, Christiane; Rastetter, Daniela (2011): Die Implementation von Gleichstellungsmaßnahmen: Optionen, Widerstände und Erfolgsstrategien. In: Krell et al. 2011, S. 25-40.

Kadishi, Bernadette (2001): Schlüsselkompetenzen wirksam erfassen. Personalselektion ohne Diskriminierung. Altstätten: Tobler Verlag. 
Kay, Rosemarie (2011): Gewinn und Auswahl von MitarbeiterInnen. In: Krell et al. 2011, S. 241-260.

Keller, Stefan (2012): Erinnerung an die Beamten. In: Keller, Stefan: 100 Jahre Personalverband des Bundes PVB. Geschichte und Porträts. Hrsg. v. Personalverband des Bundes. Ostermundigen: Personalverband des Bundes, S. 81-84.

Kienbaum (2012): Frauen - Macht - Regierung. Eine Studie über den Anteil der Frauen in Führungspositionen der Bundesregierung 2012. Berlin: Kienbaum Management Consultants $\mathrm{GmbH}$.

Klenner, Christina (2004): Gender - Ein Fremdwort für Betriebsräte? WSI-Mitteilungen, H. 5, S. 277-285.

Klenner, Christina; Brehmer, Wolfram; Plegge, Mareen; Bohulskyy, Yann (2013): Förderung der Vereinbarkeit von Familie und Beruf in Tarifverträgen und Betriebsvereinbarungen in Deutschland - Eine empirische Analyse (WSI-Diskussionspapier 184). Düsseldorf: WSI.

Kling, Gudrun (1999): Die rechtliche Konstruktion des ,weiblichen Beamten“. Frauen im öffentlichen Dienst des Großherzogtums Baden im 19. und frühen 20. Jahrhundert. In: Gerhard, Ute (Hg.): Frauen in der Geschichte des Rechts. München: Beck, S. 600-616.

Klöti, Ulrich; Papadopoulos, Yannis; Sager, Fritz (2014): Regierung. In: Knoepfel, Peter; Papadopoulos, Yannis; Sciarini, Pascal; Vatter, Adrian; Häusermann, Silja (Hg.): Handbuch der Schweizer Politik. Zürich: Verlag Neue Zürcher Zeitung, S. 193-218.

Knoepfel, Peter (2000): Policykiller - Institutionenkiller - ein Triptychon zum Verhältnis zwischen institutionellen und substanziellen öffentlichen Politiken. In: Knoepfel, Peter; Linder, Wolf (Hg.): Verwaltung, Regierung und Verfassung im Wandel. Gedächtnisschrift für Raimund E. Germann. Basel: Helbing \& Lichtenhahn, S. 285-300.

Koch, Angelika (2003): Equal employment policy in Germany. Limited results and prospects for reform. In: Review of Policy Research, Jg. 20, H. 3, S. 443-457.

Koch, Angelika (2007): Teilzeitregelung in Führungspositionen für Beschäftigte mit Kindern. In: Aus Politik und Zeitgeschichte, Jg. 57, H. 7, S. 21-26.

Koch, Angelika (2008): Elternzeit - Teilzeit - Aus(zeit)? Teilzeitrechte in Führungspositionen. In: WSI-Mitteilungen, H. 11+12, S. 612-618.

Kodré, Petra; Müller, Henrike (2003): Shifting Policy Frames: EU Equal Treatment Norms and Domestic Discourses in Germany. In: Liebert, Ulrike (Hg.): Gendering Europeanisation. Brüssel etc.: Verlag Peter Lang, S. 83-116.

Koller, Christophe (2013): Profil des Personals der öffentlichen Hand. In: Ladner, Andreas (Hg.): Handbuch der öffentlichen Verwaltung in der Schweiz. Zürich: Verlag Neue Zürcher Zeitung, S. 499-520.

Kreisky, Eva; Walther, Ingrid (1990): Quantitative und Qualitative Evaluierung des „Förderungsprogramms für Frauen im Bundesdienst“ für den Zeitraum 19811988 (IHS-Forschungsbericht). Wien: IHS

Krell, Gertraude (2011): Diskriminierungs- und Gleichstellungspotenzial von Leistungsbeurteilungen. In: Krell et al. 2011, S. 261-274.

Krell, Gertraude; Ortlieb, Renate; Sieben, Barbara (Hg.) (2011): Chancengleichheit durch Personalpolitik. Gleichstellung von Frauen und Männern in Unternehmen und Verwaltungen. Wiesbaden: Gabler. 
Kremer, Stefanie; Bothfeld, Silke (2012): Reflexive Regulierung im Arbeitsmarkt Königsweg oder Sackgasse? In: Bothfeld, Silke; Bogedan, Claudia; Sesselmeier, Wolfgang (Hg.): Arbeitsmarktpolitik in der Sozialen Marktwirtschaft. Wiesbaden: VS Verlag für Sozialwissenschaften, S. 309-322.

Krings, Franciska; Schär Moser, Marianne; Mouton, Audrey (2013): Sexuelle Belästigung am Arbeitsplatz: Wer belästigt wen, wie und warum? Besseres Verständnis heisst wirksamere Prävention. Bern (http://www.nfp60.ch/SiteCollection Documents/Projekte/nfp60_projekte_krings_zusammenfassung_projektergebnisse_ lang.pdf; Zugriff: 10.09.2014).

Kuhlmann, Sabine; Wollmann, Hellmut (2013): Verwaltung und Verwaltungsreformen in Europa. Einführung in die vergleichende Verwaltungswissenschaft. Wiesbaden: Springer VS.

Lang, Sabine (2007): Gender Governance in Post-Unification Germany: Between Institutionalization, Deregulation and Privatization. In: Outshoorn, Joyce; Kantola, Johanna (Hg.): Changing state feminism. Basingstoke, Hampshire: Palgrave Macmillan, S. 124-143.

Lang, Sabine (2009): Contested Institutionalisation: Women's Policy Agencies and Challenges to Gender Equality in Germany. In: German Politics, Jg. 18, H. 1, S. $55-70$.

Lascoumes, Pierre; Le Galès, Patrick (2007): Introduction: Understanding Public Policy through Its Instruments - From the Nature of Instruments to the Sociology of Public Policy Instrumentation. In: Governance, an international journal of policy and administration, Jg. 20, H. 1, S. 1-21.

Lauth, Hans-Joachim; Pickel, Gert; Pickel, Susanne (2009): Methoden der vergleichenden Politikwissenschaft. Wiesbaden: VS.

Leitner, Andrea (2007): Frauenförderung im Wandel. Gender Mainstreaming in der österreichischen Arbeitsmarktpolitik. Frankfurt/M.: Campus.

Leitner, Andrea; Dibiasi, Anna (2015): Frauenberufe - Männerberufe. Berufliche Segregation in Österreich und Wien (IHS-Forschungsbericht). Wien: IHS.

Leitungsgruppe des NFP 60 Gleichstellung der Geschlechter (2014): Ergebnisse und Impulse. Synthesebericht. Bern.

Lepperhoff, Julia. (2010): Im Schatten der Familienpolitik: Gleichstellungs- und Antidiskriminierungspolitik der Großen Koalition. In: Auth, Diana; Buchholz, Eva; Janczyk, Stefanie (Hg.): Selektive Emanzipation: Analysen zur Gleichstellungs- und Familienpolitik. Opladen: Verlag Barbara Budrich, S. 25-45.

Lewalter, Sandra; Geppert, Jochen; Baer, Susanne (2009): Leitprinzip Gleichstellung? - 10 Jahre Gender Mainstreaming in der deutschen Bundesverwaltung. In: GENDER - Zeitschrift für Geschlecht, Kultur und Gesellschaft, Jg. 1, H. 1, S. 125-139.

Liebig, Brigitte (2003): Leitbilder der Chancengleichheit. Zwischen Werte-, Leistungs- und Verantwortungsorientierung. In: Honegger, Claudia; Liebig, Brigitte; Wecker, Regina (Hg.): Wissen, Gender, Professionalisierung. Historisch-soziologische Studien. Zürich: Chronos, S. 131-148.

Liebig, Brigitte (2005): Zwischen sozialer Gerechtigkeit und Nutzenorientierung: Arbeitskultur und Geschlechtergleichstellung in IT-Betrieben. In: WISO, Jg. 28, H. 2, S. 49-66.

Liebig, Brigitte (2007): „Tacit knowledge“ und Management: Ein wissenssoziologischer Beitrag zur qualitativen Organisationskulturforschung. In: Bohnsack, Ralf; 
Nentwig-Gesemann, Iris; Nohl, Arnd-Michael (Hg.): Die dokumentarische Methode und ihre Forschungspraxis. Grundlagen qualitativer Sozialforschung. Wiesbaden: VS Verlag für Sozialwissenschaften, S. 147-165.

Liebig, Brigitte (2013): Organisationskultur und Geschlechtergleichstellung. Eine Typologie betrieblicher Gleichstellungskulturen. In: Riegraf, Ursula; Müller, Birgit; Wilz, Sylvia M. (Hg.): Geschlecht und Organisation. Wiesbaden: Springer VS, S. 292-31.

Lijphart, Arend (1975): The Comparable Cases Strategy in Comparative Research. In: Comparative Political Studies, Jg. 8, H. 2, S. 158-177.

Linder, Wolf (2005): Schweizerische Demokratie. Zürich: Verlag Neue Zürcher Zeitung.

Lowi, Theodore (1972): Four Systems of Policy, Politics and Choice. In: Public Administration Review, Jg. 32, H. 4, S. 300-310.

Lüthi, Ruth (2014): Parlament. In: Knoepfel, Peter; Papadopoulos, Yannis; Sciarini, Pascal; Vatter, Adrian; Häusermann, Silja (Hg.): Handbuch der Schweizer Politik. Zürich: Verlag Neue Zürcher Zeitung, S. 169-192.

Lutz, Hedwig (2004): Wiedereinstieg und Beschäftigung von Frauen mit Kleinkindern. Ein Vergleich der bisherigen Karenzregelung mit der Übergangsregelung zum Kinderbetreuungsgeld (WIFO Monographien 3/2004). Wien: Wifo.

Lutz, Hedwig; Schratzenstaller, Margit; Leitner, Andrea; Laimer, Andrea (2013): 50\% des Budgets der aktiven Arbeitsmarktpolitik für Frauen. Implementierung, Umsetzung und Wirkung des Genderbudgetziels. Wien: Wifo.

Mägli, Patrick (2013): Zugänglichkeit der Verwaltungsverordnungen (Weisungen) des Bundes. In: LeGes - Gesetzgebung und Evaluation, H. 1, S. 127-140.

Maier, Matthias Leonhard; Nullmeier, Frank (Hg.) (2003): Politik als Lernprozess. Wissenszentrierte Ansätze der Politikanalyse. Opladen: Leske + Budrich.

Mairhuber, Ingrid; Sardadvar, Karin (2012): Unterhaltsrecht und Unterhaltslogik im Steuer- und Sozialrecht sowie in weiteren relevanten Rechtsbereichen (FORBAForschungsbericht 5/2012). Wien: FORBA.

Mayring, Philipp (2010): Qualitative Inhaltsanalyse. Grundlagen und Techniken (11. Aufl.). Weinheim: Beltz.

Mazur, Amy G. (2009): Comparative gender and policy projects in Europe: Current trends in theory, method and research. In: Comparative European Politics, Jg. 7, H. 1, S. 12-36.

Mazur, Amy G. (o.J.): Does Feminist Policy Matter in Post Industrial Democracies? A Proposed Analytical Roadmap (http://www.csbppl.com/wp-content/uploads/ 2013/09/Does-Feminist-Policy-Matter-in-Post-Industrial-Democracies.pdf; Zugriff: 25.03.2014).

McBride, Dorothy E.; Mazur, Amy G. (2010): The politics of state feminism. Innovation in comparative research. Philadelphia: Temple University Press.

Mecking, Sabine (2005): Chefin oder Mauerblümchen? Frauen in der öffentlichen Verwaltung der Bundesverwaltung. In: Geschichte und Gesellschaft, H. 31, S. 465-497.

Medici, Gabriela (2012): Verpflichtung der Kantone, eine wirksame Gleichstellungspolitik zu betreiben. Besprechung des Bundesgerichtsentscheids 1C_549/2010 vom 21. November 2011. In: Jusletter 2. April 2012 (http://www.jusletter.ch; Zugriff: 09.04.2012). 
Ministerium für Generationen, Familie, Frauen und Integration des Landes NordrheinWestfalen (Hg.) (2008): Frauen verändern EUROPA verändert Frauen. Düsseldorf: Eigenverlag.

Mitsching-Viertel, Uta (2012): Teilzeit und Telearbeit als Arbeitszeitmodelle in der Vereinbarkeit von Beruf und Familie. Fluch oder Segen für die Verwaltung? In: BMF (Hg.): Bundesweite Frühjahrstagung der Gleichbehandlungsbeauftragten mit den Frauenbeauftragten - „20 Jahre B-GIBG - Rollenverständnis und Rollenwandel". Wien.

Morel, Nathalie; Palier, Bruno; Palme, Joakim (Hg.) (2012): Towards a social investment welfare state. Ideas, policies and challenges. Bristol: Policy.

Müller, Catherine; Sander, Gudrun (2005): Gleichstellungs-Controlling. Das Handbuch für die Arbeitswelt. Zürich: vdf.

Müller, Franziska (2013): Staatliche Kontroll- und Durchsetzungsinstrumente zur Verwirklichung der Lohngleichheit. Bericht zuhanden des Bundesamtes für Justiz (BJ) und des Eidgenössischen Büros für die Gleichstellung von Frau und Mann (EBG). Hrsg. v. Eidgenössisches Büro für die Gleichstellung von Frau und Mann und Interface - Institut für Politikstudien. Luzern: Interface.

Müller, Ursula; Riegraf Birgit; Wilz, Sylvia M. (Hg.) (2013): Geschlecht und Organisation. Wiesbaden: Springer VS.

Naff, Katherine C.; Kellough, J. Edward (2003): Ensuring Employment Equity: Are Federal Diversity Programs Making a Difference? In: International Journal of Public Administration, Jg. 26, H. 12, S. 1307-1336.

Nentwich, Julia C. (2006): Changing Gender: The Discursive Construction of Equal Opportunities. In: Gender, work and organization, Jg. 13, H. 6, S. 499-521.

Neue Zürcher Zeitung (2008): 400 Deza-Angestellte müssen sich neu bewerben, Nr. 162, S. 8.

NZZ am Sonntag (2008): „Die Entwicklungshilfe hat ein zu starkes Eigenleben entwickelt" (Interview mit Micheline Calmy-Rey), Nr. 23, S. 10.

OECD (Organisation for Economic Co-operation and Development) (2009): Government at a Glance 2009. Paris: OECD.

OECD (Organisation for Economic Co-operation and Development) (2010): Fostering Diversity in the Public Service (http://www.oecd.org/gov/pem/44860884.pdf; Zugriff: 25.06.2013).

OECD (Organisation for Economic Co-operation and Development) (2011a): Government at a Glance 2011. Paris: OECD.

OECD (Organisation for Economic Co-operation and Development) (2011b): Public Servants as Partners for Growth. Paris: OECD.

OECD (Organisation for Economic Co-operation and Development) (2012): OECD Family Data (http://www.oecd.org/els/family/database.htm; Zugriff: 25.06.2013).

OECD (Organisation for Economic Co-operation and Development) (2013a): Government at a glance 2013. Paris: OECD.

OECD (Organisation for Economic Co-operation and Development) (2013b): OECD Employment Database (http://www.oecd.org/employment/onlineoecdemployment database.htm; Zugriff: 25.06.2013).

Oertzen, Christine von (1999a): Teilzeit und die Lust am Zuverdienen. Geschlechterpolitik und gesellschaftlicher Wandel in Westdeutschland 1948-1969. Göttingen: Vandenhoeck \& Ruprecht. 
Oertzen, Christine von (1999b): Women, work and the state: Lobbying for part-time work and practical equality in the West German civil service (1958-1969). In: Torstendahl, Rolf (Hg.): State policy and gender system in the two German States and Sweden (1945-1989). Uppsala: Uppsala universitet, S. 79-104.

Ostendorf, Helga (2011): Gender Knowledge and the „Doing Gender" of Political Institutions. Paper presented at the 2nd European Conference on Politics and Gender, 13-15 January 2011. Budapest.

Painter, Martin; Peters, Guy B. (Hg.) (2010): Tradition and public administration. Basingstoke: Palgrave Macmillan.

Peters, Guy B. (2002): The Politics of Tool Choice. In: Salamon, Lester M. (Hg.): The tools of government. A guide to the new governance. Oxford: Oxford University Press, S. 552-564.

Pfarr, Heide (2001): Ein Gesetz zur Gleichstellung der Geschlechter in der Privatwirtschaft. Düsseldorf: Hans-Böckler-Stiftung.

Pfau-Effinger, Birgit (2005): Wandel der Geschlechterkultur und Geschlechterpolitiken in konservativen Wohlfahrtsstaaten - Deutschland, Österreich und Schweiz. Gender Politik Online (http://www.fu-berlin.de/sites/gpo/index.html; Zugriff: 22.10.2015).

Pimminger, Irene (2012a): Existenzsichernde Beschäftigung von Frauen und Männern. Berlin: Agentur für Gleichstellung im ESF.

Pimminger, Irene (2012b): Armut und Armutsrisiken von Frauen und Männern. Berlin: Agentur für Gleichstellung im ESF.

Preglau, Max (2009): Geschlechterpolitische Aspekte der österreichischen Familienpolitik. In: Appelt, Erna (Hg.): Gleichstellung in Österreich - eine Kritische Bilanz. Innsbruck: Studienverlag, S. 113-132.

Prognos AG (2014): Gesamtevaluation der ehe- und familienbezogenen Maßnahmen und Leistungen in Deutschland. Endbericht. Berlin: Prognos.

Publicus. Schweizer Jahrbuch des öffentlichen Lebens = annuaire suisse de la vie publique $=$ annuario svizzero della vita pubblica. Basel: Verlag/Editions Schwabe.

Pütz. Lasse; Weckes, Marion (2014): Geschlechterquote für mehr Frauen in den Aufsichtsräten - vor allem Anteilseigner sind gefordert. Düsseldorf: Hans-Böckler-Stiftung.

Raasch, Sibylle (1999): Verhandeltes Recht statt materiellem Gleichstellungsgesetz für die Privatwirtschaft? In: Kritische Justiz, H. 2, S. 624-637.

Rechnungshof (2014): Bericht des Rechnungshofes über die durchschnittlichen Einkommen der gesamten Bevölkerung (Allgemeiner Einkommensbericht). Wien.

Reichard, Sven; Schröter, Eckard (2009): Der öffentliche Dienst im Wandel der Zeit: Tradierte Probleme, aktuelle Herausforderungen und künftige Reformperspektiven. In: dms - der moderne staat, H. 1, S. 17-36.

Reidl, Sybille; Schiffbänker, Helene; Gregoritsch, Petra; Kernbeiß, Günter; Städtner, Karin (2013): Karenzväter in Zahlen. Wien: Joanneum Research.

Riesenfelder, Andreas (2013): Wiedereinstiegsmonitoring. Ein Überblick über die Ergebnisse der Kohorten 2006 bis 2010 in Österreich und in der Bundeshauptstadt Wien. Wien: AK Wien.

Riesenfelder, Andreas; Sorger, Claudia; Wetzel, Petra; Willsberger, Barbara (2006): Evaluierung der Einführung des Kinderbetreuungsgeldes. Wien: AK Wien. 
Rille-Pfeiffer, Christiane; Kapella, Olaf (2012): Evaluierungsstudie Kinderbetreuungsgeld. Einkommensabhängige und pauschale Bezugsvariante 12+2 Monate. Forschungsbericht $9 / 2012$. Wien: ÖIF.

Rohlfing, Ingo (2008): Vergleichende Fallanalysen. In: Pickel, Susanne; Pickel, Gert; Lauth, Hans-Joachim; Jahn, Detlef (Hg.): Vergleichende politikwissenschaftliche Methoden.: VS Verlag für Sozialwissenschaften, S. 133-151.

Rose-Möhring, Kristin (2015): Stellungnahme zu Artikel 2 des Entwurfs eines Gesetzes für die gleichberechtigte Teilhabe von Frauen und Männern an Führungspositionen in der Privatwirtschaft und im öffentlichen Dienst (in der Beschlussfassung des Kabinetts vom 11.12.2014) - Neufassung des Bundesgleichstellungsgesetzes (BGleiG). Deutscher Bundestag, Ausschuss für Familie, Senioren, Frauen und Jugend, 11.02.2015.

Rosenberger, Sieglinde (2009): Institutionalisierung durch Europäisierung? Zur Einrichtung von Gleichstellungsinstitutionen zwischen 1995 bis 2008. In: Gleichstellung in Österreich - eine kritische Bilanz. Innsbruck: Studienverlag, S. 57-70.

Rosenberger, Sieglinde; Sauer, Birgit. (Hg.) (2004): Politikwissenschaft und Geschlecht: Konzepte - Verknüpfungen - Perspektiven. Wien: WUV Facultas.

Rowhani, Inge (1991) Frauenarbeit im Sozialressort. In: Angerer, Marie-Luise; Appelt, Erna; Bell, Anni; Rosenberger, Sieglinde; Seidl, Hedwig (Hg.): Auf glattem Parkett. Feministinnen in Institutionen. Wien: Verlag für Gesellschaftskritik, S. $177-204$.

Rudolph, Clarissa (2009): Frauen- und Gleichstellungspolitik: Gesellschaftlicher Wandel durch Institutionen? In: Kurz-Scherf, Ingrid; Lepperhoff, Julia; Scheele, Alexandra (Hg.): Feminismus, Kritik und Intervention. Münster (Westf.): Westfälisches Dampfboot, S. 115-132.

Rudolph, Clarissa; Schirmer, Uta (2004): Gestalten oder verwalten? Kommunale Frauenpolitik zwischen Verrechtlichung, Modernisierung und Frauenbewegung. Wiesbaden: VS Verlag für Sozialwissenschaften.

Sager, Fritz (2009): Die Evaluation institutioneller Politik in der Schweiz. In: Widmer, Thomas; Beywl, Wolfgang; Fabian, Carlo (Hg.): Evaluation. Ein systematisches Handbuch (1. Aufl.). Wiesbaden: VS Verlag für Sozialwissenschaften, S. 361-371.

Sainsbury, Diane (2013): Gender, Care, and Welfare. In: Waylen, Georgina; Celis, Karen; Kantola, Johanna; Weldon, Laurel S. (Hg.): The Oxford handbook of gender and politics. Oxford: Oxford University Press, S. 313-336.

Sancar, Annemarie (2011): Gender Mainstreaming in der EZA: Eine kritische Einschätzung. In: Bieri, Sabin; Sancar-Flückiger, Annemarie Suzanne: Mehr. Wert. Beiträge zur geschlechtergerechten wirtschaftlichen Entwicklung. Hg. v. Caritas Schweiz und Universität Bern. Bern, Luzern: Schweizerische Eidgenossenschaft, Direktion für Entwicklung und Zusammenarbeit DEZA; Universität Bern, Interdisziplinäres Zentrum für Geschlechterforschung; Caritas Schweiz, S. 2-3.

Sauer, Birgit (2009): Gleichstellungspolitik und neoliberaler Staatsumbau. Chancen und Restriktionen. In: Appelt, Erna (Hg.): Gleichstellung in Österreich - eine kritische Bilanz. Innsbruck: Studienverlag, S. 43-56.

Savoie, Donald J. (2011): Administration. In: Badie, Bertrand; Berg-Schlosser, Dirk; Morlino, Leonardo (Hg.): International encyclopedia of political science. Los Angeles: Sage (A Sage reference publication), S. 23-26. 
Scheidegger, Christine (2008): Rahmenbedingungen für die Entstehung und Weiterexistenz von kantonalen Fachstellen für Gleichstellung zwischen 1990 und 2005. Ein Quervergleich. Lizentiatsarbeit Politologie. Bern.

Schiek, Dagmar; Dieball, Heike; Horstkötter, Inge; Seidel, Lore; Vieten, Ulrike M.; Wankel, Sibylle (2002): Frauengleichstellungsgesetze des Bundes und der Länder. Kommentar für die Praxis zu den Gleichstellungsgesetzen, Gleichberechtigungsgesetzen und Frauenfördergesetzen der Länder. Frankfurt/M.: Bund-Verlag.

Schimeta, Julia (2012:. Einsam an der Spitze. Frauen in Führungspositionen im öffentlichen Sektor. Berlin: Friedrich-Ebert-Stiftung.

Schreyögg, Friedel (2012): Kommunale Gleichstellungsarbeit als Reformpolitik. In: Stiegler, Barbara (Hg.): Erfolgreiche Geschlechterpolitik. Ansprüche - Entwicklungen - Ergebnisse. Berlin: Friedrich-Ebert-Stiftung, S. 33-42.

Schubert, Klaus (1991): Politikfeldanalyse - Eine Einführung. Opladen: Leske + Budrich.

Schwegler, Regina; Stern, Susanne; Iten, Rolf (2011): Negative Erwerbsanreize durch Tarife und Steuerabzüge für Familien und schulergänzende Betreuung. Hrsg. v. der Fachstelle für Gleichstellung des Kantons Zürich. Zürich: INFRAS.

Schweikert, Birgit (2002): Alles Gender - oder? Die Implementierung von Gender Mainstreaming auf Bundesebene. In: Bothfeld, Silke; Gronbach, Sigrid; Riedmüller, Barbara (Hg.): Gender Mainstreaming - Ein innovatives Instrument der Gleichstellungspolitik. Zwischenberichte aus der politischen Praxis. Frankfurt/ M.: Campus, S. 83-195.

Schwiter, Karin (2011): Lebensentwürfe. Junge Erwachsene im Spannungsfeld zwischen Individualität und Geschlechternormen. Frankfurt/M.: Campus.

Scott, Joan W. (1986): Gender - a useful category in historical analysis. In: American Historical Review, Jg. 113, H. 5, S. 1053-1075.

Seitz, Werner (2010): Signalisiert die massive Budgetkürzung bei der Zürcher Fachstelle für Gleichstellung vom Dezember 2008 einen Paradigmenwechsel im Umgang mit den Fachstellen? Referat am 40. Treffen der Schweizerischen Konferenz der Gleichstellungsbeauftragten in Bern, 16. März 2010. Bern.

Snow, David A. (2004): Framing Processes, Ideology, and Discursive Fields. In: Snow, David A.; Soule, Sarah A.; Kriesi, Hanspeter (Hg.): The Blackwell Companion to Social Movements. Malden, Oxford, Carlton: Blackwell, S. 380-412.

Stalder, Béatrice; Wägli, Theres (1995): Frauenspezifische Weiterbildung im Betrieb. Eine Untersuchung. Zürich: vdf Hochschulverlag.

Statistik Austria: Bundesstatistiken (http://www.statistik.at/web_de/statistiken/index. html).

Stern, Susanne; Iten, Rolf; Schwab, Stefanie; Felfe, Christina; Lechner, Michael; Thiemann, Petra (2013): Familienergänzende Kinderbetreuung und Gleichstellung. INFRAS/Universität St. Gallen. Zürich (http://www.infras.ch/d/projekte/ gesellschaft.php?id=45\#current; Zugriff: 05.05.2014).

Strachan, Glenda (2011): Equity and diversity in public sector employment. In: Koch, Rainer; Conrad, Peter; Lorig, Walter H. (Hg.): New Public Service. Öffentlicher Dienst als Motor der Staats- und Verwaltungsmodernisierung (2. Aufl.). Wiesbaden: Gabler, S. 345-365. 
Strub, Silvia; Stocker, Désirée (2010): Analyse der Löhne von Frauen und Männern anhand der Lohnstrukturerhebung 2008. Aktuelle Entwicklungen in der Privatwirtschaft und Situation im öffentlichen Sektor des Bundes. Bern: Büro BASS.

Taubner, Johannes; Hirsch, Walter (2013): Der öffentliche Dienst GA 11 (8. Aufl.). Wien (https://www.oeffentlicherdienst.gv.at/vab/seminarprogramm/allgemeine_ ausbildung_und_weiterbildung/SGA_12.pdf?447cx1; Zugriff: 10.01.2014).

Temmes, Markku (2011): Public Employment. In: Badie, Bertrand; Berg-Schlosser, Dirk; Morlino, Leonardo (Hg.): International encyclopedia of political science. Los Angeles: Sage, S. 2157-2159.

Thom, Norbert; Alfes, Kerstin (2007): Strategisches Personalmanagement im öffentlichen Sektor. Anforderung an das Kompetenzprofil von Personalleitern. In: Bräunig, Dietmar; Greiling, Dorothea (Hg.): Stand und Perspektiven der Öffentlichen Betriebswirtschaftslehre II. Berlin: Berliner Wissenschafts-Verlag, S. 567-581.

Töller, Annette E. (2009): Freiwillige Regulierung zwischen Staat und Markt: Der Deutsche Corporate Governance-Kodex (DCGK). In: dms - Der moderne Staat, Jg. 2, H. 2, S. 293-313.

Tondorf, Karin (2010): Lohngleichheit prüfen - aber mit welchen Instrumenten. In: Hohmann-Dennhardt, Christine; Körner, Marita; Zimmer, Reingard (Hg.): Geschlechtergerechtigkeit. Festschrift für Heide Pfarr. Baden-Baden: Nomos, S. 334-345.

Varone, Frédéric (2013): Die Bundesverwaltung. In: Ladner, Andreas (Hg.): Handbuch der öffentlichen Verwaltung in der Schweiz. Zürich: Verlag Neue Zürcher Zeitung, S. 111-127.

Wahl, Angelika von (1999): Gleichstellungsregime. Berufliche Gleichstellung von Frauen in den USA und in der Bundesrepublik Deutschland. Opladen: Leske + Budrich.

Wahl, Angelika von (2005): Liberal, Conservative, Social Democratic, or ... European? The European Union as Equal Employment Regime. In: Social Politics: International Studies in Gender, State and Society, Jg. 12, H. 1, S. 67-95.

Wahl, Angelika von; Hardmeier, Sibylle (2009): Analyzing the Child Rate in Eight OECD Countries: Does reconciliation policy matter. o.O., unveröff. Ms.

Wetterer, Angelika (2002): Strategien rhetorischer Modernisierung - Gender Mainstreaming, Managing Diversity und die Professionalisierung der Gender-Expertinnen. In: Zeitschrift für Frauenforschung und Geschlechterstudie, H. 3, S. 129148.

Wetterer, Angelika (2011): Wer weiß was? Gleichstellungspolitik und Geschlechterwissen wissenssoziologischer Perspektive. In: CEWS (Hg.): Fakten und Fassaden. Gleichstellungspolitiken und Geschlechterwissen in Wissenschaft und Forschung. Bonn, S. 48-63.

Widmer, Thomas; Bothfeld, Silke; Fuchs, Gesine; Leitner, Andrea; Rouault, Sophie; Zollinger, Christine (2014a): Entstehung und Steuerung von schweizerischen Gleichstellungspolitiken zur Erwerbsarbeit Schweiz. Zusammenfassung der Projektergebnisse - Langversion. Zürich (http://www.nfp60.ch/SiteCollection Documents/Projekte/nfp60_projekte_widmer_projektergenisse_lang.pdf; Zugriff: 26.08.2014).

Widmer, Thomas; Fuchs, Gesine; Zollinger, Christine (2014b): Wenn Gleichstellungspolitik zu Ungleichheit führt. In: Neue Zürcher Zeitung, 16. Juli 2014, S. 17. 
Widmer, Thomas; Zollinger, Christine (2014): Vereinbarkeitspolitik als Gefahr für die Gleichstellung und den sozialen Zusammenhalt? In: Soziale Sicherheit CHSS, H. 4, S. 219-223.

Wilz, Sylvia M. (2013): Geschlechterdifferenzierung von und in Organisationen. (EA 2006). In: Müller, Ursula; Riegraf Birgit; Wilz, Sylvia M. (Hg.): Geschlecht und Organisation. Wiesbaden: Springer VS, S. 150-160.

Windhoff-Héritier, Adrienne (1987): Policy-Analyse. Eine Einführung. Frankfurt/M.: Campus.

Wirz, Claudia (2014): Der kleine Unterschied auf dem Lohnzettel, Neue Zürcher Zeitung vom 8. Dezember 2014 (http://www.nzz.ch/wissenschaft/bildung/derkleine-unterschied-auf-dem-lohnzettel-1.18439914; Zugriff: 09.02.2015).

Wrangell, Ute von (2012): Die Gleichstellungsbeauftragte - unverzichtbares Element emanzipatorischer Geschlechterpolitik. In: Stiegler, Barbara (Hg.): Erfolgreiche Geschlechterpolitik. Ansprüche - Entwicklungen - Ergebnisse. Berlin: FriedrichEbert-Stiftung, S. 18-32.

Wright, Tessa; Conley, Hazel (Hg.) (2011): Gower handbook of discrimination at work. Surrey, England: Gower.

Wroblewski, Angela; Leitner, Andrea (2005): „Lernen von den Besten.“ Interdependenzen von Frauenerwerbstätigkeit und Geburtenzahlen im Ländervergleich. Düsseldorf: Hans-Böckler-Stiftung.

Wroblewski, Angela; Leitner, Andrea (2011): Evaluation von excellentia. IHS-Projektbericht. Wien: IHS.

Zollinger, Christine; Fuchs, Gesine (2011): Ad-hoc Recherche zu den Rechtsgrundlagen der Personal- und Gleichstellungspolitik in der Bundesverwaltung. Zürich (unveröff. Ms.). 


\section{Abkürzungen}

$\begin{array}{ll}\text { AMS } & \text { Arbeitsmarktservice } \\ \text { ARGE } & \text { Arbeitskreis für Gleichbehandlungsfragen } \\ \text { BDG } & \text { Beamten-Dienstrechtsgesetz } \\ \text { BG } & \text { Bundesgericht } \\ \text { B-GBK } & \text { Bundes-Gleichbehandlungskommission } \\ \text { B-GlBG } & \text { Bundes-Gleichbehandlungsgesetz } \\ \text { BGBl. } & \text { Bundesgesetzblatt } \\ \text { BGleiG } & \text { Bundesgleichstellungsgesetz } \\ \text { BKA } & \text { Bundeskanzleramt } \\ \text { BLW } & \text { Bundesamt für Landwirtschaft } \\ \text { BMASK } & \text { Bundesministerium für Arbeit, Soziales und Konsumenten- } \\ \text { schutz } \\ \text { BMeiA } & \text { Bundesministerium für europäische und internationale Ange- } \\ \text { BMF } & \text { legenheiten } \\ \text { BMG } & \text { Bundesministerium der Finanzen } \\ \text { BMI } & \text { Bundesministerium des Innern } \\ \text { BMJ } & \text { Bundesministerium der Justiz } \\ \text { BMLFUW } & \text { Bundesministerium für Land- und Forstwirtschaft, Umwelt } \\ \text { BMLVS } & \text { und Wasserwirtschaft } \\ \text { BMUKK } & \text { Bundesministerium für Landesverteidigung und Sport } \\ \text { BMVIT } & \text { Bundesministerium für Verkehr, Innovation und Technologie } \\ \text { BMWF } & \text { Bundesministerium für Wissenschaft und Forschung } \\ \text { BMWFJ } & \text { Bundesministerium für Wirtschaft, Familie und Jugend } \\ \text { BPG } & \text { Bundespersonalgesetz } \\ \text { BPV } & \text { Bundespersonalverordnung } \\ \text { BV } & \text { Bundesverfassung } \\ \text { CEDAW } & \text { Convention on the Elimination of All Forms of Discrimina- } \\ \text { tion against Women - Frauenrechtskonvention } \\ \text { DEZA } & \text { Direktion für Entwicklung und Zusammenarbeit } \\ \text { EBG } & \text { Eidgenössisches Büro für die Gleichstellung von Frau und } \\ & \text { Mann } \\ & \end{array}$


EDA Eidgenössisches Departement für auswärtige Angelegenheiten

EDI Eidgenössisches Departement des Inneren

EFD Eidgenössisches Finanzdepartement

EFV Eidgenössische Finanzverwaltung

EJPD Eidgenössisches Justiz- und Polizeidepartement

EPA Eidgenössisches Personalamt

EVD Eidgenössisches Volkswirtschaftsdepartement (bis 2012)

GBA Gleichstellungsbeauftragte

GlG Gleichstellungsgesetz

GM Gender Mainstreaming

HRK Human-Resource-Konferenz

idgF in der geltenden Fassung

ILO International Labour Organization

IMAG GlB Interministerielle Arbeitsgruppe für Gleichbehandlungsfragen

m.w.N. mit weiteren Nennungen

NPM New Public Management

SECO Staatssekretariat für Wirtschaft

TVöD Tarifvertrag für den öffentlichen Dienst

UVEK Eidgenössisches Departement für Umwelt, Verkehr, Energie und Kommunikation

VBPV Verordnung des EFD zur Bundespersonalverordnung

VBS Eidgenössisches Departement für Verteidigung, Bevölkerungsschutz und Sport

VPOD Verband des Personals der öffentlichen Dienste

WBF Departement für Wirtschaft, Bildung und Forschung ( $a b$ 2013) 


\section{Neue Reihe: Politik und Geschlecht - kompakt}

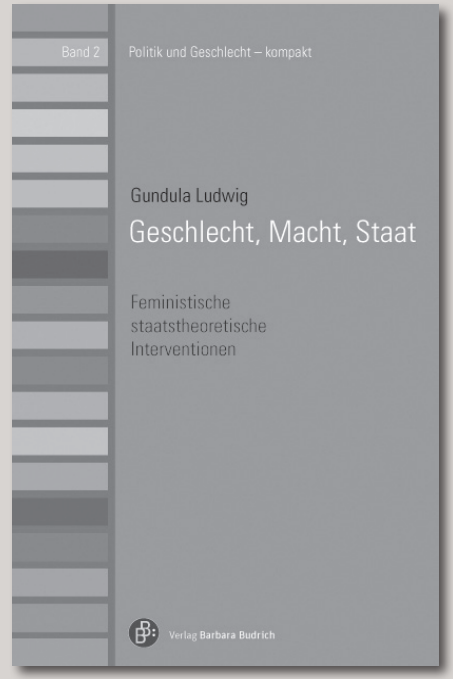

Ziel feministischer Staatstheorie ist es, die Vergeschlechtlichung des Staates sichtbar zu machen. Wie trägt der moderne westliche Staat dazu bei, geschlechtliche Ausbeutungs-, Gewalt- und Ungleichheitsverhältnisse zu ermöglichen und zu legitimieren?

Die Autorin stellt dazu frühe Ansätze feministischer Staatstheorie ebenso vor wie neuere queer-feministische und intersektionale Konzepte.
Gundula Ludwig

Geschlecht, Macht, Staat

Feministische staatstheoretische Interventionen

Politik und Geschlecht kompakt, Band 2

2015. 161 Seiten. Kart. $14,90 €(D), 15,40 €(A)$ ISBN 978-3-8474-0152-0
Jetzt in Ihrer Buchhandlung bestellen oder direkt bei:

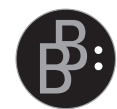

Verlag Barbara Budrich Barbara Budrich Publishers Stauffenbergstr. 7 51379 Leverkusen-Opladen

Tel +49 (0)2171.344.594 Fax +49 (0)2171.344.693 info@budrich.de

\section{www.budrich-verlag.de}




\section{Unsere Fachzeitschriften auf www.budrich-journals.de}
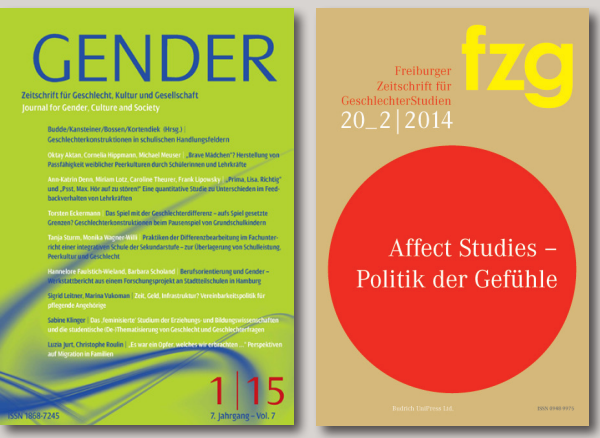

\section{FEMINA POLITICA}

0212014 23. Jg.

Digitalisierung zwischen Utopie und Kontrolle

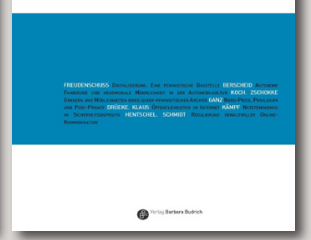

- Einzelbeiträge im Download

- Print + Online Abonnements

- Online-Freischaltung über IP

- mit open access-Bereich

Als AbonnentIn mit einem Kombi-Abo Print + Online bekommen Sie weiterhin Ihr Heft wie gewohnt bequem nach Hause geliefert und Sie haben zusätzlich Zugriff auf das gesamte Online-Archiv.

Verlag Barbara Budrich Barbara Budrich Publishers Stauffenbergstr. 7 D-51379 LeverkusenOpladen

Tel +49(0)2171.344.594 Fax $+49(0) 2171.344 .693$ info@budrich-journals.de

Fragen Sie uns!

www.budrich-journals.de 